\title{
Experimental progress in positronium laser physics
}

\author{
David B. Cassidy ${ }^{\mathrm{a}}$ \\ Department of Physics and Astronomy, University College London, Gower Street, London WC1E 6BT, UK
}

\author{
Received 23 November 2017 / Received in final form 29 December 2017 \\ Published online 27 March 2018 \\ (C) The Author(s) 2018. This article is published with open access at Springerlink.com
}

\begin{abstract}
The field of experimental positronium physics has advanced significantly in the last few decades, with new areas of research driven by the development of techniques for trapping and manipulating positrons using Surko-type buffer gas traps. Large numbers of positrons (typically $\geq 10^{6}$ ) accumulated in such a device may be ejected all at once, so as to generate an intense pulse. Standard bunching techniques can produce pulses with ns (mm) temporal (spatial) beam profiles. These pulses can be converted into a dilute Ps gas in vacuum with densities on the order of $10^{7} \mathrm{~cm}^{-3}$ which can be probed by standard ns pulsed laser systems. This allows for the efficient production of excited Ps states, including long-lived Rydberg states, which in turn facilitates numerous experimental programs, such as precision optical and microwave spectroscopy of Ps, the application of Stark deceleration methods to guide, decelerate and focus Rydberg Ps beams, and studies of the interactions of such beams with other atomic and molecular species. These methods are also applicable to antihydrogen production and spectroscopic studies of energy levels and resonances in positronium ions and molecules. A summary of recent progress in this area will be given, with the objective of providing an overview of the field as it currently exists, and a brief discussion of some future directions.
\end{abstract}

\section{Introduction}

The modern (cf. [1]) concept of antimatter was first revealed in the form of anti-electrons (positrons), predicted to exist by the relativistic quantum theory of Dirac [2-5]. These particles were soon observed experimentally in cloud chamber experiments by Anderson [6,7], and then also by Blackett and Ochlialini [8]. Almost immediately Mohorovičić suggested that a positron and an electron could form a hydrogen-like bound state (which he called "electrum"), evidence of which might be found in astrophysical observations of spectral recombination lines [9]. This suggestion was either unappreciated, or unknown (opinions vary $[10,11]$ ), but in any case was not mentioned in several later works that independently predict the existence of what we now call positronium, and describe in more detail some of its properties. The first of these is the Ph.D. thesis of Pirenne [12,13]. As mentioned by Beck in 1946 [14], Pirenne performed calculations of Ps decay rates in Paris in 1942, but this work was not widely known at the time because of war in Europe.

Two more independent and simultaneous predictions of the existence of Ps were made in the US by Ruark [15] and Wheeler [16]. Wheeler's paper, which also describes Ps ions and molecules (polyleptons), contains the following footnote:

"On October 5, four days after the present paper was submitted to the New York Academy of Sciences, the

\footnotetext{
${ }^{\mathrm{a}}$ e-mail: d.cassidy@ucl.ac.uk
}

author learned from Professor Arthur Ruark that he had previously envisaged the existence of the particular entity composed of one electron and one positron. Dr. Ruark has discussed the optical spectrum and the life time of this two-particle system in a note dated September 23, 1945, which he intends to submit for publication to the physical review in the form of a "Letter to the Editor". A reference to unpublished work by L. Landau on the properties of the bi-electron has been made by Alichanian, A. and T. Asatiani. 1945. J. Phys. USSR. 9: 56."

Thus, one could say that a prediction of the basic concept of an electron-positron bound state can be independently attributed to at least five different people (probably more), starting with Mohorovičić. The experimental realization of Ps, however, belongs entirely to Martin Deutsch. In a series of remarkable experiments beginning in 1951 evidence for Ps production was obtained by observing changes in annihilation lifetimes due to interactions with various gases [17]. This result was confirmed shortly thereafter by Pond [18]. At the time there were several three-photon decay rate calculations that did not agree. The measurements were able to resolve the conflict [19], proving that a calculation by Ore and Powell [20] which found a lifetime of $1.4 \times 10^{-7} \mathrm{~s}$ was indeed correct. As pointed out by Deutsch, this was one of the first instances in which "experimental verification of a theoretical result has been possible for a third-order radiation process" [19]. The next experiment in this remarkable series was a measurement of the hyperfine splitting, observed via the magnetic field dependence of singlet-triplet mixing [21]. 
This result, subsequently verified by Pond and Dicke [22], was obtained by observing the fraction of two and threephoton decays in different magnetic fields. Realizing the limitations of this method, a more accurate technique was developed [23-25] which remains the standard method for precision hyperfine interval measurements. Deutsch later went on to perform positron annihilation in flight measurements [26] which also became antecedents of modern day measurements (e.g., $[27,28]$ ).

The work of Deutsch and colleagues did not immediately provoke widespread experimental Ps research, although in the following decades some measurements were conducted, including studies of magnetic quenching [29-31] and electric field effects on Ps in gases [32], (unsuccessful) searches for Ps Lyman alpha lines [33], measurements of Ps formation [34] and diffusion [35] in solid materials, powders [36], and liquids [37], positron polarization measurements [38], symmetry tests [39] and more refined hyperfine interval measurements [40], as well as Ps decay rate measurements [41]. All of this work utilized positrons emitted from radioactive sources and captured in the material in which Ps formation occurred. A significant breakthrough was the development of slow positron beams following positron moderation; creating an energy tunable slow positron beam had been a focus of study for some time [42], with some success [43], but the first instance in which a slow positron beam was used to perform a measurement was in the determination of positron-helium scattering cross sections at University College London (UCL) [44]. In this work a "smoked MgO" moderator was employed, producing around 2 positrons per second. At around the same time a beam of 0.5 slow positrons per second was produced using $\mathrm{Au}$ on mica as a moderator [45]. However, moderation efficiencies were soon improved by orders of magnitude [46], and useful slow positron beams became an experimental reality [47].

Experiments conducted using slow positron beams led to an improved understanding of positron-solid interactions [48], which in turn made it easier to generate higher intensity slow positron beams (e.g., [49-51]) as well as more efficient ways to generate Ps atoms (e.g., [47,52]). In 1986 the solid neon moderator was introduced [53], and this methodology still underpins the state-of-the-art in slow positron beam production using radioactive isotopes today [54-61]. The availability of positron beams has facilitated a great deal of work on experimental atomic positron and Ps physics [62-65], and positron-gas scattering measurements have informed the development of positron buffer-gas traps [66-69].

Thus, most of the essential technical ingredients necessary to conduct experimental Ps research have been available for over 20 years, and many advances have been made in this time, but not with regard to laser spectroscopy. There are several reasons for this: one is that researchers working with positrons have to put considerable effort into beam production, and thus including complicated optical systems increases the scale of the work in a significant way. Collaborative efforts can overcome this problem, assuming researchers with the right skills can overlap. As discussed in Section 3, this has happened occasionally, but such circumstances are rare, and relatively little work on Ps laser spectroscopy was done for decades as a result. The development of positron traps has changed this situation since it is now much easier to generate intense pulsed positron beams in smaller-scale laboratories: at one point Surko traps could even be purchased commercially, although this is no longer the case. Pulsed sources of Ps atoms, ions, and even molecules are ideally suited to laser spectroscopy (as discussed in Sects. 3 and 4), and hence many of the impediments to such work have been removed.

The purpose of this colloquium is to provide a complete overview of the experimental research conducted in this burgeoning field to date. This might appear to be a daunting task but, as is evident from Table 1, most of the this work was been done in the last 8 years. Some excellent reviews of Ps physics are already available, particularly those by Rich [70] and Berko and Pendleton [71]. These reviews focus mainly on fundamental Ps physics and QED tests, and have served as introductions to Ps physics for generations of researchers. However, they are both now more than 35 years old (older than most PhD students). Their continuing utility is in part a testament to the excellence of these articles, but they both predate all Ps laser experiments. Some recent publications cover various areas of Ps laser spectroscopy, (e.g., [72-76]), and Nagashima has written a thorough review on experiments with Ps ions, including photodetachment and optical excitation of shape resonances [77].

Of course, much work has been done in other (nonoptical) areas of experimental positron and positronium research (e.g., [78-80]). Some examples include new measurements of the Ps ground state hyperfine interval that may solve the long-standing discrepancy between theory and experiment [81], the resolution of the (perhaps related) positronium "lifetime puzzle" [82,83], advances in positron scattering from atoms and molecules [84-86], antihydrogen research [87-90], positron beam [91-93] and trap $[66,69]$ development, materials science $[65,94]$, and surface physics [95] (including positron diffraction [96,97] and positron induced Auger emission [98,99]). These areas, and others, will not be discussed here.

\section{Properties of positronium}

The intrinsic properties of Ps atoms (that is, atomic structure and decay modes) are determined by the electromagnetic interaction: the Coulomb force binding a leptonic particle-antiparticle pair and the selection rules governing atomic transitions and annihilation processes give rise to a well defined system that is very well understood theoretically. The extrinsic properties of Ps atoms (e.g., Ps formation efficiencies, velocities and angular distributions or field-induced perturbations) are largely the result of the methods used to create Ps atoms, or the environment in which they are produced. Ps formation generally occurs via energetic interactions on the scale of the Ps binding energy $(6.8 \mathrm{eV})$, but even when Ps atoms can be produced with thermal energies they still have speeds on the order of $100 \mathrm{~km} \mathrm{~s}^{-1}$. In order to conduct experiments with Ps it is usually necessary to develop 
Table 1. Time line of optical excitation experiments involving Ps atoms, ions an molecules. This list includes all instances of Ps excitation with laser light to date. Some of the indicated transitions refer only the excitation pathway, since external fields often preclude the production of pure $\ell$ states. Positron sources used in are radioactive isotopes and gas moderation/Ps production (RI/gas), mono-energetic beams based on radioactive isotopes (RI), magnetic bottle (MB) traps, linac (L) or microtron (M) accelerator based beams, or source-based Surko traps (ST). The light sources used are lamps $(\mathrm{La})$, or pulsed $(\mathrm{P})$ or continuous wave $(\mathrm{CW})$ lasers.

\begin{tabular}{|c|c|c|c|c|c|}
\hline Transition & $e^{+}$source & Light & Comments & Year & Ref. \\
\hline $1^{3} \mathrm{~S}_{1} \rightarrow 2{ }^{3} \mathrm{P}_{\mathrm{J}}$ & $\mathrm{RI} /$ gas & La & No signal observed & 1954 & [100] \\
\hline $1{ }^{3} \mathrm{~S}_{1} \rightarrow 2{ }^{3} \mathrm{P}_{\mathrm{J}}$ & $\mathrm{RI} /$ gas & La & Signal not statistically significant & 1974 & [101] \\
\hline $1^{3} \mathrm{~S}_{1} \rightarrow 2{ }^{3} \mathrm{~S}_{1}$ & $\mathrm{MB}$ & $\mathrm{P}$ & First unambiguous Ps excitation signal & 1982 & {$[102]$} \\
\hline $1{ }^{3} \mathrm{~S}_{1} \rightarrow 2{ }^{3} \mathrm{~S}_{1}$ & MB & $\mathrm{P}$ & Precision measurement (12 ppb) & 1984 & [103] \\
\hline $1{ }^{3} \mathrm{~S}_{1} \rightarrow 2{ }^{3} \mathrm{P}_{\mathrm{J}}$ & $\mathrm{L}$ & $\mathrm{P}$ & Ps Lyman- $\alpha$ saturation & 1990 & [104] \\
\hline $1^{3} \mathrm{~S} \rightarrow 2{ }^{3} \mathrm{P} \rightarrow n^{3} \mathrm{~S} / n^{3} \mathrm{D}$ & $\mathrm{L}$ & $\mathrm{P}$ & First Rydberg Ps & 1990 & [105] \\
\hline $1^{3} \mathrm{~S}_{1} \rightarrow 2{ }^{3} \mathrm{~S}_{1} \rightarrow e^{-}+e^{+}$ & M & $\mathrm{P}$ & Photoexcitation and photoionization & 1991 & {$[106]$} \\
\hline $1{ }^{3} \mathrm{~S}_{1} \rightarrow 2{ }^{3} \mathrm{~S}_{1}$ & M & $\mathrm{CW}$ & Precision measurement (2.6 ppb) & 1993 & {$[107,108]$} \\
\hline $1{ }^{3} \mathrm{~S}_{1} \rightarrow 2{ }^{3} \mathrm{P}_{\mathrm{J}}$ & ST & $\mathrm{P}$ & Ps Doppler spectroscopy & 2010 & {$[109,110]$} \\
\hline $1{ }^{3} \mathrm{~S}_{1} \rightarrow 2{ }^{3} \mathrm{P}_{\mathrm{J}}$ & ST & $\mathrm{P}$ & PsX formation on semiconductors & 2011 & [111-114] \\
\hline $1^{3} \mathrm{~S}_{1} \rightarrow 2{ }^{3} \mathrm{P}_{\mathrm{J}}$ & ST & $\mathrm{P}$ & Ps cavity shift and narrowing & 2011 & [115] \\
\hline $\mathrm{Ps}^{-} \rightarrow \mathrm{Ps}+e^{-}$ & $\mathrm{L}$ & $\mathrm{P}$ & Photodetatchment of $\mathrm{Ps}^{-}$ions & 2011 & [116] \\
\hline $1{ }^{3} \mathrm{~S}_{1} \rightarrow 2{ }^{3} \mathrm{P}_{\mathrm{J}}$ & ST & $\mathrm{P}$ & Excited Ps in Paschen Back regime & 2011 & {$[117]$} \\
\hline $1^{3} \mathrm{~S} \rightarrow 2^{3} \mathrm{P} \rightarrow n^{3} \mathrm{~S} / n^{3} \mathrm{D}$ & ST & $\mathrm{P}$ & Efficient Rydberg Ps production & 2012 & {$[118]$} \\
\hline $\mathrm{Ps}_{2} \rightarrow \mathrm{Ps}+e^{-}+e^{+}$ & ST & $\mathrm{P}$ & Molecular Ps spectroscopy & 2012 & [119] \\
\hline $\mathrm{Ps}^{-} \rightarrow \mathrm{Ps}+e^{-}$ & $\mathrm{L}$ & $\mathrm{P}$ & Energy tunable Ps beam & 2012 & [120] \\
\hline $1{ }^{3} \mathrm{~S}_{1} \rightarrow 2{ }^{3} \mathrm{P}^{\prime} \rightarrow 1{ }^{1} \mathrm{~S}_{0}$ & ST & $\mathrm{P}$ & Saturated absorption spectroscopy & 2012 & [121] \\
\hline $1{ }^{3} \mathrm{~S} \rightarrow 2{ }^{3} \mathrm{P} \rightarrow n^{3} \mathrm{~S} / n^{3} \mathrm{D}$ & ST & $\mathrm{P}$ & Doppler corrected Balmer spectroscopy & 2014 & [122] \\
\hline $1{ }^{3} \mathrm{~S}_{1} \rightarrow 2{ }^{3} \mathrm{~S}_{1}$ & RI & $\mathrm{CW}$ & Annihilation of $2{ }^{3} \mathrm{~S}_{1}$ states & 2015 & [123] \\
\hline $1{ }^{3} \mathrm{~S} \rightarrow 2{ }^{3} \mathrm{P} \rightarrow n^{3} \mathrm{~S} / n^{3} \mathrm{D}$ & ST & $\mathrm{P}$ & High-precision Rydberg TOF & 2015 & [124] \\
\hline $1^{3} \mathrm{~S} \rightarrow 2{ }^{3} \mathrm{P} \rightarrow n^{3} \mathrm{~S} / n^{3} \mathrm{D}$ & ST & $\mathrm{P}$ & Selective production of Ps stark-states & 2015 & [125] \\
\hline $1{ }^{3} \mathrm{~S}_{1} \rightarrow 2{ }^{3} \mathrm{P}_{\mathrm{J}}$ & ST & $\mathrm{P}$ & Laser enhanced Ps TOF & 2015 & [126] \\
\hline $1{ }^{3} \mathrm{~S}_{1} \rightarrow 2{ }^{3} \mathrm{P}_{\mathrm{J}}$ & ST & $\mathrm{P}$ & Ps Doppler spectroscopy & 2015 & {$[127]$} \\
\hline $1{ }^{3} \mathrm{~S}_{1} \rightarrow 2{ }^{3} \mathrm{P}_{\mathrm{J}}$ & ST & $\mathrm{P}$ & Ps cooling in transmission targets & 2015 & [128] \\
\hline $1{ }^{3} \mathrm{~S}_{1} \rightarrow 2{ }^{3} \mathrm{P}^{\prime} \rightarrow 1{ }^{1} \mathrm{~S}_{0}$ & ST & $\mathrm{P}$ & Stark and Zeeman mixing of $n=2$ Ps & 2015 & {$[129,130]$} \\
\hline $1^{3} \mathrm{~S}_{1} \rightarrow 2{ }^{3} \mathrm{P}_{\mathrm{J}}$ & ST & $\mathrm{P}$ & Ps production in cryogenic environments & 2016 & [131] \\
\hline $1{ }^{3} \mathrm{~S} \rightarrow 2{ }^{3} \mathrm{P} \rightarrow 30{ }^{3} \mathrm{~S} / 30{ }^{3} \mathrm{D}$ & ST & $\mathrm{P}$ & Ps Stark-states & 2016 & [132] \\
\hline $\mathrm{Ps}^{-} \rightarrow \mathrm{Ps}+e^{-}$ & $\mathrm{L}$ & $\mathrm{P}$ & $\mathrm{Ps}^{-}$ion shape resonance & 2016 & [133] \\
\hline $1^{3} \mathrm{~S} \rightarrow 2{ }^{3} \mathrm{P} \rightarrow n^{3} \mathrm{~S} / n^{3} \mathrm{D}$ & ST & $\mathrm{P}$ & Measurement of fluorescence lifetimes & 2016 & [134] \\
\hline $1^{3} \mathrm{~S} \rightarrow 3^{3} \mathrm{P} \rightarrow n^{3} \mathrm{~S} / n^{3} \mathrm{D}$ & ST & $\mathrm{P}$ & $n=3 /$ Rydberg excitation $(n=15,16)$ & 2016 & [135] \\
\hline $1^{3} \mathrm{~S} \rightarrow 2{ }^{3} \mathrm{P} \rightarrow 10^{3} \mathrm{~S} / 10^{3} \mathrm{D}$ & ST & $\mathrm{P}$ & Electrostatic guiding of Ps & 2016 & [136] \\
\hline $1^{3} \mathrm{~S} \rightarrow 2^{3} \mathrm{P} \rightarrow 30^{3} \mathrm{~S} / 30^{3} \mathrm{D}$ & ST & $\mathrm{P}$ & Angle resolved Ps spectroscopy & 2016 & {$[137]$} \\
\hline $1^{3} \mathrm{~S} \rightarrow 2^{3} \mathrm{P} \rightarrow n^{3} \mathrm{~S} / n^{3} \mathrm{D}$ & ST & $\mathrm{P}$ & Rydberg Ps MCP detection & 2016 & [138] \\
\hline $1{ }^{3} \mathrm{~S} \rightarrow 2{ }^{3} \mathrm{~S}_{1}^{\prime} \rightarrow 2{ }^{3} \mathrm{~S}_{1}$ & ST & $\mathrm{P}$ & 1-photon production of $2{ }^{3} \mathrm{~S}_{1}$ atoms & 2017 & [139] \\
\hline $1{ }^{3} \mathrm{~S} \rightarrow 2{ }^{3} \mathrm{P} \rightarrow 14{ }^{3} \mathrm{~S} / 14^{3} \mathrm{D}$ & ST & $\mathrm{P}$ & Curved guide Ps velocity selection & 2017 & [140] \\
\hline $1{ }^{3} \mathrm{~S} \rightarrow 2{ }^{3} \mathrm{P} \rightarrow 32{ }^{3} \mathrm{~S} / 32{ }^{3} \mathrm{D}$ & ST & $\mathrm{P}$ & Electrostatic Ps mirror & 2017 & [141] \\
\hline
\end{tabular}

strategies to deal with both the intrinsic and extrinsic Ps properties.

\subsection{Intrinsic properties of positronium}

Why is positronium an interesting system to study? This is a question that demands an answer because experiments that include Ps are generally technically challenging, and one should therefore have a good reason to expend the necessary effort (and funds). There are in fact many reasons why Ps is worth studying, related to both its intrinsic properties as well as how Ps atoms interact with other systems. The formation of an atomic system from a particle-antiparticle pair can result in some unusual features:

- The constituents of Ps are structureless point particles: this means that only electromagnetic forces have to be considered. In principle weak interactions apply, but at a level that is very far away from any experiment currently conceivable $[142,143]$. Therefore Ps is extremely well described by (boundstate) quantum electrodynamics (QED) [144,145], and hence can be used to test the theory. Furthermore, since it lacks any hadronic component (in contrast to hydrogen, in which the proton structure affects the energy levels), it may be possible to use 
Ps to measure the Rydberg constant to sufficiently high accuracy to address the proton radius puzzle (Sect. 5.2).

- The electron and positron have the same mass: this means that recoil effects [146], which in hydrogen would be suppressed by a factor of $M_{p} / m_{e}=1836$, are maximal in Ps (where the mass ratio is 1) and have to be properly taken into account (e.g., [147]). It also means that what we would call hyperfine effects in hydrogen, arising from nuclear spin interactions, are much stronger in Ps. The positron magnetic moment is much larger than that of the heavy, quark-filled, proton, and hence spin-spin interactions contribute more to Ps energy levels than the corresponding interactions in hydrogen [148].

- The constituents are a particle-antiparticle pair: this means that annihilation channels (both real and virtual) contribute significantly to the energy level structure, even at the lowest order [148]. It also means that Ps has no net additive quantum numbers, and is therefore an eigenstate of the charge conjugation operator $\mathrm{C}$, and of $\mathrm{CP}$, the combined operation of $\mathrm{C}$ and the parity operator $\mathrm{P}$. Thus, observations of Ps decay properties can be used to test fundamental symmetries [149].

Much progress has been made in performing QED calculations of Ps properties, and currently all QED corrections to Ps energy levels are known up to order $m \alpha^{6}[150$ 152], with many higher order terms also calculated (e.g., [153-155]). The theoretical precision of these calculations is much higher than that of any measurements. For example, the most precise hyperfine interval measurements are at the $\mathrm{MHz}$ level, whereas calculations are at the $\mathrm{kHz}$ level (see Sect. 5.2).

Thus, more accurate experiments are required to test theory [156]. New experiments may also contribute in other areas; for example, measuring the Rydberg constant in the (leptonic) Ps system, may provide some input to the "proton radius puzzle" as measured in muonic hydrogen [157-159], since there will be no hadronic complications. Unlike 1S-2P transitions, the natural linewidths of transitions between Rydberg states is not limited by annihilation lifetimes, hence, it should be possible to approach the accuracies obtained in experiments using hydrogen or other atoms, which are determined by other factors, such as interaction times [160]. In principle Ps Rydberg constant measurements could become comparable with those conducted with Rydberg hydrogen (e.g., [161,162]).

Several measurements of Ps decay rates have also been undertaken. For many years an apparent anomaly between measured triplet Ps decay rates and calculations (e.g., [163]) seemed to indicate that there might be some new physics accessible by Ps studies. Subsequent experiments have shown that the disagreement was caused by a contribution from non-thermalized Ps [82,83]. A smaller but persistent disagreement between theory and measurements of the ground state hyperfine interval may well be similarly resolved [81].

In addition to testing QED and general symmetry theories, Ps experiments may also help to resolve some of the most important unanswered questions in physics, such as the apparent lack of antimatter in the Universe $[164,165]$. The standard model of particle physics is entirely symmetric in terms of matter and antimatter, and even though we know this model is in some way incomplete, there is still no convincing explanation for how the universe came to be dominated by matter (e.g., [166]). The possibility that $\mathrm{CP}$ violation could be related to this problem was pointed out many years ago by Sakharov [167]. Moreover, there is renewed interest in CP violation in the lepton sector following the discovery that neutrinos are not massless [168].

Similarly, gravitational interactions of antimatter (see Sect. 5.5) which may be connected to CP violation [169], is an area in which any unexpected observation could have far-reaching implications. There are in fact many arguments against the possibility that matter and antimatter could have different gravitational interactions [170], but in the absence of direct measurements there is always room for theoretical creativity. A direct measurement really means something that is as model-independent as possible, such as a simple free-fall or interferometric measurement involving an antiparticle, anti-atom or a composite system containing antimatter (such as Ps or muonium). Performing such an experiment could provide useful information, even if it acts simply to confirm that there is no gravitational difference between matter and antimatter, since it would put restrictions on the types of new forces, particles, or fields theorists could invent in order to solve the mysteries of dark matter, dark energy, the mass hierarchy, the presence of $\mathrm{CP}$ violation in the standard model, and the matter-antimatter asymmetry of the universe.

\subsubsection{The atomic structure of Ps}

In order to facilitate later discussions it is useful to briefly consider the atomic structure of Ps. I use the convention [71] in which Ps states are denoted using the spectroscopic notation $n^{2 S+1} \ell_{J}$, where $n$ is the principal quantum number, $S$ is the total spin quantum number, $\ell$ is the orbital angular momentum quantum number and $J=\ell+S$ is the total angular momentum quantum number. At the most basic (non-relativistic) level Ps has the same structure as an infinite-mass hydrogenic system, scaled by a factor of two because of the reduced mass $\mu$, where $\mu=m_{1} m_{2} /\left(m_{1}+m_{2}\right)$. Thus $\mu_{\mathrm{Ps}}=m_{e} / 2$ while $\mu_{\mathrm{H}}=$ $0.9995 m_{e}$, and the Ps Bohr radius $a_{\mathrm{Ps}} \approx 2 a_{0}$. Ps wavefunctions are identical to those of the corresponding states in hydrogen, but scaled by the reduced mass factor, which results in Bohr energy levels given by (see any undergraduate atomic physics textbook, for example [171])

$$
E_{n}=\frac{-\alpha^{2} m c^{2}}{4 n^{2}}=\frac{-6.803 \mathrm{eV}}{n^{2}} .
$$

If one looks at the next level of corrections (i.e., of order $\alpha^{4}$ ), however, the similarity between Ps and hydrogen disappears, and we find substantially different energy level structures. The lowest order corrections were first calculated by Pirenne [12]; the shifts to the ground state Bohr 


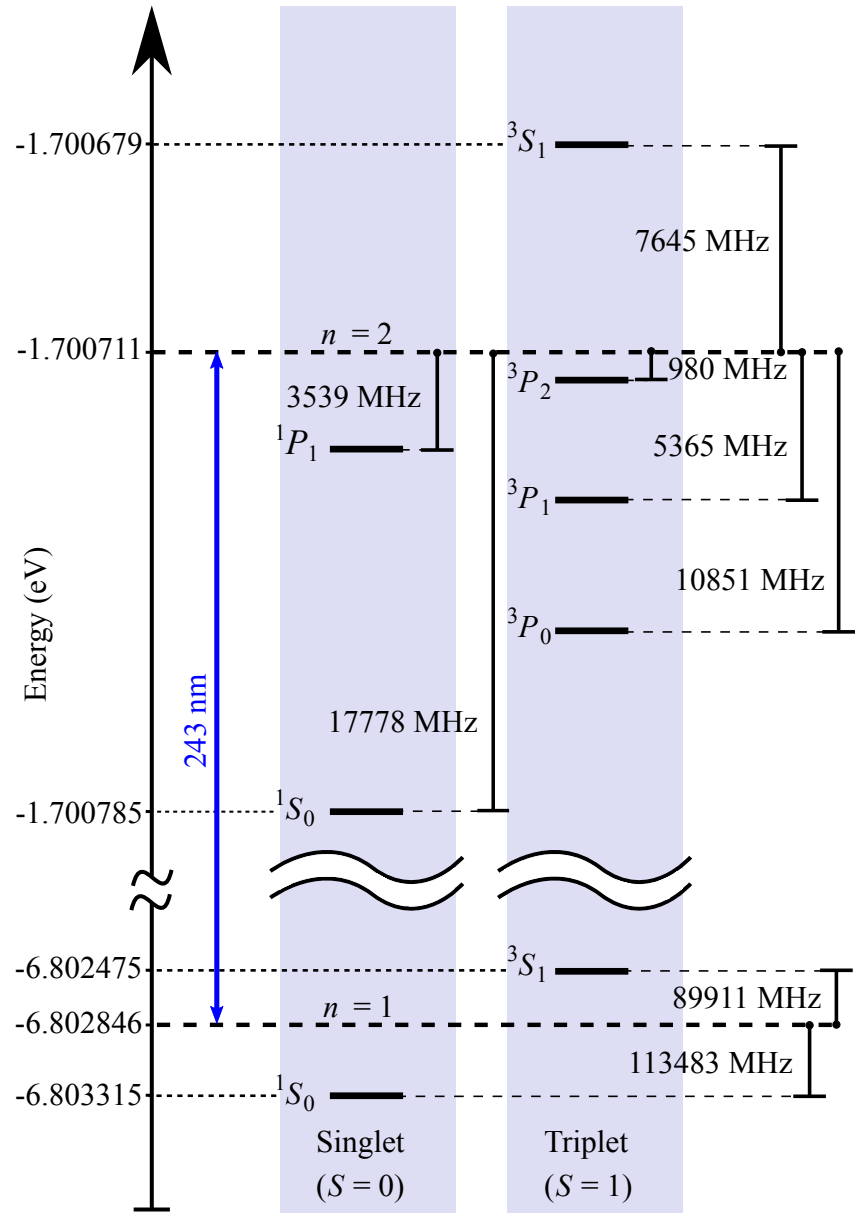

Fig. 1. Ps energy level diagram for $n=2$. The dashed lines indicate the positions of the non-relativistic Bohr energy levels, and the energy intervals indicated are the modern values, summarized in reference [172]. From reference [130].

energy levels (Eq. (1)) can be written as [70]

$$
\begin{gathered}
\Delta E\left(1^{3} \mathrm{~S}_{1}\right)=\alpha^{4} m c^{2}\left(\frac{1}{12}-\frac{5}{64}+\frac{1}{4}\right), \\
\Delta E\left(1^{1} \mathrm{~S}_{0}\right)=\alpha^{4} m c^{2}\left(-\frac{1}{4}-\frac{5}{64}\right) .
\end{gathered}
$$

The first term in these shifts $(1 / 12$ and $-1 / 4$ for the triplet and singlet states, respectively) comes from spinspin interactions between the positron and electron. The common shift (5/64) arises from relativistic corrections to the Hamiltonian (that contribute also to the hydrogen fine structure [148]), and the last term in the triplet case $(+1 / 4)$ is due to virtual annihilation contributions. There is no such contribution to the singlet level at this order because even virtual interactions must obey angular momentum and charge conjugation selection rules; such an effect therefore occurs only at the two-photon level (see Sect. 2.1.2). One can easily verify that the splitting between the ground state singlet $\left({ }^{1} \mathrm{~S}_{0}\right)$ and triplet $\left({ }^{3} \mathrm{~S}_{1}\right)$ levels is given by

$$
E_{\mathrm{hfs}}=E\left(1^{3} \mathrm{~S}_{1}\right)-E\left(1^{1} \mathrm{~S}_{0}\right)=\frac{7}{12} \alpha^{4} m c^{2}=0.84 \mathrm{meV} .
$$

As noted above, this energy difference (which is equivalent to a frequency of $203 \mathrm{GHz}$ ) arises from the interaction between the electron and positron spins $(57 \%)$ and also the existence of virtual annihilation processes (43\%). In hydrogen the former are much weaker, while the latter do not exist, and the corresponding singlet-triplet splitting is therefore considerably smaller $(\sim 1.4 \mathrm{GHz}[148])$. The large contribution to Ps energy levels from mechanisms that exist in hydrogen but are strongly suppressed leads to some inconsistency in terminology. Specifically, due to the weakness of the electron-proton spin interactions, in hydrogen these contributions are referred to as the hyperfine structure, and are much smaller than other contributions at order $\alpha^{4}$. In the case of Ps these interactions are of the same order as the fine structure, as is the annihilation contribution. Thus, Ps does not in fact have a hyperfine structure. Nevertheless, for historical reasons the ground state singlet-triplet energy difference is commonly referred to as a hyperfine splitting (or $E_{\mathrm{hfs}}$ ). This is merely a semantic point that has no bearing on actual Ps physics.

Following the work of Pirenne [12], calculations of the Ps energy level shifts at order $\alpha^{4}$ (again, relative to the Bohr levels) for states with arbitrary values of $n, \ell, S$ or $J$ were also performed by Berestetski [173], with some corrections given by Ferrell [174]. These may be represented as

$$
\Delta E_{n}=\frac{\alpha^{4} m c^{2}}{n^{3}}\left[\frac{11}{64 n}-\frac{(1+\epsilon / 2)}{2(2 \ell+1)}\right]
$$

where $\epsilon=0$ for singlet states $(S=0)$ and for triplet states $(S=1)$,

$$
\epsilon_{\ell, J}=-\frac{7}{3} \delta_{\ell, 0}+\left(1-\delta_{\ell, 0}\right)\left(\begin{array}{c}
\frac{-(3 \ell+4)}{(\ell+1)(2 \ell+3)}, J=\ell+1 \\
\frac{1}{\ell(\ell+1)}, J=1 \\
\frac{(3 \ell-1)}{\ell(2 \ell-1)}, J=\ell-1
\end{array}\right),
$$

where $\delta_{\ell, 0}$ is the Kronecker delta function. The energy level structure of ground state and $n=2$ Ps (using modern values for the shifts [156]) is shown in Figure 1. The theoretical description of Ps energy levels has been advancing steadily and all QED corrections to order $\alpha^{6}$ are now known, as well as some of the higher order contributions [155]. Unfortunately the best experimental data are currently less precise than these calculations, and there is, therefore, a need for improved experimental determinations of Ps energy levels, as discussed in Section 5.2.

As the Ps energy (length) scales are approximately halved (doubled) compared to hydrogen, Ps fluorescence lifetimes, $\tau_{n \ell}$, are twice as long as the corresponding states in hydrogen [148]. Thus, the mean lifetime of $2{ }^{3} \mathrm{P}_{\mathrm{J}} \mathrm{Ps}$ states is $3.2 \mathrm{~ns}$, compared to $1.6 \mathrm{~ns}$ for hydrogen. More generally, the fluorescence lifetime, $\tau_{n \ell}$, of a pure- $\ell$ state 


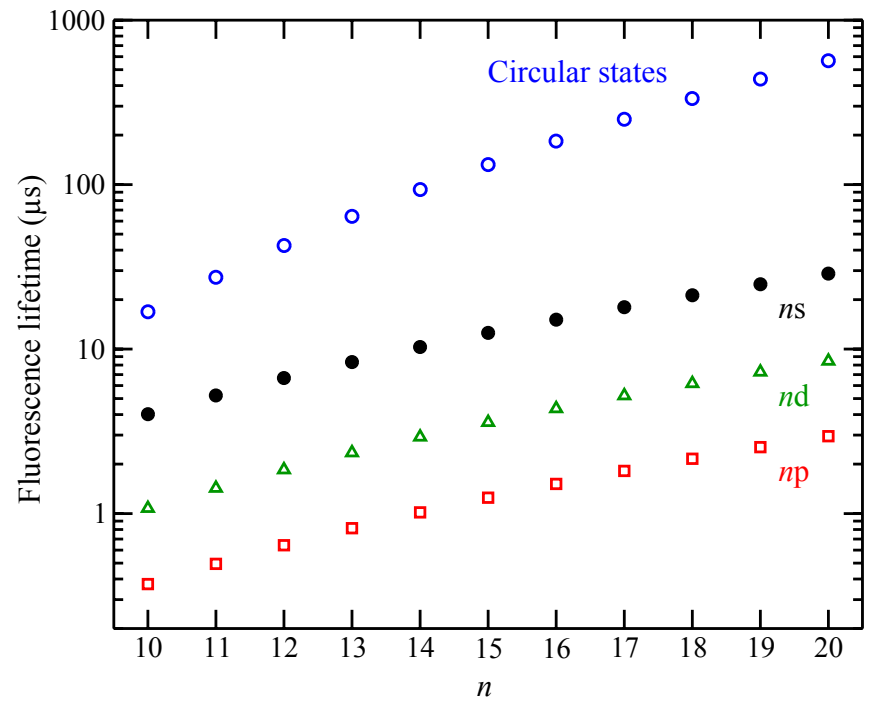

Fig. 2. Fluorescence lifetimes of pure $n \mathrm{~s}, n \mathrm{p}, n \mathrm{~d}$, and circular $\ell=n-1$ Rydberg states of Ps with values of $n$ ranging from 10 to 20. From reference [134].

is given by the inverse of the sum over the Einstein $A$ coefficients associated with all allowed decay pathways to lower-lying states $\left|n^{\prime} \ell^{\prime}\right\rangle$, i.e.,

$$
\begin{aligned}
\tau_{n \ell} & =\Gamma_{n \ell}^{-1} \\
& =\left[\sum_{n^{\prime} \ell^{\prime}} A_{n^{\prime} \ell^{\prime}, n \ell}\right]^{-1},
\end{aligned}
$$

where [175]

$$
A_{n^{\prime} \ell^{\prime}, n \ell}=\frac{2 e^{2} \omega_{n^{\prime} \ell^{\prime}, n \ell}^{3}}{3 \epsilon_{0} h c^{3}} \frac{\ell_{\max }}{2 \ell+1}\left|\left\langle n^{\prime} \ell^{\prime}|r| n \ell\right\rangle\right|^{2}
$$

and $\omega_{n^{\prime} \ell^{\prime}, n \ell}=2 \pi \nu_{n^{\prime} \ell^{\prime}, n \ell}$ is the angular frequency corresponding to the energy difference between the states, $\ell_{\max }=\max \left(\ell, \ell^{\prime}\right)$, and $e, \epsilon_{0}, h$ and $c$ are the electron charge, the vacuum permittivity, the Planck constant, and the speed of light in vacuum, respectively. The fluorescence lifetimes of some pure $\ell$ states and 'circular' $(\ell=n-1)$ states of Ps with values of $n$ in the range 1020 are shown in Figure 2 [134]. As discussed in Section 3.2, pure $\ell$ states of Ps are generally not produced in experiments because the presence of even very weak external electric and magnetic fields can lead to strong $\ell$ mixing [176].

\subsubsection{Ps annihilation}

Unlike most atoms, Ps atoms can decay even when they are in their ground state. This decay process is obviously not the same as radiative decay, and refers instead to the mutual annihilation of the electron-positron pair. One could take an obtuse viewpoint and suggest that the true ground state of Ps is actually the vacuum (or perhaps the so-called Dirac sea), and that the $n=1$ level is in fact the first excited state. Such semantic diversions are not scientifically useful, even if they do make for interesting conversations. Practically speaking, the overall lifetime of an excited positronium atom may depend on both annihilation and radiative decay rates, because annihilation can only occur from certain states (see below), which may only be accessible via radiative transitions.

Ps atoms are composed of two spin $1 / 2$ leptons and thus can be combined to produce states with total spin $S=0$ or $S=1$ (in units of $\hbar$ ). Moreover, since it is made from a particle-antiparticle pair, Ps has no net additive quantum numbers, and is therefore an eigenstate of the Charge conjugation operator $C$ (the operator that reverses all additive quantum numbers and converts particles into antiparticles). Since applying this operator twice must return a particle to its original state, $C$ eigenvalues must be \pm 1 .

Charge conjugation invariance determines the number of gamma rays emitted in Ps decay. This can be understood in a simplified way as follows: applying the $\mathrm{C}$ operator is equivalent to a combined parity and spin exchange operation [177]; exchanging the positron and electron is equivalent to a Parity operation in the center of mass coordinate system, but, since the triplet (singlet) states are symmetric (antisymmetric) under particle spin exchange, we expect this to modify the $C$ eigenvalue by a factor of $(-1)^{S+1}$. Exchanging the electron and positron positions is equivalent to a parity reversal on the relative coordinate wave function, under which the angular part of the spatial wave function contributes a factor of $(-1)^{\ell}$. Finally, particles and antiparticles have opposite intrinsic parity (according to the Dirac theory [5]) so exchanging them gives a factor of $(-1)$. The $C$ parity operator is multiplicative, and the $C$ eigenstate of a state of orbital angular momentum $\ell$ and total spin $S$ is therefore

$$
C=(-1)^{S+1} \times(-1)^{\ell} \times(-1)=(-1)^{\ell+S} .
$$

Photons are also eigenstates of the Charge conjugation operator (they are their own antiparticles), and they must have odd intrinsic parity since the electromagnetic field is reversed under charge exchange. Thus, for one photon $C=$ $(-1)$, and for $N$ photons $C=(-1)^{N}$. The annihilation process is mediated by the electromagnetic interaction, which means that the $C$-parity of the initial state (i.e., a Ps atom) and the final state ( $N$ photons) must be the same, and we have the selection rule [177-179]

$$
(-1)^{\ell+S}=(-1)^{N} .
$$

According to equation (11), $1{ }^{1} \mathrm{~S}_{0}\left({ }^{3} \mathrm{~S}_{1}\right)$ atoms must decay into an even (odd) number of gamma-rays. For free atoms, radiation-less and single-photon decay are suppressed by energy and momentum conservation requirements. These annihilation modes have been observed for thermalized positrons in the presence of a nucleus $[180,181]$ or via in-flight annihilation by energetic positrons $[182,183]$. In general, however, ground state singlet and triplet Ps atoms predominantly decay into two or three photons, respectively.

Note that this selection rule (Eq. (11)) is sometimes attributed to the conservation of angular momentum. This 
Table 2. Annihilation $\left(\tau_{\text {ann. }}\right)$ and fluorescence $\left(\tau_{\text {ff. }}\right)$ lifetimes for the $n=1$ and $n=2$ states of Ps. From reference [130].

\begin{tabular}{lllll}
\hline Level & $\tau_{\text {ann. }}($ ns) & Ref. & $\tau_{\text {fl. }}(\mathrm{ns})$ & Ref. \\
\hline $1{ }^{1} \mathrm{~S}_{0}$ & 0.125 & {$[186]$} & $\mathrm{N} / \mathrm{A}$ & $\mathrm{N} / \mathrm{A}$ \\
$1{ }^{3} \mathrm{~S}_{1}$ & 142 & {$[20]$} & $\gtrsim 10^{16}$ & {$[187]$} \\
$2{ }^{1} \mathrm{~S}_{0}$ & 1 & {$[186]$} & $\simeq 243100000$ & {$[188]$} \\
$2{ }^{3} \mathrm{P}_{0}$ & 100000 & {$[184]$} & 3.19 & {$[148]$} \\
$2{ }^{3} \mathrm{P}_{1}$ & $\simeq \infty$ & {$[184]$} & 3.19 & {$[148]$} \\
$2{ }^{1} \mathrm{P}_{1}$ & 3330000 & {$[185]$} & 3.19 & {$[148]$} \\
$2{ }^{3} \mathrm{P}_{2}$ & 384000 & {$[184]$} & 3.19 & {$[148]$} \\
$2{ }^{3} \mathrm{~S}_{1}$ & 1136 & {$[20]$} & $\simeq 243100000$ & {$[188]$} \\
\hline
\end{tabular}

is incorrect because it is entirely possible for a threephoton state to have zero angular momentum; singlet decay into three photons is not ruled out by momentum considerations [179] (although it would be suppressed relative to two-photon decay by at least a factor of $\alpha$ ). Two-photon emission from triplet states can be ruled out on the basis of angular momentum conservation since in this case the total angular momentum projected along the photon emission direction must be zero or two, which is not compatible with the decay of a triplet Ps atom with a total spin of 1 . Nevertheless, the number of gammaray photons $(N)$ emitted by the self-annihilation of a Ps atom is fundamentally dictated by charge conjugation invariance.

The annihilation of Ps depends on the overlap of the positron and electron wavefunctions. For all practical purposes this means that only states with $\ell=0$ (i.e., S states) will decay by annihilation [184,185]. Because there is a wide-ranging hierarchy of radiative and decay lifetimes that can only be accessed in certain configurations, it is possible to control the lifetime of Ps atoms in a significant way by exciting them with lasers. Table 2 shows the annihilation and radiative lifetimes for states with principal quantum numbers $n=1$ and 2 .

Even for $\mathrm{S}$ states, the annihilation decay rate varies considerably, according to the spin configuration. The annihilation decay rate for singlet states into two photons can be obtained using the Dirac cross section for electronpositron annihilation [5] and the electron (positron) density. The latter can be obtained from the square of the Ps radial wave function at the origin for ground state atoms [171]. This gives

$$
\Gamma\left({ }^{1} \mathrm{~S}_{0}\right)=\frac{\alpha^{5} m c^{2}}{2 \hbar n^{3}} \approx \frac{8 \mathrm{GHz}}{n^{3}},
$$

where $n$ refers to the principal quantum number. The ground state singlet Ps decay rate has been measured to be $\Gamma\left({ }^{1} \mathrm{~S}_{0}\right)=7.990 \pm 1.7 \mathrm{GHz}$ [189], which agrees with QED calculations [190]. If the atoms are created in a laser-field it would be possible to excite ${ }^{1} \mathrm{~S}_{0}$ atoms optically, but in order to do so one needs correspondingly high Rabi frequencies, and thus high intensity lasers. In experiments with a laser tuned to drive $1{ }^{3} \mathrm{~S}_{1} \rightarrow 2{ }^{3} \mathrm{P}_{\mathrm{J}}$ transitions the singlet states would be $200 \mathrm{GHz}$ off resonance and so the singlet atoms are generally neglected and transitions from this state will not be discussed further, although transitions from $n=2$ to the ${ }^{1} \mathrm{~S}_{0}$ state will be.

Since singlet Ps atoms can only decay into two photons, their energy spectra and angular distributions can be easily deduced: in the center of mass frame of the Ps, each photon must have an energy $\mathrm{mc}^{2}$, and the angle between them must be exactly $180^{\circ}$. The $\gamma$-ray energy spectra from the decay of triplet atoms is more complicated as the three photons are constrained to have energies and momenta that together conserve that of the initial Ps atom, and there are many combinations of different photon energies and angles that can satisfy this requirement. Calculating the 3-photon triplet-state decay rate is therefore more complicated because one has to properly account for all possible final photon states. This was first done by Ore and Powell [20] who obtained

$$
\Gamma\left({ }^{3} \mathrm{~S}_{1}\right)=\frac{4}{9 \pi}\left(\pi^{2}-9\right) \frac{\alpha^{6} m c^{2}}{2 \hbar n^{3}} \approx \frac{7 \mathrm{MHz}}{n^{3}} .
$$

Many measurements of the triplet Ps decay rate have been performed [163,191-196], driven by an apparent discrepancy with theory. However, this now appears to have been an experimental artifact related to incomplete Ps thermalization processes; the most recent measurements found $\Gamma\left({ }^{3} \mathrm{~S}_{1}\right)=7.0404(10)(8) \mathrm{MHz}$, in agreement with theory $[82,83,197]$. Incidentally, the singlet and triplet decay rates can be used to determine the ratio of 2 to 3-photon decay processes of positrons in materials (in the absence of Ps formation [20]). This has been measured [198-200] with some small variations observed, which have not yet been fully explained.

Drisko [201] calculated the angular distributions for photons emitted in triplet state decays and showed that the distribution of annihilation gamma rays depends on the azimuthal quantum number $m$. We can immediately see why this must be the case; for $m=0$ decays three photons with helicity $=+1$ are emitted, but the total angular momentum projected along the quantization axis must be zero. This limits the possible angles with which annihilation photons from $m=0$ states can be emitted and still conserve momentum in a different way from the restrictions on the $m=1$ states. The number of photons emitted as a function of angle for the different $m$ states was found to be [201]

$$
\begin{aligned}
N_{m=0} & =C\left[\left(\pi^{2}-9\right) \sin ^{2} \theta+\frac{1}{6}\left(3 \cos ^{2} \theta-1\right)\right] \\
N_{|m|=1} & =C\left[\left(\pi^{2}-9\right)\left(1+\cos ^{2} \theta\right)-\frac{1}{6}\left(3 \cos ^{2} \theta-1\right)\right] \\
N_{\text {total }} & =2 C\left[\left(\pi^{2}-9\right)\right],
\end{aligned}
$$

where the $C$ is a constant of proportionality and $\theta$ refers to the emission angle with respect to the direction of quantization used to define the projection of $m$. Since $N_{m=0}$ and $N_{|m|=1}$ are not the same, if the $m=0$ states are magnetically quenched (see Sect. 2.2.2) then the number of photons detected from long-lived triplet decays may not decrease simply by the factor of $1 / 3$ one would expect from the depletion of one of the three triplet states. In such 
cases the detector position(s) relative to the quantization axis may have to be taken into account.

The decay of Ps atoms can be used to test the underlying symmetries that govern the annihilation process, including CPT and CP and C violations [149,168,202]. Various violations would manifest themselves as forbidden decay modes [39,203-215]. Moreover, because it has no net additive quantum numbers, Ps can in principle couple to Dark Matter or other exotic systems that provide a mechanism for invisible decays [216-219]. Many possible scenarios have been proposed and studied in experiments, including Ps coupling to extra dimensions, unparticles, milli-charged particles, gauge bosons or mirror matter [220-226]. While limits for several of these processes have been set in numerous measurements, no anomalous signals have so far been detected.

\subsection{Extrinsic properties of positronium}

Extrinsic properties of Ps atoms are those that depend on some external factors rather than being fundamental aspects of the Ps system. This could mean Ps velocity or angular distributions, formation efficiencies, or effects due to external fields or interactions with other atoms or molecules (including solid-state materials). Sometimes these descriptions will overlap: for example, the decay rate of a free Ps atom in a particular spin-state is a well-defined intrinsic property, but may be substantially different if the Ps atom is inside a mesoporous film, or if electric or magnetic fields are present.

It is useful to consider some of these properties here because the methods used to produce Ps atoms, and the environments in which they are created, fall into a few commonly used categories. As a result, a broad range of experiments may take place with similar conditions imposed by these methods. Moreover, both the intrinsic and extrinsic properties of Ps are frequently limiting experimental factors, and understanding them helps to understand what experiments are possible, and also what Ps properties are required to move forward: for example the natural linewidth of the $1^{3} \mathrm{~S}_{1} \rightarrow 2{ }^{3} \mathrm{~S}_{1}$ transition is $1.26 \mathrm{MHz}$, determined mostly by the $142 \mathrm{~ns} 1^{3} \mathrm{~S}_{1}$ lifetime (see Sect. 3.3). However, previous measurements of this interval were limited by the available reference line, and by the extent to which the lineshape could be accurately modeled. The reference problem is now moot since frequency combs exist (e.g., [227]). Fully understanding the lineshape and acquiring sufficient statistics, however, are limitations that arise primarily from the extrinsic Ps properties (low number of atoms and high speeds), and not from the intrinsic Ps lifetime. Thus, there is considerable room for improvement in this experiment, if one can obtain an intense cold Ps source.

\subsubsection{Ps production}

Ps atoms can be produced by firing positrons at practically anything, since everything contains large numbers of electrons. Materials that efficiently produce Ps atoms when bombarded with positrons are known as converters, and there are many different types of converter that efficiently produce Ps via several distinct mechanisms. Both the desired Ps properties and other experimental requirements will generally dictate which Ps converter is used. A variety of different converters are listed in Table 3; not all of them are commonly used.

Before slow positron beams became available all Ps experiments were conducted by allowing $\beta^{+}$particles emitted from a radioactive source to interact with a gas [17] or powder [36]. Ps atoms are formed after positrons have thermalized (or partially thermalized) via collisions, which inevitably means that the Ps atoms will then also engage in collisions and may therefore be perturbed [228]. This problem has affected precision lifetime [82] and hyperfine interval measurements [81] but is unavoidable when using this method. Without a detailed knowledge of the collision-induced perturbations, extrapolations to low density cannot be reliably made. Furthermore, this method produces Ps atoms in a relatively large volume, with a large energy spread, and in an uncontrollable manner, and so is not suitable for many experiments, in particular, laser spectroscopy.

Even if perturbing collisions are not an impediment to a particular experiment, and the presence of a background gas has no other detrimental effects, Ps production using a gas target may still be undesirable because it generally results in energetic Ps atoms. This is because of the formation process, in which positrons interact with an atomic or molecular gas $G$ at energies above the Ps formation threshold via the reaction [78]

$$
e^{+}+G \rightarrow \operatorname{Ps}+G^{+} .
$$

The daughter products from this reaction can be in excited states, but Ps is predominantly produced in the ground state [229]. The threshold energy for this reaction is the target ionization energy minus the Ps binding energy $E_{B}$ (6.8 $\mathrm{eV}$ for the ground state in vacuum, see Sect. 2.1.1) but non-thermal positrons with energies up to several hundred eV may still form Ps [230]. Typical ionization energies are of the order of 10's of eV, and Ps produced in this way using $\beta^{+}$particles thus have energies much higher than $E_{B}$. If a slow positron beam is used instead this methodology can generate quasi-monoenergetic Ps beams [231] at lower energies [232] suitable for performing scattering experiments. The Ps energy spread will be determined primarily by that of the positrons; this could range from $\mathrm{meV}$ to eV scales, depending on the positron moderator used.

Ps formation can occur following the interaction of energetic $\beta^{+}$particles with bulk samples of various powders, such as $\mathrm{MgO}, \mathrm{SiO}_{2}$, and $\mathrm{AlO}_{2}$ [35,36,237]. Fast positrons rapidly thermalize in the bulk material, where they are able to form Ps atoms [244]. The large internal surface areas and open volumes of these materials can result in the efficient production of Ps atoms with lifetimes close to the vacuum lifetime. This is because the atoms, which are initially formed in the grains, may subsequently enter the inter-granular voids. They may then interact with the grain surfaces many times without annihilating, which leads to cooling. The Ps atoms are energetically inhibited from re-entering the bulk material, and experience a 
Table 3. Selection of Ps converters, giving some examples of each type, rather than an exhaustive list of all demonstrated converters. The quoted efficiencies $\epsilon$ are the maximum observed for certain optimal conditions (beam experiments only), which may include hot targets. In some cases formation efficiencies, energies, or energy spreads are not explicitly stated in the given reference and are estimated (based on other experiments and/or data) thus, this table is not to be taken too seriously. The given Ps energies $E$ are approximate and may be specific to certain experimental conditions. All data refer to reflection geometry measurements unless otherwise stated. Unknown or undefined parameters are labeled U.

\begin{tabular}{lllll}
\hline Converter & $\epsilon(\%)$ & $\mathrm{E}(\mathrm{meV})$ & Comments & Ref. \\
\hline $\mathrm{Au}$ & 80 & $75 \pm 5$ & Thermal: $E_{a} \approx 0.8 \mathrm{eV}, 2 / 3 \gamma$ & {$[47]$} \\
$\mathrm{Cu}(111)+\mathrm{S}$ & 50 & $140 \pm 30$ & Thermal: $E_{a}=0.74(15) \mathrm{eV}, \mathrm{TOF}$ & {$[52]$} \\
$\mathrm{Ag}(100)$ & 100 & $67 \pm 2$ & Thermal: $E_{a}=0.5(1) \mathrm{eV}, 2 / 3 \gamma$ & {$[233]$} \\
$\mathrm{Al}(111)$ & 25 & $\leq 2600$ & $\phi_{\text {Ps }}$ direct Ps emission, TOF & {$[234]$} \\
$\mathrm{Al}(111)$ & 30 & $80 \pm 5$ & Thermal Ps: $E_{a} \approx 0.85 \mathrm{eV}, \mathrm{TOF}$ & {$[234]$} \\
$\mathrm{Al}(111)+\mathrm{O}_{2}$ & 12 & $8 \pm 1$ & $\mathrm{O}_{2}$ lowers $E_{a}$ to $\approx 0.2 \mathrm{eV}, \mathrm{TOF}$ & {$[235]$} \\
$\mathrm{Ice}$ & 50 & $10000 \pm 2000$ & $2 / 3 \gamma$ & {$[236]$} \\
$\mathrm{MgO}$ powder & $\mathrm{U}$ & $280 \pm 100$ & $\beta^{+}$source, TOF & {$[237]$} \\
$\mathrm{MgO}$ single crystal & 50 & $4000 \pm 1500$ & Single crystal bulk emission, TOF & {$[238]$} \\
$\mathrm{SiO}_{2}$ single crystal/amorphou & $\mathrm{U}$ & 1000 & Surface emission, TOF & {$[239]$} \\
$\mathrm{Mesoporous} \mathrm{SiO}_{2}$ & $30-50$ & $42 \pm 3$ & Transverse component, Doppler & {$[109]$} \\
$\mathrm{SiO}_{2}$ nano-channels & 42 & $\mathrm{U}$ & Oxidized etched Si, TOF & {$[240]$} \\
$\mathrm{Cold} \mathrm{SiO}_{2}$ nano-channels & 2.4 & 12 & Low stability, TOF & {$[241]$} \\
$\mathrm{Si}(100)$ & 100 & $160 \pm 8$ & Excitonic Ps, Doppler & {$[111]$} \\
$5 \mathrm{~nm}$ thick carbon foil & 0.5 & $\geq 10000$ & Transmission geometry, TOF & {$[242]$} \\
190 nm thick Ag(100) foil & 12 & $70 \pm 10$ & Transmission geometry, TOF & {$[243]$} \\
$\mathrm{SiO}_{2}$ on 200 nm substrate & 12 & $100 \pm 5$ & Transmission geometry, Doppler & {$[128]$} \\
$\mathrm{Metal} \mathrm{Organic} \mathrm{frameworks}$ & $\mathrm{U}$ & $250 \pm 10$ & Narrow energy spread, Ry-TOF & {$[124]$} \\
\hline
\end{tabular}

short-range force repelling them from the grain surfaces [245], keeping them mostly in the voids. This concept, with some modification, is important also in modern beam experiments that use thin mesoporous films to produce cold Ps atoms in vacuum [109,246,247], as well as in Ps porosimitry methods $[248,249]$. The work function $\phi_{\mathrm{Ps}}$, of Ps formed in this way is given by

$$
\phi_{\mathrm{Ps}}=-\mu_{\mathrm{Ps}}+E_{\mathrm{bulk}}-E_{B},
$$

where $\mu_{\mathrm{Ps}}$ and $E_{\mathrm{bulk}}$ are the Ps chemical potential and binding energy in the bulk material, respectively. Typical values for the former are a few $\mathrm{eV}$, although they vary widely among materials. $\left|E_{\text {bulk }}\right| \leq\left|E_{\mathrm{B}}\right|$, which explains why it is energetically unfavorable for $\mathrm{Ps}_{\mathrm{s}}$ to re-enter the bulk. In most insulating materials $\phi_{\mathrm{Ps}}$, is negative, and mono-energetic Ps is therefore emitted with material dependent initial energies that can vary from $\approx 1$ to $5 \mathrm{eV}$ (e.g. $[238,239])$. Ps may also be formed from positrons that have not thermalized, in which case the Ps will not be mono-energetic.

The development of slow positron beams $[44,80]$ made it possible to study low-energy positron interactions with surfaces, which in turn enabled new ways of creating Ps to be developed. Using atomically clean surfaces, prepared in UHV conditions, the thermalization and diffusion of positrons in materials and their interactions with surfaces were studied [65]. It has long been known that Ps atoms cannot exist in a high density electron gas, and thus do not exist in the bulk of metals or semiconductors [250,251]. However, positrons implanted into metals or semiconductors can return to the surface [252] and be emitted, either because they have not fully thermalized (epithermal emission), or because the material has a negative positron work function $[48,253,254]$. In either case the positrons may bind with a Fermi-level electron in the surface region and form Ps during the emission process $[52,255,256]$. This is possible because $\left|E_{B}\right|$ is larger than typical electron work functions. This process is known as direct Ps emission, and results in atoms emitted with an energy equal to the Ps "work function", defined as

$$
\phi_{\mathrm{Ps}}=\phi_{+}+\phi_{-}-E_{B},
$$

where $\phi_{+}$and $\phi_{-}$are the positron and electron work functions, respectively. Electron work functions are always positive and generally range from 2 to $6 \mathrm{eV}$, whereas positron work functions can range from approximately -3 up to $+1 \mathrm{eV}$ [65]. Since these are generally small compared to $\left|E_{B}\right|$, direct Ps emission is often one of the main emission channels for positrons that diffuse to surfaces following implantation in metals. The total energy of directly emitted $\mathrm{Ps}_{\mathrm{s}}$ is $\phi_{\mathrm{Ps}}$ (neglecting inelastic scattering processes). The angular distribution, however, may be broad, and contains information regarding the electron momentum distribution. Thus, angle resolved Ps emission spectroscopy can be used to study the electronic structure of the materials from which Ps is emitted [234,255,257]. This process is an analogue of angle resolved photoemission spectroscopy (e.g., [258]) and can be performed with comparable (or superior) energy resolution by exciting emitted Ps atoms to long-lived Rydberg states, as recently demonstrated by Jones and co-workers [137] (see Sect. 3.2). 
Typical values for $\phi_{\mathrm{Ps}}(n=1)$ are several $\mathrm{eV}$ and negative. Conversely, $\phi_{\mathrm{Ps}}(n=2)$ is always positive, and excited state Ps atoms can only be formed in metals by epithermal positrons. This process has been observed for several metals [259-262] and facilitated the first observation of Ps Lyman $\alpha$ radiation [263] as well as microwave spectroscopy of $n=2$ transitions [264-266]. However, this method of producing $n=2$ atoms is generally very inefficient, and is not a viable substitute for laser excitation [139]. Note that technically $\phi_{\mathrm{Ps}}$ is not really a work function (since Ps does not exist inside the bulk) and is sometimes called a formation potential [65,78]; as long as $\phi_{\mathrm{Ps}}$ is defined according to equation (19), this distinction is unimportant.

After implantation in a metal positrons may diffuse back to the surface and become trapped in a surface-state induced by their image potential [267-269]. Positrons trapped in such states can survive for the order of a ns [270]. There are therefore many interactions with surface electrons, and Ps emission can occur, mediated by thermal energy. This process is known as thermal desorption of Ps $[47,52,271-274]$. Here Ps is formed from positrons that are trapped in the near-surface region in a well whose depth $E_{t}$ depends on the surface potential. The activation energy $E_{a}$ needed for a Ps atom to be emitted is given by

$$
E_{a}=E_{t}+\phi_{-}-E_{B}
$$

Typical values of $E_{t}$ and $E_{a}$ are a few $\mathrm{eV}$ and hundreds of meV respectively [272]. The fraction of incident positrons emitted as Ps depends on the temperature $T$ according to [272]

$$
f_{\mathrm{Ps}}=f_{\mathrm{dir}}+f_{\mathrm{th}}(T),
$$

where $f_{\text {dir }}$ is the direct Ps formation fraction (which is largely independent of temperature) and [272]

$$
f_{\mathrm{th}}(T)=f_{\text {surf }} \frac{A \exp \left(E_{a} / k T\right)}{\lambda_{s}+A \exp \left(E_{a} / k T\right)} .
$$

Here $f_{\text {surf }}$ is the fraction of incident positrons that become trapped in a surface potential well, from which thermal desorption may occur. The pre-factor $A$ is a material dependent rate that is related to Ps interactions (scattering and sticking) with the surface [275], and is typically of the order of $\mathrm{PHz}$. In this model thermal Ps emitted according to equation (22) will exhibit an Arrhenius-like activation curve. The Ps emission rate will depend on the details of the surface potential, among other things [275], and hence will be material dependent, and sensitive to surface conditions. Some typical Ps thermal activation curves [272] are shown in Figure 3. Also shown is the fraction of Ps in a beam-Maxwell distribution that has an energy below $25 \mathrm{meV}$ for a given temperature. The actual number of atoms produced that will have this energy will also depend on the thermal activation curve (i.e., on the material) and there will in general be an optimal temperature for maximizing the number of low-energy atoms.

Note that some metals (e.g., Al) may exhibit a decrease in the Ps emission yield at higher temperatures because of thermally-generated defects which may trap positrons and prevent them from returning to the surface [271]. This effect can be minimized if the incident positron impact energy is kept below a few hundred eV. However this may not be desirable because positrons implanted at such low energies will not be fully thermalized, which may result in a reduction in the net yield of Ps atoms emitted in the desired range of low energies. Thermal Ps emitted from a surface will have a beam-Maxwell distribution, similar to that observed for atomic beams emitted from an effusive oven. The angular distributions are not well characterized, and will vary according to the surface properties.

It is possible to modify a metal surface such that the activation energy is lowered, and hence to generate colder Ps. This was demonstrated with $\mathrm{Al}(111)$ at $\approx 100 \mathrm{~K}$ by applying a layer of $\mathrm{O}_{2}$ [235]. Unfortunately the $\mathrm{O}_{2}$ layer has to be kept in pristine condition, and needs careful perpetration and extensive maintenance, especially when used with UV lasers [108]. Thus, despite producing $\approx 10 \mathrm{meV}$ Ps, it is not likely to be used in future optical work.

Activation curves similar to those shown in Figure 3 have been observed for many metals and semiconductors [65,233,271]. Since Ps is not expected to exist in the bulk of any such materials, it was assumed that Ps formation was occurring via the same thermal desorption process in all cases. However, recent experiments have shown that this is not the case: simultaneous measurements of the Ps emission yield and energy from $\mathrm{Si}$ and Ge surfaces has shown that while the yield does exhibit the same Arrhenius type of thermal dependence, the Ps energy is not thermal in nature $[113,114]$. The exact mechanism underlying this process is not yet known but the formation of an exciton-like Ps state (PsX) on the surface has been hypothesized [111], analogous to electronic surface exciton (X) formation [276].

In this model the observed thermal activation is in fact that of electrons thermally promoted to surface states from where they may form PsX. This hypothesis is supported by the fact that the Ps yield can also be increased optically [112]. Both thermal and optical excitation result in the emission of Ps with a nearly constant energy. Indeed, in some cases the Ps energy has been observed to decrease at higher temperatures [113,114], completely ruling out a thermal desorption model. This may be due to a modification of the electronic surface energy levels when there are many electrons present. If so, then it may be possible to modify surfaces so as to produce colder Ps. The observed Ps energies are all in the $100 \mathrm{meV}$ range, and so far no low energy Ps has been found to be produced in this way. The energy of Ps photo-emitted from $\mathrm{Ge}$ at $25 \mathrm{~K}$ was identical to that at $400 \mathrm{~K}$ [131].

It has been known for some time that Ps produced in porous materials may live for an appreciable fraction of its vacuum lifetime, and moreover that the actual lifetimes can, for some range of pore sizes, be used to determine properties of the porous medium [248]. Such investigations can be used to characterize various materials that, for example, may be used as filters, catalysts, or low-k dielectrics [249,277]. Using slow positron beams rather than fast $\beta^{+}$particles, thin films and multi-layered 

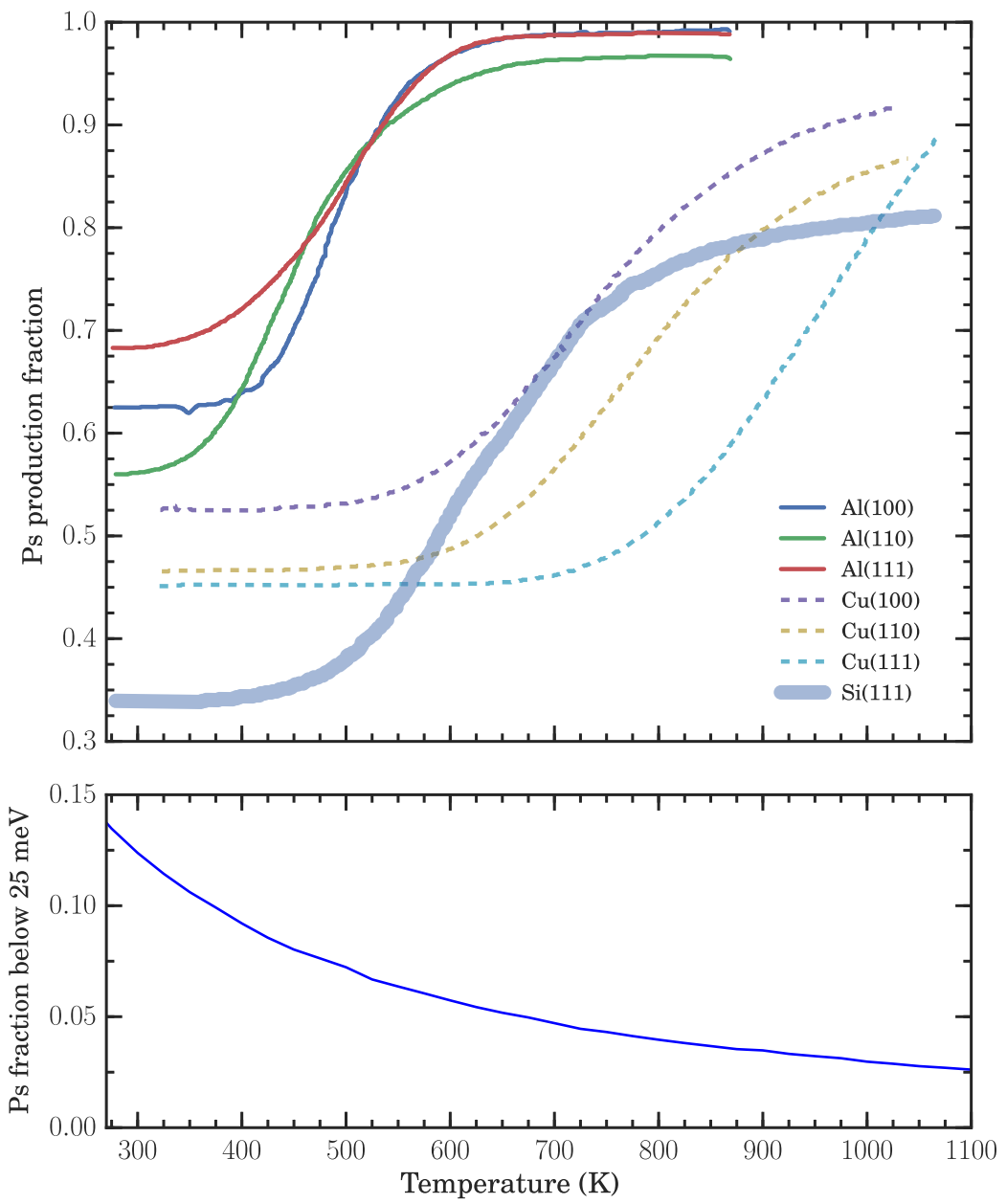

Fig. 3. Ps thermal activation curves for $\mathrm{Al}, \mathrm{Cu}$, and $\mathrm{Si}$, with different crystal orientations as indicated in the legend. These data are based on those in reference [272]. In these data the low-temperature flat part of the curve is due to direct Ps emission, and the temperature dependent part to thermally desorbed Ps (cf. Eq. (21)). The lower panel shows the fraction of a Beam-Maxwell distribution with longitudinal energy below $25 \mathrm{meV}$ as a function of temperature.

structures may be studied [278]. This work has also led to the use of thin mesoporous films that can be used as Ps converters [109,110,246-248,279-284]. These materials produce $\mathrm{Ps}_{\mathrm{S}}$ in a similar way to the powders discussed previously $[35,36]$. They typically have a "swiss-cheese" type of pore structure, comprising interconnected voids with radii ranging from 2 to $8 \mathrm{~nm}$, and porosities close to $50 \%$ or so. Note that we refer to these materials as mesoporous (rather than nanoporous) following the convention of the International Union of Pure and Applied Chemistry: this classification defines microporous, mesoporous and macroporous materials as having pore diameters of less than $2 \mathrm{~nm}$, between 2 and $50 \mathrm{~nm}$, and larger than $50 \mathrm{~nm}$, respectively.

In powders the internal structure often has a fractallike dimensionality [285], and Ps emitted from the grains interacts with the surfaces of granular clusters. In mesoporous films, however, the situation is reversed, and the Ps interacts with the internal void surfaces. Thus, the general formation and cooling mechanisms are similar, but with some important differences. Despite being interconnected, the voids in mesoporous films have a relatively narrow distribution of radii, which means that collisional cooling via Ps interactions with the walls is an efficient process, but also that there is an intrinsic lower limit to the energy of atoms emitted into vacuum because of quantum confinement. That is, the zero-point energy of confined atoms is converted into kinetic energy when they are emitted into vacuum, setting a lower limit to the attainable Ps energy that depends on the average pore size $[109,247,286]$.

The lowest energy state of a Ps atom confined in an idealized spherical pore of diameter $a$ is given by

$$
E_{0}=\frac{\pi \hbar^{2}}{8 m_{e} a^{2}} \approx 750 \mathrm{meV} \times \frac{1}{a^{2}}
$$

where $a$ is in nm. Thus, for $5 \mathrm{~nm}$ diameter pores we would expect to obtain Ps approaching $30 \mathrm{meV}$. This is close to what is observed experimentally [126]. There is generally no obvious thermal component to Ps emitted from porous films since the excited cavity states are mostly inaccessible, although the (unknown) distribution of pore sizes found in high porosity samples may obscure such a signal. 
Because the number of Ps-wall interactions depends on how deep the positrons are implanted into the film, it is possible to tune the Ps energy via the incident positron beam energy [109], but with a lower-level determined by the pore size. This limitation can in principle be avoided by using pore structures that have a long dimension [287], or nano-channels in etched $\mathrm{Si}$ (which operate on a similar principle to the mesoporous films) [240,241].

The mean thermal de Broglie wavelength of Ps at temperature $T$ is given by

$$
\lambda_{\mathrm{dB}}=\sqrt{\frac{h^{2}}{4 \pi m_{e} k T}} \approx 5.3 \mathrm{~nm} \times \sqrt{\frac{100 \mathrm{~K}}{T(\mathrm{~K})}} .
$$

Thus, as Ps diffuses in the porous network and cools down, $\lambda_{\mathrm{dB}}$ will become comparable to the pore size, and there will be a transition from classical diffusion to quantum mechanical tunneling. The latter scenario takes place with a much lower diffusion coefficient than the former [110]. This means that the time it takes for thermalized Ps atoms to be emitted from a mesoporous film can be significantly longer than that of non-thermal atoms. For typical conditions, this can be of the order of 10's of ns if the incident positron beam is implanted at energies $>5 \mathrm{keV}$ or so [126]. This effect may have to be taken into account for some TOF measurements, in which it is generally assumed that the Ps is emitted from the target with negligible delay.

In addition to generating vacuum $\mathrm{Ps}$, the confining nature of porous materials means that they have also found use as Ps "traps", either to study Ps-Ps interactions $[287,288]$ or to look at Ps interactions with (transient) surface species $[131,289]$. The process of quantum confinement is advantageous for such experiments because tunneling Ps becomes localized in a subset of the pore structure, and the effective density of $\mathrm{Ps}_{\mathrm{s}}$ is increased [290]. Similarly, tunneling rates between different accessible pores may have to be taken into account in porosity measurements using lifetime measurements [291,292]. One may also study Ps-gas interactions within such structures via annihilation radiation [293-295], including aerogels [296-298], and using laser spectroscopy it is also possible to study the effects of Ps confinement directly [115].

Recently a new class of porous materials known as metal organic frameworks (MOFs) have been studied with positrons. Some MOFs have been shown to produce Ps in vacuum with very low energy spreads, which occurs because the Ps exists inside the MOF crystals as a delocalized Bloch wave [299,300], and so may have a large effective mass $[301,302]$. This mechanism could potentially allow for the formation of Ps with low energy spreads; for example, Ps with an energy of $250 \pm 10 \mathrm{meV}$ was observed in high-resolution Rydberg TOF measurements [124]. The Ps energy is determined by the energy of the Bloch state, which may be reduced if crystals with larger structures are used. For some experiments involving laser excitation a narrow distribution is useful even for energetic atoms since lasers can then cover the full Doppler spread. An enormous range of MOF materials exist (e.g., [303-305]), and it is not yet clear if they can be reliably used for the efficient production of cold or monoenergetic Ps. However, recent experiments are extremely promising and more data is expected in the near future.

The vast majority of Ps converters operate in a reflection geometry. That is, the Ps is emitted from the same surface that the positrons enter. For many applications, however, it would be convenient to generate positronium on the opposite side to that the positron beam and the Ps experimentation regions can operate independently (cf. [140]). Transmission targets can be produced but are necessarily very thin to allow the positrons to penetrate. This renders them difficult to produce and fragile. Moreover, they are intrinsically inefficient compared to reflection geometries because the stopping profile of a positron beam will follow a Mahkovian like distribution $[252,306,307]$ and it is not generally possible to implant positrons in such a way that more than half of the beam will cross the exit surface. Some transmission targets have been demonstrated. Thin $(100 \mathrm{~nm}) \mathrm{Ag}$ foils heated to $1000 \mathrm{~K}$ work at about $10 \%$ efficiency [243]. It is also possible to produce Ps in transmission from mesoporous structures applied to very thin $(20 \mathrm{~nm})$ substrates, also with approximately $10 \%$ efficiency $[128,308]$. Fast $(10-500 \mathrm{eV})$ Ps can be produced by passing an energetic positron beam through a thin foil of carbon [242], with a few \% efficiency.

In general Ps can be efficiently produced $(100 \%$ is possible) using various types of converters that have been extensively studied over the last 40 years. Ps formation methods developed more recently, specifically mesoporous films, PsX emission from semiconductor surfaces, and delocalized Ps Bloch waves in MOF samples, have been used for many interesting experiments, but have not significantly addressed the problem of generating cold Ps. There is an urgent need to improve the current situation because numerous experiments are substantially limited by the available Ps energies, in particular laser spectroscopy, as discussed in Section 3.

\subsubsection{Ps in electric and magnetic fields}

The theoretical description of Ps energy levels in magnetic fields carries over entirely from the usual hydrogenic theory [148], with some slight modifications. Theoretical considerations concerning Ps in magnetic fields have been discussed explicitly and extensively elsewhere (e.g., [70,309-315]) and will not be discussed here in detail. For ground state atoms the effects of electric fields can be neglected, although in some cases such fields may affect the formation of Ps atoms [32]. In the presence of magnetic fields the Hamiltonian, $\hat{H}$, for Ps atoms can be expressed in the form

$$
\hat{H}=\hat{H}_{0}+\hat{H}_{\mathrm{Z}}
$$

where $\hat{H}_{0}$ denotes the field-free unperturbed Hamiltonian and $\hat{H}_{\mathrm{Z}}$ is the Zeeman Hamiltonian due to the magnetic field $\vec{B}$. If we consider a magnetic field $\vec{B}=\left(0,0, B_{z}\right)$ oriented along the $z$-direction, $\hat{H}_{\mathrm{Z}}$ may be written as

$$
\hat{H}_{\mathrm{Z}}=g_{\left(\mathrm{e}^{-}\right)} \mu_{\mathrm{B}} \hat{s}_{z_{e^{-}}} B-g_{\left(\mathrm{e}^{+}\right)} \mu_{\mathrm{B}} \hat{s}_{z_{e^{+}}} B,
$$


where $\hat{s}_{z_{e^{-}}}\left(\hat{s}_{z_{e^{+}}}\right)$is the projection operator of the electron (positron) spin onto the $z$-axis, $g_{\mathrm{e}^{-}}\left(g_{\mathrm{e}^{+}}\right)$is the electron (positron) spin $g$-factor, where $g_{\mathrm{e}^{-}}=g_{\mathrm{e}^{+}}$, and $\mu_{\mathrm{B}}$ is the Bohr magneton. The fact that the electron (positron) $g$-factor is not exactly 2 (as predicted by the Dirac theory) is of course a consequence of QED corrections and has been measured for both positrons [316,317] and electrons [318,319]; in the latter case extremely high precision has been achieved ( $0.28 \mathrm{ppt})$, yielding $g_{\mathrm{e}^{-}} / 2=$ $1.00115965218073(28)$, in excellent agreement with calculations including tenth order QED terms [320].

If the Zeeman Hamiltonian (Eq. (26)) is diagonalized one obtains the energy shifts of the mixed sublevels levels as a function of the magnetic field in terms of the energies of the unperturbed triplet $\left(E_{t}\right)$ and singlet $\left(E_{s}\right)$ levels [321]:

$$
\begin{aligned}
& E_{1,0}=\frac{\left(E_{t}+E_{s}\right)}{2}+\frac{E_{\mathrm{hfs}}\left(1+x^{2}\right)^{1 / 2}}{2} \\
& E_{0,0}=\frac{\left(E_{t}+E_{s}\right)}{2}-\frac{E_{\mathrm{hfs}}\left(1+x^{2}\right)^{1 / 2}}{2} \\
& E_{1,1}=E_{1,-1}=E_{t},
\end{aligned}
$$

where $E_{\mathrm{hfs}}$ is the hyperfine interval (cf. Eq. (4)), the perturbed levels are labeled according to the total spin $S$ and the projection of the total angular momentum $M_{J}$ as $E_{S, M_{J}}$, and the quantity $x$ is

$$
x=\frac{2 e \hbar B}{E_{\mathrm{hfs}}}=0.276 \times B,
$$

where $B$ is the magnetic field strength in Tesla. The perturbed state energies are given with respect to the mid-point between the singlet and triplet levels only for convenience. One can immediately see (Eq. (29)) that the $M_{J}= \pm 1$ states are unaffected by the magnetic field (see [315] for a discussion concerning a very small diamagnetic effect, that we can ignore). It is also evident that there is no linear Zeeman shift, since $x$ does not appear linearly (Eqs. (27) and (28)). The dependence of the perturbed energy levels on the magnetic field are shown in Figure 4.

The addition of singlet character to the triplet $M_{J}=$ 0 state can significantly reduce its lifetime, and result in two-photon decay. This process, which is sometimes referred to as magnetic quenching, can be utilized in experiments that are sensitive to momentum distributions and rely on two-photon decay processes, such as ACAR or DBS, but involve o-Ps atoms $[79,80]$. The annihilation rate of mixed states will change in proportion to the degree of mixing; in practical terms this has only a minimal effect on the perturbed singlet decay rates, but will increase the perturbed triplet decay rate $\gamma_{1,0}$ by a considerable amount. For magnetic fields $\lesssim 1 \mathrm{~T}$ this is given by (e.g., [322])

$$
\gamma_{1,0}(B) \approx \gamma_{t}+\frac{x^{2} \gamma_{s}}{2}
$$

where $\gamma_{t}\left(\gamma_{s}\right)$ is the unperturbed triplet (singlet) decay rate and $x$ is defined above (Eq. (30)). Thus, in a $1 \mathrm{~T}$ magnetic field the $M_{J}=0$ triplet states will have a mean lifetime of only $3 \mathrm{~ns}$, and will decay predominantly into 2 photons.

Ground state Zeeman mixing was used in the first experiments performed by Deutsch and Dulit to measure $E_{\mathrm{hfs}}[21]$ via changes in the ratio of two and three photon decays in different magnetic fields. The improved version of this experiment measured microwave induced transitions between the perturbed and unperturbed triplet states [23]. As can be observed in Figure 4, in magnetic fields of the order of $1 \mathrm{~T}$ the transition frequency $f_{1,0} \approx 4 \mathrm{GHz}$, which is much easier to produce than the $203 \mathrm{GHz}$ radiation that would be required to drive a direct triplet-singlet transition without Zeeman splitting. For this reason all precision measurements are done using transitions between Zeeman-split triplet states. A handy formula (the Breit-Rabbi equation) giving $f_{1,0}$, the frequency of this transition, is [322]

$$
f_{1,0}=\frac{f_{\mathrm{hfs}}}{2} \times\left[\left(1+x^{2}\right)^{1 / 2}-1\right] \mathrm{GHz} .
$$

A recent experiment [323] has in fact succeeded in driving a direct singlet-triplet transition using a highpower $(300 \mathrm{~W})$ gyrotron and a Fabry-Pérot cavity (with gold mesh mirrors) to produce up to $10 \mathrm{~kW}$ of $203 \mathrm{GHz}$ microwave radiation [324,325]. The basic methodology used in this experiment is similar to the earlier Zeemansplit measurements, except of course for the formidable challenges related to producing intense radiation at the required frequencies [326]. Ps was produced from a radioactive source in a gas cell and the annihilation radiation monitored by scintillator-based detectors. $\mathrm{LaBr}_{3}(\mathrm{Ce})$ scintillators, were used, which have good energy resolution $(\approx 5 \%$ at $511 \mathrm{keV})$ and a short (16 ns) decay time (e.g. [327]); these properties allow for time and energy resolved measurements, and minimize pile up events. NB: $\mathrm{LaBr}_{3}(\mathrm{Ce})$ scintillators would be extremely well-suited to SSPALS measurements (see Sect. 3.1, and cf. [328]), but the cost is prohibitive.

Excited state Ps atoms are much more sensitive to electric and magnetic fields than ground state atoms, primarily because the spacing between energy levels is smaller (see Eq. (5)), and there are more possible angular momentum configurations. Thus, Stark mixing of states of different $\ell$ must be properly described, and Zeeman mixing of spin states will be more significant in weaker fields. Even if no applied electric field is present, it may still be necessary to consider motional Stark effects (especially in the case of Rydberg Ps), since Ps speeds are typically high [132]. It is often the case that magnetic fields are used to guide or trap positrons, and may therefore be unavoidable in some experimental arrangements (although beams can be extracted from magnetic fields if necessary [329]).

Combined Stark and Zeeman effects for Ps states with $n=2$ in magnetic fields of a few hundred Gauss (which is typical of many experimental arrangements) have been calculated by several authors, starting with Kendall [100] and Curry [330]. Higher order corrections and other modifications have been calculated by several researchers [331-335] but, apart from precision hyperfine 
interval measurements, this level of precision is not usually required to interpret experimental data.

To quantify the effects of electric and magnetic fields on Ps decay rates in some recent experiments $[129,130]$, the combined Stark and Zeeman effects for states with $n=2$ have been calculated. These calculations include all singlet and triplet terms, and their associated fine structure, and were performed by determining the eigenvalues and eigenvectors of the complete Hamiltonian matrix in an $\left|n S \ell J M_{J}\right\rangle$ basis, following the convention of Bethe and Salpeter [148]. Here $M_{J}$ is the projection of $\vec{J}$ onto the $z$ axis with which the applied electric and magnetic fields are aligned. The methodology used in these calculations is similar to that of Curry [330], and Dermer and Weisheit [333].

In the presence of parallel electric and magnetic fields, the complete Hamiltonian matrix, $\hat{H}$, for Ps atoms becomes

$$
\hat{H}=\hat{H}_{0}+\hat{H}_{\mathrm{Z}}+\hat{H}_{\mathrm{S}},
$$

where $\hat{H}_{0}$ represents the zero-field (unperturbed) Hamiltonian, $\hat{H}_{\mathrm{Z}}=-\vec{\mu}_{\mathrm{mag}} \cdot \vec{B}$ is the Zeeman Hamiltonian (Eq. (26)) and $\hat{H}_{\mathrm{S}}=-e \vec{F} \cdot \vec{r}$ is the Stark Hamiltonian arising from interactions with an electric field $\vec{F}$ ( $e$ is the electron charge and $\vec{r}$ is the position vector).

To express $\hat{H}$ in matrix form for levels with $n=2$, we consider a $16 \times 16$ matrix. The Zeeman matrix contains off-diagonal elements coupling sub levels with equal values of $\ell$ and $M_{J}$, and values of $S$ that differ by \pm 1 , i.e., the magnetic field couples singlet and triplet terms. The matrix elements between sub levels $\left|n S \ell J M_{J}\right\rangle$ and $\left|n S^{\prime} \ell^{\prime} J^{\prime} M_{J}^{\prime}\right\rangle$ can be expressed as $[130,333]$

$$
\begin{aligned}
\langle & \left.n S^{\prime} \ell^{\prime} J^{\prime} M_{J}^{\prime}\left|\hat{H}_{\mathrm{Z}}\right| n S \ell J M_{J}\right\rangle \\
= & \mu_{\mathrm{B}} B \delta_{\ell^{\prime}, \ell}(-1)^{\ell+M_{J}} \\
& \times\left[(-1)^{S+S^{\prime}}-1\right] \sqrt{3\left(2 J^{\prime}+1\right)(2 J+1)} \\
& \times\left(\begin{array}{ccc}
J^{\prime} & 1 & J \\
-M_{J}^{\prime} & 0 & M_{J}
\end{array}\right)\left\{\begin{array}{ccc}
S^{\prime} & \ell^{\prime} & J^{\prime} \\
J & 1 & S
\end{array}\right\},
\end{aligned}
$$

where $\delta_{x, x^{\prime}}$ is the Dirac delta function, and the term in curved (curly) brackets is a Wigner $3 \mathrm{~J}$ (6J) symbol [336].

In an electric field $\vec{F}=(0,0, F)$ aligned parallel to the magnetic field, the Stark Hamiltonian contains offdiagonal elements coupling sublevels with equal values of $S$ and $M_{J}$, and values of $\ell$ that differ by \pm 1 . The matrix elements between pairs of sublevels take the form [333]

$$
\begin{aligned}
\langle & \left.n S^{\prime} \ell^{\prime} J^{\prime} M_{J}^{\prime}\left|\hat{H}_{\mathrm{S}}\right| n S \ell J M_{J}\right\rangle \\
= & e F \delta_{S^{\prime}, S}(-1)^{S+1+M_{J}^{\prime}} \\
& \times \sqrt{\ell_{\max }\left(2 J^{\prime}+1\right)(2 J+1)} \\
& \times\left(\begin{array}{ccc}
J^{\prime} & 1 & J \\
-M_{J}^{\prime} & 0 & M_{J}
\end{array}\right)\left\{\begin{array}{ccc}
S^{\prime} & \ell^{\prime} & J^{\prime} \\
1 & J & \ell
\end{array}\right\}\left\langle n^{\prime} \ell^{\prime}|r| n \ell\right\rangle,
\end{aligned}
$$

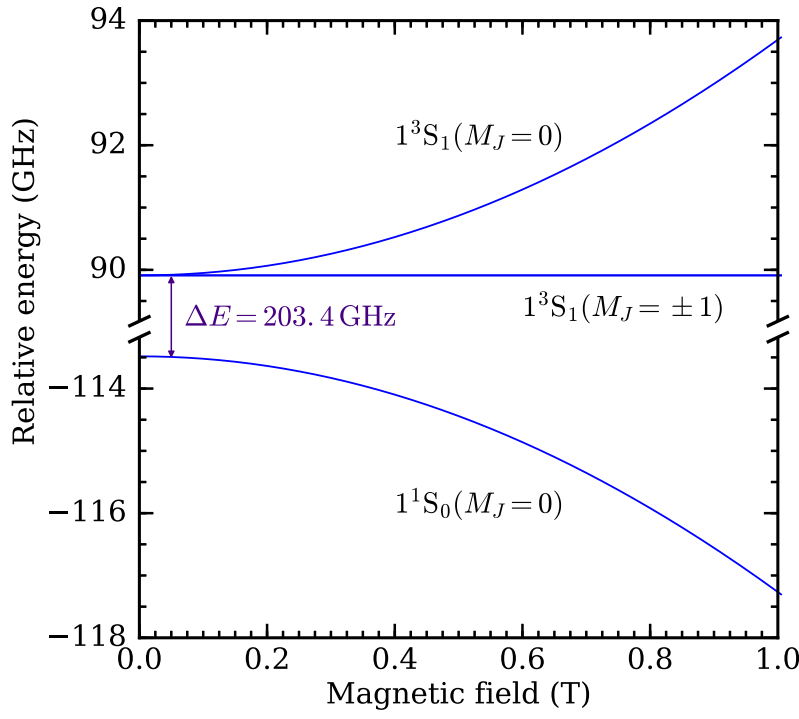

Fig. 4. Zeeman energy shifts of ground state Ps atoms in a magnetic field. The energies are given relative to the Bohr levels as discussed in the text.

where $\ell_{\max }=\max \left(\ell^{\prime}, \ell\right)$, and $\left\langle n^{\prime} \ell^{\prime}|r| n \ell\right\rangle$ is a radial integral. For $n=2$ and $\left|\ell^{\prime}-\ell\right|=1,\left|\left\langle n^{\prime} \ell^{\prime}|r| n \ell\right\rangle\right|=3 \sqrt{3} a_{\mathrm{Ps}}$, where $a_{\mathrm{Ps}}=2 a_{0}$ is the Ps Bohr radius.

Combining the Zeeman and Stark matrices with the diagonal zero-field matrix allows the $n=2$ energy level structure to be determined. This is achieved by calculating the set of eigenvalues, $E_{i}$, labeled with the index $i$, of the complete Hamiltonian matrix for each field strength of interest. Spectral intensities and decay rates can then be obtained from the coefficients, $C_{i, j}$, of the corresponding eigenvectors, where $j$ is an index denoting each $\left|n S \ell J M_{J}\right\rangle$ basis state [130]. Figure 5a shows the calculated Stark shifts for $n=2$ Ps, with a parallel magnetic field of $0.13 \mathrm{mT}$. An expanded region is shown in Figure 5b, indicating the Stark shifts of selected mixed states with and without an applied magnetic field.

Electric dipole selection rules require that for optical transitions from the $1{ }^{3} \mathrm{~S}_{1}$ and $1{ }^{1} \mathrm{~S}_{0}$ ground states to $n=2$ states, $\Delta S=0, \Delta \ell= \pm 1$ and $\Delta J=0, \pm 1(0 \leftrightarrow 0)$ [148]. For experiments in which the laser radiation propagates perpendicular to the magnetic and electric fields, light linearly polarized parallel (perpendicular) to the $z$-axis will drive transitions for which $\Delta M_{J}=0\left(\Delta M_{J}= \pm 1\right)$. Then transitions between states $\left|n S \ell J M_{J}\right\rangle$ and $\left|n^{\prime} S^{\prime} \ell^{\prime} J^{\prime} M_{J}^{\prime}\right\rangle$ will have transition dipole moments, $M_{n^{\prime} S^{\prime} \ell^{\prime} J^{\prime} M_{J}^{\prime}, n S \ell J M_{J}}$, such that [336]

$$
\begin{aligned}
M_{n^{\prime} S^{\prime} \ell^{\prime} J^{\prime} M_{J}^{\prime}, n S \ell J M_{J}}= & (-1)^{J-M_{J}} \sqrt{(2 J+1)\left(2 J^{\prime}+1\right)} \\
& \times\left(\begin{array}{ccc}
J & 1 & J^{\prime} \\
-M_{J} & \Delta M_{J} & M_{J}^{\prime}
\end{array}\right) \\
& \times\left\{\begin{array}{ccc}
\ell & J & S^{\prime} \\
J^{\prime} & \ell^{\prime} & 1
\end{array}\right\}\left\langle 2 \ell^{\prime}|e r| 1 \mathrm{~s}\right\rangle
\end{aligned}
$$

Atoms in highly excited (Rydberg) states are extremely sensitive to external electric fields. In these systems the 
(a)

(b)
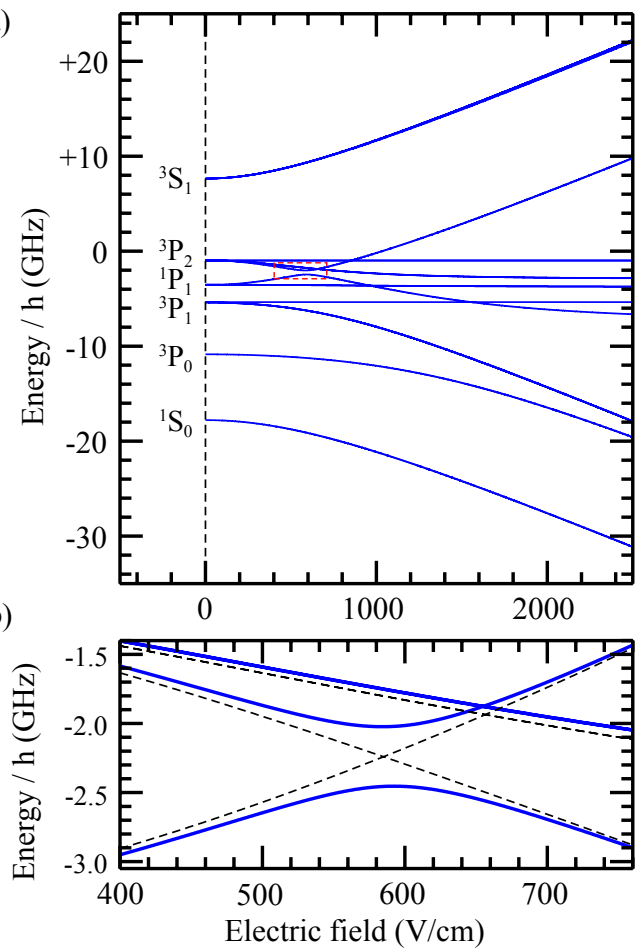

Fig. 5. (a) Calculated Stark energies for $n=2$ Ps in a parallel magnetic field of $B=13 \mathrm{mT}$. The vertical axis shows the energy shift with respect to the $n=2$ energy given by the Rydberg formula. (b) Expanded view of the region at the avoided crossing between the $2{ }^{3} \mathrm{P}_{2}\left(M_{\mathrm{J}}=0\right)$ and $2{ }^{1} \mathrm{P}_{1}$ $\left(M_{\mathrm{J}}=0\right)$ sub-levels highlighted by the red rectangle in (a). The dashed lines in (b) indicate the Stark energies for zero applied magnetic field. From reference [130].

electron is loosely bound to the nucleus (or positron), with an energy scaling of $n^{-2}$ and a large inter-particle separation, scaling as $n^{2}$. Moreover, the $\ell$ degeneracy of hydrogenic atoms leads to total $\ell$ mixing even in very weak external electric fields [176], and so experimentally it is usually the case that fully $\ell$-mixed Stark states are produced. When dealing with such Stark states it is more convenient to describe the atomic structure (that is to say, to solve the Schrödinger equation) using parabolic coordinates. The resulting eigenstates are labeled according to the parabolic quantum numbers $n_{1}$ and $n_{2}$, and the Stark states may then be characterized using the index $k=n_{1}-n_{2}[175]$. For each value of $n$ and the azimuthal quantum number $m$, the allowed values of $k$ range from $-(n-|m|-1)$ to $+(n-|m|-1)$ in intervals of 2 .

In an electric field $\vec{F}=\left(0,0, F_{z}\right)$, the energy shifts of Rydberg Stark states can be expressed analytically to second order as [337]

$$
\begin{aligned}
E_{\text {Stark }}= & \frac{3}{2} n k \text { e } a_{\mathrm{Ps}} F_{z}+\cdots \\
& -\frac{1}{16} n^{4}\left(17 n^{2}-3 k^{2}-9 m^{2}+19\right) \\
& \times \frac{e^{2} a_{\mathrm{Ps}}^{2}}{2 h c R_{\mathrm{Ps}}} F_{z}^{2},
\end{aligned}
$$

where the symbols have their usual meanings, and $a_{\mathrm{Ps}}$ and $R_{\mathrm{Ps}}$ are the Bohr radius and Rydberg constant corrected for the reduced mass of Ps, respectively. Stark shifts for $n=14$ Ps atoms with $m=1$, calculated using this expression, are shown in Figure 6 [336].

Since the energy shift of an electric dipole in an electric field $\vec{F}$ is

$$
E_{\text {Stark }}=-\vec{\mu}_{\text {elec }} \cdot \vec{F}
$$

comparison with equation (37) indicates that, (to first order), an electric dipole moment, $\vec{\mu}_{\text {elec }}$, can be associated with each $k$-state such that [175],

$$
\vec{\mu}_{\text {elec }}=-\frac{3}{2} n k e a_{\mathrm{Ps}}
$$

These large static electric dipole moments allow forces to be exerted on samples in these states using inhomogeneous electric fields [338]. In a spatially inhomogeneous electric field the resulting force is

$$
\vec{f}=\nabla\left(\vec{\mu}_{\mathrm{elec}} \cdot \vec{F}\right)
$$

The magnitude of this force depends directly on the gradient of the electric field. The maximum acceleration that can be achieved using time-independent electric potentials is limited by field ionization of the Rydberg states. The tunnel ionization rate, $\Gamma_{n n_{1} n_{2}}\left(F_{z}\right)$ for a Rydberg-Stark state in an electric field, is given by [337]

$$
\begin{aligned}
\Gamma_{n n_{1} n_{2} m}\left(F_{z}\right)= & \frac{2 h c R_{\mathrm{Ps}}}{\hbar} \frac{(4 C)^{2 n_{2}+m+1}}{n^{3} n_{2} !\left(n_{2}+m\right) !} \\
& \times \exp \left[-\frac{2}{3} C-\frac{1}{4} n^{3} \frac{e a_{\mathrm{Ps}} F_{z}}{2 h c R_{\mathrm{Ps}}}\left(34 n_{2}^{2}\right.\right. \\
& \left.\left.+34 n_{2} m+46 n_{2}+7 m^{2}+23 m+\frac{53}{3}\right)\right],
\end{aligned}
$$

where

$$
C=\frac{1}{e a_{\mathrm{Ps}} \sqrt{2 h c R_{\mathrm{Ps}}}} \frac{\left(-2 E_{n n_{1} n_{2} m}\right)^{3 / 2}}{F_{z}}
$$

and $E_{n n_{1} n_{2} m}=-\left[h c R_{\mathrm{Ps}} /\left(n^{2}\right)\right]+E_{\text {Stark }}$, is the total energy of the state with respect to the ionization limit, in the presence of the electric field. The data in Figure 6 shows the Stark energies for each $k$ state in fields for which the ionization rate exceeds $10^{10} \mathrm{~s}^{-1}$, with the thicker sections at the end of each curve indicating the fields for which the ionization rate ranges from $>10^{8} \mathrm{~s}^{-1}$ to $<10^{10} \mathrm{~s}^{-1}$. It is generally the case that states with negative Stark shifts will ionize (at a given rate) in electric fields that are approximately half the strength of those required to ionize states with positive Stark shifts. The different ionization rates exhibited by states of differing $n$ and $k$ can be used to perform state-selective field ionization using static or pulsed electric fields [338]. 


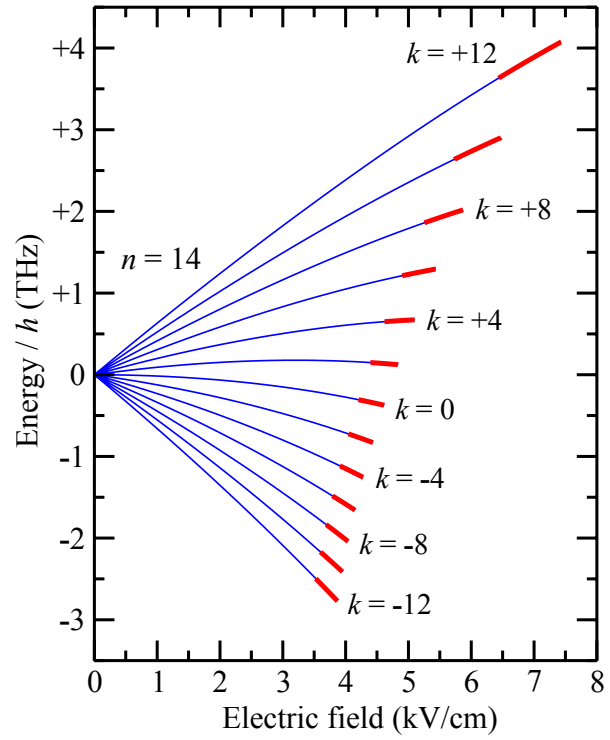

Fig. 6. Stark energies for $n=14$ Ps atoms in electric fields up to $8 \mathrm{kV} \mathrm{cm}^{-1}$. The thicker sections at the end of each curve represent the fields for which the ionization rate ranges from $>10^{8} \mathrm{~s}^{-1}$ to $<10^{10} \mathrm{~s}^{-1}$. From reference [140].

\section{Optical excitation of positronium atoms}

The original suggestion by Mohorovičić [9] was that Ps would have an atomic spectrum similar to that of hydrogen, and that the presence of this system might be detected in suitable astrophysical environments via the observation of the Ps Lyman- $\alpha$ line at $243 \mathrm{~nm}$. As it turned out Ps was first detected in laboratory-based experiments conducted by Martin Deutsch in which he observed gamma radiation following Ps annihilation [17]. Excited states of Ps were observed via Lyman- $\alpha$ light much later by Canter et al. [263], again in laboratory experiments. Ps Lyman- $\alpha$ radiation has not yet been detected from astrophysical sources [339,340], although galactic electron-positron annihilation radiation that can be attributed to Ps has been observed [341].

The first experiments designed to observe the optical excitation of Ps were conducted by Martin Deutsch and his PhD Student Henry Kendall [100] (who went on to win a Nobel prize in 1990 for experiments on proton structure [342]). The Ps excitation attempts were conducted before the invention of lasers and employed a Sn vapor discharge lamp, which emits broad band light down to wavelengths of $\sim 225 \mathrm{~nm}$. Unfortunately the intensity of $243 \mathrm{~nm}$ radiation was not sufficient, and the experiments produced only a small signal that was not statistically significant. A similar experiment was conducted by Varghese et al. [101], also resulting in an ambiguous signal. A complete history of Ps-laser experiments (including molecular and ionic $\mathrm{Ps}$ ) is given in Table 1. Without question this table will already be out of date when this article is published.

Although many established techniques developed in laser spectroscopy can be directly applied to Ps experiments, this is not always the case. While comparable number densities can be produced [121], the absolute number of Ps atoms available is usually much lower than is the case for other atomic or molecular species. Thus, in a typical gas cell (for example) the total number of atoms or molecules present is likely to be at least of the order of $10^{18}$, whereas in Ps experiments it is more likely that a only few million atoms (or fewer) will be present. In general this precludes the use of techniques based on measuring light absorption (e.g. [343]) since, even for complete absorption, with such low numbers of atoms this amounts to an undetectable laser attenuation of the order of 1 part in $10^{10}$. Other techniques, however, are applicable to Ps-based experiments: for example, photoionization-based methods work with similar efficacy. Moreover, electronpositron annihilation can be exploited for various useful detection schemes. These can be more flexible and/or convenient since they generally do not require any in-vacuum modifications, and detectors can be placed in any position outside the vacuum system.

The first laser induced excitation of Ps was a twophoton $1^{3} \mathrm{~S}_{1} \rightarrow 2{ }^{3} \mathrm{~S}_{\mathrm{J}}$ transition, driven by a pulsed dye laser by Chu and Mills in 1982 [102]. This experiment was upgraded some years later to incorporate improved detection methods and a CW laser, finally achieving a measurement with an uncertainty of only 2.6 parts per billion $(3.2 \mathrm{MHz})$ [107] (see Sect. 3.3).

Almost a decade after the first experiments of $\mathrm{Chu}$ and Mills, single photon $1{ }^{3} \mathrm{~S}_{1} \rightarrow 2{ }^{3} \mathrm{P}_{\mathrm{J}}$ transitions in Ps were observed at Lawrence Livermore national laboratory (LLNL), also using a pulsed dye laser [104]. Using a second dye laser these researchers were also able to produce highly excited (Rydberg) Ps states for the first time [105] using a two-step $1^{3} \mathrm{~S} \rightarrow 2^{3} \mathrm{P} \rightarrow n^{3} \mathrm{~S} / n^{3} \mathrm{D}$ excitation process (see Sect. 3.2). The next experiments in which Ps atoms were excited with lasers did not take place until 2010 [109] (see Tab. 1).

Thus, even though it has been possible to produce Ps with near thermal energies in the laboratory since the late 1970 s, by 2009 only two sets of experiments had been performed in which Ps was optically excited. One of the reasons for this was the limited availability of pulsed positron sources. In the context of recent experiments it is worthwhile considering the basic requirements for optical excitation of Ps. A straightforward example of such an experiment is exciting the single photon $1^{3} \mathrm{~S}_{1}-2^{3} \mathrm{P}_{\mathrm{J}}$ transition. The energy interval between these levels (see Eq. (1)) is $3 / 4 \times 6.8 \mathrm{eV}=5.1 \mathrm{eV}$, which corresponds to light with a wavelength $\lambda=243 \mathrm{~nm}$. The cross section for this excitation can be crudely approximated as $\lambda^{2}$, and the laser fluence required to saturate the transition will be roughly $h c / \lambda^{3}$, or $1 \mathrm{~nJ} / \mathrm{cm}^{2}$. If the time-scale for the excitation is on the order of the $2 \mathrm{P}$ lifetime (3.2 ns), then a CW laser would have to have a fluence of a few hundred $\mathrm{mW} / \mathrm{cm}^{2}$ to saturate the transition, which is well within the capabilities of modern diode lasers, and even in 1980 could have been easily obtained.

However, the laser intensity is not the only consideration affecting the feasibility of a scheme to optically excite Ps. All practical methods of Ps production invariably result in energetic Ps atoms, with thermal (or higher) energies (e.g. [52,109]). Because of its very low mass, thermal Ps atoms have speeds of the order of $7 \times 10^{4} \mathrm{~m} \mathrm{~s}^{-1}$. 
For a Ps gas with a Maxwell-Boltzmann distribution this would lead to Doppler broadening (e.g., [171]) such that

$$
\frac{\Delta \nu}{\nu}=2 \sqrt{\ln 2} \frac{v}{c} \approx 4 \times 10^{-4} .
$$

Thus, for $1^{3} \mathrm{~S}_{1}-2^{3} \mathrm{P}_{\mathrm{J}}$ transitions $(\nu=1.234 \mathrm{PHz})$ the $50 \mathrm{MHz}$ natural linewidth (arising from the $2 \mathrm{P}$ lifetime) will be irrelevant, since the transition will be Doppler broadened to almost $500 \mathrm{GHz}$. This means that a broadband laser is required to address the entire population of Ps atoms. However, the applied laser fluence will then be spectrally "diluted" by a factor of $\eta \approx 50 \mathrm{MHz} / \Gamma_{\text {laser }}$, where $\Gamma_{\text {laser }}$ is the bandwidth of the excitation laser. In order to excite all atoms this should match the Doppler broadened width of the transition $(\eta=1 / 10000)$, but in practice is often limited to $\sim 100 \mathrm{GHz}$ or so $(\eta=1 / 2000)$. Then, instead of requiring a $300 \mathrm{~mW} / \mathrm{cm}^{2} \mathrm{CW}$ laser, one would need $600 \mathrm{~W} / \mathrm{cm}^{2}$, which is much more difficult to achieve.

It is possible to use a low power laser and a Fabry-Pérot cavity to achieve high laser-light intensities from a CW laser (e.g. [227]). This approach is beneficial in precision spectroscopy, where nonlinear effects present in intense pulsed lasers must be taken into account [344], and was in fact used for the most accurate measurement of the $1{ }^{3} \mathrm{~S}_{1} \rightarrow 2{ }^{3} \mathrm{~S}_{1}$ transition [107] (see Sect. 3.3). Since they can be difficult to stabilize and are often incompatible with other experimental requirements this methodology is not widely used in Ps research; the only other example in addition to the precision experiments of Fee et al. [107] is that of reference [123]. High instantaneous laser intensities can be produced relatively easily using pulsed lasers. Dye lasers are well-suited for this purpose [102,104,109], although other systems have also been used $[127,135]$. Dye lasers can easily produce hundreds of micro Joules in pulses a few $\mathrm{mm}$ wide and $5 \mathrm{~ns}$ in duration. This amounts to fluences in the $\mathrm{MW} / \mathrm{cm}^{2}$ range. In order to take advantage of pulsed lasers in this way, however, one also needs a pulsed positron beam.

The generation of DC positron beams with currents of the order of $10^{6} \mathrm{e}^{+} \mathrm{s}^{-1}$ has enabled detailed studies of positron-surface interactions [65], but does not necessarily facilitate the production of intense pulsed beams using standard bunching techniques (e.g. [80]). The initial experiments of Mills and $\mathrm{Chu}$ used an ingenious magnetic bottle bunching technique $[102,345]$ which was able to provide $\approx 10$ ns wide positron pulses containing 10 's of positrons. Later work utilized an intrinsically pulsed beam derived from a microtron accelerator [346]. This device produced $16 \mu$ s wide pulses that were bunched into $10 \mathrm{~ns}$ wide pulses containing $7 \times 10^{4}$ positrons.

Similarly, the LLNL experiments mentioned previously were conducted using a high intensity linac [347]. In the 1980s and 1990s this accelerator (now decommissioned) generated the most intense positron beam in the world, with a reported average current of $3 \times 10^{9}$ positrons/second [347]. In the short-pulse mode around $10^{6}$ positrons were produced in $20 \mathrm{~ns}$ pulses at $1440 \mathrm{~Hz}$. Having been built in the 1960s for neutron cross-section measurements (to support nuclear weapons research) the beam dump was located in a heavily shielded underground cave in which very high levels of radiation could be safely generated. This, and the high power of the machine, made it ideal for generating intense positron pulses. Other positron beams have been constructed using linacs, but so far none have been able to match the intensity of the LLNL beam (e.g. [348-350]). Accelerator-based beams of this type are generally large and expensive, and are not widely available. Thus, the LLNL experiments $[104,105]$ remain the only ones in which the intrinsically pulsed output of a linac was utilized for Ps excitation experiments with pulsed lasers (although Ps ion photodetachment has been performed in this way [116]).

A significant breakthrough was the development of the Surko type buffer gas positron trap $[66,67,69]$ and nonneutral plasma [351] compression techniques [352-355]. These advances allow a typical DC positron beam generated from a neon moderator [53] to be turned into a pulsed beam [345] having characteristics suitable for producing Ps that can be addressed by pulsed lasers [356,357]. It was the application of the Surko trap that opened the door to a new program of Ps-laser interactions starting in 2010 [109], leading to many subsequent experiments, as indicated in Table 1.

Another development that facilitated Ps-laser experiments was that of single-shot positron annihilation spectroscopy (SSPALS). The time $[17,248]$ or energy $[17,271$, $273,358]$ distributions of annihilation photons can be used to detect the production of Ps atoms, but most detection systems are designed to observe one event at a time [7880]. SSPALS is a technique wherein annihilation gamma radiation is measured in "real time", so that lifetime spectra can be measured via many photons produced in a single intense positron pulse. In this way laser-induced changes in Ps decay rates can be observed. To accomplish this many thousands of $\gamma$ ray photons are recorded continuously over a time span of around $1 \mu \mathrm{s}$; ordinary single-event detection is extremely inefficient compared to SSPALS when intense positron pulses are used.

Single shot lifetime spectra are characterized using the delayed fraction parameter $f_{\mathrm{d}}$, which is defined as

$$
f_{\mathrm{d}}=\int_{B}^{C} V(t) \mathrm{d} t / \int_{A}^{C} V(t) \mathrm{d} t,
$$

where $V(t)$ is the detector anode voltage. This parameter is proportional to the amount of long-lived Ps present, but should not be mistaken for the actual Ps fraction [113]. The integration regions defined by $A, B$ and $C$ are selected according to the type of detector used and the processes being studied. The values of $A$ and $C$ are largely determined by the times over which annihilation radiation can be observed, whereas the choice of $B$ depends on the system under study. For fast processes (e.g., photoionization) one might use $B=3 \times \tau$, where $\tau$ is the scintillator decay constant, to ensure that a reasonable fraction of the integrated spectrum is due to Ps decay, and not delayed light from the prompt peak. For the optimal observation of processes that occur on different time scales one would use different values of $B$, and the interpretation of $f_{d}$ should 
then be modified; rather than a measure of the amount of long-lived ground state Ps present, $f_{d}$ would then represent some arbitrary integration of the lifetime spectra that happens to be the most sensitive to changes induced in the spectra by certain events. Such changes are characterized by the signal parameter $S_{\gamma}$, given by

$$
S_{\gamma}=\left(f_{\mathrm{bk}}-f_{\mathrm{sig}}\right) / f_{\mathrm{bk}},
$$

where $f_{\mathrm{bk}}$ refers to the background Ps decay rate. This is the value of $f_{\mathrm{d}}$ obtained without the perturbing influence being studied, and $f_{\text {sig }}$ is the value obtained when it is present. In some experiments (e.g. [125]) a two-step $1^{3} \mathrm{~S} \rightarrow 2{ }^{3} \mathrm{P} \rightarrow n^{3} \mathrm{~S} / n^{3} \mathrm{D}$ excitation scheme is used. Then $f_{\text {sig }}$ refers to the presence of both lasers, tuned to the relevant frequencies, and $f_{\mathrm{bk}}$ refers to the case where the laser driving the second transition is tuned off resonance. This can result in some $n=2$ atoms decaying via quenching mechanisms instead of being excited to Rydberg levels, giving a distorted background signal.

Examples of single shot lifetime spectra are shown in Figure 7 . These data were recorded using two different detectors: one incorporating lead tungstate $\left(\mathrm{PbWO}_{4}\right)$ and one using cerium doped lutetium yttrium oxyorthosilicate $\left(\mathrm{Lu}_{2(1-x)} \mathrm{Y}_{2 x} \mathrm{SiO}_{5}: \mathrm{Ce}\right)$, abbreviated as PWO and LYSO respectively. As discussed elsewhere [328], both of these materials can be used for SSPALS measurements. PWO is less efficient in terms of light production (a few percent of NaI) [359] and has a decay time of around $10 \mathrm{~ns}$, which makes it well suited for very high intensity pulses, as are required for $\mathrm{Ps}_{\mathrm{S}}-\mathrm{Ps}_{\mathrm{s}}$ scattering or $\mathrm{P}_{\mathrm{S}_{2}}$ formation experiments [119,360-362]. LYSO has a high density $\left(8 \mathrm{~g} \mathrm{~cm}^{-3}\right)$, a high light output ( $\sim 75 \%$ of $\mathrm{NaI})$, and a decay time of $\approx 40$ ns. These properties mean that LYSO works well as a scintillator in positron emission tomography (PET) [363] cameras. It is also useful in SSPALS; although the decay time is fairly long compared to the $140 \mathrm{~ns}$ Ps lifetime, the excellent statistics arising from the high light output mean it can give a signal comparable to those obtained from PWO. For the detection of long-lived events, however, (e.g., following the production of Rydberg Ps) the decay time is less important, and LYSO based detectors can provide a much improved signal to noise ratio, compared to PWO [328].

The experiments performed by Ziock and co-workers described above utilized a pulsed dye laser with a few hundred $\mu \mathrm{J} / \mathrm{cm}^{2}$ per pulse and a bandwidth of $\approx 360 \mathrm{GHz}$ $[104,105]$. In these experiments a linac was used to produce $\approx 10^{5}$ positrons per pulse at a repetition rate of $1440 \mathrm{~Hz}$. These positrons were implanted into a $1000 \mathrm{~K} \mathrm{Cu}$ target, which emitted thermal Ps atoms with an efficiency of $\approx 40 \%$. In this work the optical saturation of $1{ }^{3} \mathrm{~S}_{1} \rightarrow 2{ }^{3} \mathrm{P}_{\mathrm{J}}$ transitions [104] and the production of Rydberg Ps was observed [105].

Despite employing an intense pulsed positron beam, an efficient source of Ps atoms, and a high-power broadband pulsed laser, the signal observed in the LLNL experiments was extremely weak, primarily because of the detection method used; Ps annihilation was monitored using a system designed for TOF measurements that consisted of a collimated plastic scintillation detector whose field of

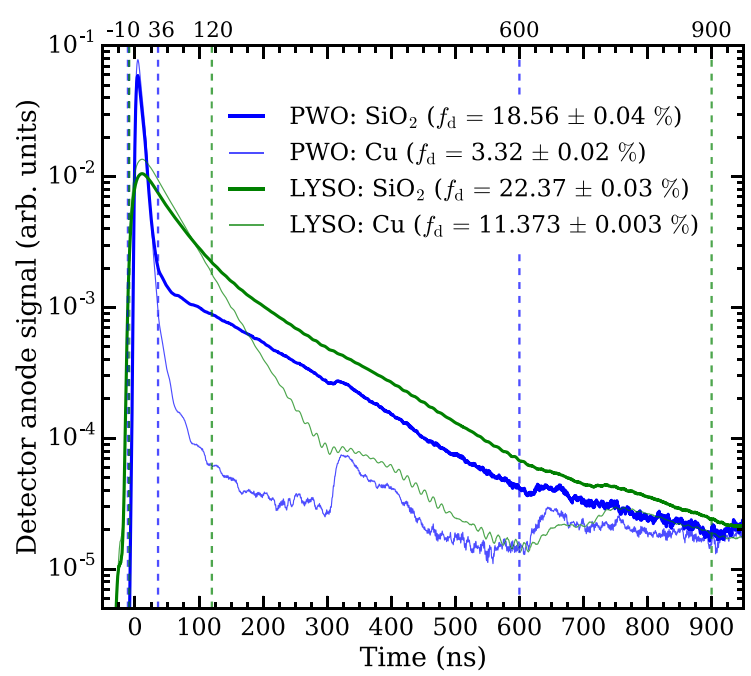

Fig. 7. Examples of SSPALS lifetime spectra recorded using both PWO and LYSO detectors. The dashed lines indicate regions of integration used to obtain the delayed fraction $\left(f_{d}\right)$ numbers indicated in the panel. $\mathrm{SiO}_{2}$ and $\mathrm{Cu}$ refer to the silica converter target and part of an untreated copper target holder. The former is expected to generate around $25 \%$ positronium, and the latter only a few percent, illustrating the difference in the lifetime spectra when Ps is produced. Reprinted from [328] with permission from Elsevier.

view was offset from the target by about $1 \mathrm{~cm}$ so as to reduce background events from the target [364]. The excitation laser was therefore also separated from the target, resulting in around $50 \%$ of the Ps atoms being lost due to annihilation in flight. This, along with spreading out of the Ps, and the total the o-Ps production efficiency $(\sim 60 \%)$ and the excitation efficiency probably resulted in the generation of a few thousand excited atoms per linac pulse. However, the TOF detector used was designed to measure single events, and so only one event could be observed per linac pulse.

More efficient detection could have been obtained, either by directly measuring the constituents of Ps atoms (i.e., electrons or positrons) following ionization, as in the measurements of Chu and Mills [102], or by using a technique like SSPALS [365]. For example, a $\gamma$-ray detector with only $20 \%$ solid angle coverage and $50 \%$ intrinsic detection efficiency would have recorded on the order of $10^{4}$ photons per pulse, and a spectrum that took 1 week to record could have been recorded in minutes. Using a two-stage Surko trap [366] that produces $\approx 10^{5}$ positrons per pulse at $1 \mathrm{~Hz}$ it is possible to record high quality Ps excitation data using SSPALS. The positrons can easily be compressed in time to a few ns [345], and rotating electric field techniques can compress the beam width to a few $\mathrm{mm}$ or less, even if the positron density is not in the plasma regime $[356,367,368]$. The application of all these techniques together opens the door for a wider variety of positronium-laser experimentation, without the requirement of a large and expensive accelerator-based positron beam.

The following discussion is divided into sections based on three different processes: namely, single-photon 
$1^{3} \mathrm{~S}_{1} \rightarrow 2^{3} \mathrm{P}_{\mathrm{J}}$ transitions, two-step two-color excitations to Rydberg states: $1{ }^{3} \mathrm{~S} \rightarrow 2^{3} \mathrm{P} \rightarrow n^{3} \mathrm{~S} / n^{3} \mathrm{D}$, and twophoton $1{ }^{3} \mathrm{~S}_{1} \rightarrow 2^{3} \mathrm{~S}_{1}$ transitions. These encompass the main areas of Ps-laser physics currently being pursued. Lyman- $\alpha\left(1^{3} \mathrm{~S}_{1} \rightarrow 2^{3} \mathrm{P}_{\mathrm{J}}\right)$ transitions are the workhorse of many experiments since this is the easiest way to get atoms out of the short-lived ground state. This can be done in order to perform Doppler spectroscopy, or to look at level-shifts, to study the effects of external fields on excited ( $n=2$ states) or even to produce cold positrons by photoionization of Ps atoms. It is also the basis for the Rydberg excitation experiments. Two-photon $1{ }^{3} \mathrm{~S}_{1} \rightarrow$ $2{ }^{3} \mathrm{~S}_{1}$ transitions are rather different as they are in general best suited for precision experiments. Just as in hydrogen, the excited $2{ }^{3} \mathrm{~S}_{1}$ levels are radiatively metastable, and thus amenable to precision measurements. Of course, in the Ps case there is also an annihilation channel, which significantly broadens the line, but this limit has not yet been reached experimentally.

\subsection{Single photon $1^{3} \mathrm{~S}_{1} \rightarrow 2^{3} \mathrm{P}_{\mathrm{J}}$ transitions}

In general, the difficulties associated with performing a single photon $1{ }^{3} \mathrm{~S}_{1} \rightarrow 2{ }^{3} \mathrm{P}_{\mathrm{J}}$ excitation of Ps atoms are primarily associated with (1) not having enough atoms and (2) the atoms you do have being too hot. The latter of course results in Doppler broadening, and in practice limits the overall excitation efficiency one can obtain, according to the intensity and bandwidth of the available laser radiation. As mentioned above, the intensity limitations can be solved by using a pulsed positron beam, so that the Ps production can be temporally matched to the light output of a pulsed laser. The bandwidth limitations can be addressed in various ways, such as using dispersive media in the laser resonator [227] to produce broad-band laser light. This approach is trivially easy to implement with a standard dye laser, but results in an incomplete spectral profile, and thus requires even more power than the spectral dilution would suggest. Another solution is simply to use solid-state laser systems that can be made to be intrinsically broad-band $[127,135]$.

It is now relatively straightforward to produce $n=2$ Ps atoms. As discussed in Section 2.2.2, these excited states are susceptible to the types of electric and magnetic fields likely to be present in a positron trap environment. Thus, it is extremely important to properly understand Stark and Zeeman effects in many experiments. Even in a two-step excitation process where the atoms are driven to high-lying states, the effects of external fields on the intermediate states can be detected in the resulting lineshapes. Moreover, singlet-triplet coupling via the Zeeman effect can provide a pathway to short lived states, and thereby provide a loss and/or detection mechanism. Experimentally it is non-trivial to change the magnetic field strength over a wide range because it is used to guide the positron beams, and typical coils only produce fields up to a few hundred G. However, electric fields can be controlled over a wider range. This is accomplished using electrodes to define the electric field in the region where Ps atoms are optically excited, as shown in Figure 8. This arrangement is well suited for studies of Stark effects in $n=2$ Ps. (a)
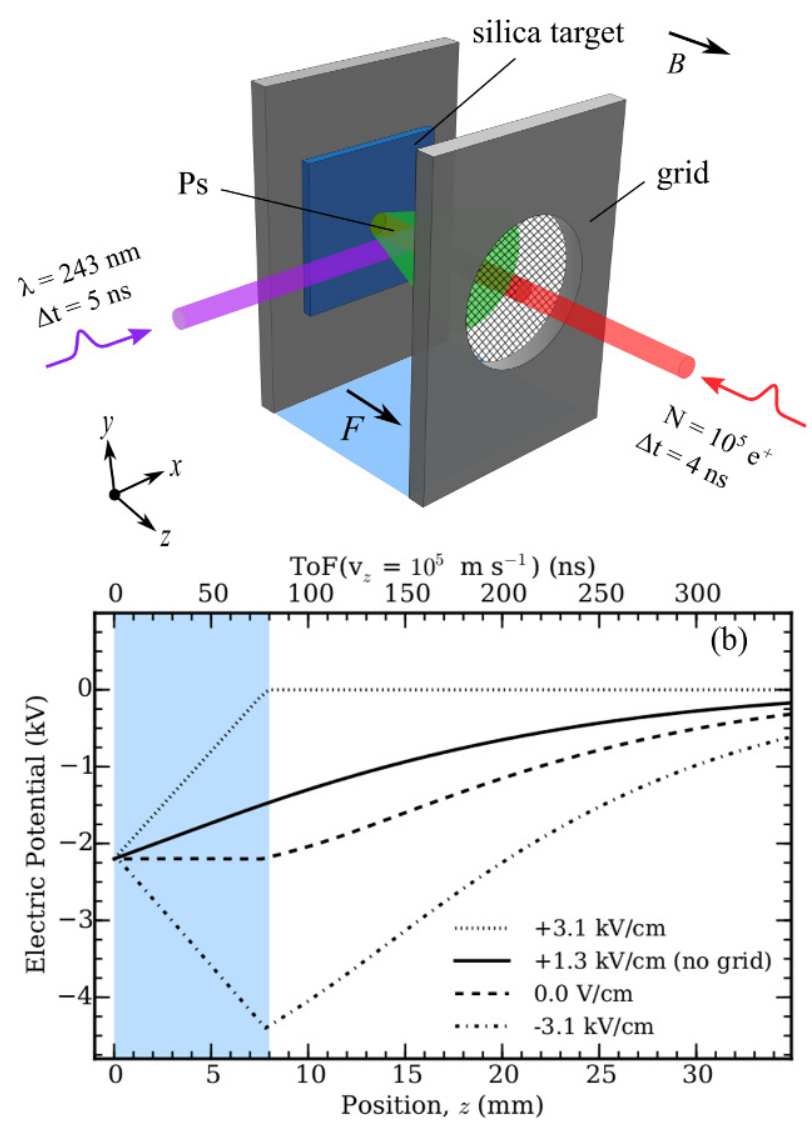

Fig. 8. (a) Schematic representation of the electrode structure used to produce $n=2$ Ps atoms and (b) calculated electric potentials in the target region. A pulsed positron beam is implanted into a silica target which emits Ps atoms between two electrodes that define the electric field in the excitation region. The legend in (b) indicates the magnitude and direction of the electric field in the area between the target and the grid (indicated by the shaded region), and the flight time for Ps with a velocity of $v_{z}=10^{5} \mathrm{~m} \mathrm{~s}^{-1}$ is shown on the top axis. From reference [130].

\subsubsection{Stark and Zeeman effects in $n=2 \mathrm{Ps}$}

Because the radiative and annihilation decay rates of different Ps levels cover an enormous range (see Sect. 2.1.2 and Tab. 2), the application of external fields can have a dramatic effect on Ps decay rates $[129,130]$. Processes in which Ps decay rates are increased via singlettriplet (Zeeman) mixing are known generally as magnetic quenching (MQ). MQ can occur via direct mixing of $m=0$ ground states (see Section 2.2.2) as was observed in some of the first experiments conducted with Ps [21,29,30,309], or via mixing of excited states. In the latter case Ps may annihilate directly from the excited state (if it has sufficient $2{ }^{1} \mathrm{~S}_{0}$ character), or atoms initially in triplet states may be excited to a Zeeman-mixed state that has both singlet and triplet components, and subsequently decay radiatively to the singlet ground state, thereby increasing the overall decay rate. 
Note that MQ is distinct from other quenching mechanisms induced by interactions with external (unpaired) electrons, even though they too involve spin interactions [369]. Annihilation can either occur with an external electron, or the interaction can convert the triplet atom into a singlet state, which then self-annihilates. There are several ways this can occur, namely: (1) triplet-singlet conversion by electron (or positron [288]) exchange (spin exchange quenching (SEQ) $[17,19])$, (2) spin conversion via spinorbit interactions (spin orbit quenching (SOQ) [370]), (3) direct annihilation with an external electron of opposite spin (pick off annihilation (POA) [371]) or, (4) temporary Ps attachment to a molecule, followed by annihilation (chemical quenching (CQ) [372]). Here the term magnetic quenching will be used only for those cases in which Zeeman mixing between singlet and triplet Ps states is induced by an external magnetic field.

The experiments of Ziock and co-workers [104,105] constitute the first experimental observation of MQ via $n=2$ Ps states. In this work both an applied electric field and motional Stark effects were present. The data obtained were not sufficiently accurate to study these effects in detail, primarily because of the single-event counting described above. Using single-shot lifetime methods, however, MQ can be used to detect excited states without photoionization $[109,127,135]$. This can sometimes be convenient since it does not require the use of two synchronized lasers, one of which must usually be very intense [106]. Moreover, MQ allows state mixing processes to be studied, since only selected excited states will be detected, in contrast to photoionization which usually is not state selective in Ps experiments.

The magnetic field strength needed to mix states decreases as the levels get closer, and so has an $n^{-3}$ dependence (see Eq. (5)). Thus, while $\approx 0.1 \mathrm{~T}$ fields are required to significantly mix ground state atoms, fields of the order of $0.01 \mathrm{~T}$ will mix $n=2$ levels; such fields are typical of those used to guide positron beams. Electric fields do not significantly affect ground state atoms (there are no $\ell$ states to mix) but they do affect excited states, and both Stark and Zeeman mixing must be accounted for when excited states are studied (see Fig. 5).

Figure 9 shows the results of experiments in which a UV laser $(\lambda=243.01 \mathrm{~nm})$ was used to excite Ps atoms in a $13 \mathrm{mT}$ magnetic field $B$ with applied parallel electric fields $F$ with strengths up to $\pm 3 \mathrm{kV} \mathrm{cm}^{-1}[129,130]$ (see Fig. 8). The signal parameter $S_{\gamma}$ (see Eq. (45)) was measured using SSPALS for different electric fields, and with different laser polarizations. $S_{\gamma}$ is proportional to the fraction of atoms that are excited by the laser and are quenched, and in this measurement the analysis used was such that a positive (negative) $S_{\gamma}$ value implies that Ps lifetimes have been reduced (increased) relative to the ground state lifetime [357].

The laser light polarization used affects MQ signals because it determines which $n=2$ excited states are accessible under the selection rules for electric dipole transitions from the $M_{J}=0, \pm 1$ sublevels of the $1{ }^{3} \mathrm{~S}_{1}$ state. When the laser radiation is polarized parallel to the $z$-axis (Fig. 9a), $\Delta M_{J}=0$ transitions to the outer triplet Stark states dominate when $F \gtrsim 1 \mathrm{kV} \mathrm{cm}^{-1}$ (see
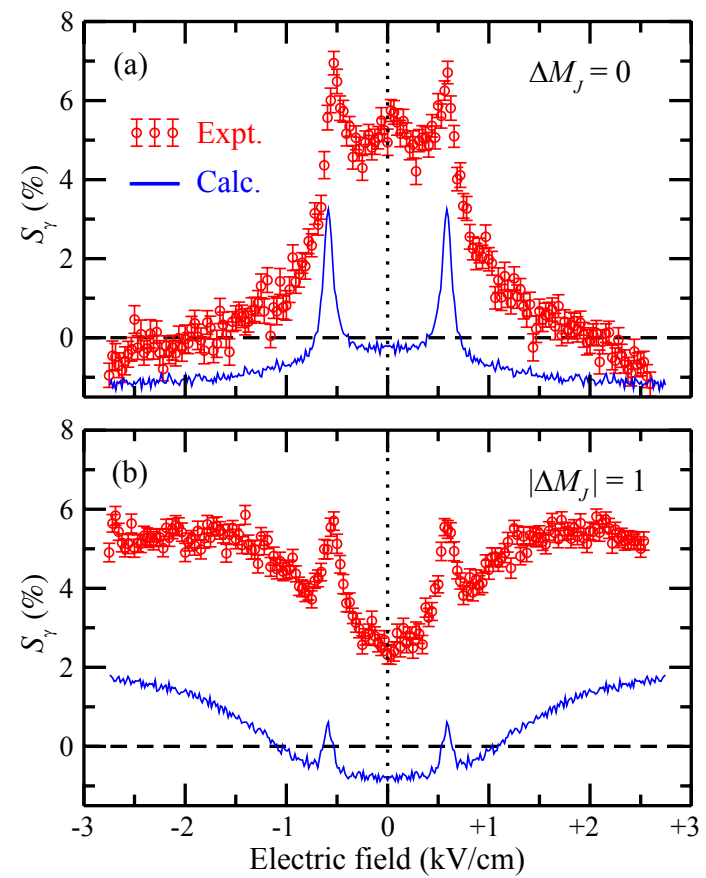

Fig. 9. Dependence of the Ps annihilation signal $S_{\gamma}$ on the electric field for photoexcitation with the laser radiation linearly polarized (a) parallel and (b) perpendicular to the external $13 \mathrm{mT}$ magnetic field. Both measured data (symbols) and the results of Monte Carlo calculations (solid lines) are shown. From reference [130].

Fig. 5). Because of their combined $2{ }^{3} \mathrm{~S}$ and $2{ }^{3} \mathrm{P}$ character, these states may live longer than the $1^{3} \mathrm{~S}_{1}$ state, causing $S_{\gamma}$ to become negative in the highest electric fields. For lower fields (i.e., $F \lesssim 1 \mathrm{kV} \mathrm{cm}^{-1}$ ) coupling to singlet-triplet mixed $2 \mathrm{P}$ terms allows fluorescence to the short-lived $1{ }^{1} \mathrm{~S}_{0}$ state, and hence $S_{\gamma}$ is positive. The peaks at $\sim 585 \mathrm{~V} \mathrm{~cm}^{-1}$, are due to the almost equal singlet and triplet character of the mixed Stark states, as highlighted in Figure 5b. MQ occurs as a combination between excitation via the triplet character of a mixed state, and decay via the singlet character of that same state. The avoided crossing (due to the presence of the magnetic field) in an electric field of $\sim 585 \mathrm{~V} \mathrm{~cm}^{-1}$, optimizes both of these, giving approximately 50\% triplet and 50\% singlet, resulting in optimal MQ.

When the laser light is polarized perpendicular to the $z$-axis, (Fig. 9b), $\Delta M_{J}= \pm 1$ transitions are driven. This results in significant singlet-triplet mixing, an increased annihilation rate, and thus positive $S_{\gamma}$ values. The resonance associated with the transition to the optimally mixed singlet-triplet state at $\sim 585 \mathrm{~V} \mathrm{~cm}^{-1}$ is also accessible in this case [130], and so the MQ peaks are also present.

The expected MQ signal was calculated using a MonteCarlo model. This took into account all allowed decay pathways, namely; fluorescence to $1^{3} \mathrm{~S}_{1}$ or to the $1{ }^{1} \mathrm{~S}_{0}$ levels, and annihilation from $n=2$ due to mixing with $2^{3} \mathrm{~S}_{1}$ or $2{ }^{1} \mathrm{~S}_{0}$ levels. These pathways were sampled using Monte Carlo methods, and all annihilation events used 
to produce simulated decay curves. A peak was added at $t=0$ so as to generate the experimentally measured values of $f_{\mathrm{d}}$ (see Eq. (44)). These spectra were convoluted with the detector time response, and then used to generate simulated $S_{\gamma}$ values, which are shown in Figure 9. Note that these data have not been scaled. The quantitative agreement with the experimental data indicate that general physical mechanisms behind the MQ curves have been correctly identified. The qualitative difference probably means that there are additional mechanisms that have not been included, such as optical pumping effects that alter the different state populations. Whatever their source, the existence of these effects turns out to be serendipitous as it significantly increases the MQ signal, particularly for the $\Delta M_{J}= \pm 1$ transitions.

By producing Stark mixed $n=2$ states it is possible to generate $2{ }^{3} \mathrm{~S}_{1}$ levels using single photon excitation [139]. This is possible because excitation in a strong electric field with the appropriate laser polarization can result in the population of mixed states with significant $2{ }^{3} \mathrm{~S}_{1}$ character (see Fig. 5). If the electric field in which these states are produced is turned off at a rate comparable to the decay rate of the mixed states then it is possible for these states to adiabatically evolve into pure $2{ }^{3} \mathrm{~S}_{1}$ levels. The main difficulty in performing such an experiment lies in controlling the electric fields on the appropriate time scale. This can be done using solid-state high-voltage switches, but one must still pay attention to the time dependences of applied voltages and the resulting electric fields, which may not be changing in a fully controllable manner, and therefore should be checked with additional diagnostics [139].

Figure 10 shows TOF data recorded in different electric fields under conditions in which mixed states with a significant $2{ }^{3} \mathrm{~S}_{1}$ component would be present [139]. When the indicated excitation electric fields were switched off the presence of a longer-lived component indicated that some atoms were left in pure $2{ }^{3} \mathrm{~S}_{1}$ states, which have a lifetime of $1.1 \mu \mathrm{s}$, due to annihilation (see Tab. 2). These TOF data were recorded by a gamma-ray detector located $\approx 30 \mathrm{~cm}$ away from the Ps production target, and which is therefore unable to detect ground state atoms. The increased yield when the field is turned off (i.e., for the case $E=E(t))$ clearly demonstrates $2{ }^{3} \mathrm{~S}_{1}$ production. However, the production efficiency of $0.3 \%$ observed in this experiment was considerably lower than the expected $2 \%$, primarily because of the field switching times. Possible improvements could be obtained by producing a spatially varying field that atoms fly through so as to lower the field they experience as quickly as possible while remaining in the adiabatic regime.

Zeeman mixing in the $n=2$ manifold can also facilitate an optical measurement of the Ps hyperfine interval by making it possible to excite atoms from the triplet ground state to $n=2$ mixed states via a single photon transition, and then optically driving them to the singlet ground state with the same laser [121]. This process is clearly forbidden by electric dipole selection rules, and can occur only via Zeeman-mixed states. The measurement is in fact a type of saturation spectroscopy (SS) [343,373], which is a well-known technique used for performing sub-Doppler

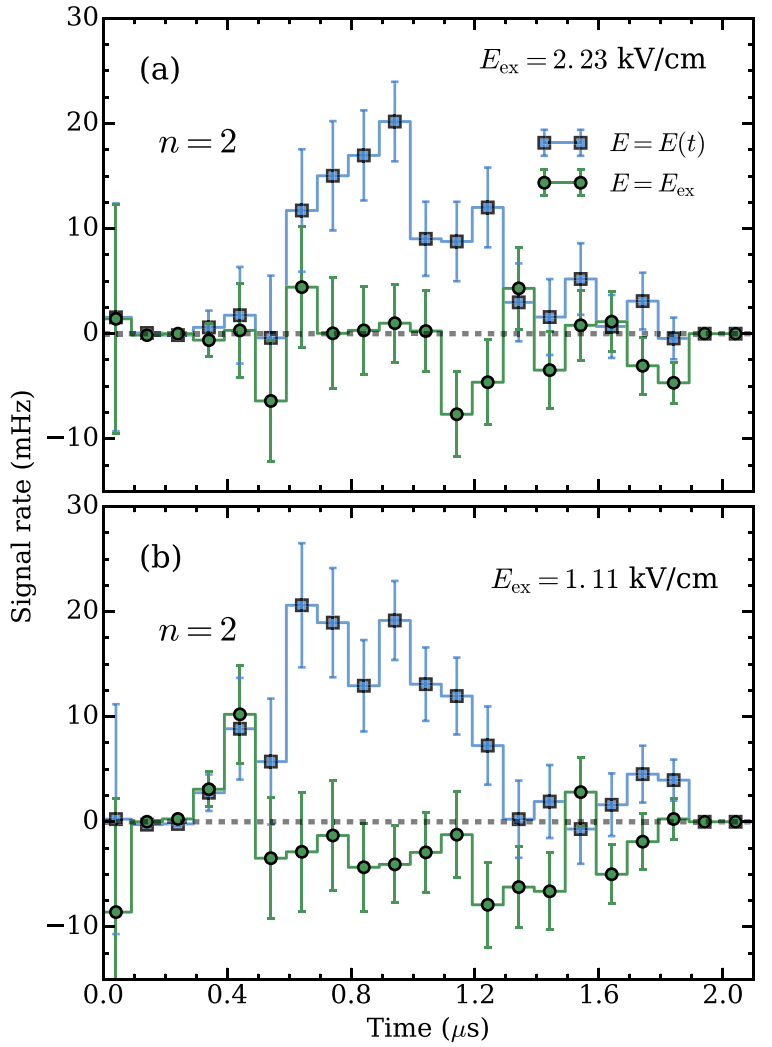

Fig. 10. Background subtracted TOF spectra recorded with lasers tuned to excite $n=2$ states in combined electric and magnetic fields. Data were recorded in different excitation electric fields, which are indicated in each panel. When the fields are switched off (square symbols) an excess of delayed counts are observed, due to the production of long-lived $2{ }^{3} \mathrm{~S}_{1}$ atoms. This signal is not observed if the excitation fields are maintained (circles). From reference [139].

measurements of (single-photon) transitions. Ordinarily SS employs an intense pump beam that saturates the transition being studied, and a weaker counter-propagating probe beam (often derived from the pump beam) that samples the gas without perturbing it, although there are numerous variants of the technique [227]. For narrow laser bandwidths, both the pump and probe beams address zero velocity atoms on resonance, whereas off resonance they address different atoms due to their opposite Doppler shifts. Thus, on resonance the probe beam observes fewer atoms (because they are already in excited states) and the lineshape will exhibit a local minimum, known as a Lamb $\operatorname{dip}[374,375]$.

In order to perform SS with positronium the low number of atoms means that it is not feasible to measure the attenuation of a probe laser. However, one can use the Ps annihilation gamma radiation as a probe if excited states have an increased decay rate, which will be the case if MQ occurs (or if an ionizing laser is present). Then both counter-propagating laser beams are effectively intense pump beams and, just as in standard SS measurements, the signal on resonance will be depleted as both laser 


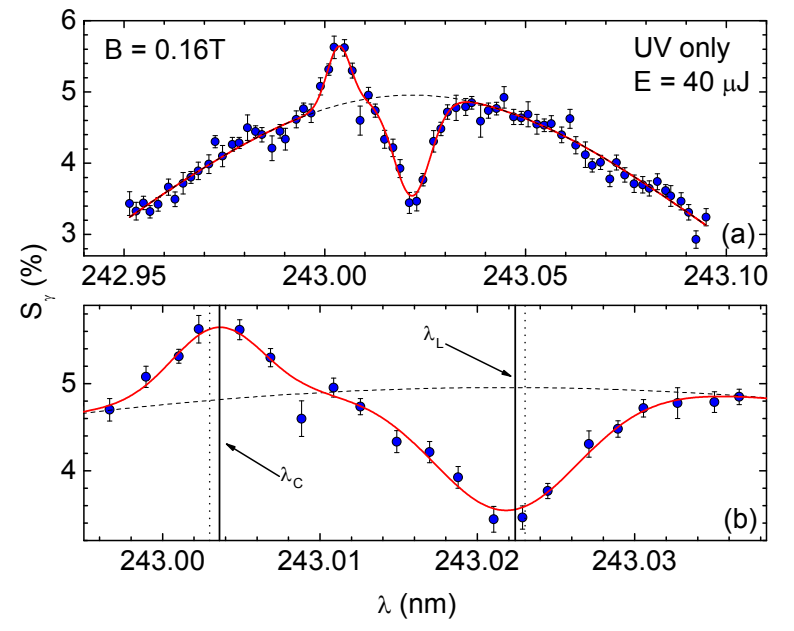

Fig. 11. Saturated spectroscopy measurement of the Ps hyperfine interval. The top panel (a) shows a section of the lineshape with the Lamb dip and crossover peaks. The solid line is a multi-Gaussian fit and the dotted line shows the component of that fit representing only the $1^{3} \mathrm{~S}_{1} \rightarrow 2{ }^{3} \mathrm{P}_{\mathrm{J}}$ transition. The lower panel (b) is an expanded version of (a) indicating the calculated positions of the Lamb dip and hyperfine crossover peak, with (solid vertical) and without (dotted vertical) including Ps recoil. From [121].

beams address the same atoms. The results of such a measurement are shown in Figure 11. In this experiment a $0.16 \mathrm{~T}$ magnetic field was present, ensuring a strong MQ signal even with zero applied electric field (see Fig. 9). Figure 11 shows the central region of a $1{ }^{3} \mathrm{~S}_{1} \rightarrow 2{ }^{3} \mathrm{P}_{\mathrm{J}}$ scan, and a Lamb dip is clearly visible at the resonance frequency, $\lambda_{0}=243.021 \mathrm{~nm}$. The width of this peak is largely determined by the width of the excitation lasers, although the present case also exhibits considerable power broadening, despite the relatively low laser pulse energy of $40 \mu \mathrm{J}$.

A Lamb dip measurement alone cannot yield the hyperfine interval, $\nu_{\mathrm{HFS}}$, as it depends only on the $1^{3} \mathrm{~S}_{1} \rightarrow 2{ }^{3} \mathrm{P}_{\mathrm{J}}$ transition frequency. It is also necessary to measure a cross-over peak [227]. These arise if transitions take place to (or from) states with two common levels that are within the Doppler profile of the laser. Then, if the laser wavelength is tuned to be exactly half way between the two neighboring peaks, some atoms may be red-shifted into resonance with one laser beam (and excited), and be blue-shifted into resonance with the laser traveling in the opposite direction, allowing stimulated emission to take place. In the present measurement the overlapping levels are the triplet and singlet ground states, which are coupled via Zeeman mixed excited states [121]. The cross-over peak is visible in Figure 11, at wavelength $\lambda_{c}=243.003 \mathrm{~nm}$ (i.e., approximately $100 \mathrm{GHz}$ away from the Lamb dip). There is more than one accessible mixed excited state, but in this measurement they cannot be resolved. The power-broadening of the Lamb dip can be clearly seen in this figure; it is roughly half as wide as the shift of the cross-over peak; i.e., it is close to $50 \mathrm{GHz}$ wide, despite the fact that the laser bandwidth used was approximately $15 \mathrm{GHz}$ [121]. Energy and momentum considerations indicate that the frequencies of the Lamb dip $\nu_{\mathrm{L}}$ and the crossover peak $\nu_{\mathrm{C}}$ will be given by

$$
\begin{aligned}
& \nu_{\mathrm{L}}=\nu_{\mathrm{X}}(1+R / 2) \\
& \nu_{\mathrm{C}}=\left(\nu_{\mathrm{X}}+\nu_{\mathrm{HFS}} / 2\right)(1-R / 2),
\end{aligned}
$$

where $\nu_{\mathrm{X}}$ is the frequency of the transition from the triplet ground state to the Zeeman mixed $n=2$ level, and $R=$ $h \nu_{\mathrm{X}} / 2 m_{e} c^{2}$. This latter term is necessary because even at low-levels of precision Ps recoil has to be taken into account. Figure 11b shows the expected positions for the Lamb dip and cross-over peaks, with and without recoil effects. One can use measurements of $\nu_{\mathrm{L}}$ and $\nu_{\mathrm{C}}$ to obtain $\nu_{\mathrm{HFS}}$ since

$$
\nu_{\mathrm{HFS}}=2\left(\nu_{\mathrm{C}}-\nu_{\mathrm{L}}\right)+\left(\nu_{\mathrm{C}}+\nu_{\mathrm{L}}\right) R
$$

The hyperfine interval measurement yields $\nu_{\mathrm{HFS}}=198.4 \pm$ $4.2 \mathrm{GHz}$ [121]: this few percent precision is limited primarily by low statistics, hot Ps, and the relatively broad laser bandwidth. The possibility of improving this measurement so that it becomes competitive with the precision ( $\mathrm{ppm}$ ) microwave measurements $[81,313,322,376,377]$ has been discussed in reference [119]. In principle ppm level measurements are thought to be possible with many months of data collection, but only by implementing several very significant improvements, including utilizing a much more intense positron source. It is questionable, therefore, if this exact methodology would be worth pursuing; alternative optical schemes (e.g., Raman spectroscopy [227]) may prove to be more efficacious.

Another alternative method used to measure the Ps hyperfine interval is via quantum beats in the oscillation of annihilation radiation due to interference between singlet and triplet ground states. This effect has been observed by Baryshevsky and co-workers [378,379], and various other researchers [380-383]. However, this technique is unlikely to be competitive with microwave spectroscopy because it is necessary to know the magnetic field with extremely high precision. However, since $E_{\mathrm{hfs}}$ can be accurately calculated, the methodology can be used to produce a magnetometer.

In very strong magnetic fields (i.e., in the Paschen-Back regime [227]) MQ is suppressed, since in this case Zeeman shifts can be sufficiently large to inhibit mixing between singlet and triplet levels. Measurements of this effect [117] are shown in Figure 12. These data show the quenching of ground state Ps when no lasers are present (see Sect. 2.2.2) and quenching of excited states in lower fields. However, when the field is increased the $n=2$ quenching curve approaches that measured with no laser, because excitation can no longer affect the Ps lifetime in an observable way. For comparison data is also recorded with $532 \mathrm{~nm}$ ionizing laser light present, verifying that excited states are still being produced. These data show that it may be possible to perform Ps laser cooling in a strong magnetic field. As described in Section 5.4, Ps laser cooling experiments [384] using the $1{ }^{3} \mathrm{~S}_{1} \rightarrow 2{ }^{3} \mathrm{P}_{\mathrm{J}}$ transition have been proposed. For cooling to occur there must be spontaneous emission, which means that in principle atoms can be lost 


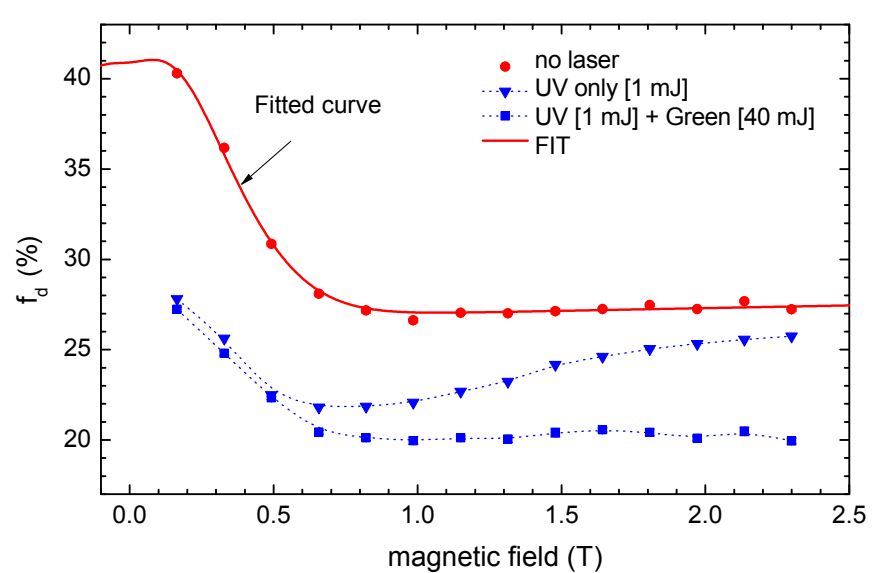

Fig. 12. Suppression of magnetic quenching in a strong magnetic field. The fitted curve for ground state quenching (no laser) is based on equation (31). Here a reduction in the delayed fraction $f_{d}$ (see Eq. (44)) indicates MQ. The approximately constant difference between the ground state and ionization $(\mathrm{UV}+$ Green) curves demonstrates that the production and ionization of $n=2$ excited states is essentially unaffected by the increasing field strength. Conversely, the UV only curve approaches the ground state curve as MQ is suppressed in the increasing field. From [117].

via MQ effects. However, this can be completely avoided if no magnetic field is present, or if a very strong $(\approx 3 \mathrm{~T})$ magnetic field is applied. Conversely, in intermediate magnetic fields, if the laser polarization and electric field are appropriately selected, mixing between singlet and triplet levels may be inhibited, and laser cooling would then, in principle, again be possible (see Sect. 5.4 for a discussion regarding the likely efficacy Ps laser cooling).

\subsubsection{Doppler and time-of-flight spectroscopy}

As discussed previously, single-photon laser excitation of Ps is made more difficult by the very large Doppler broadening that attends the fast Ps produced by essentially all useful Ps sources. This does mean, however, that it is relatively easy to measure Ps energies via the Dopplerbroadened width of $1{ }^{3} \mathrm{~S}_{1} \rightarrow 2{ }^{3} \mathrm{P}_{\mathrm{J}}$ transitions. This can be done in different geometries to obtain the Ps energies perpendicular or parallel to the surface of Ps converters $[109,113]$. Such measurements can be used, with appropriate additional parameters, such as laser delays, to obtain Ps thermalization and emission rates [110]. An example of Ps Doppler profiles measured using a mesoporous silica film converter is shown in Figure 13 [109]. These data show Ps cooling as the positron beam energy is increased, an effect also observed using TOF methods [247].

Measurements of Ps cooling rates in mesoporous thin films, aerogels, and various powders has previously been accomplished via measurements of annihilation radiation, using either Doppler broadening or angular correlation methods [65]. Angular Correlation of Annihilation Radiation (ACAR) is a technique that uses the angle between the photons emitted in a two-photon decay to determine the momentum of the annihilating electron-positron pair with very high resolution (e.g. [385]). This is achieved by

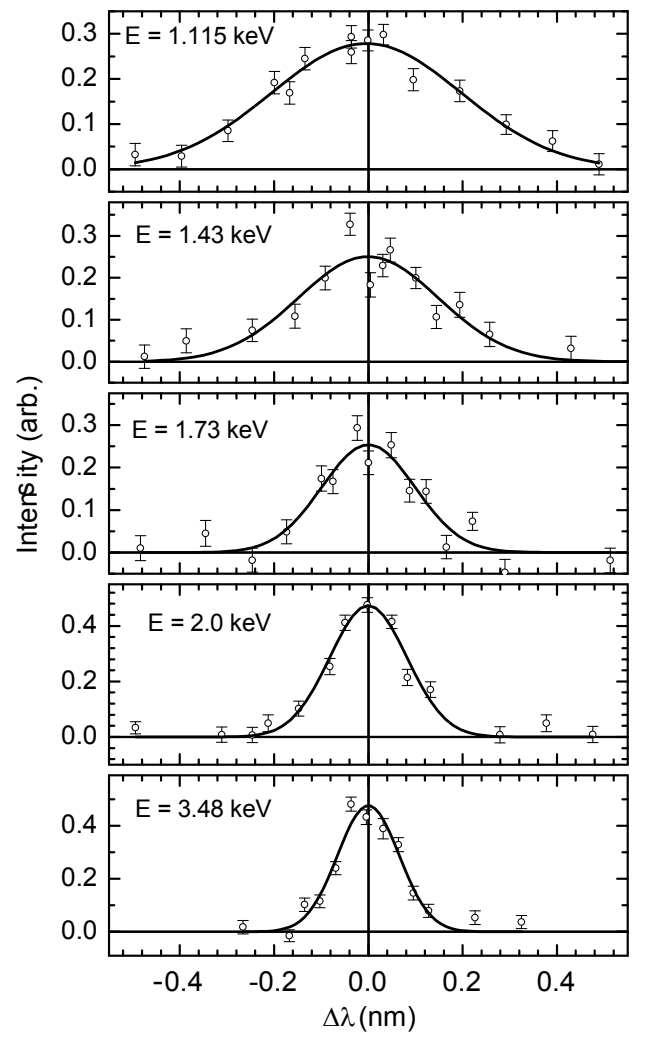

Fig. 13. $1^{3} \mathrm{~S}_{1} \rightarrow 2{ }^{3} \mathrm{P}_{\mathrm{J}}$ resonances measured for Ps produced from a mesoporous silica film with different incident positron beam implantation energies, as indicated in the panels. The data are shown in terms of the laser detuning from the measured resonance wavelength $(\approx 243.02 \mathrm{~nm})$. The positron beam energy-dependent linewidths occur because the resultant Ps energy depends on the positron beam implantation energy. From reference [109].

using position-sensitive gamma-ray detectors (known as Anger cameras, after their inventor, Hal Anger [386]) that are located far away (many meters) from the annihilation events to achieve a high angular resolution. This comes at the cost of the solid angle, however, and the count rates in such measurements are therefore invariably low [387]. In order to obtain cooling rates using this technique, the ACAR data must be time selected (TSACAR), which further reduces the data acquisition rate $[239,294,388]$.

Ps energies can also be measured using two-photon annihilation radiation via Doppler Broadening Spectroscopy (DBS) of the $511 \mathrm{keV}$ photopeak. This technique is limited by the energy resolution of $\gamma$-ray detectors, which for high purity Ge (HPGe) detectors, the current state-of-the-art, is typically a few $\%$ at $511 \mathrm{keV}$. This corresponds directly to Ps energies of a few eV. However, the intrinsic energy resolution of the technique is much better than this; it is possible to deconvolve the annihilation radiation spectra and infer Ps energies on the meV scale, in much the same way as one might split an atomic resonance lineshape (e.g. [389]). As with ACAR, cooling rates may be obtained from DBS measurements if the data are time-selected [390,391], a technique sometimes referred to as Age-Momentum Correlation, or AMOC $[80,392,393]$. 


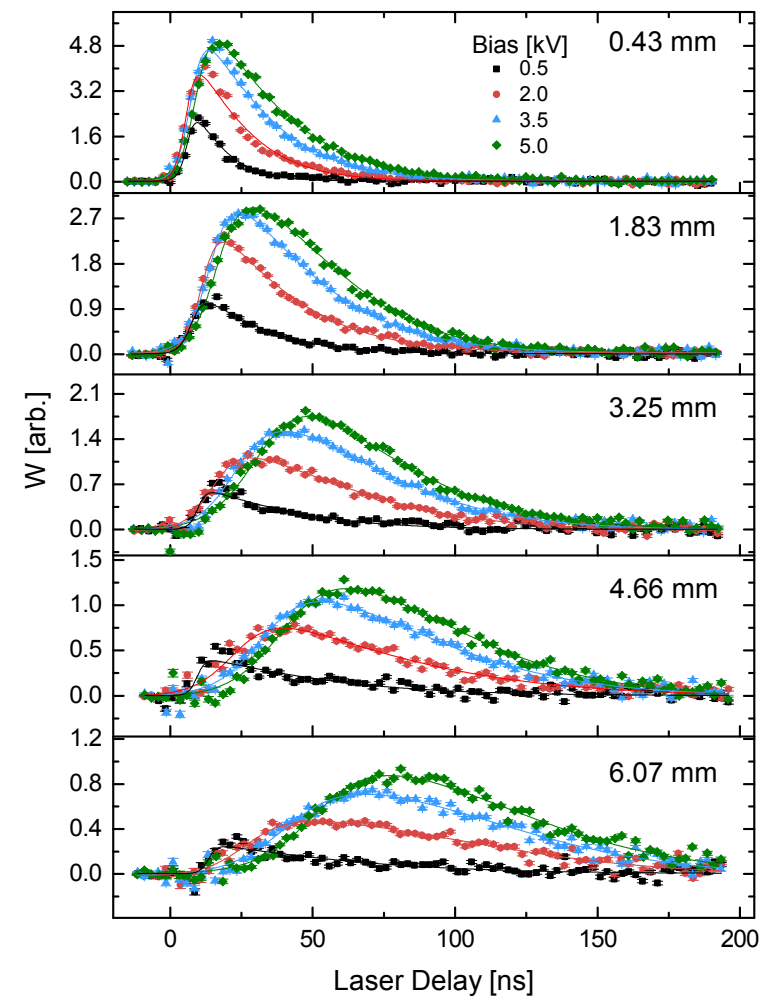

Fig. 14. Laser enhanced Ps time of flight spectroscopy. The parameter $W$ is a measure of the total count rate, as measured via appropriate integration of SSPALS spectra. The TOF profiles were recorded with the excitation laser centered at different longitudinal distances from the Ps source, and at different positron beam implantation energies, as indicated in the figure. From reference [126].

A recent advance in this area takes advantage of the fact that, during o-Ps-Xe collisions, a spin conversion process can occur via spin-orbit interactions, resulting in a twophoton decay $[370,394]$. This interaction occurs via p-wave scattering and is therefore highly energy dependent, which means that measurements of the two-photon annihilation rate can be used to measure the Ps energy as a function of time with high sensitivity $[395,396]$.

Both ACAR and DBS (and variants thereof) require two-photon decays, since three-photon annihilation photons have a distribution of energies and emission angles (see Sect. 2.1.2). These methods therefore only work in situations where Ps atoms can decay via two photons. For measurements made in strong magnetic fields o-Ps cooling rates can be obtained from two-photon decays since the $m=0$ triplets will mix with $m=0$ singlets and have some probability of decaying into two photons (see Sect. 2.2.2).

TOF methods can be used to measure the longitudinal energy of free Ps atoms in vacuum $[52,163,237,238,257]$. The standard way of performing such measurements is to use highly collimated $\gamma$-ray detectors that can observe spontaneous Ps decays with high spatial resolution at a known longitudinal distance from the Ps source. By measuring the Ps decay rate as a function of distance from the source TOF spectra may be generated. Since Ps vacuum decay rates are well-known, such data can be used to determine the Ps velocity, and hence energy, distribution along the direction of travel. This is useful in studies of Ps formation as well as materials science experiments, since Ps emission profiles may contain information about the electronic structure of the surface from which the atoms are emitted [234] (see also Sect. 3.2).

One limitation of this technique is that only those Ps atoms that happen to decay in the field of view of the detector(s) can contribute to the signal. Thus, even if multiple detectors are employed, count rates are low and the Ps atoms are not used efficiently. This problem can be avoided by using lasers to excite Ps atoms at any desired location, and with arbitrarily high spatial resolution. Excited atoms may then be photo-ionized, or may decay via MQ, and the $\gamma$-ray detectors arranged to have maximal solid angle coverage for all space, significantly increasing the available signal [126]. Examples of TOF spectra obtained in this way are shown in Figure 14. These data were recorded using a Ps converter similar to that used to obtain the Doppler profiles of Figure 13. In both cases the same positron beam energy dependent Ps energies are evident; the Doppler and TOF spectra measure the (correlated) transverse and longitudinal Ps energies, respectively [126]. For laser enhanced TOF measurements one must take into account the fact that the laser excitation imposes some velocity selection, and the full Ps distribution is not sampled unless one also scans the excitation laser wavelength. The $\approx 100 \mathrm{GHz}$ bandwidth typically employed to drive $1{ }^{3} \mathrm{~S}_{1} \rightarrow 2{ }^{3} \mathrm{P}_{\mathrm{J}}$ transitions corresponds to a velocity spread of only $0.4 \mathrm{meV}$ (along the direction of the laser beam).

\subsubsection{Spectroscopy of confined Ps}

As discussed in Section 2.2.1, mesoporous silica films can be used to produce Ps atoms, and also to facilitate PsPs interactions. These useful properties are partly due to the dynamics of Ps in such structures, which are therefore interesting areas of study. The effects of confinement on atoms have long been studied as they are relevant to many physical systems, such as many-body physics of atoms in clusters, the behavior of atoms in high pressure environments, and physically confined atoms in buckyballs or other hard-wall potentials [397-402]. Ps is a unique system for such studies [403]: spectroscopy of "normal" atoms can be used (e.g. [404,405]), but Ps has some advantages, namely: (1) the atoms can be produced inside isolated voids following positron bombardment, (2) Ps annihilation radiation can be used as a signal and is easily detected outside the material being studied, and (3) Ps atoms are light, and so may have a de Broglie wavelength comparable to the internal spaces being investigated. This leads to stronger interactions with the walls, and may therefore be more useful for studying the dynamics of interactions with the confining surfaces.

When Ps de Broglie wavelengths become comparable to the length scale of a confining structure, movement within that structure will be determined by tunneling, and be much slower [290]. The modified Ps dynamics resulting from such quantum confinement [290] can be advantageous in studies of Ps-Ps interactions [287,288,362] 


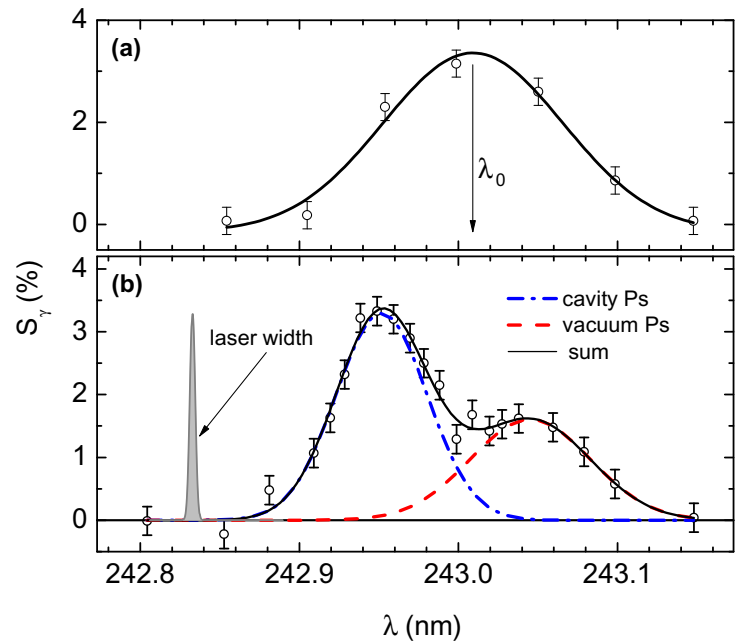

Fig. 15. Observed shift and narrowing of the $1^{3} \mathrm{~S}_{1}-2^{3} \mathrm{P}_{\mathrm{J}}$ transition inside a mesoporous network. Ps excitation was driven in vacuum (a) and inside the pore structure (b) by directing lasers into the target. The red and blue-shifted peaks are attributed to Ps atoms in vacuum moving towards the laser, and the effects of confinement, respectively. Time delayed lineshapes of this type can be used to determine the time taken for Ps to be emitted into vacuum. From reference [115].

because it restricts the available volume that can be sampled, increasing the effective Ps density. Ps created in mesoporous voids with a high enough density to allow PsPs interactions to occur [287] can reveal information about the confining medium via changes in the Ps-Ps interaction rate. The effects of confinement on single atoms, however, can be studied via laser spectroscopy. In particular, $1^{3} \mathrm{~S}_{1}-$ $2^{3} \mathrm{P}_{\mathrm{J}}$ transitions can be observed in Ps atoms that are confined in mesoporous voids. The confinement leads to a shift of the Ps resonance frequency that is related to the pore size, as well as interactions with internal surfaces. Confinement also means that the $\mathrm{Ps}_{\mathrm{s}}$ atom velocities may be zero (on average) on the time scale of a Rabi oscillation. For example, thermal $\mathrm{Ps}$ in a $5 \mathrm{~nm}$ void will have a bounce frequency of more than $10^{12} \mathrm{~Hz}$. As a result we would expect to see a significant narrowing of the linewidth [406].

A shift and narrowing of the $1^{3} \mathrm{~S}_{1}-2^{3} \mathrm{P}_{\mathrm{J}}$ transition have been observed in experiments using mesoporous samples with pore diameters of $\approx 5 \mathrm{~nm}$ [115], as shown in Figure 15 . These experiments were performed by directing a laser either parallel to a mesoporous silica film surface, or into the film when it was rotated by $45^{\circ}$ relative to the positron beam axis. In the former case the usual Ps vacuum resonance line was observed. In the latter case two peaks were observed; one red-shifted peak was attributed the excitation of vacuum Ps moving on average towards the laser beam. A second, blue-shifted, peak was also observed, which was attributed to Ps excited while still in the porous structure. This shift arises from the effect of confinement on the excited state Ps energy levels: in effect squeezing the atom increases the $1^{3} \mathrm{~S}_{1}-2^{3} \mathrm{P}_{\mathrm{J}}$ energy interval [403].

The cavity Ps did exhibit some degree of line narrowing, in that the inferred linewidth was narrower than the vacuum $\mathrm{Ps}$ lineshape, and would represent sub-thermal
Ps if it were due to Doppler broadening. However, Dicke line narrowing would be expected to produce significantly narrower linewidths. The confined Ps also exhibited a significant shift, generally consistent with expectations $[115,403]$. However, because the pore structure comprises many interconnected voids, there is likely no singlevalued shift. One interpretation of the observed data is then that it actually consists of many line-narrowed peaks, all shifted by different amounts, resulting in an approximately Gaussian lineshape with a width that is representative of the effective distribution of pore sizes and the geometry of the interconnects.

The cavity shift is non-trivial to calculate [403,407], even for an idealized material. Assuming hard-wall potentials we can, however, make an order of magnitude estimate simply by considering the size of the ground and excited state atoms. The confinement energy $E_{0}$ of $\mathrm{Ps}_{\mathrm{s}}$ in an infinite spherical potential is (see Eq. (23)) $750 \mathrm{meV} / a^{2}$, where $a$ is the cavity diameter in nm. Thus, in $5 \mathrm{~nm}$ pores $E_{0} \approx 30 \mathrm{meV}$. If we assume that the ground and excited state Ps atoms have radii of 1 and $4 a_{0}$ respectively and we take the effective reduction of the cavity size to be the increased size of the excited state, we find an energy shift of $\approx 4 \mathrm{meV}$ [115]. A detailed analysis of confined Ps has been made by Brown and co-workers who find an energy shift given by

$$
\Delta E \approx \frac{\pi^{2}}{2 R^{3}}\left(\rho_{2 \mathrm{P}}-\rho_{1 \mathrm{~s}}\right)
$$

where $\rho_{1 \mathrm{~S}}$ and $\rho_{2 \mathrm{P}}$ are the static radii of the $1 S$ and $2 P$ states respectively, and atomic units are used. These authors find $\rho_{1 \mathrm{~S}}=1.65 a_{0}$ and $\rho_{2 \mathrm{P}}=5.35 a_{0}$. Using $R=$ $50 a_{0}$ they also obtain an energy shift of $\approx 4 \mathrm{meV}$; both estimates disagree with the measured shift of $1.3 \mathrm{meV}$ [115]. This may be related to the specific properties of the target used. The assumption of a $5 \mathrm{~nm}$ pore size cannot be accurate since the pores are all interconnected, and there must therefore exist a continuum of different mean free paths experienced by the Ps. Brown and co-workers [403] speculate that this disagreement may imply that the radius of confined $n=2 \mathrm{Ps}$ is not much larger than that of the ground state atoms, owing to the nature of $\mathrm{Ps}_{\mathrm{S}-\text { wall }}$ interactions.

Preliminary experiments designed to study confined PS have been carried out at UCL using porous films similar to those described above, but made with lower porosity and larger pores [408]. These films have been shown to produce Ps with low efficiency [284], and with very little escaping into vacuum below a porosity threshold of $\approx 60 \%$. Figure 16 shows data obtained using a sample with porosity $P=40 \%$ and a pore size of $32 \mathrm{~nm}$. For this porosity we expect Ps to be localized inside the material [284], where it may be probed with lasers for as long as it lives, as shown in Figure 16b.

The lifetime of confined Ps can be obtained directly from the SSPALS spectra, or from the time dependence of the laser excitation signal. In the former case the lifetime spectra must be deconvoluted from the an instrument response function, which can be measured under conditions when no Ps is generated [287]. The dashed line in 


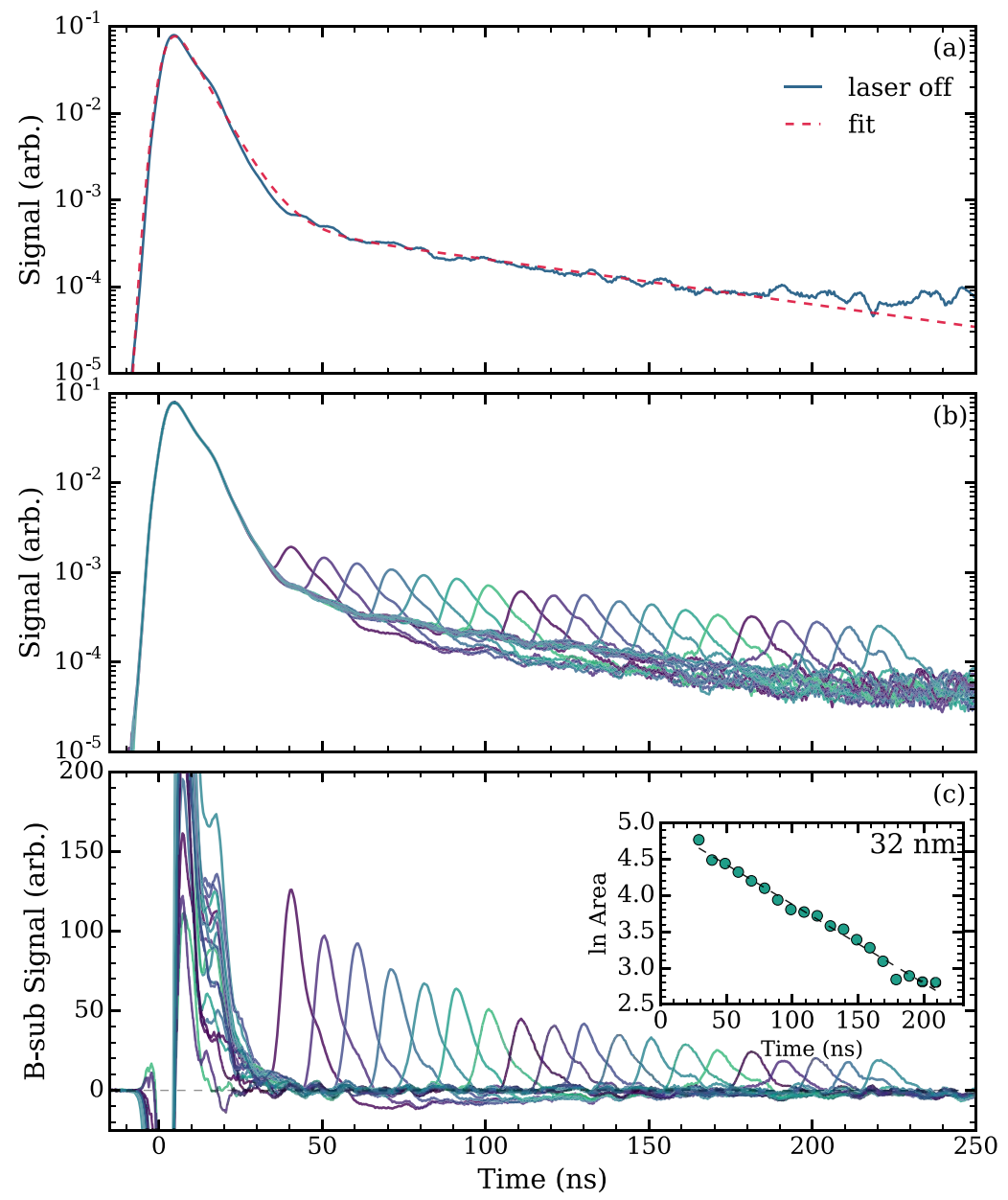

Fig. 16. Lifetime spectra measured with a large diameter $(32 \mathrm{~nm}$ ) isolated pore sample showing (a) a spectrum and fit (see text) with no laser, (b) spectra with the UV laser fired at 10 ns intervals and (c) the background subtracted data from (b). The time dependence of the excess annihilation peaks are shown in the inset to (c) along with a fit.

Figure 16a shows a fit obtained in this way. The laser excitation signal is proportional to the number of atoms remaining and can therefore be used to generate a lifetime spectrum, as indicated in Figure 16c. Both methods yield lifetimes of $\approx 90$ ns.

Although it is interesting to observe confined Ps, for such large pores we would expect a cavity shift roughly $(5 / 32)^{3}$ smaller than that of the $5 \mathrm{~nm}$ samples [403]. This would correspond to a wavelength shift of $0.00025 \mathrm{~nm}$, which would not be observable in our experiments. This laser induced signal in Figure 16b was obtained without using an ionizing laser, and under conditions where MQ (see Sect. 3.1.1) is negligible. However, excitation of the $1^{3} \mathrm{~S}_{1}-2^{3} \mathrm{P}_{\mathrm{J}}$ transition led to immediate annihilation, as is evident from the data. This is in contrast to previous measurements of confined Ps [115]. Furthermore, a very broad excitation lineshape was observed, as shown in Figure 17. The underlying cause of this extreme broadening is not yet understood: one possibility is that isolated pores may contain contaminants that are removed from internal voids connected to the vacuum, and which may lead to chemical quenching [369]. The ground state lifetimes in the pores should be close to the vacuum lifetime
[248,409], which implies that there is a weaker quenching effect affecting ground state atoms, and a much stronger one affecting excited state atoms. If this sort of contamination is common to isolated pore structures then it could have implications for porosimitry and laser cooling experiments. In the former case, ground state lifetimes cannot be reliably predicted by Tao-Eldrup style models [410]. In the latter case, even if slightly reduced ground state lifetimes are acceptable, laser excitation will result in immediate Ps annihilation, and thus laser cooling would not be possible.

\subsection{Excitation of Rydberg states}

The term "Rydberg atom" refers to an atom in an excited state with a high principal quantum number $n$ [175]. What constitutes "high" is a matter of perspective. All atoms and molecules exhibit some version of the basic Bohr energy level structure (see Eq. (1)) and therefore possess Rydberg states. Positronium is no exception to this, and in fact might be considered to be the purest Rydberg system there is, since it is hydrogenic and composed only of leptons, which are thought to be point particles with no internal structure. 


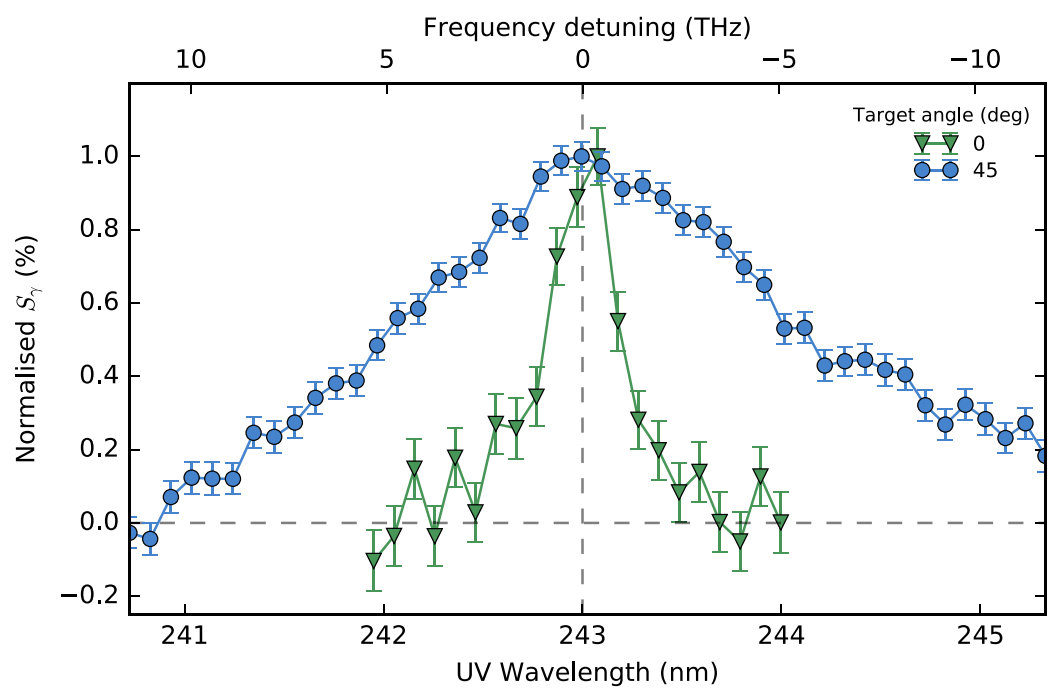

Fig. 17. $1^{3} \mathrm{~S}_{1}-2^{3} \mathrm{P}_{\mathrm{J}}$ excitation lineshapes measured for vacuum and confined Ps. The very broad linewidth observed for confined Ps is thought to be due to Ps interactions with (unidentified) chemical contaminants in the isolated pores.

Table 4. The $n$-dependence of some properties of Rydberg atoms, with examples shown for the $30 d$ state of Ps, H and He. The state separation is calculated for $30 d \rightarrow 31 d$. The orbital radius is defined here as the expectation value

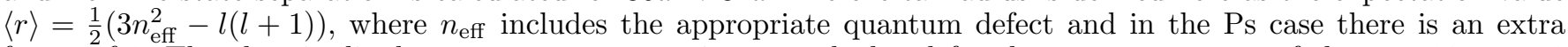
factor of 2. The electric dipole moment-to-mass ratios are calculated for the outermost state of the $n=30, m=2$ Stark manifold. The stated radiative lifetime $n$-dependence applies only to low $\ell$ states: for circular states the scaling is closer to $n^{5}$ (see Fig. 2). From reference [411].

\begin{tabular}{lllll}
\hline & $n$-scaling & Ps & H & He \\
\hline Binding energy $(\mathrm{meV})$ & $n^{-2}$ & -7.56 & -15.11 & -15.12 \\
State separation $(\mathrm{meV})$ & $n^{-3}$ & 0.48 & 0.96 & 0.96 \\
Orbital radius $\left(a_{0}\right)$ & $n^{2}$ & 2694 & 1347 & 1347 \\
Radiative lifetime $(\mu \mathrm{s})$ & $n^{3}$ & 28.4 & 14.2 & 14.2 \\
Dipole moment/mass $\left(e a_{0} / \mathrm{amu}\right)$ & $n^{2}$ & $2.2 \times 10^{6}$ & 1206 & 304 \\
\hline
\end{tabular}

Rydberg atoms and molecules in general have exaggerated properties compared to their ground state counterparts: they may have larger dipole moments, are more polarizable, live longer, and so on. The reduced mass of Ps is exactly $m_{e} / 2$ (exact to the extent that electrons and positrons have the same mass), whereas the reduced mass of $\mathrm{H}$ is $0.9995 m_{e}$. As a result, many of the properties of Rydberg Ps atoms are (almost) a factor of two different from those of the corresponding states in hydrogen; for example, florescence lifetimes in Ps are twice as long as those of H [148]. Some of the properties of $n=30$ Rydberg states of Ps, $\mathrm{H}$ and $\mathrm{He}$ are compared in Table 4. Note that while many Ps Rydberg properties do indeed scale from those of $\mathrm{H}$ by a factor of $\approx 2$, the dipole moment to mass ratio depends also on the electron/proton mass ratio, meaning that Ps should be very easy to control using Stark deceleration methods (see also [412]).

As discussed in Section 3.1, the optical excitation of Ps Rydberg atoms was first accomplished by Ziock and co-workers in 1991 [105] using the two-color two-step excitation process $1{ }^{3} \mathrm{~S} \rightarrow 2^{3} \mathrm{P} \rightarrow n^{3} \mathrm{~S} / n^{3} \mathrm{D}$. It is also possible to produce Rydberg Ps atoms by positron collisions with certain untreated metallic surfaces [413-415]. However, there is no way to control this process, which is in any case extremely inefficient. Another demonstrated way to produce Rydberg Ps is through a charge exchange process in which Rydberg Cs atoms interact with trapped positrons to produce Rydberg Ps. This method was used to make antihydrogen $[416,417]$ via the subsequent interaction of Rydberg Ps with trapped antiprotons. This process is expected to have a very large cross section [418] that scales with $n^{4}$, although this scaling may be suppressed for $n \geq 4$ [419]. The initial Cs atom state can be precisely controlled with cw excitation lasers, allowing full control over the subsequent Ps and antihydrogen formation mechanisms. In addition to its utility in antihydrogen production, this method may also be useful for studies of Rydberg Ps itself. It has the advantage that atoms can be produced in a cw mode, which could be useful for spectroscopic measurements (see Sect. 5.2).

It is in principal possible to excite Ps directly from the ground state to a Rydberg level using a Doppler-free two-photon transition. Attempts to do so at UCL have been unsuccessful, primarily because a narrow-band laser is required so that the laser power is not wasted (much as with the two-photon $1{ }^{3} \mathrm{~S}_{1} \rightarrow 2{ }^{3} \mathrm{~S}_{1}$ case, see Sect. 3.3). However, test experiments using Rydberg He atoms, for which the transition strength is expected to be similar to 
that of Ps excitation, have indicated that this approach is feasible [420].

Pulsed positron beams enable direct Ps excitation using the $1^{3} \mathrm{~S} \rightarrow 2^{3} \mathrm{P} \rightarrow n^{3} \mathrm{~S} / n^{3} \mathrm{D}$ excitation scheme previously demonstrated [105]. However, pure S or D states are not generally produced in experiments; hydrogenic atoms in states with the same $n$ but different $\ell$ values are degenerate unless external fields are present, and electric and magnetic fields are invariably present in Ps experiments (especially if traps are used). Even very small fields can lead to complete $\ell$ mixing within an $n$ manifold [176]. For example, the motional electric fields experienced by Ps moving in the Earth's magnetic field is sufficient to mix all $\ell$ states (e.g. [132]). Thus, while light fields do couple ground state atoms to $\mathrm{S}$ and D states according to electric dipole selection rules, the final states produced will usually be $\ell$-mixed Stark states.

Recent experiments carried out at UCL use a two-stage surko trap to produce pulses containing $\approx 10^{5}$ positrons, compressed to a time with $\Delta t \approx 4 \mathrm{~ns}$ [357]. These are implanted into a mesoporous silica film [284] in order to generate a dilute Ps gas in vacuum. The Ps atoms are optically excited in the same way as in the production of $n=2$ states, but with the addition of a second laser. As in previous work an ultra-violet (UV) laser $(\approx 500 \mu \mathrm{J}$, $\Delta \nu=85 \mathrm{GHz}, \lambda=243.0 \mathrm{~nm})$ drives $1^{3} \mathrm{~S} \rightarrow 2^{3} \mathrm{P}$ transitions. For Rydberg production an infra-red (IR) laser $(\approx 8 \mathrm{~mJ}, \Delta \nu=5 \mathrm{GHz}, \lambda \approx 750 \mathrm{~nm})$ is used to drive $2^{3} \mathrm{P} \rightarrow$ $n^{3} \mathrm{~S} / n^{3} \mathrm{D}$ transitions. This excitation process couples the ground state atoms to the $\mathrm{S}$ or D character of excited Stark states, whose azimuthal quantum number can be controlled via the UV and IR laser light polarizations $[125,129]$.

The experimental requirements necessary to produce Rydberg Ps atoms using optical methods are essentially the same as those required to produce any excited states. The efficiency with which Ps atoms can be transferred from $n=2$ to Rydberg levels has been observed ot be very high $(\geq 90 \%)[118]$. This was attributed to mixing of the Rydberg levels that inhibits stimulated emission back to $n=2$, thereby locking the atoms in the excited states. The precise mechanism has not been unambiguously identified, but subsequent measurements seem also to occur with high efficiency [125]. Since the 2012 demonstration [118] several groups have now produced Rydberg Ps (see Tab. 1) using essentially the same methods (in one case [135] using $n=3$ as the intermediate step, for some reason).

The SSPALS technique described in Section 3.1 can also be used to study Rydberg Ps atoms [118,125,135]. In this case, however, the detector timing requirements are far less stringent; instead of events happening on the $142 \mathrm{~ns}$ time-scale of the $1^{3} \mathrm{~S}_{1}$ lifetime (or faster), long-lived Rydbergs decay on much longer time scales. Rydberg Ps atoms can be detected either following radiative decay and subsequent annihilation, or after collisions with material objects in the vacuum system. An example of single-shot lifetime spectra recorded with $n=8$ Ps atoms is shown in Figure 18. In this case the Ps is detected following both fluorescence decay and annihilation (the radiative lifetime is approximately $1 \mu \mathrm{s}$ ), and chamber wall collisions

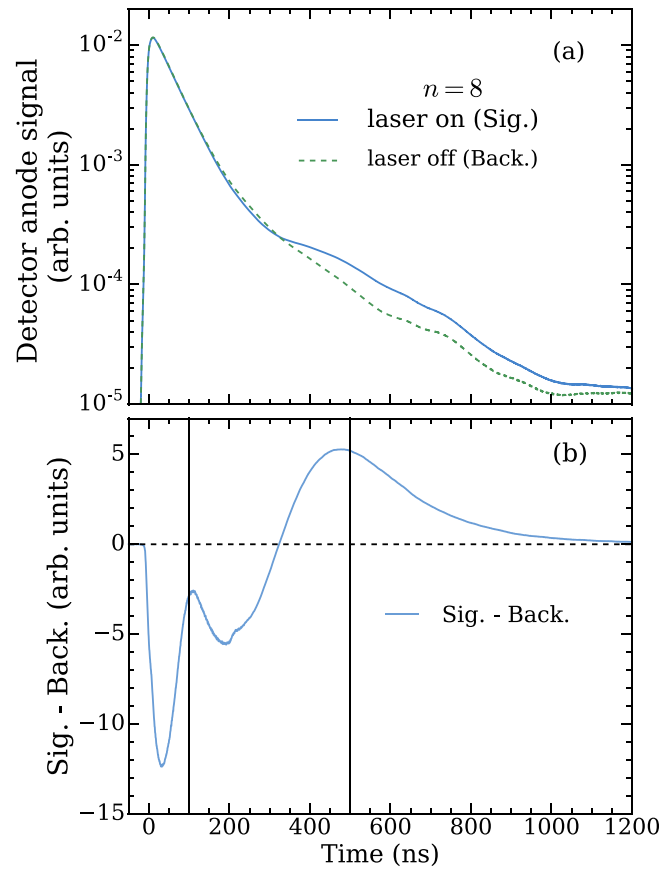

Fig. 18. Example of $n=8$ Rydberg Ps lifetime spectra recorded using a LYSO detector with and without IR laser light present. The difference between the laser on and off curves of (a) are shown in (b). The vertical lines at 100 and $500 \mathrm{~ns}$ indicate the approximate times of Ps annihilations occurring following collisions with the grid electrode and the chamber walls, respectively. The data comprise 29000 pairs of shots and were acquired in $17 \mathrm{~h}$. From reference [139].

$\approx 500 \mathrm{~ns}$ after excitation. For long-lived atoms the PWO detectors originally developed for studying high-intensity Ps pulses with short time scales [359] are less efficacious than slower, but brighter LYSO-based detectors [328].

An example of the excitation of Rydberg levels ranging from $n=9$ to the ionization limit is shown in Figure 19. For these data the parameter $S_{\gamma}$ was measured using lifetime spectra similar to those shown in Figure 18. The falling background level at the longest wavelengths is due to amplified spontaneous emission (ASE) [227] occurring in the IR dye laser, as the wavelength is near the edge of the gain curve for the dye used (Styryl-8). The reversal of the amplitude of the signal is due to field ionization of the higher $n$ states as they pass through the electrode structure used to define the electric field in the target region. States above $n=28$ are not resolved due to the laser bandwidth and Doppler broadening.

The data shown in Figure 19 were recorded in an experimental configuration very similar to that shown in Figure 8, with a slightly different spacing between the electrodes. Here Ps atoms take roughly $100 \mathrm{~ns}$ to reach the grid electrode, as can be seen in Figure 18. After the Rydberg atoms pass through the grid they will experience an electric field (see Fig. 8b) and may therefore be field ionized at a rate that depends on their state and the field present (see Fig. 6). Lifetime spectra are analyzed using appropriate time windows to generate the parameter $S_{\gamma}$ 


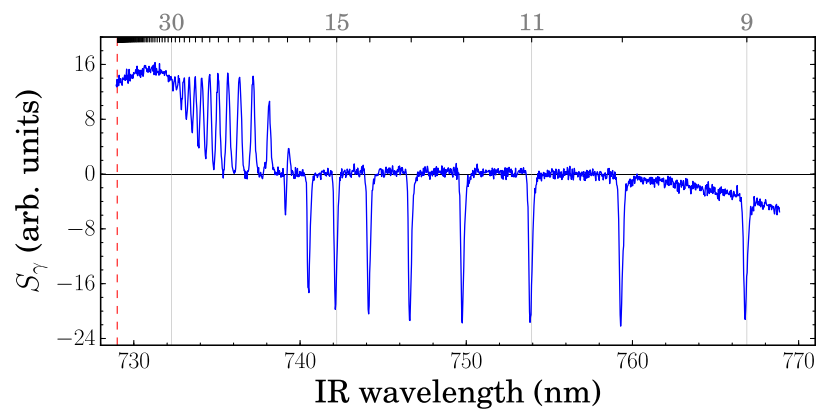

Fig. 19. Measured Ps Rydberg-Stark state lifetimes recorded with a PWO detector. The falling background signal at low $n$ is due to amplified spontaneous emission (ASE) in the IR dye laser, and the reversal of the signal sign is due to the field ionization of atoms with $n \geq 17$ upon leaving the zero-field interaction region. The vertical dashed line indicates the Ps ionization threshold. From reference [125].

(see Eq. (45)). The time windows are optimized for a certain process, such as decays at later times. If Ps decay occurs at early times the sign of $S_{\gamma}$ may then change, as is the case in Figure 19; in general when using SSPALS to observe specific events one adjusts the time windows accordingly (see Eq. (44)). For measurements that encompass different time regions one must take care to analyze the data in the correct way so as to optimize the desired signal. In this particular case the shift from late to early decays was sufficiently far from the edges of the timewindows used to generate a near-optimal signal in both cases (albeit reversed in sign) but this will not be true in general.

It can be seen that the $n=17$ line in Figure 19 is split, with some parts giving a positive $S_{\gamma}$ signal and others a negative $S_{\gamma}$ signal. This indicates that some of the Stark states decay late, while others decay early. Thus we can interpret the split line as a cutoff in transmission through the grid electrode, with those atoms not transmitted annihilating. Since the outermost Stark states (i.e., those with negative Stark shifts) ionize in weaker electric fields than states with positive Stark shifts (see Fig. 6), the cut-off in transmission through the grid occurs first for the those components. This mechanism was studied in more detail for the $n=18$ line (since it is then easier to vary the fields and control the Stark state transmission). The results are shown in Figure 20. These data were recorded with a constant electric field of $63 \mathrm{~V} \mathrm{~cm}^{-1}$ applied to the excitation region, and fields ranging from 1397 to $1985 \mathrm{~V} \mathrm{~cm}^{-1}$ in the region after the grid. For weaker electric fields, the short wavelength components of the $n=18$ spectral feature are transmitted through the grid (negative $S_{\gamma}$ parameter) while only the outermost components at the long wavelength edge are not transmitted. This indicates that Rydberg Stark states are polarized by the electric field in the excitation region, resulting in a partial splitting of states with positive and negative Stark shifts, and that these states then evolve adiabatically while passing from the excitation region through the grid. One can, therefore, filter Stark states with electric fields, even if it is not possible to spectrally resolve them.

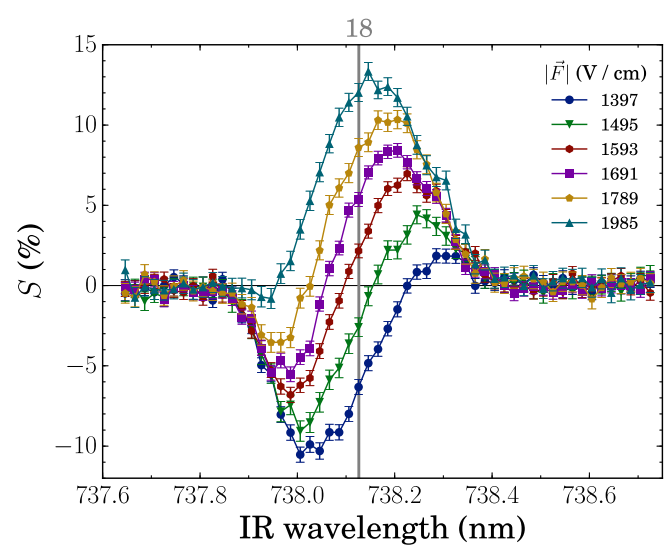

Fig. 20. Transitions to $n=18$ in a $63 \mathrm{~V} \mathrm{~cm}^{-1}$ electric field in the excitation region and fields outside the grid as indicated. The vertical line shows the position of the field free peak. Positive $S$ values indicate atoms in Stark states that cannot pass beyond the grid due to field ionization. From reference [125].

For each value of $n$, the ionization field for the outermost Stark state with a negative Stark shift in Ps is approximately equal to the classical ionization field [175] $F_{\text {ion }}=2 R_{\mathrm{Ps}} h c / e a_{\mathrm{Ps}} 9 n^{4}$, where $R_{\mathrm{Ps}}=0.5 R_{\infty}$ is the Rydberg constant for Ps, and $a_{\mathrm{Ps}}=2 a_{0}=1.058 \times$ $10^{-10} \mathrm{~m}$ is the Ps Bohr radius. This suggests an ionization field of $1360 \mathrm{~V} \mathrm{~cm}^{-1}$ for $n=18$, close to that observed for the transmission of the corresponding states in Figure 20. On the other hand, the outermost Stark states with positive Stark shifts ionize at approximately $2 F_{\text {ion }}$, indicating that a field of $2721 \mathrm{~V} \mathrm{~cm}^{-1}$ should be required to completely inhibit transmission through the grid for all states with $n=18$. In fact a lower field seems to accomplish this, which may be because the actual ionization rates have to be taken into account and included in the SSPALS time window analysis in order to properly explain the data. Another possibility is that the tunnel ionization rates in Ps are actually different from the equivalent (scaled) hydrogen rates because the physical mechanism is not fully analogous; work is ongoing to check this.

There are several experimental reasons why it might be desirable to produce Rydberg Ps, which are mostly connected to two of their properties, namely: (1) they live much longer than ground state atoms, because excitation effectively turns off the annihilation process and (2) they may be controlled with external inhomogeneous electric fields, because excitation can produce atoms with large dipole moments. Thus, exciting Ps atoms to Rydberg levels provides a way to turn a fast moving short-lived divergent gas into slow, long-lived, focused Ps beam. Rydberg Ps states can also be easily detected using MCP detectors, either by field ionization near the detector [138], or by direct collisions, owing to the $\approx 6.8 \mathrm{eV}$ internal energy. This will be useful for spectroscopy experiments in which atoms are promoted to long-lived states and transported to a spatially separated detection region where stray fields can be precisely controlled and atom trajectories mapped out [122].

Rydberg Ps atoms might exhibit interesting interactions that are qualitatively different from those of 
other Rydberg systems. This may occur because in most Rydberg systems an electron is promoted to a highly excited state, leaving the rest of the atom or molecule as a slow moving spectator, whereas in the case of Ps the electron and positron can both interact with external systems in similar ways. This may, for example, affect the way that Rydberg Ps atoms interact with surfaces [421-424]; such interactions can be exploited to study various processes, for example charge transfer [425-427] or measurements of electric fields $[428,429]$, and may also be of relevance to possible Ps interferometry experiments.

The production of antihydrogen by Ps collisions with antiprotons has been discussed for some time [430,431], and it has long been understood that the cross section for anti hydrogen formation via this interaction will increase if Ps atoms in excited states are used [418,419,432]. Rydberg Ps is clearly well-suited for such schemes since, in addition to the increased cross section, the long lifetimes will also allow for flexibility in an experiment [416]. Analogous processes can be studied without antiprotons via the charge conjugate reaction, i.e., hydrogen formation by Ps collisions with protons [433]. Similarly, one could use $\mathrm{He}^{+}$ ions (which are even easier to produce than protons [434]) to measure charge-exchange neutralization following Rydberg Ps collisions with the ions. A related scheme has recently been proposed to produce cold atoms following ion cooling in a neutral atom trap superimposed over an electrostatic ion trap [435].

\subsubsection{Fluorescence decay of Rydberg atoms}

As described in Section 2.1.1, the decay rate of Rydberg Ps atoms can be calculated in exactly the same way as for hydrogen [148]. That is, the fluorescence decay rate, $1 / \tau_{n \ell}$, of a pure- $\ell$ state may be found by summing the Einstein $A$ coefficients associated with all allowed decay pathways to lower-lying states. This rate is usually dominated by decay to the ground state, and thus we can expect higher-lying states to live longer. For states with low values of the angular momentum quantum number $(\ell)$, the fluorescence lifetimes will scale with $n^{3}$. However, in typical experimental conditions we expect to produce only $\ell$-mixed Stark states of Ps, for which the Ps lifetime will have a different $n$ dependence (cf. Fig. 2). Thus, even though the decay rates for any given state can be calculated with high accuracy (or at least, accurately enough for any presently feasible experiment), the exact nature of the mixed states produced in real experimental conditions is not always precisely known, in which case it is useful to measure the Ps lifetimes.

The configuration shown in Figure 21 was used to measure Ps lifetimes by detecting Rydberg Ps atoms $1.2 \mathrm{~m}$ away from the Ps source [134]. Ps atoms typically have speeds on the order of $10^{7} \mathrm{~cm} \mathrm{~s}^{-1}$ (see Sect. 2.2.1) and will therefore travel on average $\approx 0.2-3.5 \mathrm{~m}$ over typical (mean) lifetimes for Rydberg states within the experimentally accessible range $(n=10-30$, cf. Fig. 19). For this reason it is necessary to allow the Rydberg atoms to fly along a different pathway from that of the incident positron beam. This was accomplished by bending the positron beam with the guiding magnetic field (which is

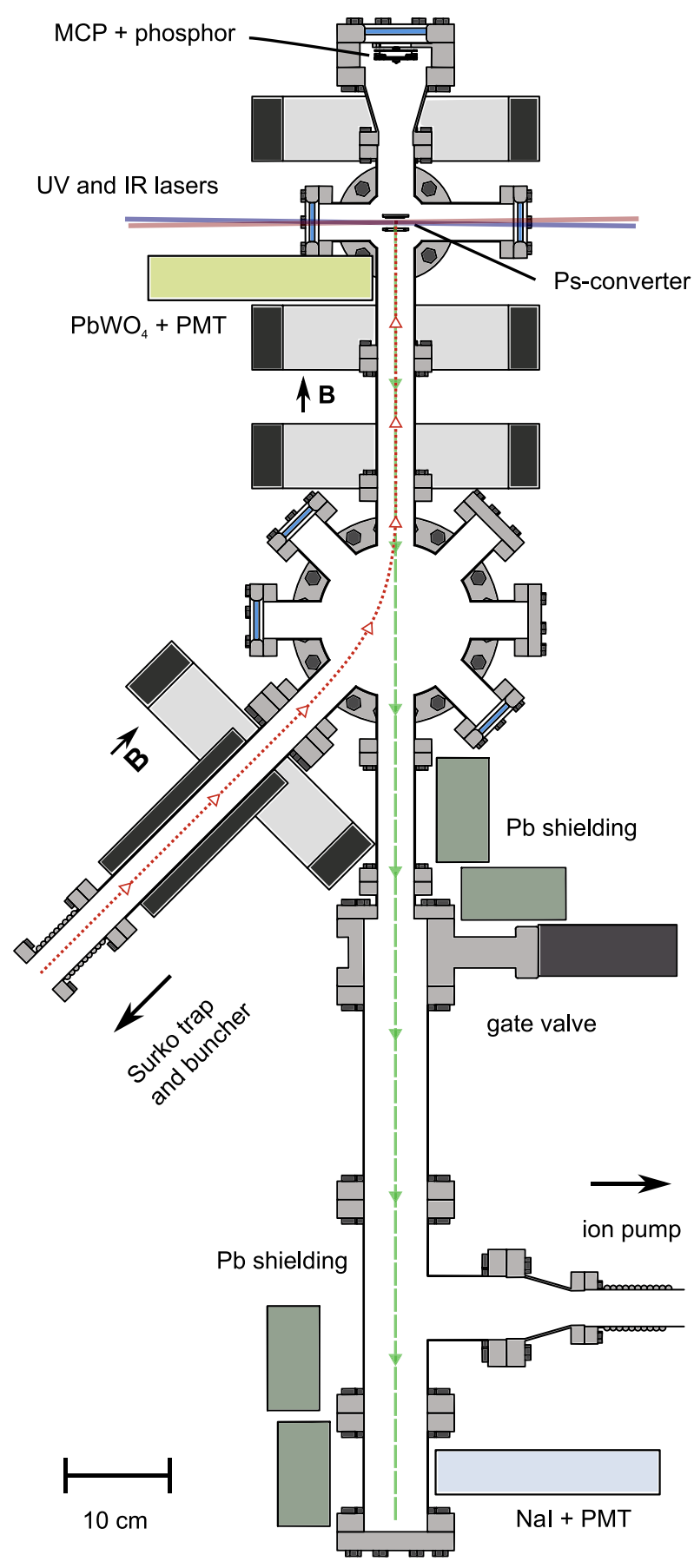

Fig. 21. Experimental arrangement used to measure Rydberg fluorescence lifetimes via Ps time-of-flight. Positrons from the Surko trap (red dots) are guided by a magnetic field through an angle of $45^{\circ}$ to a mesoporous silica target. Ps atoms emitted from the target are excited to long-lived states and some fraction of them travel $1.2 \mathrm{~m}$ along the straight flight path (green dashes) to annihilate near the NaI+PMT gamma-ray detector. From reference [134].

not expected to have any significant impact on the Ps atoms). It would also have been possible to implant the positrons at a $45^{\circ}$ angle [122], although this increases the spatial extent of the Ps source by $\approx 40 \%$. 
Ps atoms were emitted from a mesoporous silica film with a relatively broad angular distribution [126]. Because of this, the fraction of atoms that travel along the flight path and are finally detected $1.2 \mathrm{~m}$ away is low, and on average less than one event was detected per positron pulse. This means that single-shot methods were not appropriate, and single event detection was used instead, employing a relatively slow $\mathrm{NaI}$ scintillator based detector. The output of this detector was directly connected to an oscilloscope, and an algorithm used (off-line) to register annihilation events based on pulse height and width criteria that are optimized to reduce background events [134]. This methodology is similar to standard positron annihilation spectroscopy (e.g. [80]) and can thus be performed using standard detectors. However, for measurements in which multiple events are expected in short time windows (see Sect. 3.2.2) it is beneficial to use faster detectors, such as those based on LYSO scintillators [328]. The detected events were compiled as a function of arrival time to generate TOF profiles, as shown in Figure 22.

The radiative lifetimes of Rydberg atoms could in principle be measured by trapping Ps atoms and observing the particle loss as a function of trapping time, as has been done with hydrogen [436]. This approach is not currently possible with Ps as trapping has yet to be achieved. Instead, fluorescence decay rates may be determined from TOF spectra, as shown in Figure 22. The number of events detected in the TOF profile will be reduced due to losses in flight following radiative decay, and subsequent annihilation. The profiles can therefore be used to infer the Ps fluorescence lifetimes for a range of $n$ states.

TOF profiles were measured for a series of $n$ values over a $20 \mu$ s time window. For lower $n$ states a significant amount of loss due to decay and annihilation in flight is expected, and for longer-lived states the loss rate will be commensurately lower. We assume that $n=20$ atoms have a negligible loss rate, and that the TOF profile for these atoms can therefore be considered to be the initial distribution. By fitting all of the TOF spectra simultaneously we then determine the fluorescence lifetimes for all states, as indicated in Figure 23. The large error bars present in this figure for the higher $n$ states occur because there is very little decay present in the corresponding TOF data (Fig. 22). Thus, these data are also much more sensitive to the assumed value of the reference $n=20$ spectrum, as indicated by the gray regions showing the variation for different choices of the $n=20$ lifetime. Conversely, the lower $n$ measurements are much less sensitive to this choice. Also shown in Figure 23 are calculated values for $\ell$ mixed states, averaged over all of the $m$ substates [134]. These data exhibit an $n^{4}$ dependence (and definitely not an $n^{3}$ dependence), in accordance with the expectation that the experimental conditions will result in significant $\ell$ mixing.

It is well known that Rydberg atoms can be strongly affected by blackbody radiation (BBR) [437-445]. Roomtemperature BBR has virtually no effect on low $n$ atoms and molecules and can usually be ignored, but in the case of Rydberg atoms the radiation field, characterized by photons with low frequencies $\nu \leq k T$ can significantly overlap with atomic transitions, and may also give rise

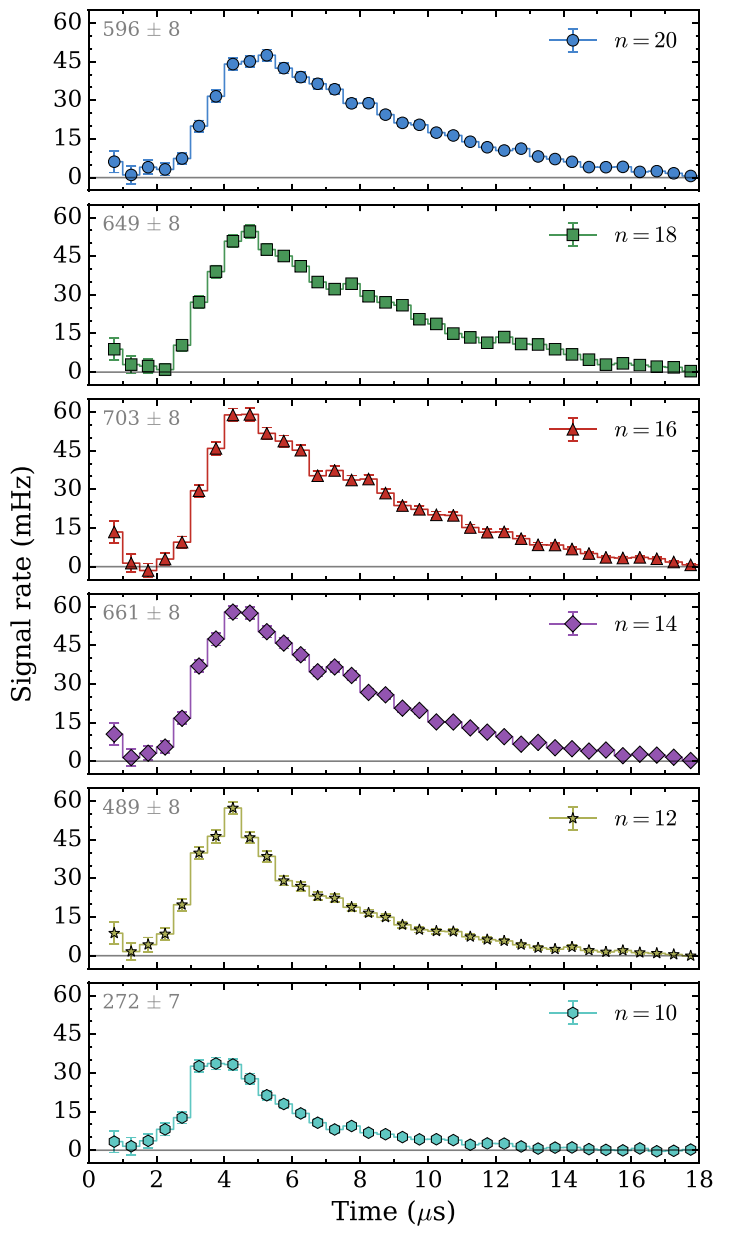

Fig. 22. TOF profiles used to determine flourescence lifetimes (only alternate $n$ values are shown). From reference [134].

to ac Stark shifts. Stimulated emission induced by BBR can lead to a redistribution of states among nearby levels, and thus may also cause a corresponding reduction in the radiative lifetime. For the Ps fluorescence lifetime measurements described above the greatest contribution to the rates of blackbody depopulation of the initially prepared states is expected to be via $\Delta n=1$ transitions. At room-temperature the time scales for $\Delta n=1$ blackbody transitions between $\ell$ mixed Rydberg states of Ps are approximately 20,50 , and $70 \mu$ s for states with $n=10$, 15, and 20, respectively [134]. Thus, the data shown in Figure 23 are expected to be largely unaffected by BBR effects. However, several future experiments are envisioned in which higher $n$ states and longer flight times will be employed, in which case BBR effects will have to be taken into account.

\subsubsection{Manipulation of Rydberg atoms with electric fields}

As discussed in Section 2.1.1, atoms and molecules in highly-excited Rydberg states may have large electric dipole moments (Eq. (39)), and therefore inhomogeneous electric fields may be used to apply forces to them (Eq. (40)). The potential energy of such atoms may increase or decrease when they enter an electric field, 


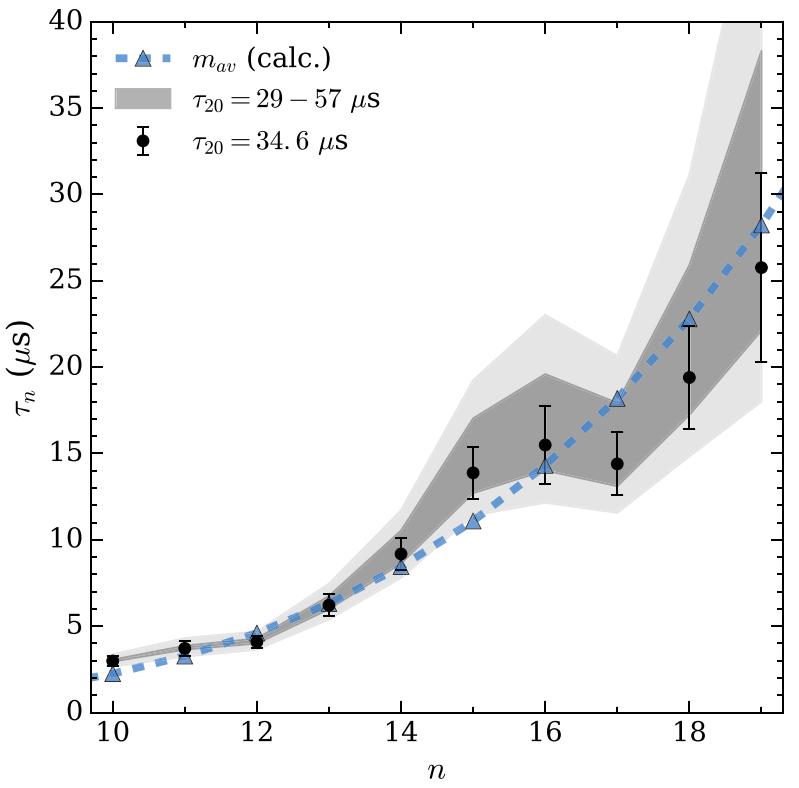

Fig. 23. Measurements of the mean lifetime for Rydberg Ps atoms with principal quantum number $n$ ranging from 10 to 19. The dark shaded region indicates the range of lifetimes obtained for different lifetime values assigned $n=20$ data as shown in the legend, with the corresponding error indicated by the light shaded region. Also shown are the calculated lifetimes averaged, over all $m$ states. From reference [134].

depending on their $k$-state (see Fig. 6). States with positive $k$ values gain energy in an electric field, and are therefore known as low field seekers (lfs) (i.e., they move to regions of lower field to minimize their potential energy). Conversely, states with negative $k$ values lower their potential energy in high fields, and are known as high field seekers (hfs). States with $k=0$ are to first order unaffected by external fields. For lfs, the gain in energy comes with a concomitant loss in kinetic energy, and vice versa for hfs. This process is the underlying mechanism by which the translational motion of Rydberg systems can be controlled via external fields.

The same principle applies to polar molecules; their permanent dipole moments can also be used to manipulate their motion with electric fields. This concept has been around for many years, for example, electric fields are used for state separation in the ammonia maser [446]. More recently, both static and time varying electric fields have been successfully employed in various schemes to produce cold molecules [447-451]. Molecular dipole moments are typically on the order of a few D, and $10 \mathrm{D}$ is considered to be extremely large. NB: the SI unit of the electric dipole moment is the Coulomb-meter ( $\mathrm{Cm})$; A more convenient unit, that will be used here, is the Debye (D), which is equivalent to $3.3 \times 10^{-30} \mathrm{Cm}$. Experimentally relevant Rydberg states can easily have dipole moments that are many orders of magnitude greater than those of polar molecules. Thus, applying the necessary fields to control the motion of atoms or molecules is consequently much easier to accomplish when working with Rydberg systems. Moreover, in principle any atom or molecule can be excited to a Rydberg state, and thus be produced with a large dipole moment.

Exploiting the very large dipole moments of Rydberg atoms and molecules in order to control their translational motion was first suggested by Wing [452] and Breeden and Metcalf [453]. The first experimental realization was accomplished by Softley and co-workers, who demonstrated the transverse deflection of beams of Rydberg $\mathrm{Kr}$ atoms [454] by a dipolar electric field. A few years later, longitudinal acceleration and deceleration of $\mathrm{H}_{2}$ beams using static electric fields was also achieved, by the same group [455]. The use of time-dependent electric fields to control Rydberg atoms and molecules was introduced by Vliegen et al. [456] in 2005. This work allowed control over the translational motion of hydrogenic [457] and non-hydrogenic [458] atoms, as well as the realization of a wide range of atom optics elements, including mirrors [459], lenses [457], deflectors [460,461], decelerators and traps [436,462-466]. These advances have been employed for a various studies, including the effects of blackbody induced transitions and photoionization of Rydberg states [463,467], $m$-changing dipole-dipole interactions in gases of polar Rydberg atoms and their effects on Rydberg state lifetimes [468], the preparation of long-lived high- $|m|$ (i.e., $|m| \geq 3$ ) Rydberg states of $\mathrm{H}_{2}$ [469,470], and new methods to study ion-molecule reactions at low temperatures [471,472].

Thus, the manipulation of atoms and molecules in Rydberg states via inhomogeneous electric fields is now a well-developed research field [338]. An array of techniques have been developed to facilitate experimentation with many different atomic and molecular systems, but it is only recently that these ideas have been applied to Ps [136]. This is because the production of Rydberg Ps atoms has only recently become routine (see Tab. 1). It is therefore very likely that in the near future we will see many of the techniques established to control atoms and molecules via the Stark effect applied to Ps. This includes deceleration and trapping using time varying fields, which are discussed further in Section 5.1.

The practical problems associated with applying established Stark deceleration and trapping methods to Ps atoms are mostly related to their scarcity, speed, and divergence. Direct application of standard chip-based electrode designs [460,465], for example, would likely result in devices with a low acceptance for Ps because of the spatial properties of typical Ps atom ensembles. This is not an unsurmountable problem however, and there is no obvious impediment to redesigning electrode structures to accommodate the properties of Ps atoms. Indeed, Ps atoms generated using standard methods typically have kinetic energies close to $50 \mathrm{meV}$ or less (see Sect. 2.2.1). This is in fact less than has been extracted from hydrogen [469] and helium [466] atoms, and strongly suggests that once acceptance limitations are compensated for, controlling Ps atoms may actually be easier than it is for other atoms, particularly since the low Ps mass implies that very large accelerations should be achievable.

The first experimental realization of the use of inhomogeneous electric fields to manipulate the motion of Rydberg Ps atoms took place in 2016 [136]. In this 

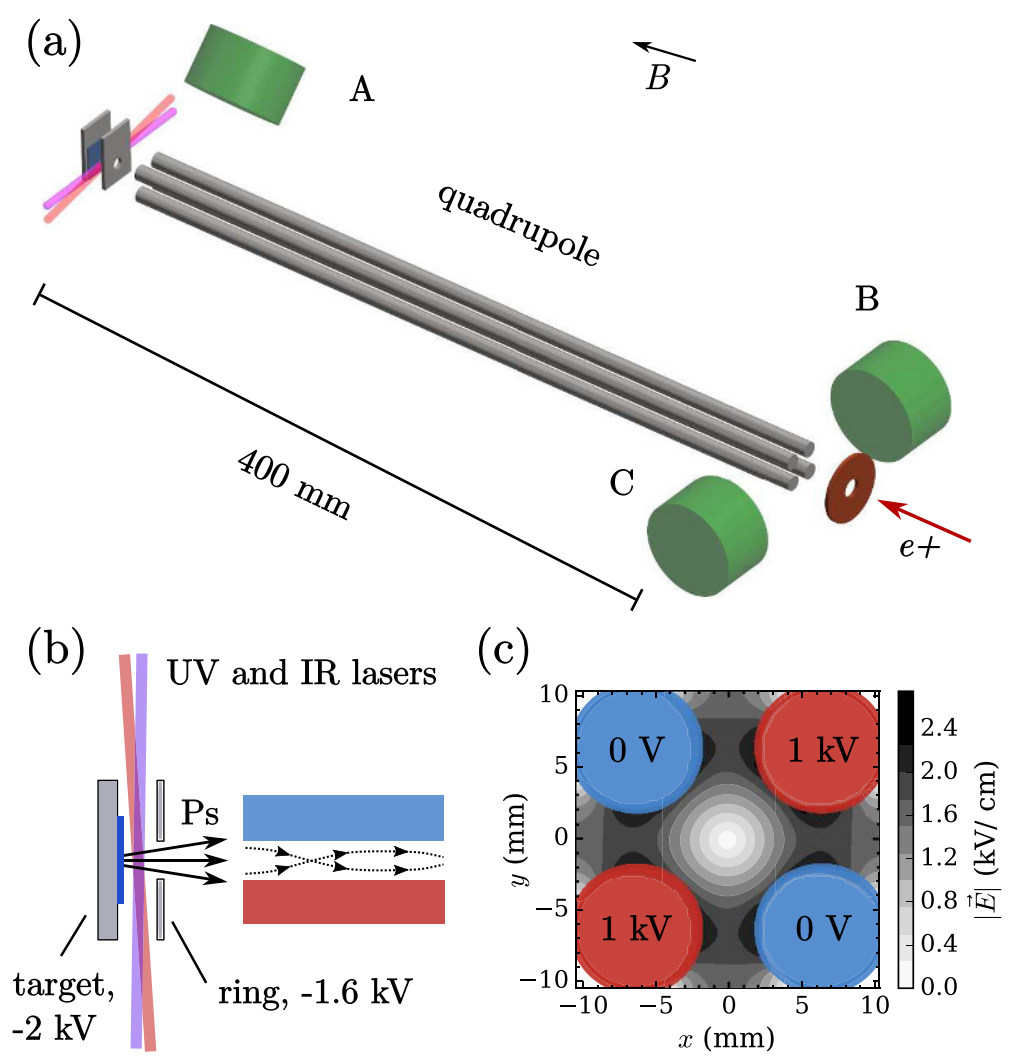

Fig. 24. (a) Schematic representation of the a quadrupole guiding experiment, indicating the Ps excitation region and the positions of the gamma-ray detectors A, B and C. (b) Schematic view of the excitation region and quadrupole entrance, and (c) a contour plot of the electric field inside the quadrupole electrodes. From reference [136].

experiment a positron beam was magnetically guided through a grounded quadrupole electrode. The positrons were then implanted into a silica film, and Ps atoms optically excited between electrodes that allow the electric field to be controlled, as described above. The guide fields were turned on before the Ps atoms could leave the excitation region, which can easily be achieved using solid-state high-voltage switches since the Ps atoms take $\approx 100$ ns to reach the grid (see Fig. 18). Ps atoms in Rydberg-Stark states with principal quantum number $n=10$ were prepared, which then entered the quadrupole guide structure: inside this device lfs states are deflected away from the electrodes by the inhomogeneous electric fields, and are thus guided longitudinally along the device. Conversely, hfs states are deflected away from the axis of the guide towards the electrodes and will be lost. A schematic of the experimental arrangement and the radial electric fields inside the quadrupole is shown in Figure 24. The electric field profile shown is for $1 \mathrm{kV}$ applied to one pair of rods, with the other two grounded.

The detector labeled A in Figure 24 was used to monitor the production of Rydberg states. In order to elucidate the efficacy of guiding for different $k$ states, an electric field of $670 \mathrm{~V} \mathrm{~cm}^{-1}$ was applied in the excitation region. This was necessary because the spectral resolution of the system was $\approx 130 \mathrm{GHz}$, mostly due to Doppler broadening and the effective laser bandwidth. Without broadening the lineshape all Stark states would be produced by the laser on resonance, and it would not be possible to investigate the guiding of different parts of the Stark manifold. The Stark broadened $n=2 \rightarrow 10$ spectrum is shown in Figure 25a along with calculated spectral intensities [336] for the individual $k$ states. The solid black line is a convolution of the calculated intensity distribution with a $130 \mathrm{GHz}$ Gaussian function, The laser polarization used in the experiment was such that excited states were produced predominantly with azimuthal quantum number $m_{\ell}=1$, and even values of $k$, The calculated relative spectral intensities [336] of the transitions to the $n D$ components of the $m_{\ell}=1$ Stark states are indicated by the vertical bars in Figure 25a. The applied field is not sufficient to resolve individual Stark states [125], but it does allow optical selection of predominantly lfs or hfs states.

Figure 25b shows the (background subtracted) total count rate measured by detectors $\mathrm{B}$ and $\mathrm{C}$ as a function of the IR laser wavelength and for different guide voltages. The data obtained with $0 \mathrm{~V}$ applied to the guide were recorded at the same time as the spectrum shown in Figure 25a. In this case the detection of atoms will be independent of the $k$ state, and indeed the observed count rate is consistent with the spectral line shape (that simply represents the total number of Rydberg atoms produced) and the solid angle for emission towards the guide within a narrow cone with a half angle of approximately $1^{\circ}$. When the guide fields were applied, however, the observed count rate exhibited a strong dependence on the $k$ states 


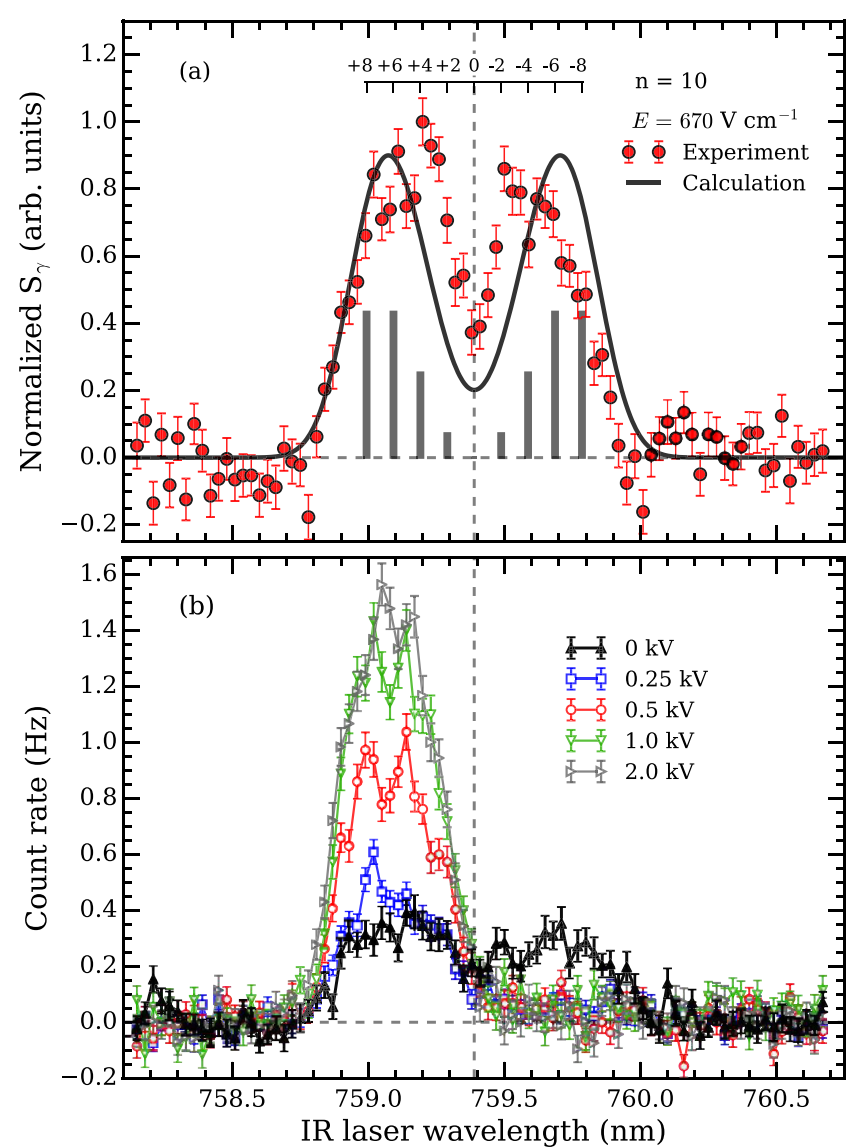

Fig. 25. (a) Lineshape $n=2 \rightarrow 10$ transitions as measured by single shot lifetime spectroscopy. The vertical bars indicate calculated relative spectral intensities of the $k$ states labeled by the horizontal scale. The black solid line is a convolution of these with the expected $130 \mathrm{GHz}$ spectral resolution. (b) Background subtracted count rate obtained from single event counting detectors for the indicated voltages applied to the quadrupole guide electrodes. The dashed vertical line indicates the field-free transition wavelength. From reference [136].

prepared. In particular, it was found that no hfs states were guided, demonstrating that even those states with the smallest electric dipole moments (i.e., $k=-2$ states with $\mu=150 \mathrm{D})$ were deflected out of the device, even at the lowest applied fields.

For lfs states enhanced Ps transport along the guide was observed, as indicated by the increased count rate in detectors B and C. At the lowest guide fields only the $k=+8$ states (with $\mu=610 \mathrm{D}$ ) were observed above the guide off background signal. In higher fields, atoms in states with smaller dipole moments were transported, as one would expect. The quadrupole structure shown in Figure 24 is the most basic implementation of Stark manipulation. This arrangement is quite effective because it need only provide radial confinement to function, and the acceptance of the device already selects for forward directed atoms. A slightly more useful version of this is a curved quadrupole device, as shown in Figure 26. This arrangement works in much the same way as the straight guide, but has the additional feature that the curvature of

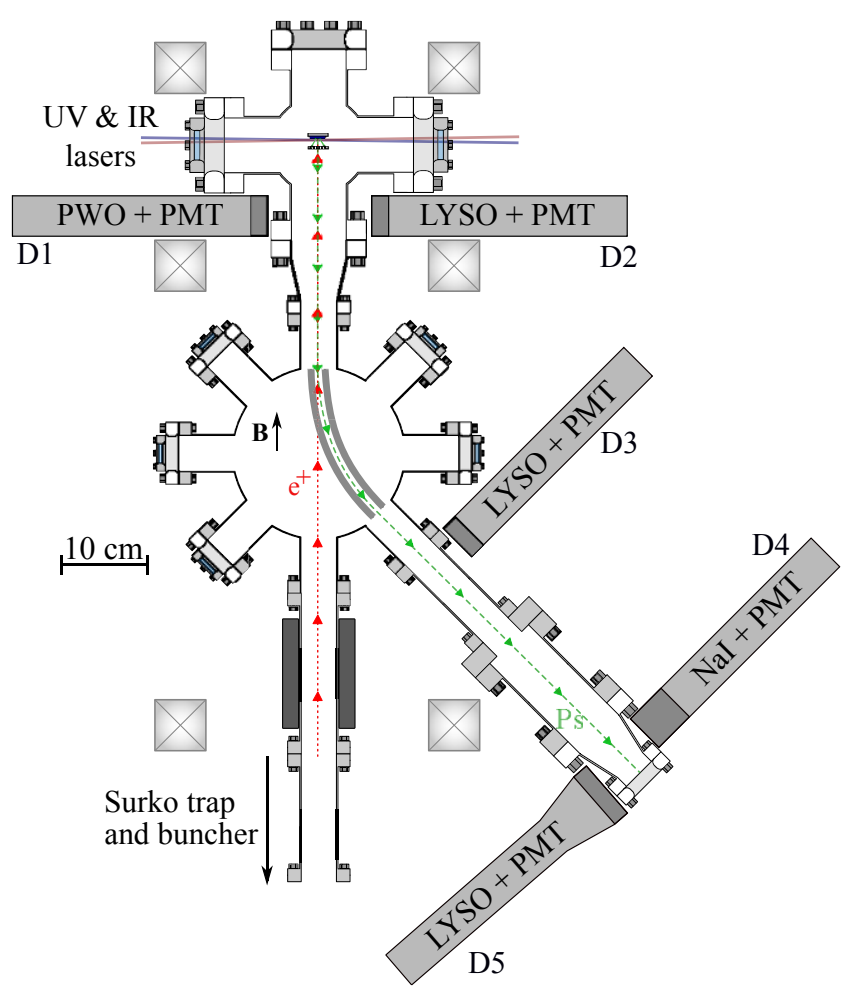

Fig. 26. (a) Schematic representation of the experimental apparatus containing the curved quadrupole guide. The positions of five gamma ray detectors used in the experiment are indicated. D1 and D2 are used to monitor Ps atoms in the excitation region via single-shot lifetime spectroscopy, whereas D3, D4, and D5 are used to generate single-event TOF spectra. The total length along the axis of the curved guide is $0.6 \mathrm{~m}$. From reference [140].

the device can transport Ps atoms off of the positron beam axis, and in the process can act as a velocity selector [140].

Figure 27 shows the normalized velocity profiles obtained using no guide (Fig. 21), a straight guide (Fig. 24), and a curved guide (Fig. 26). Note that all of these profiles, which are obtained from TOF data, have been normalized and do not represent the relative efficiency of Ps transfer. They do, however, show that the guide acts as a velocity selector, although it does not indicate the efficiency of any particular guiding arrangement. The data obtained with no guide (used to measure the fluorescence lifetimes) self-select faster atoms as only those atoms that happen to be traveling along a narrow emission cone will be detected. The quadrupole guides also select specific parts of the velocity distribution, based on the applied fields and the intrinsic acceptance of the devices.

The initial velocity distribution entering the curved guide can be approximated as falling somewhere between the no guide and straight guide cases [140]. The data in Figure 27 shows that the curved guide does indeed apply some velocity selection. This mechanism is in fact well-known from many experiments with polar molecules $[449,450,473,474]$, in which a cold beam can be extracted from a room temperature gas by velocity selection via a curved guide (often a hexapole rather than a quadrupole, 


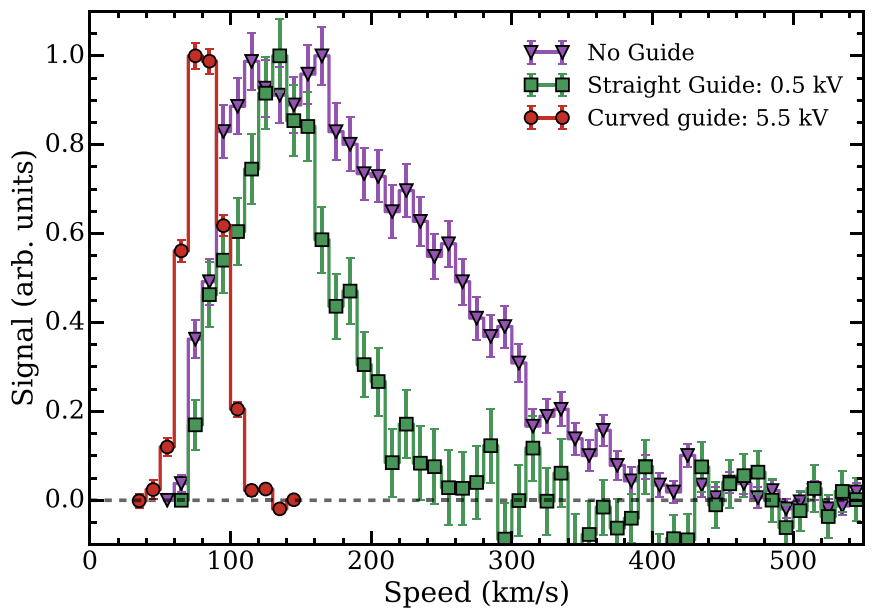

Fig. 27. Longitudinal velocity distributions as derived from TOF measurements obtained with no guide (triangles), a straight guide (squares) and with the curved guide (circles). The electric potential applied to the guide rods are indicated in the legend. From reference [140].

as they have some favorable properties [475]). This is possible only by extreme velocity selection, with losses of more than ten orders of magnitude. For ordinary gases this can still result in a useful particle flux, but in general velocity selection is not the preferred option for Ps experiments, since the number of atoms available is already severely limited. There may, however, be some specialist experiments for which it is advantageous to select a slower part of the distribution. For example, this may be the case in experiments designed to produce positron-atom bound states via Rydberg Ps collisions with atoms [476].

Figure 28 shows Rydberg Ps production (a) and transmission (b) in a curved guide, as a function of the IR excitation laser wavelength. As in the straight guide measurements, this makes it possible to observe the effect of selecting particular $k$ states (with some limited resolution). When no electric field is applied the laser bandwidth is such that all $k$ states are produced, in proportion to the relevant transition strengths, which are generally symmetrical with regard to positive and negative $k$ values $[125,336]$. Excitation in zero field is less efficient since it produces atoms in states that cannot be guided, which are therefore lost. These data show explicitly that it is beneficial to split the Stark manifold (by an amount determined by the available spectral resolution) and then tune the laser to the lfs side of the spectrum in order to optimize the production of lfs states. Note that the asymmetric lineshape observed with the electric field applied arises because of the deflection of atoms towards or away from D2 by the electric fields between the grid electrode and the quadrupole electrodes.

Another example of Ps atom control was recently implemented by researchers at the University of California, Riverside (UCR) [141]. In this work an electrostatic mirror was produced using 360 wires with alternating potentials applied, as shown in Figure 29. A sinusoidal potential on a planar or cylindrical surface leads to an electric field, $F$, the magnitude of which decays exponentially with
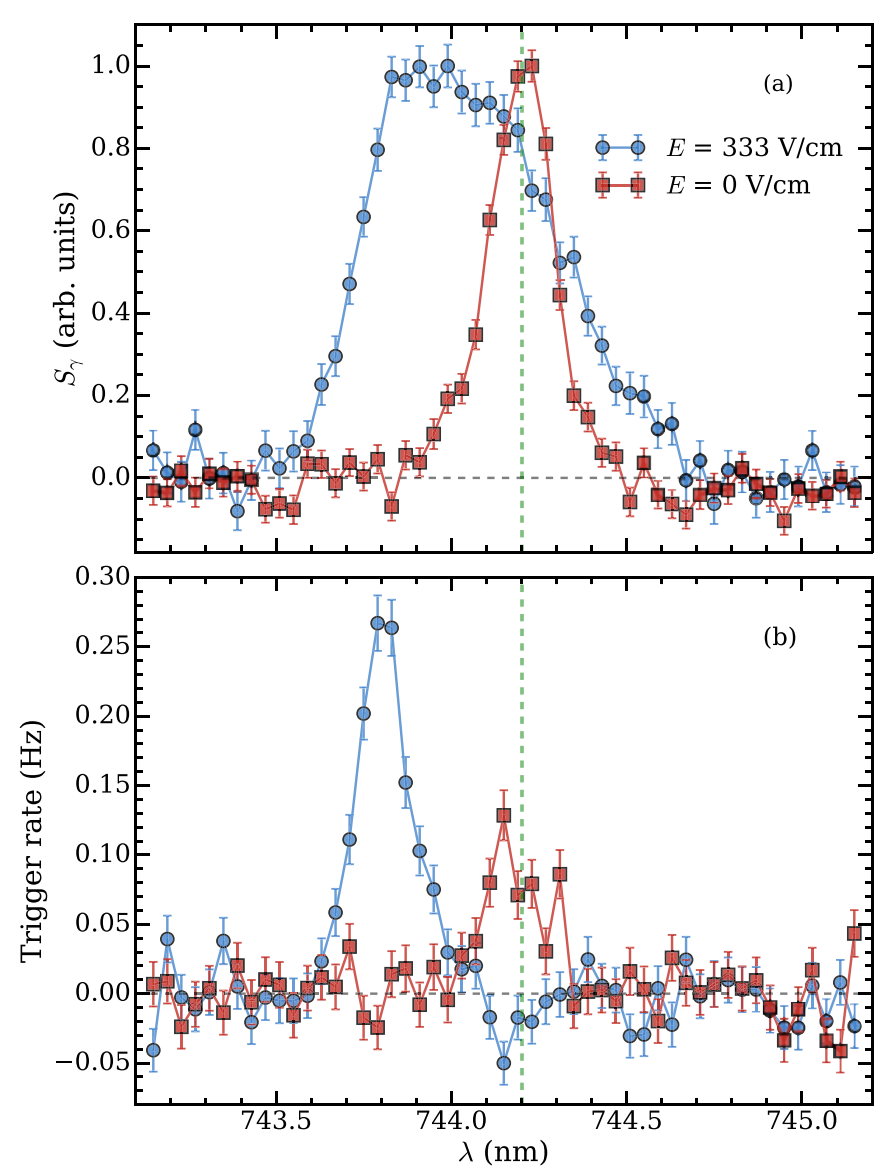

Fig. 28. (a) Spectrum of the $2{ }^{3} \mathrm{P}_{\mathrm{J}} \rightarrow 14^{3} \mathrm{~S} / 14^{3} \mathrm{D}$ transitions in the curved guide configuration shown in Figure 26. the data were recorded using detector D2 with and without a $333 \mathrm{~V} \mathrm{~cm}^{-1}$ electric field applied. (b) Total count rates for guided atoms as a function of the IR laser wavelength, measured by detectors D4 and D5. The dashed vertical line represents the expected zero-field resonant wavelength. From reference [140].

distance from the plane of the surface, thus leading to an effective potential for Rydberg atoms that is proportional to $|F|$ and so only depends on the distance above the plane. This property leads to specular reflection, and hence this arrangement forms a mirror surface.

Ps was produced by implanting positrons into a singlecrystal $\mathrm{Cu}(110)$ target heated to a temperature of $950 \mathrm{~K}$ [52]. Ps atoms were excited using the same two-color two-step excitation process described previously [132]. Ps emitted from the $\mathrm{Cu}(110)$ target was excited to Rydberg states with principal quantum number $n=32$, which will have a fluorescence lifetime of more than $200 \mu$ s [134]. Using a metal target rather than a mesoporous silica target has the advantage that the emitted Ps has a lower transverse energy spread (see Sect. 2.2.1). By aligning the excitation lasers perpendicular to the Ps emission direction the first-order Doppler spread is minimized, making it possible to resolve higher $n$ states.

The electrode structure shown in Figure 29 was placed near the center of a $6 \mathrm{~m}$ long vacuum chamber: Ps atoms were emitted from a $1.8 \mathrm{~mm}(\mathrm{FWHM})$ spot at one end of 


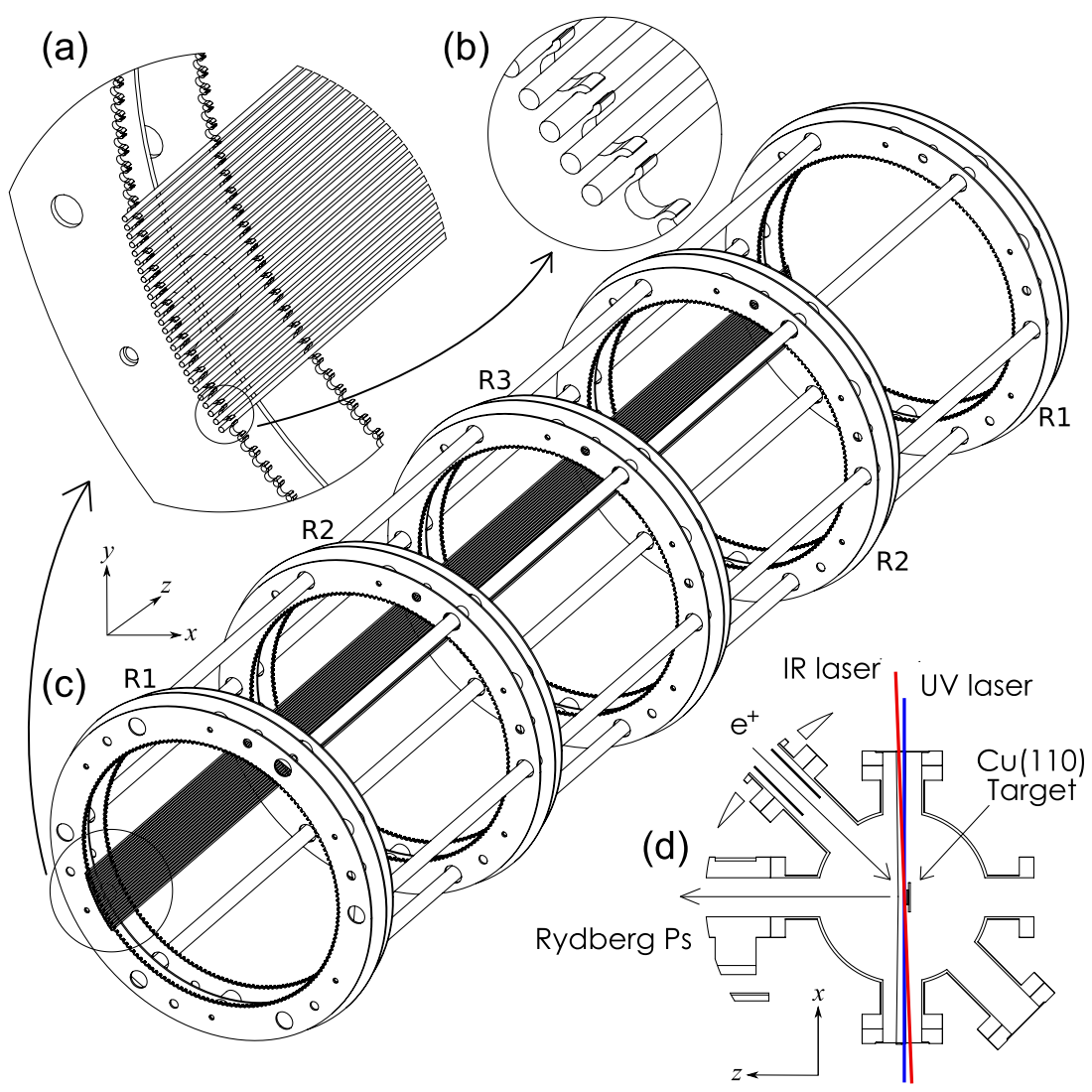

Fig. 29. Schematic representation of the UCR Rydberg atom-mirror construction and housing. (a) and (b) show how the 360 individual wires are attached, and in (c) the complete mounting structure is shown. The support rings hold the wires in elliptical arcs, with mean radii of $95.78 \mathrm{~mm}$ at $\mathrm{R} 1,96.56 \mathrm{~mm}$ at $\mathrm{R} 2$, and $96.98 \mathrm{~mm}$ at $\mathrm{R} 3$. The Ps formation and excitation chamber is shown in (d). Reprinted with permission from [141]. Copyright (2017) by the American Physical Society.

the flight tube, and were detected by a position-sensitive microchannel plate (MCP) Rydberg detector [138] $6 \mathrm{~m}$ away. This detector provides both spatial and temporal information, making it possible to observe beam focusing, and to simultaneously measure TOF spectra. The mirror comprises a wire structure $0.9 \mathrm{~m}$ long with a $96 \mathrm{~mm}$ mean inner radius, in the shape of a truncated oblate ellipsoid of revolution.

TOF spectra from the mirror experiment are shown in Figure 30, measured for applied potentials of \pm 95 and $\pm 10 \mathrm{~V}$, and with the electrodes grounded. These data show enhanced transport when the mirror is on. There is an extra enhancement of fast atoms for the higher fields, evidenced by the peak at $\approx 14 \mu \mathrm{s}$. This is caused by the arrival of direct Ps, which is much faster than thermal Ps (having kinetic energies of around $2 \mathrm{eV}$ ), with some contribution from UV induced secondary electrons and/or ions [138]. The $\approx 30 \mu$ s flight time over $6 \mathrm{~m}$ implies a Ps kinetic energy of $225 \mathrm{meV}$, approximately a factor of 3 times more energetic than one would expect from Ps thermally emitted from a $\mathrm{Cu}(110)$ target heated to $950 \mathrm{~K}$. This is due to velocity selection that favors faster atoms moving in the direction of the mirror. Note that these flight times are considerably longer than those observed in Figure 22, or any of the quadrupole guide experiments. This is because the UCR experiments employ higher $n$ states, a much longer flight path and slightly slower Ps atoms. For such long flight times and high $n$ states it is possible that BBR effects will be present (cf. Sect. 3.2.3).

A significant difference between the UCR mirror and the UCL quadrupole guide is the former acts more like a lens, insofar as it focuses the Ps beam to a small spot. This property opens up many additional experimental avenues, the most significant of which may be the possibility of performing a gravitational free-fall measurement [477] (see Sect. 5.5). In order to use such a device to measure gravity effects one would have to ensure that the mirror did not introduce any spurious deflections. Furthermore, since this device relies on atoms having large dipole moments, other stray fields (or, more properly, field gradients) would have to be reduced or eliminated. This is of course true for any gravity experiments that require manipulation of Rydberg atoms via external fields. The focusing effect of the UCR mirror is shown in Figure 31. These data show two-dimensional distributions as measured by the position sensitive MCP detector, averaged over different flight times between 20 and $100 \mu$ s with the mirror potentials on and off. Gaussian fits to the Ps image data indicate that the beam spot has a width of $32.2 \pm 0.9 \mathrm{~mm}$ (FWHM). Also shown is an optical focus (Fig. 31c), which has a Gaussian spot size of $21 \pm 3 \mathrm{~mm}$ (FWHM). The difference is attributed to the fact that the mirror is not precisely in 


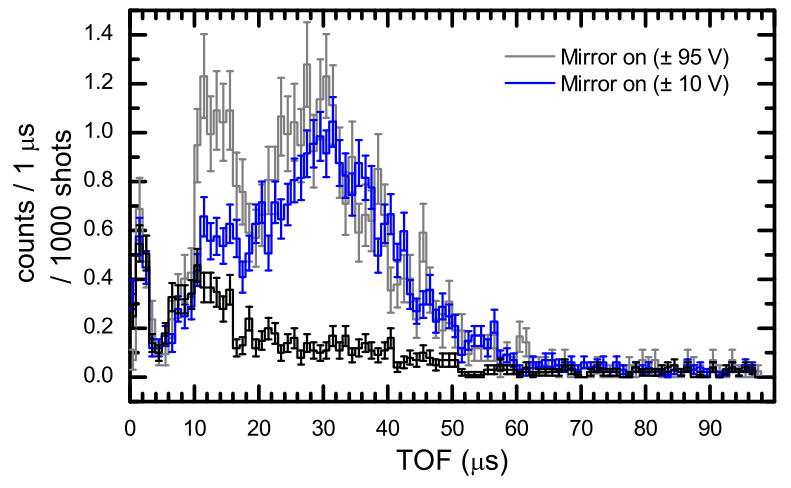

Fig. 30. TOF spectra for $n=32$ atoms measured with the Rydberg focusing mirror off, and with applied potentials of $\pm 10 \mathrm{~V}$ and $\pm 95 \mathrm{~V}$. The early peak at $\approx 14 \mu \mathrm{s}$ for the high voltage case is due to faster (direct) Ps emitted from the $\mathrm{Cu}(100)$ Ps source. The slower atoms arriving at $\approx 30 \mu$ s are caused by the slower thermally desorbed Ps atoms. Reprinted with permission from [141]. Copyright (2017) by the American Physical Society.

the center of the flight path. The size of the beam is not strongly dependent on the flight time, indicating that the focus is largely achromatic.

The alternating wire electrode structure used in the mirror might also be applied in some different configurations. For example, flat mirrors that confine slow Ps atoms in a box-like structure. The authors state that a mirror for back reflection should be able to back-reflect Rydberg Ps with energies less than $100 \mathrm{~K}$ [141]. Thus, one could envision constructing a Rydberg atom storage cell.

\subsubsection{High resolution Rydberg time-of-flight spectroscopy}

Measurements of the kinetic energy of positronium atoms via TOF methods (see Sect. 3.1.2) have been used even before slow positron beams were available [237]. The energy resolution of standard TOF methods is intrinsically limited by the Ps lifetime. There are other limitations that occur in the way these methods are usually implemented: for example, spontaneous decay events are usually observed through narrow apertures (e.g. [238]), with a concomitantly low detection efficiency. These limitations can be mitigated, e.g., by using multiple detectors [478], or by using lasers to detect Ps positions [126] (see Fig. 14). However, the $142 \mathrm{~ns}$ lifetime of ground state atoms will typically limit the energy resolution to the \% level. For example, if one allows Ps atoms to fly for three lifetimes (426 ns) and annihilation events can be measured to within a few ns, the effective energy resolution will be on the order of a few $\%$.

Ps momenta can also be measured using ACAR: these systems, however, are not very common and have an energy (or momentum) resolution determined by the angular resolution of the position sensitive gamma ray detectors [479], which is increased by locating them very far from the annihilation radiation source, resulting in a low count rate and long data acquisition times (typically days). The angular resolution of ACAR systems is limited

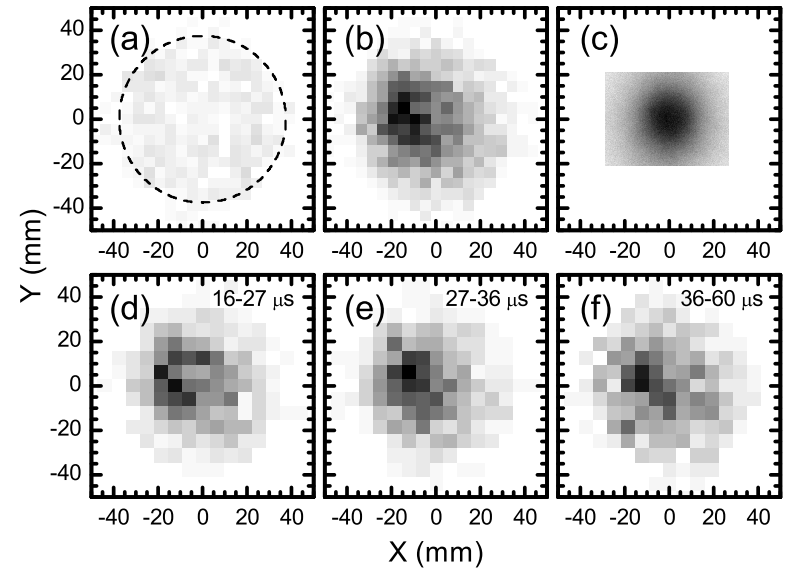

Fig. 31. 2D histograms of the position data of $n=32$ Rydberg Ps incident upon the detector with (a) the mirror off and (b) the mirror on with $\pm 10 \mathrm{~V}$ applied. In (c) the focus achieved using light is shown for comparison. In $(d, f)$ the data of $(b)$ are subdivided into 3 velocity groups with the indicated flight times, illustrating that the mirror focus is largely free of chromatic aberrations. The dashed circle in (a) indicates the extent of the active area of the MCP. The color scales represent the total number of counts detected per unit area, where white means zero counts, and black indicates the maximum signal. Reprinted with permission from [141]. Copyright (2017) by the American Physical Society.

by practical reasons to approximately $0.1 \mathrm{mR}$, which also yields an energy resolution of the order of a few $\%$.

The energy resolution from Ps TOF measurements can be dramatically improved if the atoms are excited to Rydberg levels. These atoms can live for orders of magnitude longer (see Sect. 3.2.1), and thus the energy resolution can be correspondingly higher, if it is not limited by other factors. Performing high resolution TOF measurements has been achieved by the UCR group [124]. In this work Ps atoms were excited to states with $n=30$, and were detected by a micro channel plate detector [138] at the end of a $1.78 \pm 0.01 \mathrm{~m}$ flight path. Flight times ranging from 3 to $10 \mu$ s could then be determined to within $\approx 10$ ns. The increased flight times give an improvement in energy resolution of a factor of 10 or so.

This improved energy resolution has enabled the observation of Ps emission from Bloch states in metal organic framework (MOF) crystals [480], including the identification of the emission energies related to specific features of Ps-crystal interactions [124]. MOF materials are compounds similar to zeolites, consisting of metal or clusters bound to organic molecules to form highly porous and very stable structures, which may be crystalline [481]. They are of general interest for use as hydrogen storage media, catalysts, drug delivery vehicles, and so on. Their structures are of interest to positronium physics because Ps atoms inside them exist in a delocalized state [301] known as a Bloch wave $[482,483]$. This can effectively distribute the Ps mass over a large area in the crystal structure, which can affect the Ps energy distribution. The high energy resolution in the UCR experiment made it possible to observe a narrow energy distribution of $250 \pm 10 \mathrm{meV}$ for 


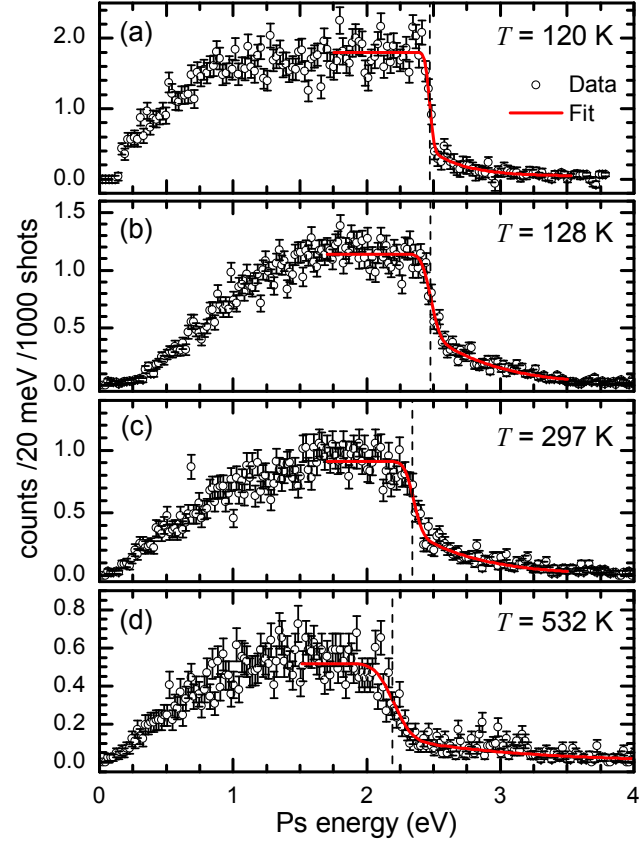

Fig. 32. Spontaneous Ps emission energy spectra for a positron implantation energy of $5 \mathrm{keV}$ (a) and $3 \mathrm{keV}(\mathrm{b}-\mathrm{d})$, and $\mathrm{Cu}(111)$ target temperatures as indicated in the panels. The solid lines are fits to the data and the dashed vertical lines indicate the $50 \%$ cut off point of the edge as determined from the fits. Reprinted with permission from [137]. Copyright (2016) by the American Physical Society.

Ps emitted from a material known as ZIF-8 cooled to $80 \mathrm{~K}$ [124]. This may be of use in Ps-laser experiments since it is the large energy spread, and associated Doppler broadening, that necessitates the use of broad-band lasers. Since there are many different types of MOF crystal it may be possible to find Ps sources with narrower energy spreads. It is worth pointing out that previous measurements using conventional Ps TOF methods were not able to resolve such energy features [302].

Processes that remove a single electron from metal surfaces can in some cases act as probes of the electronic structure of that surface. The most well-known example of this is angle-resolved photoemission (ARPES) [484], but a similar role may be played by Ps $[234,485]$ if the momentum of the emitted Ps atom is measured, either by TOF or ACAR methods. As discussed in Section 2.2.1, in some metals it is energetically possible for a thermalized positron to form a bound state with an electron just inside the surface, and be emitted as a Ps atom. This direct Ps formation can occur if the sum of the electron and positron workfunctions is less than the Ps binding energy. That is, the emitted Ps energy will be $-\phi_{\mathrm{Ps}}$ (Eq. (19)), i.e., $\phi_{\mathrm{Ps}}=\phi_{+}+\phi_{-}-E_{B}$,

This process is expected to predominantly leave a single hole behind in the metal, just as it does for ordinary electron photoemission [234]. It may even be that there are fewer perturbing effects in the Ps emission process, since it involves an electrically neutral particle. In any case, Ps emission can be viewed as being directly analogous to angle resolved photoemission spectroscopy. However, previous experiments in this area $[234,255,485]$ have not been competitive with standard techniques such as ARPES in terms of the available energy resolution. An experiment using a high-resolution TOF arrangement was performed to measure the kinetic energy of Ps directly emitted from a $\mathrm{Cu}(110)$ surface [137]. Figure 32 shows energy spectra measured for different temperatures. The solid lines are fits using a thermally broadened step with half point located at the maximum Ps emission energy given by a density functional theory calculation that includes positron states in the solid [486]. These data give the Ps energy $-\phi_{\mathrm{Ps}}=2.476 \pm 0.001_{\text {stat }} \pm 0.0013_{\text {sys }}$ measured at $128 \mathrm{~K}$, in very good agreement with the theory.

\subsection{Doppler-free two-photon transitions}

The hydrogen atom has long been the testing ground for quantum physics owing to its simplicity; as a one-electron atom $\mathrm{H}$ is extremely well described by the Dirac equation, and its properties have been calculated with sufficient accuracy that even the structure of the proton has to be considered [487]. NB: these types of corrections have received greater scrutiny in recent years because of the muonic hydrogen results [488] discussed in Section 5.2. As it is so well understood theoretically the hydrogen atom is an excellent basis for metrology [489], especially in combination with frequency combs [490]: the hydrogen 1S-2S interval has been measured to 4.2 parts in $10^{15}$ [491]. Ps is also a simple atomic system that can be well described theoretically, which means that it too is an interesting system upon which to perform precision measurements. Doing so, however, requires overcoming some extreme challenges. Many of them are a direct result of having to deal with low numbers of very fast atoms that are able to self-annihilate.

All precision spectroscopic measurements require eliminating or significantly reducing Doppler effects [492], but the situation is obviously more extreme in the case of Ps, where even second order Doppler effects, which are proportional to $(v / c)^{2}$, may have to be taken into account. The underlying physical reason for second order Doppler shifts is relativistic time dilation, and as such there is no way to eliminate them other than by using slower atoms. For $1^{3} \mathrm{~S}_{1} \rightarrow 2^{3} \mathrm{P}_{J}$ transitions, second order Doppler effects would result in line-broadening comparable to the $50 \mathrm{MHz}$ natural linewidth [108]. The $1^{3} \mathrm{~S}_{1} \rightarrow$ $2{ }^{3} \mathrm{~S}_{1}$ experiments of $\mathrm{Chu}$, Mills and co-workers used two counter-propagating beams of $486 \mathrm{~nm}$ light to drive two-photon transitions, which eliminates the first order Doppler broadening [493-495]. The unavoidable second order Doppler shifts were taken in to account by performing measurements with atoms at different speeds, and extrapolating to zero. Ordinarily only the most precise measurements require accounting for second order Doppler shifts [491], and the need to do so with Ps represents a serious limitation on possible precision Ps spectroscopy. If in future measurements extrapolating to zero velocity is no longer sufficient, more accurate measurements of the velocity profiles may be obtained to allow for more sophisticated Doppler corrections [122]. Clearly, 
developing colder Ps sources is also highly desirable for such experiments.

Nevertheless, even if one had access to an intense source of ultra-cold Ps atoms, it would still not be possible to approach the extreme precision that has been achieved in hydrogen measurements because the natural linewidth of the $1^{3} \mathrm{~S}_{1} \rightarrow 2^{3} \mathrm{~S}_{1}$ transition in Ps is determined by its annihilation lifetimes. Since these scale with $n^{3}$, and the ground state lifetime is $\sim 142 \mathrm{~ns}$, the natural width is given by

$$
\Gamma_{1 \mathrm{~s}-2 \mathrm{~s}}=9 /(16 \pi \times 142 \mathrm{~ns})=1.26 \mathrm{MHz} .
$$

The equivalent width in atomic Hydrogen is a million times smaller $(\sim 1.3 \mathrm{~Hz})$, determined by the $0.12 \mathrm{~s}$ lifetime of the metastable $2 \mathrm{~S}$ state [188]. The radiative lifetime of the $2^{3} \mathrm{~S}_{1}$ state of Ps is twice as long as this, but this is quite irrelevant as the annihilation lifetime is $1.136 \mu \mathrm{s}$.

The experiments by $\mathrm{Chu}$ et al. [103] were performed using a pulsed beam derived from a magnetic bottle bunching system [102]. This arrangement was able to capture around $100 \mu \mathrm{s}$ of the output of a CW beam with $4 \times 10^{5}$ positrons per second. After time-bunching [345] a $10 \mathrm{~ns}$ with pulse of roughly 20 positrons was implanted into an $\mathrm{Al}(111)$ Ps production target [52]. The resulting Ps atoms were then irradiated with pulsed laser light, leading to the absorption of two $486 \mathrm{~nm}$ photons, and excitation of the metastable $2 \mathrm{~S}$ state and subsequent photoionisation, owing to the high laser intensity used. The extent to which the excitation laser will also ionize depends on the power, and the relative excitation and ionization cross sections at the wavelength in question. As the laser intensity increases, excitation and ionization rates compete, eventually reaching a steady state which for $486 \mathrm{~nm}$ light results in a theoretical maximum in the production of excited states of $17.6 \%$ [496].

However, in some experiments [103] ionization is desirable, since the correlation of the laser wavelength with the detection of ionized positrons can be used as the experimental signal. An example of such data are shown in Figure 33. Despite the low number of positrons available this experiment was able to measure the $1^{3} \mathrm{~S}_{1} \rightarrow 2^{3} \mathrm{~S}_{1}$ interval to $1233607185 \pm 15 \mathrm{MHz}$, which is an uncertainty of only 12 parts per billion (ppb). The line center frequency was calibrated using a molecular Tellurium line, which was subsequently found to be slightly mis-calibrated [497]. When this and other appropriate corrections were applied [498] the measured interval was found to be $1233607218.9 \pm 10.7 \mathrm{MHz}$ (an uncertainty of $8.7 \mathrm{ppb}$ ).

A second generation of $1{ }^{3} \mathrm{~S}_{1} \rightarrow 2{ }^{3} \mathrm{~S}_{1}$ experiments were completed, almost ten years after the first measurements $[107,108]$ which included significant improvements, including (1) a high-intensity accelerator based positron beam [346], (2) colder positronium atoms, (3) an improved detection scheme [106], and (4) a CW laser and a highfinesse $\left(10^{5}\right)$ Fabry-Pérot build up cavity [108].

The magnetic mirror trap initially used by Mills [102] had a storage time of around $100 \mu \mathrm{s}$. Although it may be possible to extend the storage time of such a device [499], the most direct way to improve the number of positrons per pulse is to use a more intense source. The development

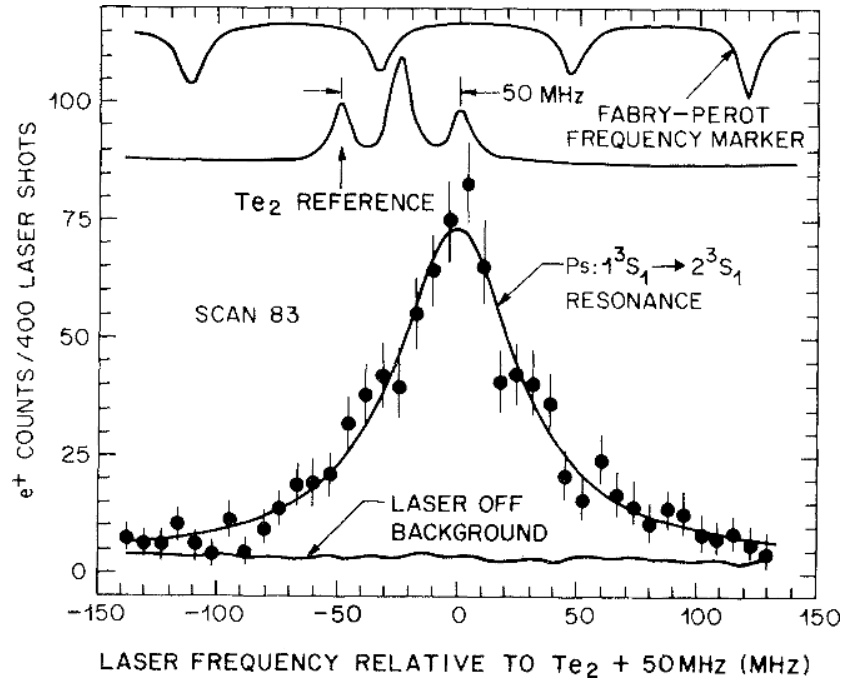

Fig. 33. Resonant three-photon REMPI of positronium, comprising a two-photon Doppler free excitation, and subsequent photoionization from the same $486 \mathrm{~nm}$ light. The line center was $25.9 \pm 2.7 \mathrm{MHz}$ above the $\mathrm{Te}_{2}$ reference line. The lopsided nature of the figure is as it was originally published. Reprinted with permission from [103]. Copyright (1984) by the American Physical Society.

of a free electron laser using a microtron accelerator at Bell labs [500] made it possible for Mills and co-workers to construct a parasitic positron beam, and thereby to generate pulses approximately $16 \mu$ s long containing $7 \times 10^{4}$ positrons [346]. Although such parasitic operation comes at a price [501], this approach was nevertheless able to deliver $10 \mathrm{~ns}$ wide positron pulses to the target at $30 \mathrm{~Hz}$, each containing $2 \times 10^{4}$ positrons.

The possibility of using colder Ps made the idea of using a CW laser to perform the experiments much more attractive [108]. Although preliminary tests suggested that the thermal Ps emitted from oxygen covered $\mathrm{Al}$ cooled to $235 \mathrm{~K}$ [235] would be compatible with a CW experiment, it turned out that the surfaces were quite sensitive to laser light and could not be used for long-term experimentation; ultimately a hot $(576 \mathrm{~K}) \mathrm{Al}$ target was used in the measurements [107].

The detection methodology was modified to reduce background counts from positrons that are re-emitted directly from the sample. This was particularly important in the $\mathrm{CW}$ experiment owing to the lower excitation efficiency. The background was reduced by separating the excitation and photoionization regions [106]. This meant that re-emitted positrons (the major source of background events) could not cross the magnetic field lines and were restricted to one side of the target, while long-lived $2 \mathrm{~S}$ atoms were able to drift across the field lines below the target level, where they could be photoionized. These liberated positrons were then able to travel to a MCP detector behind the target. Scattered positrons from the incoming beam were suppressed with skimmers and grids, all of which resulted in an improved signal to noise ratio of factor of 100 . 
Pulsed lasers are not the ideal choice for precision spectroscopy because the high instantaneous power leads to non-linear effects that can be difficult to properly model, leading to uncertainties in the expected lineshape (e.g. $[344,502])$. In order to implement a CW excitation laser Fee and co-workers used a Fabry-Pérot cavity to circulate several $\mathrm{kW}$ of laser power, providing $\sim 1.7 \mathrm{MW} / \mathrm{cm}^{2}$ of $486 \mathrm{~nm}$ light at the beam focus [108]. In this way an improved measurement of $1233607216.4 \pm 3.2 \mathrm{MHz}$ was obtained, giving an uncertainty of $2.6 \mathrm{ppb}$ [107].

Note that even with major improvements made to the experimental set-up, the final uncertainty only decreased by a factor of 3.3. This highlights the inherent difficulties associated with precision experiments involving Ps. The primary sources of uncertainty in the measurements were (1) the Tellurium reference line $( \pm 0.6 \mathrm{MHz})$, and (2) modeling the lineshape $( \pm 1.5 \mathrm{MHz})$. The former problem can now be easily dealt with since optical reference sources have improved significantly and with modern frequency comb technology uncertainties in the laser frequencies will not limit the precision of any realistic Ps measurement $[490,503]$. The latter is largely due to the Ps itself (extrinsic properties) and not any fundamental difficulties involved in modeling. Statistical limitations $(0.9 \mathrm{MHz})$ are inevitable in Ps experiments, and taking the second order Doppler shifts into account correctly required accurate knowledge of the Ps motion and the properties of the excitation and ionization lasers. Once again the need for a source of cold Ps atoms is evident.

The ultimate limitation in this measurement will arise from the $1.3 \mathrm{MHz}$ natural width. If the lineshape were very well known, it might be possible to determine the line center (or to "split the line") to a part in $\sim 10^{3}$ of the natural width. This would allow the $1^{3} \mathrm{~S}_{1}-2^{3} \mathrm{~S}_{1}$ interval to be measured with a precision of the order of $1 \mathrm{kHz}$. Such splitting is possible: the Ps hyperfine measurements have uncertainties of a few $\mathrm{MHz}$, while the natural width is on the $\mathrm{GHz}$ scale [156].

There are currently some new experiments in progress that aim to improve the $1^{3} \mathrm{~S}_{1}-2^{3} \mathrm{~S}_{1}$ uncertainty $[76,123,504]$. At the time of the previous experiments the theoretical uncertainly $(2.6 \mathrm{MHz}$ [505]) was comparable to the experimental value $(3.2 \mathrm{MHz}, 2.6 \mathrm{ppb}$ [107]). More recent calculations have reduced the theoretical uncertainty to $1 \mathrm{MHz}(0.8 \mathrm{ppb})$ and it would therefore now be beneficial to reduce the experimental uncertainty at least to the $100 \mathrm{kHz}$ regime (i.e., $0.1 \mathrm{ppb}$ ). Advances in positron trapping technology [69], Ps production [111,124,282] and laser metrology [490] offer some hope that higher precision will be achieved.

\section{Optical excitation of positronium ions and molecules}

In 1946, John A. Wheeler suggested that a series of entities composed only of positrons and electrons might exist, entities that he called "polyelectrons" [16]. This name may be somewhat misleading since both Ps and $\mathrm{Ps}^{+}$are polyelectrons, and yet contain only one electron. This apparent misnomer occurs simply because the
Table 5. Binding or dissociation energies $\left(E_{\mathrm{B}}\right)$, annihilation lifetimes $\left(\tau_{a}\right)$, and principal decay modes $(\mathrm{n} \gamma)$ for all of the polyelectrons thought to possess bound states, as well as the year they were discovered. $\mathrm{Ps}_{2}^{*}$ refers to the $L=1$ excited state predicted by Varga and coworkers [510]. The properties given are calculated values, as explained and referenced in the text.

\begin{tabular}{lllll}
\hline & $E_{\mathrm{B}}(\mathrm{eV})$ & $\tau_{a}(\mathrm{~ns})$ & $\mathrm{n} \gamma$ & Year observed \\
\hline $\mathrm{p}-\mathrm{Ps}$ & 6.803 & 0.125 & 2 & $1951[17]$ \\
$\mathrm{o}-\mathrm{Ps}$ & 6.802 & 142 & 3 & $1951[17]$ \\
$\mathrm{Ps}^{-}$ & 0.327 & 0.479 & 2 & $1981[509]$ \\
$\mathrm{Ps}^{+}$ & 0.327 & 0.479 & 2 & Not observed \\
$\mathrm{Ps}_{2}$ & 0.435 & 0.225 & 2 & $2007[360]$ \\
$\mathrm{Ps}_{2}^{*}$ & $<0.435$ & 0.442 & 2 & $2012[119]$ \\
\hline
\end{tabular}

positron was often referred to as a positive electron for some years after its discovery. One might imagine using the term "polyleptons" rather than polyelectrons, but this is also unrepresentative of what is meant as this group should then include, for example, muonium, the bound state between an electron and an antimuon, which are also leptons. The nomenclature is not of any great importance, and in deference to Wheeler we continue to use polyelectrons.

The first member of the polyelectron series is positronium itself, made from an electron and a positron. This is followed by the positronium ion, made from either two electrons and one positron $\left(\mathrm{Ps}^{-}\right)$or two positrons and one electron $\left(\mathrm{Ps}^{+}\right)$. The next member is the four particle system $\mathrm{Ps}_{2}$, comprising two electrons and two positrons. At the time Wheeler was not able to show that the $\mathrm{Ps}_{2}$ system was stable against breaking up into two Ps atoms, so the discussion stopped there, although the implication was that, at least in principle [506], one could simply keep adding electrons and positrons and construct ever more complex polyelectrons. Shortly after Wheeler's paper was published, Hylleraas and Ore used a variational method to show that $\mathrm{Ps}_{2}$ was in fact stable [507]. In this calculation the binding energy against breaking up into two Ps atoms was found to be $0.11 \mathrm{eV}$, somewhat lower than the current value of $0.434 \mathrm{eV}$ [508], as one might expect from a variational calculation with a limited basis.

Today there are many more theoretical methods available to determine the properties of polyelectrons, including not only their stability but also their binding energies and decay rates. Stability in this sense does not imply that the annihilation process will not quickly destroy the system. In terms of the (classical) orbital motion of the electrons and positrons, these systems may undergo many oscillations before they annihilate, and so may be regarded as having stable bound states, and a concomitant atomic structure. In other words, a distinction is drawn between the stability against break-up of a bound state, and stability against annihilation (a condition that is not fulfilled by any polyelectron). We now know that $\mathrm{Ps}_{\mathrm{s}}, \mathrm{Ps}^{ \pm}$and $\mathrm{Ps}_{2}$ are all stable. Not only have there been numerous modern calculations that show this but, with the exception of $\mathrm{Ps}^{+}$, all of these polyelectrons have been observed experimentally, as indicated in Table 5 . 
Variational calculations [511] suggest that $\mathrm{P}_{3}$ is not stable against dissociation. Similar calculations also imply that the $\mathrm{Ps}_{2} \mathrm{e}^{-}$ion is also not stable [512] (in disagreement with Ref. [513]). Thus, it seems likely that Wheeler's stable polyelectron series does not extend beyond the twoelectron, two-positron $\mathrm{Ps}_{2}$ molecule, leaving the $\mathrm{Ps}^{+}$ion as the only unobserved member. As discussed in Section 1, Ps was first observed by Deutsch in 1951 [17], using positrons emitted from a radioactive source in a gas-filled vacuum chamber. The observation of $\mathrm{Ps}^{-}$had to wait until the development of mono-energetic positron beams, and these ions were not observed until the experiments of Mills in 1981 [509]. Similarly, the production of $\mathrm{Ps}_{2}$ also had to await new technological developments, namely the Surko trap [66,69]. Intense positron pulses [356] derived from a Surko trap made it possible to generate interacting Ps atoms [287], leading to the observation of $\mathrm{Ps}_{2}$ formation in 2007 [360]. It is likely that $\mathrm{Ps}^{+}$ions were created in subsequent $\mathrm{P}_{\mathrm{S}_{2}}$ photoionization experiments [119], but no unambiguous $\mathrm{Ps}^{+}$signal could be obtained in that work.

\section{1 $\mathrm{Ps}^{-}$spectroscopy}

As a relatively simple three-body system, $\mathrm{Ps}^{ \pm}$ions represent an interesting challenge to theorists. In terms of their fundamental properties (e.g., lifetime, or the existence, or lack thereof, of bound excited states) there is no difference between positive and negative $\mathrm{Ps}_{\mathrm{s}}$ ions since particle and antiparticle pairs are found experimentally to be exactly the same. In general, therefore, there is no need to discuss $\mathrm{Ps}^{+}$and $\mathrm{Ps}^{-}$ions separately. Experimentally, however, limited access to positrons means that $\mathrm{Ps}^{-}$ions are much easier to produce, and will be the focus of the following discussion.

Structurally, $\mathrm{Ps}^{-}$ions can be thought of as a Ps atom with an attached electron [514]. Since the three bodies all have the same mass, the Born-Oppenheimer approximation [515] is not applicable, requiring the development of more advanced methods (e.g. [516,517]). Ps ions have been the subject of much theoretical work since Wheeler's paper [16], including studies of their decay rates [514,518-520] and binding energies [521-526].

The most accurate calculated $\mathrm{Ps}^{-}$decay rate is $\Gamma=$ 2.087963(12) ns ${ }^{-1}$ [520]. The spatial $\mathrm{Ps}^{-}$wave function is symmetric with respect to the two electrons, and so for the total wave function to be antisymmetric the electrons must be in a singlet state. Therefore, when the positron interacts with an electron the probability of forming a singlet state is $25 \%$, just as it is when a positron is implanted into a solid material. Then we would expect the Ps ion decay rate to be approximately the same as the spin averaged Ps decay rate, or $\approx 2 \mathrm{~ns}^{-1}$. Obviously, the same argument applies to $\mathrm{Ps}^{+}$. One could also imagine a system where all three leptons had the same spin orientation, leading to a long lived triplet-like state. However such an arrangement is not bound because the electrons with the same spin must avoid each other due to the Pauli exclusion principle, leading to an increase in the electron kinetic energy, and a decrease in the net Coulomb attraction. The mean inter-particle distances in $\mathrm{Ps}^{-}$are 5.49 and 8.54 $a_{0}$ for the electron-positron and electron-electron pairs, respectively [527], supporting the idea that a $\mathrm{Ps}^{-}$ion is similar to a Ps atom with a loosely bound electron.

Very precise calculations of $\mathrm{Ps}^{-}$energies have been performed, yielding $E=-0.2620050702329801077703745$ a.u. [525]. This amounts to $\approx 7.1295 \mathrm{eV}$, and means that the dissociation energy required to produce a triplet (singlet) Ps atom and a free electron is $0.327(0.326) \mathrm{eV}$. NB: in Table 5 the triplet number is given as this is three times more likely to be produced [528].

Ps ions do not have any excited states, but they do possess a rich structure of resonances which have been the subject of many theoretical investigations, using a variety of techniques (e.g. [529-537]). Excitation of these resonances can result in electron detachment, which means that one may, in principle, study them via photo-induced changes in Ps ion decay rates in much the same way as in atomic Ps spectroscopy. However, as Ps ions are difficult to produce experimentally, such measurements represent a formidable challenge.

$\mathrm{Ps}^{-}$ions, were first produced in the laboratory by Mills in 1981 [509], who also measured their decay rate [538,539], obtaining $\Gamma=2.09(9) \mathrm{ns}^{-1}$. These experiments used the beam foil production method [242], which involves passing a positron beam through a thin $(\approx 2 \mathrm{~nm})$ carbon foil. This method is not very efficient, converting only $0.028 \%$ of the incident positrons into $\mathrm{Ps}^{-}$ions [509]. The same methodology as developed by Mills was used in further decay rate measurements carried out by researchers at Heidelberg University [540-542], who obtained $\Gamma=2.0875(50) \mathrm{ns}^{-1}$, in very good agreement with the calculated value of $\Gamma=2.087963(12) \mathrm{ns}^{-1}$ [520].

In all of these experiments a d.c. positron beam was used to produce $\mathrm{Ps}^{-}$ions that are immediately accelerated by a large electric field. The presence of ions is then detected by a Doppler shift in the subsequent annihilation radiation that depends on the applied acceleration field. The $\mathrm{Ps}^{-}$ion lifetime was measured by placing a second thin foil at a fixed distance after the initial foil. Energetic ions passing through the second foil are stripped, and may produce a free positron, detection of which indicates the number of ions that survive traversing the gap. For a given $\mathrm{Ps}^{-}$ion energy, the flight time can be modified by changing the gap between the two foils, allowing the decay rate to be measured. Because of the low number of positrons that actually form Ps ions, time-tagging methods such as those used in beam based PALS measurements [80] would not work well for lifetime measurements.

In the last decade an improved method to produce $\mathrm{Ps}^{-}$ ions was developed by Nagashima and co-workers in Tokyo [543-545]. This method is better suited to spectroscopic investigations since it is much more efficient, and the Ps ion producing surfaces are less delicate: it is unlikely that a few nm thick carbon foil would be able to survive repeated irradiation from intense laser pulses. The new approach uses alkali metal layers on tungsten surfaces to generate Ps ions with an efficiencies of more than $1 \%$. This work has recently been reviewed in detail [77] so we need not consider the production mechanisms here. In short, lowering the surface dipole barrier with monolayers of alkali metal coverage [546-548] makes it easier for a free surface positron to form a bound state with two electrons, and 

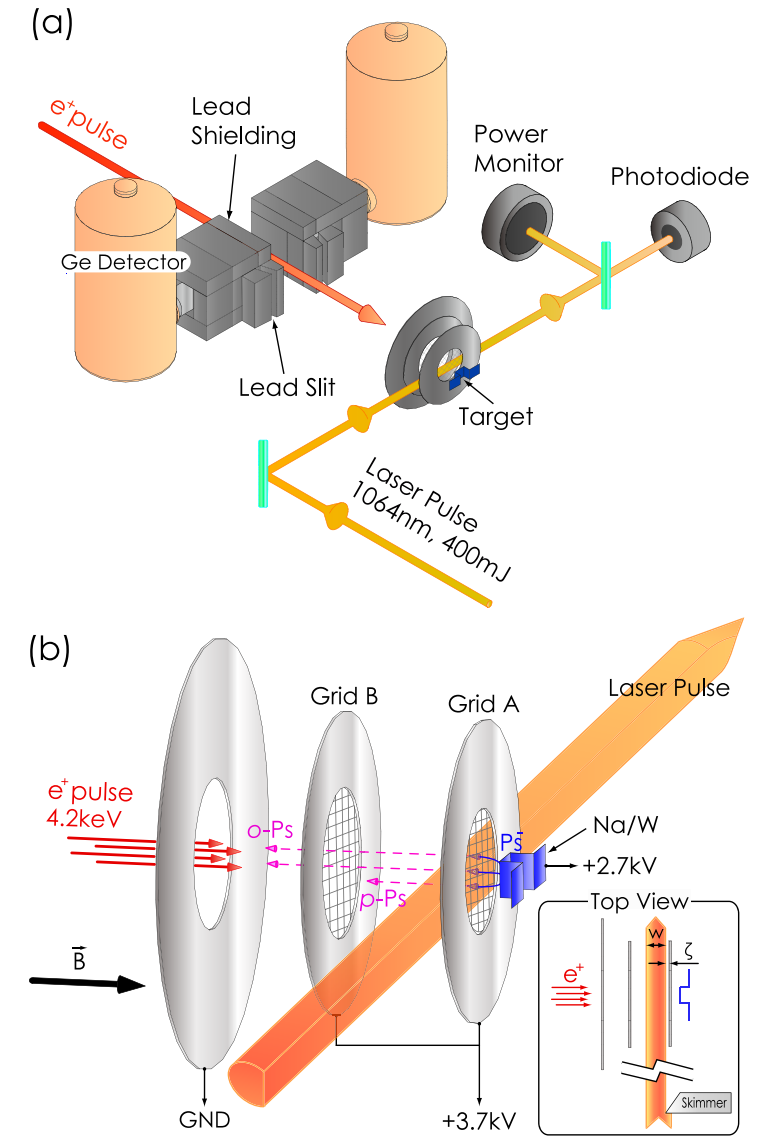

Fig. 34. Schematic of the $\mathrm{Ps}^{-}$photodetachment experiment showing (a) the positron beam, lasers and radiation detector layout and (b) the electrodes used to accelerate the ions before irradiation. The Ps ions are accelerated to $1 \mathrm{kV}$ before entering the field-free region where the photodetachment occurs, Doppler shifting $511 \mathrm{keV}$ photons to $529 \mathrm{keV}$. Lead shielding ensures that the radiation detectors observe only annihilation photons from the decay of Ps atoms resulting from Ps ion photodetachment, with a count rate on the order of 1 per 10 pulses. Reprinted with permission from [116]. Copyright (2011) by the American Physical Society.

thus increases the $\mathrm{Ps}^{-}$production efficiency: that is, the $\mathrm{PS}^{-}$"work function" (see Sect. 2.2.1) is reduced. Efficiencies up to $1.5 \%$ have been observed from $\mathrm{Na}$ and $\mathrm{K}$ coated W(100) surfaces, which can be used for many days [545]. This breakthrough has enabled many new $\mathrm{Ps}^{-}$ experiments to be performed [77].

The $0.326 \mathrm{eV}$ Ps ion dissociation energy means that the energy threshold for photodetachment is in the far infra-red $(\lambda=3.8 \mu \mathrm{m})$. The photodetachment cross section is of course zero at threshold [549] (in contrast to photoionization cross sections which are maximal) and has a maximum at nearly twice the threshold energy $(0.64 \mathrm{eV}$, or $\lambda=1.8 \mu \mathrm{m})$. The total photodetachment cross section has been calculated by Igarashi [550], who found that the maximum value is $\approx 6.5 \times 10^{-17} \mathrm{~cm}^{2}$, falling off to $\approx 4.4 \times 10^{-17} \mathrm{~cm}^{2}$ for $1064 \mathrm{~nm}$ light, which is the fundamental output of a Nd:YAG laser and thus easily produced in the laboratory. This calculation agrees with that of

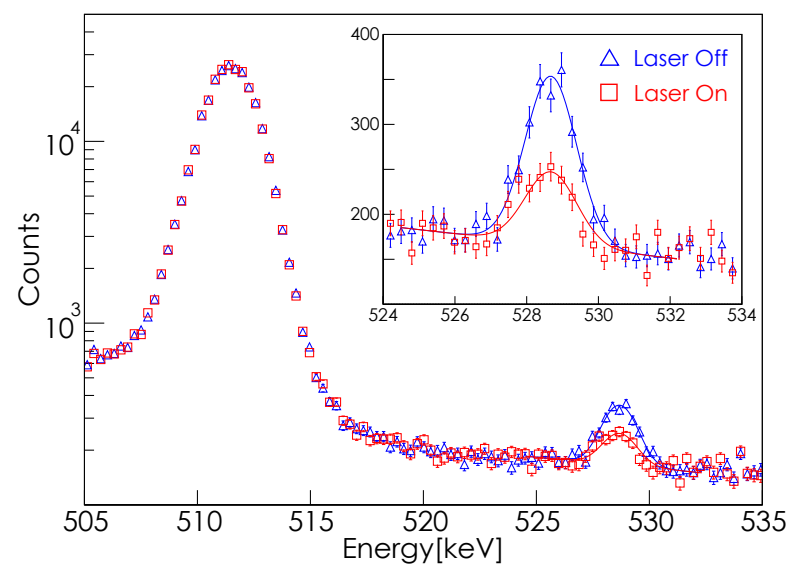

Fig. 35. Annihilation gamma radiation energy spectra with and without $1064 \mathrm{~nm}$ laser light present in a $\mathrm{Ps}^{-}$photodetachment experiment. The inset shows the Doppler shifted Ps ion peak on a linear scale. The solid lines are fits and the spectra are normalized to number of contributing incident positrons. Reprinted with permission from [116]. Copyright (2011) by the American Physical Society.

Ward et al., [551] but is almost 25\% lower, than that of Bhatia and Drachman [552]. In any case, this cross section is comparable to the photoionization cross section for $n=2$ Ps with $532 \mathrm{~nm}$ light [106] which has been experimentally demonstrated and is achievable using standard laser systems.

$\mathrm{Ps}^{-}$photodetachment has been demonstrated, also by the group of Nagashima in Tokyo [116]. This experiment was conducted at the KEK linac facility [349,553], which provides a $50 \mathrm{~Hz}$ pulsed positron beam with approximately $10^{4}$ positrons per $12 \mathrm{~ns}$ pulse. The experimental arrangement used in this experiment is shown in Figure 34 [116]. The signature of $\mathrm{Ps}^{-}$production in this experiment was again the Doppler-shifted annihilation radiation peak; reduction of this peak following laser irradiation indicates Ps photodetachment. The peak will not be affected if the detachment results in a singlet Ps atom as the speed will be largely unaffected, and the decay into two photons will therefore continue to exhibit the same Doppler shift. However, because the $\mathrm{Ps}^{-}$system contains two electrons of opposite spin the final state probability has the same distribution as the formation of Ps atoms with unpolarized electrons and positrons: that is, the possible spin configurations suggest that the triplet to singlet ratio will be $3: 1$, as implied by the fact that the Ps ion decay rate is (almost) the Ps spin-averaged lifetime [528]. Thus $75 \%$ of the Ps atoms produced will be in the triplet state, which decay into three photons, and therefore do not significantly contribute to the Doppler shifted $511 \mathrm{keV}$ peak. Figure 35 shows the diminished ion peak observed in the experiment when the photodetachment laser was present [116].

Analysis of the data shown in Figure 35 can be used to obtain a lower limit on the photodetachment cross section [116]. A limit rather than an absolute value was obtained because of the presence of various mechanisms (e.g., $\mathrm{Ps}^{-}$self-annihilation before it reaches the laser, the 


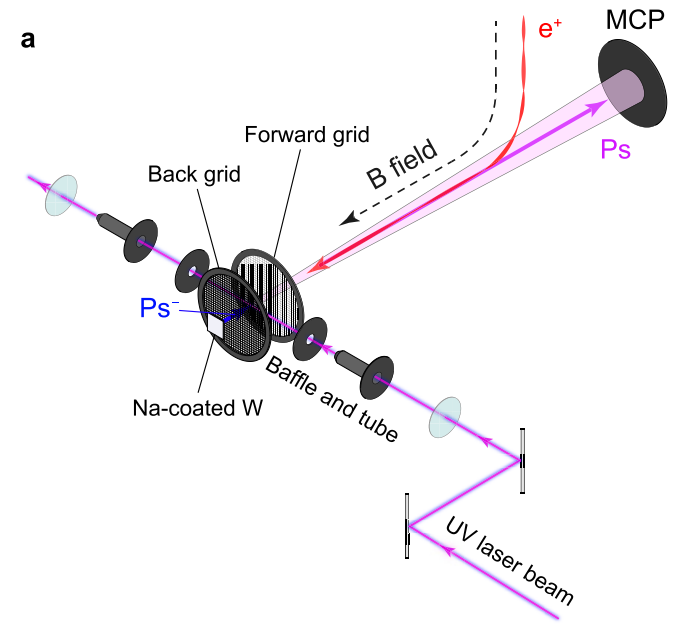

b

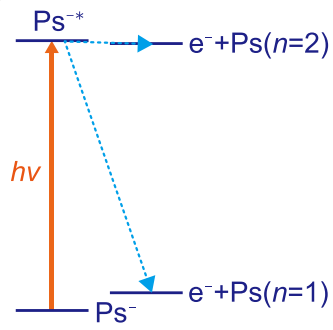

Fig. 36. (a) Schematic layout of the apparatus used to observe shape resonances in Ps ${ }^{-}$ions. Slow positron pulses are magnetically guided towards a $\mathrm{Na}$ coated $\mathrm{W}$ target, from which $\mathrm{Ps}^{-}$ions are emitted. The ions are accelerated by an electric field and then enter a field free region where they are irradiated by an ultraviolet laser light pulse. Neutral Ps atoms produced by resonant photodetachment are subsequently detected by a MCP detector $0.88 \mathrm{~m}$ away. (b) The partial level diagram indicating the optical transition from $\mathrm{Ps}^{-}\left({ }^{1} \mathrm{~S}^{e}\right)$ to both ground and excited $\mathrm{Ps}+\mathrm{e}^{-}$continuum states via the ${ }^{1} \mathrm{P}^{o}$ shape resonance. From reference [133].

exact ion-laser overlap, the laser shot-to-shot power distribution, and so on). The value obtained for the total photodetachment cross section was $\sigma \geq 2.1 \times 10^{-17} \mathrm{~cm}^{2}$, which is consistent with all calculations [550-552].

In some respects $\mathrm{Ps}^{-}$ions are similar to hydrogen negative ions. The equal mass of the positrons and electrons, however, has significant consequences for the $\mathrm{Ps}^{-}$atomic (or perhaps molecular [530]) structure. For example, while $\mathrm{H}^{-}$has a doubly excited ${ }^{3} \mathrm{P}^{e}$ state [549], no such state exists in the $\mathrm{Ps}^{-}$case [554], and indeed Ps ions are not thought to possess any stable excited states. However, there are expected to be a series of resonance states associated with the Ps Rydberg levels [529-537] whose presence affects the shape of photodetachment cross sections near threshold via interference effects [555]. This means that one can study these resonances by precise measurements of Ps excitation (and subsequent dissociation) at wavelengths close to the Ps excited states. Such an experiment has been performed by the Tokyo group.

The experimental methodology, indicated in Figure 36, was similar to that of the original photodetachment experiments. In this case, however, UV radiation was used to excite $\mathrm{a}^{1} \mathrm{P}^{o}$ resonance that exists close to the Ps $n=2$ threshold: as the authors of reference [133] explain, a strong shape resonance is thought to exist just above, and a series of Feshbach resonances just below, the $n=$ 2 threshold [529]. The experiment employed a different detection scheme from the previous measurements: rather than observing annihilation radiation (which intrinsically limits the count rate to less than 1 per pulse), the Ps atoms produced following $\mathrm{Ps}^{-}$-laser interactions were detected directly on an MCP detector (see Fig. 36). This was possible because the Ps ions were accelerated to kinetic energies of several $\mathrm{keV}$, so that the resulting Ps atoms striking the detector had more than enough energy to generate secondary electrons.
Figure 37 shows the results of $\mathrm{Ps}^{-} \mathrm{UV}$ irradiation experiments for two different Ps ion acceleration voltages. Here the parameter $R_{\mathrm{Ps}}$ is a background subtracted TOF count rate measured by the MCP detectors. The observed resonances have been fitted with a Fano profile [555] that has been successfully used to describe similar resonance profiles in $\mathrm{H}^{-}$experiments [556]. The fit also takes into account broadening and a shift of the lineshape due to the Doppler effect, which is significant in this measurement because of the high Ps ion energy. Overall the data yield a resonance energy of $5.4737(1) \mathrm{eV}(\lambda=228.07 \mathrm{~nm})$ for the zero velocity transition, which is consistent with theory $[529,550,557]$.

The cluster of data points in Figure 37 at energies just below the resonance suggests that an (unsuccessful) attempt was made to observe the below-threshold Feschbach resonances. Mills has suggested that these narrow resonances were not seen in the experiment because of Doppler broadening, even though the analogous resonance can be easily observed in $\mathrm{H}^{-}$measurements (cf. Fig. 5 in Ref. [556]). Improvements to the experimental procedure may resolve this issue, for example, by studying resonances using a Doppler-free two-photon scheme [558].

An obvious consequence of Ps ion acceleration and photodetachment is the production of an energy tunable Ps beam. This was first suggested by Mills [509], but has only recently been demonstrated following the experimental advances made by the Tokyo group (see Fig. 36) [120,559]. Energy tunable Ps beams can be made by passing a positron beam through a gas cell [85,231,560,561], or by glancing angle positron scattering [562]. These methods can generate beams in the energy ranges of approximately $5-500 \mathrm{eV}$ and $10-100 \mathrm{eV}$, respectively. It is also possible to optically excite Ps atoms into Rydberg states and form a Ps beam using electric fields [136], as described in 

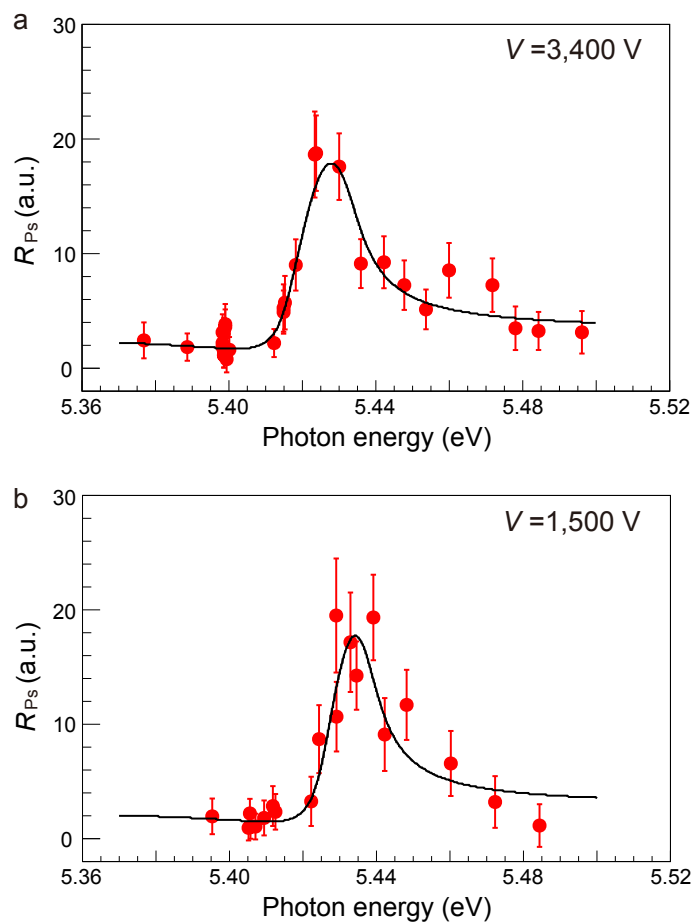

Fig. 37. Ps ion photodetachment signal $\left(R_{\mathrm{Ps}_{\mathrm{s}}}\right)$ as a function of the photon energy for Ps ion acceleration voltages of $3.4 \mathrm{kV}$ (a) and $1.5 \mathrm{kV}$ (b). The solid line is a fit using a Fano profile: [555] convoluted with a Gaussian profile which represents the angular distribution of the Ps ions. The error bars show the standard deviation of the mean signal values including the error of normalization factors. From reference [133].

Section 3.2. This works in the low energy regime $(\leq 1 \mathrm{eV})$, and is most useful for highly excited states. Ps ions can be accelerated to very high energies (10's of keV would not present any real difficulties), but the short lifetime against self-annihilation sets a practical lower limit to obtainable beam energies of a few hundred eV. Ps beams with energies in the keV range can be used as surface probes, for example in grazing angle diffraction studies [349,563]. This methodology may also be used to generate relativistic Ps atoms [564], which would allow for studies of Ps in hitherto unexplored regimes.

\section{2 $\mathrm{Ps}_{2}$ spectroscopy}

Just as with $\mathrm{Ps}^{ \pm}$ions, the Born-Oppenheimer approximation [515] is completely inapplicable to the $\mathrm{Ps}_{2}$ system [565], perhaps maximally so. A wide variety of theoretical techniques have been used to deal with this system (e.g. [566-573]). Shortly after Wheeler's paper was published [16] the stability of $\mathrm{Ps}_{2}$ was demonstrated by Hylleraas and Ore, who obtained a binding energy of $0.11 \mathrm{eV}$ using variational methods [507]. Since then there have been many calculations of the $\mathrm{Ps}_{2}$ binding energy [565,574577]. Recent calculations, which include relativistic corrections, yield $0.4341373 \mathrm{eV}$ [508]. The ground state $\mathrm{Ps}_{2}$ lifetime has also been calculated many times [510,576, $578,579]$, with a recent value of $0.22455(6) \mathrm{ns}[580] . \mathrm{Ps}_{2}$ molecules consist of a pair of oppositely polarized Ps spin configurations. That is, each positron sees one electron in a singlet configuration, and one in a triplet configuration, just as in the Ps ion. Thus, it is not surprising that the $\mathrm{Ps}_{2}$ lifetime is roughly half that of $\mathrm{Ps}^{-}$, because there are two positrons, and therefore twice as many chances to decay at the spin averaged rate.

Despite some superficial similarities to molecular hydrogen [581], $\mathrm{Ps}_{2}$ has completely different properties; the equal mass of all four of its constituents means that the charge distribution of the $\mathrm{P}_{\mathrm{s}_{2}}$ molecule is highly symmetric, and in contrast to the electronic cloud surrounding the slow moving protons of $\mathrm{H}_{2}$, there is no distinction between the motion of electrons and positrons in a $\mathrm{Ps}_{2}$ molecule. One may consider the calculated average interparticle distances to obtain some sense of the geometrical structure of ground state $\mathrm{P}_{2}[508,510,511]$. The positronpositron (electron-electron) distance is on average $\approx 6 a_{0}$, whereas the positron-electron distance is $\approx 4.5 a_{0}$, implying a weakly bound structure comprising two distinct atoms [508], a view supported by the relatively small Ps binding energy (i.e., $0.434 \mathrm{eV}$, as compared to $6.8 \mathrm{eV}$ for Ps atoms). This situation is significantly different from the $\mathrm{H}_{2}$ molecule, which has a proton-proton separation of $\approx 1.5 a_{0}$, and a (covalent) binding energy of $4.52 \mathrm{eV}[171]$. A closer analogue of $\mathrm{Ps}_{2}$ would in fact be the bi-exciton, a bound system with two holes and two electrons in a solid state material [582-584].

Unlike $\mathrm{Ps}^{ \pm}$ions, molecular $\mathrm{Ps}_{2}$ does possess at least one stable excited state, with total angular momentum $L=1$ and negative $\mathrm{C}$ parity [510]. An excited state with $L=0$ and even $\mathrm{C}$ parity has also been predicted $[567,585]$, which suggests the possibility of a Doppler-free two photon excitation of $\mathrm{Ps}_{2}$. This could in principle provide a 1 ppm measurement, limited by the lifetimes of the ground and excited states. We note, however, that the properties of this state are in question because the group representation used in the calculation may not properly take into account the symmetry of the $\mathrm{P}_{2}$ system [569]. Conversely, there can be little doubt as to the existence of the $L=1$ excited state of $\mathrm{Ps}_{2}[510,580,586]$. In fact there is no doubt because this state has been observed [119].

The energy of the excited $\mathrm{Ps}_{2}$ state is $4.94123(2) \mathrm{eV}$ above that of the ground state, corresponding to a transition wavelength of 250.9179(11) nm [580]. The excited state can still decay by annihilation, with a lifetime calculated to be $0.44277(11)$ ns [580]. Note that this is almost twice as long as the ground state $\mathrm{Ps}_{2}$ molecule, and is similar to the Ps ion lifetime. This is what one might expect, since in the excited molecule one of the positron-electron pairs is essentially in an excited $L=1$ state and thus has a negligible decay rate $[184,185]$. This state can decay radiatively to the ground state via an electric dipole transition, and has a radiative lifetime of $1.873 \mathrm{~ns}$, which is almost half the $3.2 \mathrm{~ns}$ lifetime of $2^{3} \mathrm{P}_{\mathrm{J}}$ states of Ps atoms. Thus, radiative decay is in competition with direct annihilation, and the branching ratio $\mathrm{BR}_{2 \mathrm{P}}$ is

$$
\mathrm{BR}_{2 \mathrm{p}}=\frac{(1.873)^{-1}}{(0.44277)^{-1}+(1.873)^{-1}}=19 \%
$$


The fact that the $\mathrm{Ps}_{2}$ excitation frequency to the $L=$ 1 level is fairly close to the $1{ }^{3} \mathrm{~S}_{1} \rightarrow 2{ }^{3} \mathrm{P}_{\mathrm{J}}$ excitation frequency of atomic Ps (the difference is less than $4 \%$ ) also supports the picture of a $\mathrm{Ps}_{2}$ structure formed from two weakly bound Ps atoms. In this case one of the Ps atoms may be excited, with the transition frequency only slightly perturbed by the presence of the other atom, which acts as a dielectric medium [580]. Completely opposed to this view of things are the implications of the permutation symmetries of $\mathrm{Ps}_{2}$ discussed by Schrader [569].

It has been suggested that, since almost $20 \%$ of excited state $\mathrm{Ps}_{2}$ molecules decay by emitting a $251 \mathrm{~nm}$ photon, this may be a useful way to detect their presence [586]. However, this is not likely to be the case in experiments where the same frequency light is used to drive the transition in the first instance, simply because it is likely that pulsed lasers would be required, and the short time-scale and low number of $\mathrm{Ps}_{2}$ molecules would make it extremely difficult to distinguish between the excitation laser light and any light emitted from $\mathrm{P}_{\mathrm{s}_{2}}$ decay. As discussed below, however, excitation and subsequent photoionization of $\mathrm{Ps}_{2}$ along with single-shot lifetime methods have been used to unambiguously detect the formation of $\mathrm{Ps}_{2}$ molecules, and the existence of its $L=1$ excited state [119].

The first experiment in which Ps-Ps interactions were observed [287] was performed by implanting high density positron pulses [356] into porous silica films, such that many Ps atoms were present simultaneously and could interact with each other. We now know that such work was serendipitously assisted by a density enhancement effect caused by Ps atoms preferentially selecting a subset of the available pore volume [290]. These and subsequent experiments $[288,360,587]$ resulted in Ps-Ps scattering events, but could not distinguish between a process known as spin exchange quenching (SEQ) and the formation of $\mathrm{Ps}_{2}$ molecules [287]. SEQ occurs when two oppositely polarized $m= \pm 1$ triplet atoms collide and exchange spins to form two $m=0$ Ps atoms, which can be either singlets or triplets, with both processes having similar cross sections $[588,589]$. In a strong magnetic field the $m=0$ triplet states will have a short lifetime and the singlet states will also decay rapidly, meaning that SEQ converts long-lived atoms into short lived atoms. $\mathrm{Ps}_{2}$ formation effectively converts two oppositely polarized long-lived Ps atoms into two short-lived atoms, also increasing the overall decay rate.

The signal in the Ps-Ps scattering experiments was a density-dependent change in the Ps decay rate, measured via single-shot lifetime methods [288]. This approach provides a very clear signal (see Fig. 38), and can even be used to determine the spin polarization of the incident positron beam [362]. This is possible because CP violation in beta decay means that positrons produced from ${ }^{22} \mathrm{Na}$ sources are intrinsically spin polarized [590,591]. This polarization is not lost in moderation [592] or trapping processes [362], and carries over to the Ps, meaning that there will be an asymmetry in the fraction of $m= \pm 1$ states produced. Increasing the Ps density to the point where Ps-Ps scattering can occur will lead to losses via SEQ or $\mathrm{Ps}_{2}$ molecule formation, but eventually the minority spin component will be exhausted, after which there will be
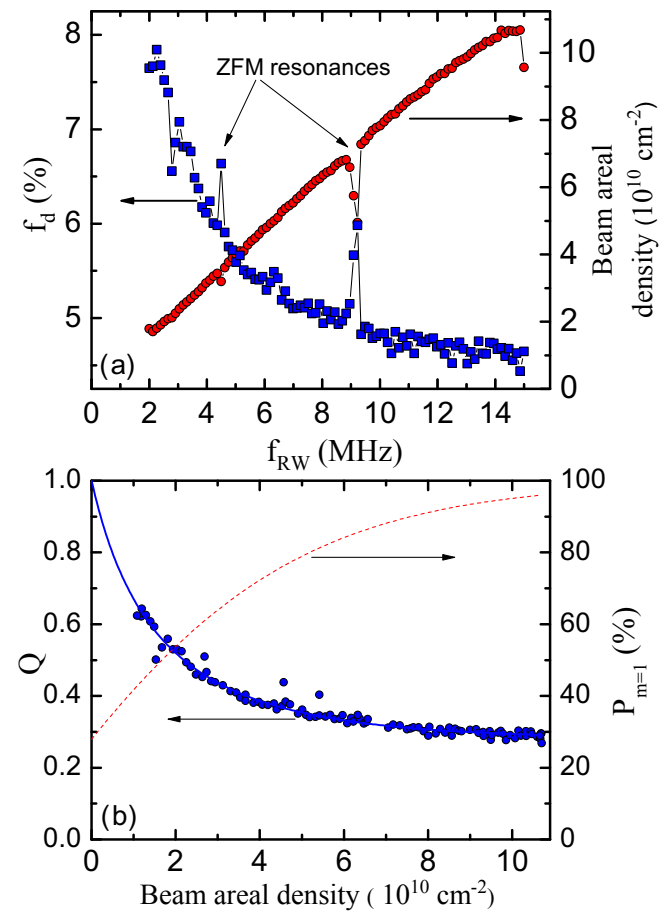

Fig. 38. (a) Delayed fraction $\left(f_{d}\right)$ and positron beam areal density measured as a function of the rotating wall (RW) compression frequency in a $2.3 \mathrm{~T}$ magnetic field. These data show the linear positron beam density dependence as a function of the RW frequency, indicative of strong-drive compression $[354,593]$ and the non-linear reduction in the delayed fraction, resulting from the initial positron beam spin polarization. Also indicated are the zero-frequency mode (ZFM) resonances that cause the beam to abruptly heat up and expand, lowering the density [355]. (b) Normalized delayed fraction (Q) as a function of the beam energy. $\mathrm{Q}$ may be used to calculate the $m=1$ Ps spin polarization, indicated by the dashed line. The solid line is a fit to a model that includes a density-dependent PsPs induced decay rate. The constant value of $\mathrm{Q}$ approached at high densities arises when the minority spin component has been depleted, and the Ps gas is almost entirely spin polarized. From reference [362].

no further density dependent changes in the decay rate. This is evident from the data shown in Figure 38, which show how the delayed fraction (see Sect. 3.1) changes with increasing Ps density via this process. At the highest density, where only one Ps spin-state remains [362], increasing the density further has no effect. Since this happens only when one spin state has been fully depleted, these data can be used to estimate the incident positron beam spin polarization, yielding $\approx 30 \%$.

While the quenching data provide incontrovertible evidence for Ps-Ps interactions, the time resolution of the SSPALS technique cannot distinguish between SEQ and $\mathrm{Ps}_{2}$ formation. Since $\mathrm{Ps}_{2}$ formation requires a third body to conserve energy and momentum, some arguments can be made relating the presence of Ps surface states, to the production of $\mathrm{Ps}_{2}[288,360]$. However, such arguments require a detailed understanding of Ps dynamics. For example, in mesoporous films, in which the first $\mathrm{P}_{2}$ signal 


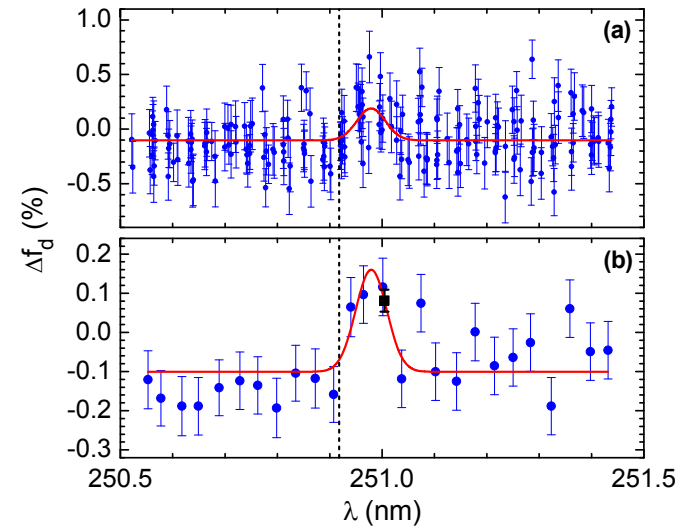

Fig. 39. $\mathrm{P}_{2}$ excitation signal measured using high density Ps produced in a mesoporous silica film. The raw data from four runs (a) have been re-binned (b) to show the resonance more clearly. The vertical scale is the change in the delayed fraction in percent. The measurement for the single square point in (b) was obtained in a single long run, as described in the text. The solid lines are Gaussian fits to the data from which we determine a line center of $\lambda=250.979 \pm 0.006 \mathrm{~nm}$ and a width of $0.069 \pm 0.026 \mathrm{~nm}$ (FWHM). The dashed vertical line indicates the calculated vacuum resonant wavelength for the $\mathrm{Ps}_{2}$ excitation. From reference [119].

was thought to have been obtained [360], Ps atoms may have significant interactions with the walls, owing to their long de Broglie wavelength, even in the absence of any Ps surface states. These kinds of experiments therefore necessarily constitute indirect observations. The optical excitation of $\mathrm{Ps}_{2}$, on the other hand, is completely unambiguous and constitutes a direct observation of the $\mathrm{P}_{\mathrm{S}_{2}}$ system, and confirms the existence of its first excited state [119].

Two measurements have been performed which show optical excitation of $\mathrm{Ps}_{2}$ [119]. In one experiment a porous silica target was utilized, as shown in Figure 39, and in the other $\mathrm{Ps}_{2}$ was generated on an $\mathrm{Al}(111)$ surface, as indicated in Figure 40. In both cases a high-density positron pulse was obtained using a pulsed $2.3 \mathrm{~T}$ magnetic field [356] and plasma manipulation techniques [593]. The positron beam densities required to obtain Ps densities sufficient to observe Ps-Ps interactions (and thus also $\mathrm{P}_{\mathrm{S}_{2}}$ formation) can be estimated from calculated $\mathrm{P}_{\mathrm{S}}-\mathrm{Ps}_{\mathrm{S}}$ scattering cross sections $\sigma_{\mathrm{Ps}}=1 \times 10^{-15} \mathrm{~cm}^{-2}$ [589]. The scattering rate for a Ps density $\rho$ will then be $\Gamma=\rho \sigma_{\mathrm{Ps}} v$, where we assume thermal Ps speeds $\left(v=10^{7} \mathrm{~cm} \mathrm{~s}^{-1}\right)$. For Ps atoms produced in porous silica targets the emission rate into vacuum (where the density will be significantly reduced) is expected to be on the order of $100 \mathrm{MHz}$ [126], and thus we might expect that the Ps-Ps scattering rate must be similar to this in order for such interactions to take place; this implies Ps densities of $10^{16} \mathrm{~cm}^{-3}$.

For positrons implanted into porous silica targets the resulting Ps density can be estimated using Mahkovian stopping profiles [306,307], and by considering Ps emission rates [126]. Typical parameters in the high-density experiments $[288,290,362]$ were a stopping distance of $\approx 100 \mathrm{~nm}$, and a beam areal density up to $10 \times 10^{10} \mathrm{~cm} \mathrm{~s}^{-1}$, leading to instantaneous Ps densities approaching the required

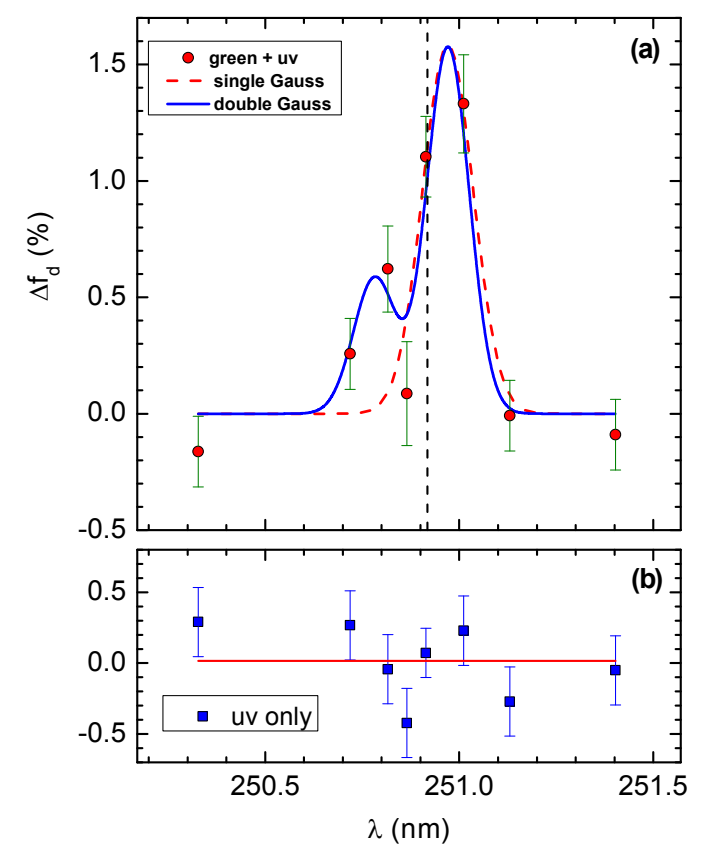

Fig. 40. $\mathrm{Ps}_{2}$ excitation conducted in vacuum with an $\mathrm{Al}(111)$ crystal target: (a) lineshape measured with both the UV and green lasers on. The dashed curve is a single Gaussian fit to the data and the solid line is a Double Gaussian fit constrained to have only one width. The dashed vertical line indicates the theoretical resonant wavelength for the $\mathrm{Ps}_{2}$ excitation in vacuum. (b) Measurement with only the UV laser, showing the best fitting horizontal line, $\Delta f_{d}=0.016 \pm 0.087$, consistent with no effect. From reference [119].

$10^{16} \mathrm{~cm}^{-3}$. Moreover, we expect that the quantum confinement effects may further increase the Ps density in such materials, facilitating Ps-Ps interactions [290]. Ps and $\mathrm{Ps}_{2}$ dynamics are much more complicated on a metal surface [594], and such simple rate estimates are likely meaningless, especially since the surface conditions may well be very unstable on the time scale of $\mathrm{Ps}_{2}$ experiments [587]. We can, however, expect the density requirements in this case to be much more stringent, owing to the relatively short lifetime of positrons on metal surfaces [270].

After $\mathrm{Ps}_{2}$ formation pulsed lasers were used to excite the optical transition; the detection scheme involved excitation and subsequent photoionization of $\mathrm{P}_{\mathrm{S}_{2}}$ in the same way as has been used for Ps excitation experiments. In this case the signal would be an increase in the mean Ps lifetime, since in some cases ground-state triplet Ps atoms would be produced. However, this is not the only possibility, as $\mathrm{Ps}_{2}$ photoionization can also result in the production of a Ps ion (positive or negative) which would not provide a detectable signal. That is, the following processes may occur when excited states of $\mathrm{Ps}_{2}$ are photoionized:

$$
\begin{gathered}
\mathrm{Ps}_{2}^{*}+h \nu \rightarrow \mathrm{Ps}^{ \pm}+e^{\mp} \\
\mathrm{Ps}_{2}^{*}+h \nu \rightarrow \operatorname{Ps}+e^{+}+e^{-} .
\end{gathered}
$$

These processes have energy thresholds of 1.96 and $2.1 \mathrm{eV}$ respectively. In the experiments however green light of 
wavelength $532 \mathrm{~nm}$ was used which corresponds to an energy of $2.3 \mathrm{eV}$ and can thus drive both processes. While it would have been interesting to try to resolve the mechanisms the delayed fraction signal was not sufficient to perform such measurements [119]. Half of all $\mathrm{Ps}_{2}$ photoexcitation events would result in the production of Ps ions or ground state singlet atoms. The free positrons would likely annihilate rapidly as well, meaning that for equal production of all outcomes shown above only $50 \%$ would actually produce a signal (assuming that no Ps ions were subsequently photoionized). Thus, the low number of $\mathrm{Ps}_{2}$ molecules produced is exacerbated by limitations in possible detection. The practical difficulties associated with the optical excitation of $\mathrm{Ps}_{2}$ are, unfortunately, all too evident from the data of Figures 39 and 40.

$\mathrm{Ps}_{2}$ formed in the voids of porous silica would be unlikely to escape before annihilation, meaning that optical excitation must also occur inside the material. These experiments are therefore similar to those conducted with (lower-density) Ps atoms confined in a silica film [115] (see Sect. 3.1.3). In that work a cavity induced shift in the line center was observed, shifting to shorter wavelengths. Although the exact shift of the energy levels of confined Ps atoms is difficult to calculate [403,407], they would be expected to increase, and the observed shift is therefore qualitatively unsurprising. For the $\mathrm{Ps}_{2}$ case a cavity shift in the resonant wavelength was also observed [115], as shown in Figure 39. However, in this case the observed shift appeared to be to longer wavelengths. This observation is not currently understood: the existing data is not sufficient to formulate any detailed hypothesis and more measurements are required.

The lineshape observed from excitation of $\mathrm{Ps}_{2}$ formation on an $\mathrm{Al}(111)$ surface (Fig. 40) appears to be consistent with the theoretical value. However, these data were recorded with an unstable target (see [119] for details) and so the statistics required for an accurate lineshape could not be obtained. Moreover, the possibility that laser light reflected from the crystal surface would give rise to two peaks, one blue and one red shifted, means that the line center determination is considerably less precise. This problem is unavoidable given that $\mathrm{Ps}_{2}$ is unlikely to travel more than a few microns from the surface upon which it is produced, although a thin metal transmission target might allow one to measure the red and blue shifted components independently in order to evaluate the true line center.

Both of the previous experiments can be interpreted as highly significant with regard to the existence of $\mathrm{Ps}_{2}$ and its excited state, and additional work is certainly warranted in this area. Experimental improvements would be required to measure the decay rate of $\mathrm{Ps}_{2}$, but improvements in the optical spectroscopy do seem to be obtainable using similar techniques. A remoderated positron beam with a higher density, the identification of more stable $\mathrm{Ps}_{2}$ formation targets, and direct detection of photoionization products would all provide improvements, and may facilitate ppm spectroscopy of the $L=1 \mathrm{Ps}_{2}$ excited state. If it exists, the $L=0$ excited state [585] could also be used to improve the signal since Doppler free methods might then be employed [494,495].

\section{Future directions of Ps-laser physics}

Positronium laser spectroscopy has advanced rapidly in recent years, largely because of the advent of Surko traps [69] (see Tab. 1), and we can expect many more new experiments to be conducted in the near future. In this concluding section I consider briefly a few examples of next-generation Ps experiments that I believe have now become more feasible; some of these are probably happening already. For example, significant progress has been made in Stark manipulation techniques, (e.g. $[136,141])$, suggesting that Stark deceleration and trapping of Rydberg Ps is on the horizon. This is highly likely to result in the production of focused and slow Ps beams, which have direct applications to new Ps spectroscopy and studies of Ps interactions with other atoms and molecules. They are also expected to be a key aspect of possible gravity measurements [477]. New ways of generating cold Ps (including engineered mesoporous materials) should improve high-density Ps work, allowing for improvements to $\mathrm{Ps}^{-}$and $\mathrm{Ps}_{2}$ and molecule spectroscopy experiments, and to progress towards the creation of a Ps BEC [595].

\subsection{Stark deceleration and trapping}

The experiments conducted so far in which Ps atoms have been guided by electrostatic fields (see Sect. 3.2.2) are only the first step in the manipulation of Rydberg states using inhomogeneous electric fields. Longer term plans are being implemented which seek to replicate some of the experiments that have already been conducted in this area (e.g. [338,436,454-456,459,462-464,469]).

As discussed in Section 3.2.2, if a Rydberg atom with a positive Stark-shift (see Fig. 6) enters an electric field it will slow down, since kinetic energy will be transferred into the Stark energy. If the electric field is then switched off (adiabatically) before the atom leaves it will experience a net reduction in its kinetic energy. That is to say, the atom will have been decelerated. If this is done many times the atom may even be trapped. This technique has been demonstrated with polar molecules [447,448,596], and is even more effective with atoms or molecules excited to appropriate Rydberg states, as their dipole moments may be extremely large [338].

The ability to decelerate Ps atoms will be useful because all of the physical processes through which Ps atoms are usually produced (see Sect. 2.2.1 result in the production of thermal (or hotter) Ps atoms. This means that they have speeds of $\sim 7 \times 10^{4} \mathrm{~m} \mathrm{~s}^{-1}$, much faster than one can obtain from a supersonic gas expansion (e.g. [597]) or a buffer gas source (e.g. [598]). In addition to their fast mean speeds, the broad speed distributions of Ps atoms can also present some practical difficulties when it comes to designing suitable electrode structures that are capable of capturing a large fraction of the available atoms. Time-varying methods may be required to deal with the distributions in situations where static fields would otherwise be suitable [599]. Techniques that ordinarily employ time-varying fields (such as deceleration [456]) may be impossible, or may only be applied to a small part of the available Ps ensemble. As discussed in Section 3.1, 

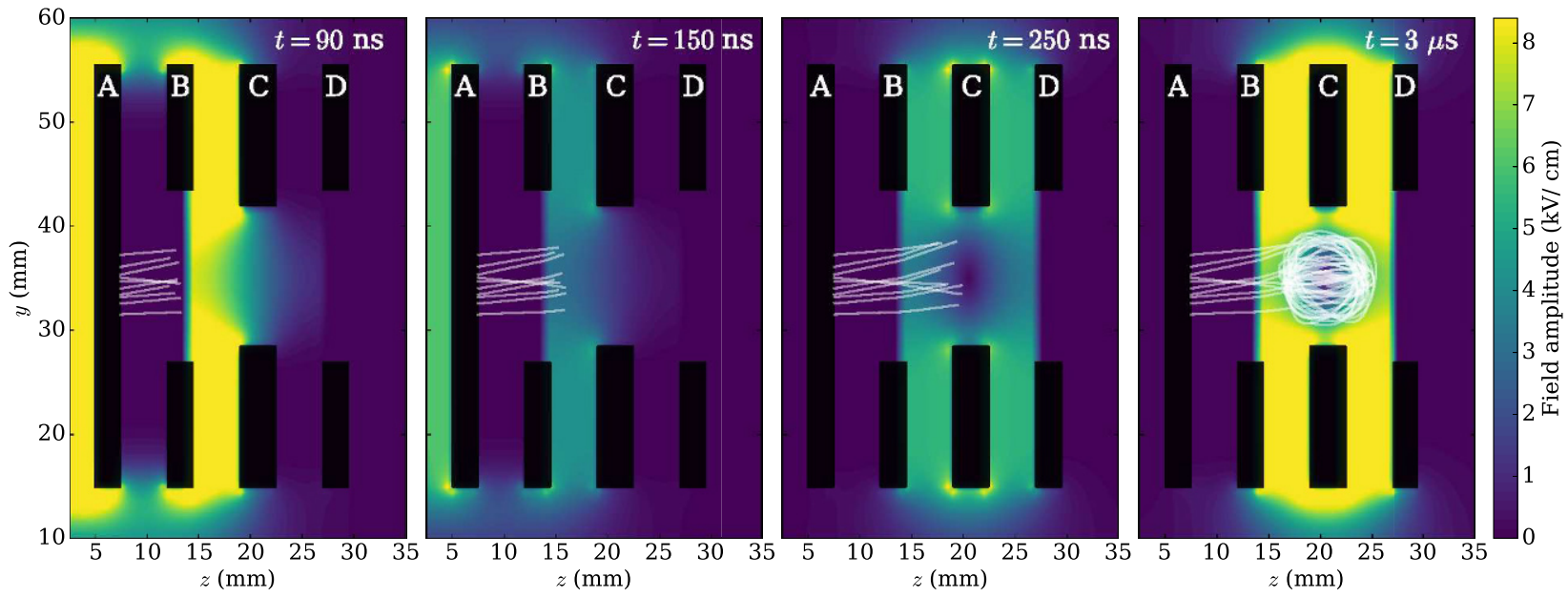

Fig. 41. Simulated trajectories of 10 Ps atoms $(n=13, k=+11)$ with longitudinal kinetic energies of $\sim 33$ meV. The black bars represent cross sections of various electrodes: a is also the Ps production target, B and D are electrodes with a high transmission circular mesh and $\mathrm{C}$ is a $3 \mathrm{~mm}$ thick square electrode with a $13 \mathrm{~mm}$ hole in the center which forms the center of the trap. The panels indicate the fields applied at different times after laser excitation, as indicated. The white lines denote the trajectories of each atom since excitation, and the color scale represents the electric field strength, with the scale truncated at $8.3 \mathrm{kV} \mathrm{cm}^{-1}$, where the ionization rate exceeds $10^{8} \mathrm{~s}^{-1}$

Doppler broadening is also a limiting factor in many Ps spectroscopic experiments.

However, the underlying principle behind all Starkbased manipulation techniques is that it is the energy shifts of atoms in electric fields that matters. Although they are fast moving, Ps atoms do not possess large amounts of energy: $25-100 \mathrm{meV}$ is quite typical. The atomic structure of Ps is similar enough to that of other atoms that achieving the necessary Stark shifts to allow application of standard deceleration techniques does not require particularly large electric fields. The main difficulty is simply in applying the appropriate fields in a manner that is consistent with the Ps speed and angular distributions. If this can be achieved, the alreadydemonstrated methodology should be readily applicable. Furthermore, once Ps atoms are accepted into any suitable device their low mass should allow large accelerations to be applied, and it may be the case that controlling Ps atoms in this way is extremely effective.

In order to determine the feasibility of decelerating and trapping Rydberg positronium we performed a series of trajectory simulations. These simulations are based upon those used to model Ps guiding (see Sect. 3.2.2) and so we can expect that they will provide a reasonably accurate representation of our real experimental conditions. This will then make it possible to refine a design for the electrode structure in a Rydberg Ps trap. Figure 41 shows the results of the simulation at four different times following Rydberg Ps production. A series of plate electrodes with central holes (shown in black in the figure) are used to define electric field gradients close to a Ps formation target. The trap is configured such that a central $13 \mathrm{~mm}$ hole in electrode $\mathrm{C}$ is the trap center, where atoms will oscillate around an equilibrium position. The voltage applied to all four electrodes must be controlled independently during each time-step in the simulation to generate the indicated electric fields.

The simulation includes trajectories of 5000 Ps atoms, generated using Monte-Carlo methods, that determine the initial velocity spread using a Maxwell Boltzmann distribution. The actual velocity distribution is unknown, and in previous simulations MB distributions were used as an approximation, with the temperature arbitrarily adjusted to improve agreement with measurements [130]. The distribution in this case was truncated to account for the lower-limit of Ps emitted from mesoporous films owing to the confinement energy [109].

The results of the simulation indicate that both deceleration and trapping will occur for some of the slowest atoms. In the between the first and second panels of Figure 41 the voltage applied to electrodes A and B is decreased from $5.5 \mathrm{kV}$ to $0 \mathrm{~V}$, and then the voltage applied to $\mathrm{C}$ is increased from $0 \mathrm{~V}$ to $5.5 \mathrm{kV}$, forming the electric field minimum seen in the last two panels, the voltage applied to D is always $0 \mathrm{~V}$. The time scales for the simulated HV switching are experimentally achievable [139].

The simulations indicate that we should be able to extract $\sim 12 \mathrm{meV}$ of energy from most of the atoms, leading to a trapping efficiency of $\sim 3.0 \pm 0.2 \%$. We found that for our experimental limitations (including voltage switching times, maximum switching voltage, and Ps distribution), $n=13$ was the optimal state to trap; higher-lying states have lower ionization energies, and thus will always have less energy extracted before loss, and lower-lying states require stronger electric fields, and have significantly lower fluorescence lifetimes [134].

A proof-of-principle Ps trapping experiment is likely to occur relatively soon, and should clarify the nature of any problems overlooked by the simulations. This in turn will allow improved simulations and inform new 
Table 6. State of the art precision optical and microwave measurements of $\mathrm{Ps} 1{ }^{3} \mathrm{~S}_{1} \rightarrow 2{ }^{3} \mathrm{~S}_{1}$ interval, ground state hyperfine interval, and $n=2$ fine structure. Both the theoretical and experimental uncertainty $(U)$ is shown for each transition. Only directly measured intervals are included (i.e., those that can be inferred from other pairs of measurements are not listed). The theoretical uncertainties are all from reference [150], except the ground state hyperfine interval, which is from reference [604]. Two experimental measurements of $E_{\mathrm{hfs}}$ are given because the recent work by Ishida et al. [81] uses an energy selection method that is expected to remove possible errors related to nonthermalized Ps [81], although currently the uncertainty is too large to determine if this is in fact the source of the discrepancy between previous experiments and theory.

\begin{tabular}{llllll}
\hline Transition & Experimental result $(\mathrm{MHz})$ & $U(\exp )$ & Year & $U$ (theory) & Ref. \\
\hline $1{ }^{3} \mathrm{~S}_{1} \rightarrow 2^{3} \mathrm{~S}_{1}$ & $1233607216.4 \pm 3.2$ & $2.6 \mathrm{ppb}$ & 1993 & $0.8 \mathrm{ppb}$ & {$[107]$} \\
$1^{3} \mathrm{~S}_{1} \rightarrow 1^{1} \mathrm{~S}_{0}$ & $203389.10 \pm 0.74$ & $3.6 \mathrm{ppm}$ & 1984 & $2.5 \mathrm{ppm}$ & {$[377]$} \\
$1^{3} \mathrm{~S}_{1} \rightarrow 1{ }^{1} \mathrm{~S}_{0}$ & $203394.2 \pm 1.6_{\text {stat }} \pm 1.3_{\text {sys }}$ & $10.1 \mathrm{ppm}$ & 2014 & $2.5 \mathrm{ppm}$ & {$[81]$} \\
$2^{3} \mathrm{~S}_{1} \rightarrow 2^{3} \mathrm{P}_{0}$ & $18499.65 \pm 1.2_{\text {stat }} \pm 4.0_{\text {sys }}$ & $226 \mathrm{ppm}$ & 1993 & $7.0 \mathrm{ppm}$ & {$[266]$} \\
$2^{3} \mathrm{~S}_{1} \rightarrow 2^{3} \mathrm{P}_{1}$ & $13012.42 \pm 0.67_{\text {stat }} \pm 1.54_{\text {sys }}$ & $129 \mathrm{ppm}$ & 1993 & $10.0 \mathrm{ppm}$ & {$[266]$} \\
$2^{3} \mathrm{~S}_{1} \rightarrow 2^{3} \mathrm{P}_{2}$ & $8624.38 \pm 0.54_{\text {stat }} \pm 1.40_{\text {sys }}$ & $174 \mathrm{ppm}$ & 1993 & $15.1 \mathrm{ppm}$ & {$[266]$} \\
\hline
\end{tabular}

designs for decelerators and traps suitable for Ps. However, trapping a barely detectable number of Ps atoms is not in itself particularly useful. It will be essential to find ways to perform Ps deceleration with high efficiency so that further experiments can be realized using these techniques. Some specialty experiments, in which low numbers of atoms are acceptable, or even necessary, may benefit from experiments similar to the simulation described above. The physics of trapping Ps atoms is practically identical to that of other Rydberg systems, and therefore there is little reason to study the trapping in this way (assuming that there are no interesting effects related to the low Ps mass). In all likelihood, the ultimate utility of Ps deceleration experiments will be coupled also to developments in producing colder Ps atoms.

\subsection{Precision spectroscopy}

One of the motivations driving Ps experimentation, particularly spectroscopic $[103,107,228,264,322,377]$ and lifetime $[82,83,163,195]$ measurements, is that, as a system composed of two low-mass leptons, Ps is an ideal testing ground for QED theory [144]. Precision measurements performed using a wide variety of both hydrogenic and non-hydrogenic atoms, ions, and molecules are, of course, also used for QED tests (e.g. [600-603]), but Ps has some unique properties in this regard, since it has no constituents with internal structure, and the equal mass of the electron and positron mean that it is very sensitive to recoil effects [146]. All QED corrections to Ps energy levels are now known up to order $\mathrm{m} \alpha^{6}$ [150-152], and some of the higher order terms have also been calculated (e.g. $[153,154])$. Recent theoretical developments are summarized in reference [155].

All calculated Ps energy levels and intervals are more accurately known than measured values, as indicated in Table 6. This table includes microwave and (one) precision optical measurement [107]. In general one can expect optical measurements to be considerably more precise than those obtained from microwave spectroscopy, but it is nevertheless instructive to consider how well the energy levels of Ps are known experimentally (see also [172]).
As described in Section 3.3, the $1{ }^{3} \mathrm{~S}_{1} \rightarrow 2{ }^{3} \mathrm{~S}_{1}$ transition has been measured with an experimental uncertainty of $\pm 3.2 \mathrm{MHz}$; this measurement is now 25 years old. There are two new experiments in progress seeking to improve the precision of this energy interval $[76,504]$. It is thought that improvements on the previous work may be possible since optical metrology is now much more advanced, and frequency calibration at a level orders of magnitude better than necessary can be obtained with commercially available frequency combs. Moreover, excitation of $2^{3} \mathrm{~S}_{1}$ Ps atoms to Rydberg levels and subsequent detection will allow trajectory and speed mapping to be incorporated in the $1^{3} \mathrm{~S}_{1} \rightarrow 2{ }^{3} \mathrm{~S}_{1}$ lineshape model. No new results have yet been reported.

The ground state hyperfine interval has been measured with an uncertainty comparable to the theoretical uncertainty, although there is a persistent discrepancy amounting to $\approx 3 \sigma[228,264,322,377]$. This is widely believed to be related to the need to extrapolate data at high gas pressures to zero pressure ot account for a pressure shift. The linear extrapolation used is evidently not sufficient and so Ps thermalization effects need to be explicitly measured or accounted for in a different way. This is similar to situation in several measurements of the o-Ps decay rate, which have now been resolved $[82,83]$. New hyperfine interval measurements using the same technique, but with time-selection to remove signal from non-thermal Ps, are underway [81]. The data so far are consistent with theory, but time-selection severely reduces the amount of data that can be obtained. A measurement with sufficient uncertainty to potentially resolve the problem is expected to be completed by 2020 . Some different experimental methods, that do not suffer from pressure shifts, have been demonstrated $[121,381]$ but they are unlikely to be able to reach the required precision in the foreseeable future. It may also be possible to directly measure the singlet-triplet splitting in the $n=2$ manifold to address this discrepancy.

Measuring the Lamb shift [605] in Ps is, in some sense, less interesting than it is in hydrogen because the $\mathrm{S}$ and $\mathrm{P}$ degeneracy is already lifted by annihilation and spinspin interactions. However, there are other QED effects that can be studied via $n=2$ spectroscopy. An ultra fine 


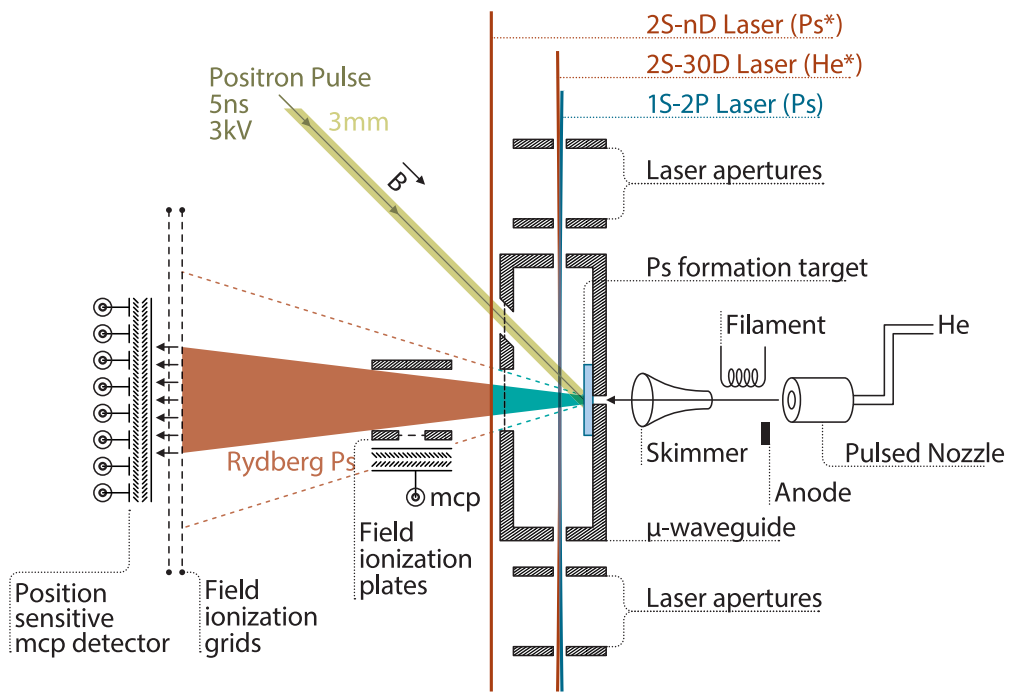

Fig. 42. Schematic layout of a microwave spectroscopy experiment. A positron pulse is implanted into a Ps formation target located inside a waveguide. Atoms are excited from $2{ }^{3} \mathrm{P}_{\mathrm{J}}$ levels to metastable $2{ }^{3} \mathrm{~S}_{1}$ levels via microwave transitions. The longerlived $2{ }^{3} \mathrm{~S}_{1}$ atoms are able to leave the waveguide, where they are excited optically to Rydberg levels and subsequently detected with either a position sensitive MCP or by pulsed field ionization, so as to increase the detection efficiency and to provide trajectory information to correct for Doppler effects. Electric and magnetic fields in the excitation region will be mapped out by performing high-resolution spectroscopy on He atoms produced from a supersonic expansion. A similar arrangement can be used to excite Rydberg Ps levels in a waveguide and perform microwave spectroscopy between nearby Rydberg states.

$(\approx 75 \mathrm{kHz}$ for $n=2)$ splitting in positronium between the $L=1$ singlet state and the spin average of the triplet states has recently been shown to arise at order $\alpha^{6}[606]$. Measurements of this splitting at sufficiently high precision can constrain some proposed ultralight interactions, at a level that depends somewhat on the model being tested, but that would likely only become of interest at the sub $100 \mathrm{kHz}$ level. It may even be possible to perform an experimental test of the noncommutativity of space in this way [607].

The present $\approx \mathrm{MHz}$ uncertainties in the $n=2$ fine structure measurements [264-266] can almost certainly be reduced. One of the limiting factors in previous experiments was that $n=2$ atoms had to be produced through positron collisions with certain surfaces [262,263,608], which is very inefficient (on the order of $0.1 \%$ ), and thus imposed statistical limitations. Much more efficient production of $n=2$ atoms can be accomplished by laser excitation [139]. Other improvements that can be made to Ps fine structure microwave spectroscopy involve exciting atoms to Rydberg levels for trajectory and time mapping, to reduce or eliminate Doppler effects [122], or one could set up the microwave radiation in a standing wave to drive two-photon transitions, tuning through the resonance via the Stark effect.

A schematic layout of a new experiment ${ }^{1}$ currently underway at UCL for Ps microwave spectroscopy is shown in Figure 42. This arrangement will involve Ps production in a suitable waveguide where laser excitation can take place. It will also incorporate a metastable He beam that will be used to measure electric and magnetic fields

\footnotetext{
${ }^{1}$ This experiment is based on a scheme first outlined by Allen Mills.
}

by optical He spectroscopy [420]. There are several different possible techniques that can be used with this general arrangement. For example, in some versions short lived $2^{3} \mathrm{P}_{\mathrm{J}}$ states will be excited to longer lived $2^{3} \mathrm{~S}_{1}$ states by microwaves, and will then be able to travel out of the waveguide structure and be excited to Rydberg levels. This will provide an improved signal to noise ratio, and allow for trajectory mapping. However, it will also be possible to produce $2^{3} \mathrm{~S}_{1}$ atoms directly by laser excitation [139], and then to drive microwave transitions to the shorter lived $2^{3} \mathrm{P}_{\mathrm{J}}$ levels. This method has the advantage that lower microwave power would be required, since the $2^{3} \mathrm{~S}_{1}$ lifetimes allow longer irradiation times. However, it has the disadvantage that the $2^{3} \mathrm{~S}_{1}$ production rate would be relatively low. Various different approaches will be evaluated to determine which systematics are dominant in measurements at the $100 \mathrm{kHz}$ level, and thus how the experiment can be improved beyond this. Ultimately the $\approx 50 \mathrm{MHz}$ natural linewidth of such measurements (owing to the $3.2 \mathrm{~ns} 2^{3} \mathrm{P}_{\mathrm{J}}$ radiative lifetimes) will be the limiting factor, meaning that it would be necessary to split the line by a factor of 1000 to obtain a precision of $50 \mathrm{kHz}$, regardless of any other statistical or systematic limitations.

The second phase of the UCL spectroscopy program seeks to perform measurements of transitions between Rydberg states, with the ultimate aim of measuring the Rydberg constant. The initial experiments will comprise transitions between $n$ states (around $n \approx 25$, with $\Delta n=2$ ). These transitions occur at microwave frequencies on the order of $100 \mathrm{GHz}$, and initial measurements with uncertainties on the order of $100 \mathrm{kHz}$ (i.e., $1 \mathrm{ppm}$ ) are anticipated. Based on preliminary measurements using Rydberg He, however, we expect eventually to be able to 
improve this to the $1 \mathrm{kHz}$ level (i.e., $10 \mathrm{ppb}$ ). Helium test experiments have shown that, in order to reach this level of precision (or better), it will be necessary to develop external electric field cancellation techniques at the $\mathrm{mVcm}^{-1}$ level. This will be done using He atoms excited to Rydberg levels to measure the fields [160] as indicated in Figure 42.

A similar microwave measurement of the Rydberg constant in hydrogen has been performed [609], but the results were not published, apparently because of unresolved uncertainties regarding the effects of external fields. This is a difficult problem to solve when dealing with atoms in Rydberg states, but Ps offers one advantage in this regard: circular states can be generated that would be less sensitive to external electric and magnetic fields, because Ps has no net magnetic moment in the triplet state. This is because the electron and positron magnetic moments are equal and opposite. Thus, circular states of Rydberg Ps should be an ideal system to for precision spectroscopic measurements even if external fields cannot be completely eliminated.

These measurements will make it possible to evaluate in detail the experimental systematics (including field cancellation) that limit the obtainable precision, in preparation for the third phase of the program, whose aim is to perform optical transitions between Rydberg and $n=2$ levels. These are chosen because the linewidth of such transitions will be narrower than the corresponding transitions to the ground state, owing to the longer lifetime of the radiatively metastable $n=2$ level (i.e., 8 times longer): this would result in a $\approx 100 \mathrm{kHz}$ linewidth. Although many parameters need to be measured before realistic estimates can be made, it seems reasonable to expect that this line could be split by at least a factor of 100, giving a ppt measurement. If it does become possible to measure the Rydberg constant with Ps to a part in $10^{12}$, doing so will certainly be very challenging. One may well ask why it is worthwhile to attempt such a measurement when there already exist much more precise measurements, obtained from various other systems, especially hydrogen [610]. This is a very reasonable question, and the answer is related to what has become known as the "proton radius puzzle" $[157,159]$.

Experiments with atomic hydrogen have driven developments in precision spectroscopy essentially since lasers were invented (e.g. [611]), and such measurements have now reached astounding levels of precision, with the benchmark $1{ }^{3} \mathrm{~S} \rightarrow 2{ }^{3} \mathrm{~S}$ transition measured with a fractional uncertainty of $4.2 \times 10^{-15}$ [491]. At this level of precision measurements become sensitive to very small effects that might otherwise have no relevance. Moreover, interpreting such data requires a complementary theoretical effort at an even higher precision. In the case of hydrogen such descriptions are hindered by incomplete knowledge of the proton charge radius. Hydrogen energy levels are affected by the finite size of the proton charge distribution, but since this is determined by Strong Force interactions between quarks, the levels cannot be calculated with high precision. Because of this, precision spectroscopy of hydrogen requires two measurements, essentially to measure the proton charge radius (or, equivalently, the Lamb shift), and the Rydberg constant separately. Despite the very precise $1{ }^{3} \mathrm{~S} \rightarrow 2{ }^{3} \mathrm{~S}$ measurements, the additional, less precise measurements that must also be performed limit the extent to which a complete description of the hydrogen atom can be obtained.

In order to improve this situation an experiment was conducted in 2010 in which spectroscopy of muonic hydrogen (that is, the bound state between a proton and a negative muon [612]) was used to make a high-precision determination of the proton charge radius [157]. Because muons are almost 200 times heavier than electrons, they will have a stronger interaction with the proton in a hydrogenic system, and this leads to a much larger sensitivity to the proton charge radius. The relative wave function overlap will be approximately the muon/electron mass ratio cubed (i.e., $\approx 200^{3}=8 \times 10^{6}$; see also [613] for a summary of the theory of the Lamb shift in muonic hydrogen). Note that, since they have a non-zero wavefunction overlap at the origin, only S states are sensitive to the proton charge radius, in much the same way that only $\mathrm{S}$ states of Ps annihilate (excluding higher order contributions).

Since muonic hydrogen is nearly 10 million times more sensitive to the proton charge radius than electronic hydrogen, it is possible to obtain a very precise measurement the former, even with relatively crude spectroscopic methods. The goal of muonic hydrogen experiments was to obtain a highly accurate proton charge radius number, which would then allow the Rydberg constant to be extracted from precision spectroscopic measurements of atomic hydrogen more accurately. However, the measurement [157] revealed a significant discrepancy with the expected proton radius, which was known not only from hydrogen spectroscopy but also from electron-proton scattering measurements (see Fig. 43), and between which there was no disagreement [159]. Subsequent experiments have only made the problem worse $[158,616]$, and this discrepancy remains unexplained, despite an enormous amount of theoretical effort $[617,618]$.

The measurements that, taken in conjunction with the precision $1 \mathrm{~S}-2 \mathrm{~S}$ data [491], give the proton radius are shown in Figure 43 [614]. These include microwave Lamb shift [605] measurements [619,620] performed using the separated oscillatory field method of Ramsay [621], as well as optical $2 \mathrm{~S}-n \mathrm{~S} / \mathrm{D}$ transitions in hydrogen $[161,162,610$, 622-624]) (for some reason, always with even values of $n$ ). It is clear from this figure that the spread of all the individual measurements that go into the final value is considerably larger than the apparent eventual discrepancy, which implies that even if a small systematic error in these measurements exists, it could be sufficient to resolve the puzzle.

Recently a measurement of the $2 \mathrm{~S}-4 \mathrm{P}$ transition in hydrogen [614] has revealed the existence of a possible systematic shift that may mean that the Rydberg constant derived from hydrogen spectroscopy is the source of the discrepancy, as indicated in Figure 43. However, more measurements are required to verify if this is really the case. Despite this intriguing result, the situation is far from resolved, and there are currently several experimental programs underway that will address the problem using a variety of systems [159]. Ps spectroscopy is just one of these, and while it is unlikely to be able to compete 
Proton charge radius $r_{\mathrm{p}}(\mathrm{fm})$

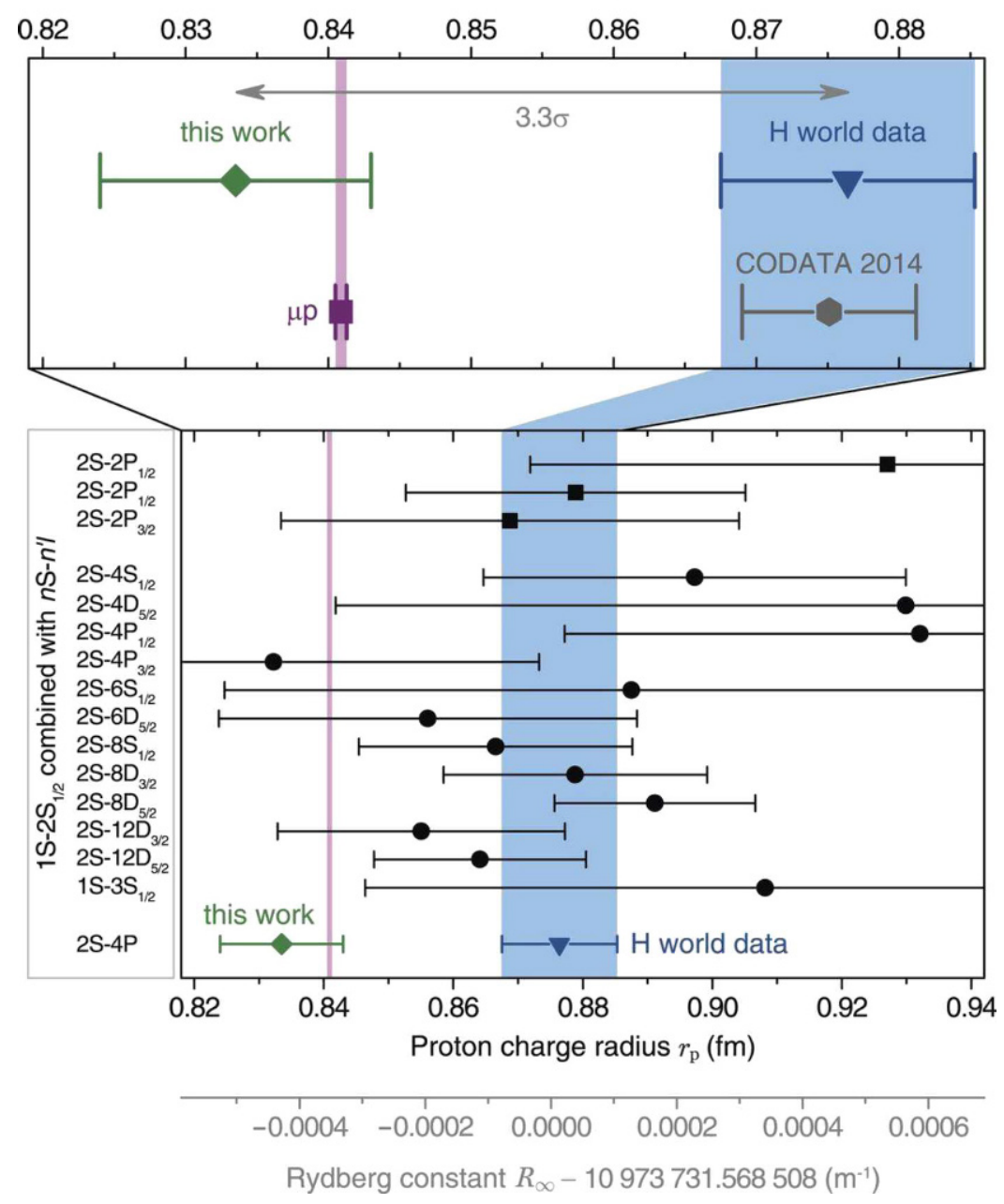

Fig. 43. Values of the r.m.s. proton charge radius $r_{p}$ derived from the recent measurement of Beyer et al. [614] (green diamond), muonic hydrogen measurements $\mu p$ (pink bar and violet square) and the value derived from world hydrogen data (blue triangle). Also shown are the individual optical and microwave measurements that together constitute the hydrogen world data value. These consist of 15 individual measurements (black circles, optical measurements; black squares, microwave measurements). The CODATA adjustment [615] includes hydrogen data as well as deuterium data (nine measurements) and electron scattering data. The plot is unchanged if given in terms of the Rydberg constant $R_{\infty}$ owing to its correlation with $r_{p}$, as indicated by the dual scale. From reference [614] reprinted with permission from AAAS.

in terms of producing the most precise measurements, data from different types of systems can provide valuable insight, even at lower levels of precision. In order to fully exploit future Ps measurements they should be conducted with an associated evaluation of the relevant theoretical corrections, although it is clear that significant experimental progress is required before additional calculations will be required.

\subsection{Scattering}

A slow beam of $\mathrm{Ps}^{*}$ atoms obtained via Stark-deceleration (see Sect. 5.1) can be spatially focused (e.g. [475]) using methods similar to the electrostatic guiding discussed in Section 3.2.2. This means that one could produce a slow focused $\mathrm{Ps}^{*}$ beam that would be ideal for studying various interactions of Ps atoms with other atoms and molecules, opening up a new realm of cold antimatter chemistry. One example of such an experiment is the formation of bound states of positrons with neutral atoms. The first suggestion that positron-atom bound states might exist came from many-body-theory calculations in 1995 [625]. Subsequent variational calculations carried out by Ryzhikh and Mitroy [626] and by Strasburger and Chojnacki [627] confirmed that positron-lithium binding can occur, and soon after, many more calculations concerning other atoms appeared [628].

There now exist many predictions of positron-atom binding for a wide variety of atoms [629-631], but there is still no experimental evidence for these states whatsoever. This is largely because one needs a mechanism to attach the positron to an atom, and also because those species that are expected to bind positrons are experimentally difficult to work with (for example, a hot cell may be 


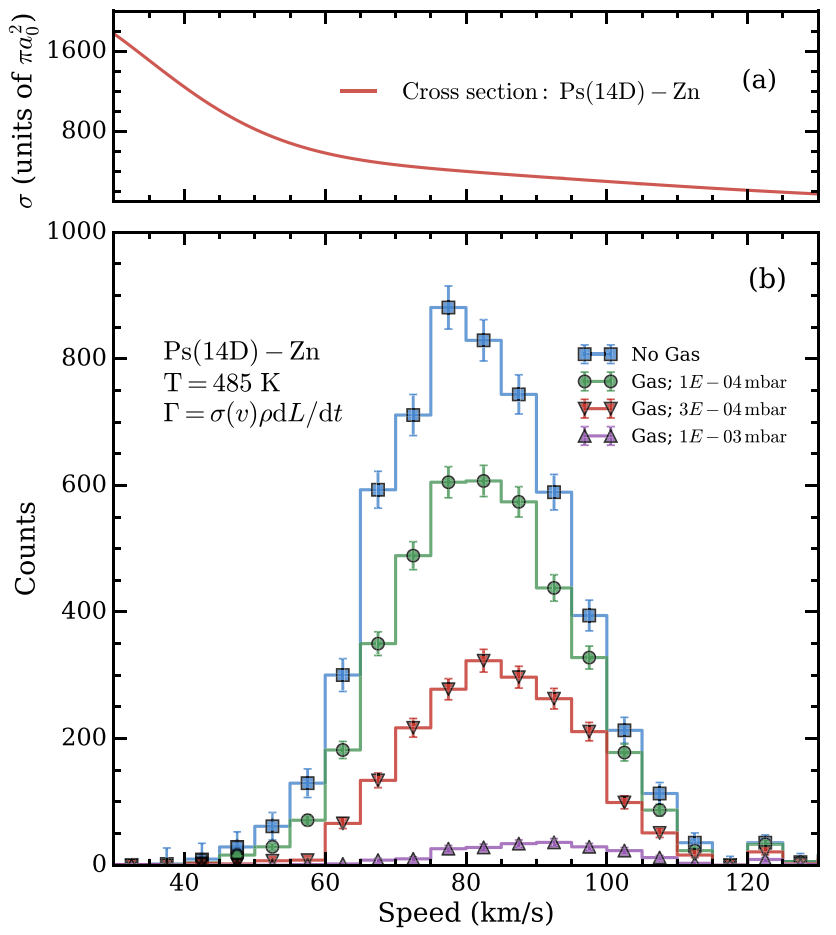

Fig. 44. (a) Calculated cross section $\sigma$ for positron-atom bound state formation following collisions of $n=14$ Ps atoms with $\mathrm{Zn}$ atoms and (b) the results of a simulation including a measured TOF Ps distribution interacting with a gas of $\mathrm{Zn}$ atoms at the pressures indicated. The scattering rate and other simulation parameters are indicated in the panel. From reference [476].

required). Some schemes for producing these systems have been suggested [632,633], including interactions with Rydberg Ps atoms [476]). This approach has the advantage that one can select the $n$ state to match the $\mathrm{e}^{+} \mathrm{A}$ binding energy, and by performing measurements as a function of $n$ obtain additional information regarding the Ps-atom interaction.

Figure 44 shows a simulation of the attenuation of Ps atoms as they pass through a gas cell of $\mathrm{Zn}$ at various pressures. The Ps TOF distribution is based on one that is measured in a quadrupole guide (see Sect. 5.1), and the cross sections for Ps-Zn scattering have been calculated [476]. These data imply that a discernible loss signal might be observed at $\mathrm{Zn}$ pressures close to $1 \times 10^{-4} \mathrm{mbar}$, although this could be lowered if slower Ps atoms were used. Such pressures can be achieved by heating Zn to temperatures $\gtrsim 500 \mathrm{~K}$ [634]. An alternative scheme would be to use a guided beam of atoms (e.g. [635]) and produce a merged beam experiment, as has been done with, for example, neutral beams of polar molecules [636,637]. In this way it would be possible to better match the projectile and target speeds, which would significantly enhance the cross sections, compensating for the reduced densities.

In addition to producing positron-atom bound systems, the electrostatic guiding methods could also be used to observe excited state Ps scattering from other atoms and molecules. Collisions between various Rydberg and neutral species have been studied for decades (e.g. [638]) and can provide information relevant to low-energy electron scattering [639]. This is because in a Rydberg atom the electron is very weakly bound, and thus in a collision it is quite possible for it to have a stronger interaction with the target species than with its own ion core. This means that these electrons can behave as if they were free electrons (with an energy given by their orbital motion).

A similar effect has already been observed in total scattering measurements between ground state Ps atoms with various atomic and molecular targets [640,641]. In some of these experiments Ps beam energies comparable to or higher than the Ps binding energy $(6.8 \mathrm{eV})$ are used, allowing the interactions to be reasonably well characterized by the impulse approximation [642,643]. For lower Ps beam energies [232] this approximation will not be valid and other effects must be taken into account [644]. It may be informative to perform similar measurements using Rydberg Ps atoms to verify the theory, and also to use atoms in different Stark-states at lower energies. This would provide a way to control the orientation of the electron and positron wavefunctions relative to the projectile axis and thus study polarization effects.

A guided beam of Rydberg Ps atoms could be used to perform charge exchange reactions with trapped charged targets. Electrostatic guides can be interfaced with standard ion traps [645] and Stark decelerators [646]. This method has been suggested as a way to produce antihydrogen formation following interactions between Ps atoms and antiprotons held in a Penning trap via the charge exchange reaction

$$
\mathrm{Ps}^{*}+\overline{\mathrm{p}} \rightarrow \overline{\mathrm{H}}+\mathrm{e}^{-} \text {. }
$$

The cross section for this reaction will be increased if excited Ps states are used $[418,419]$. Because this reaction can in principle be performed with low collision energies, and without having to merge two oppositely charged plasmas, it may be much more efficient for the creation of cold antihydrogen [647]. Experiments that have produced and trapped antihydrogen atoms have used a magnetic gradient trap that is superimposed upon a Penning trap, so that when the neutral atoms are formed (via three-body recombination), those that are cold enough will remain trapped $[648,649]$. The depth of the neutral traps used is equivalent to a temperature of less than $1 \mathrm{~K}$, which means that most of the antihydrogen produced is not trapped. Ps interactions with antiprotons could, therefore, make it possible to improve overall antihydrogen trapping rates [648].

Verifying the $n$ and velocity dependence of the antihydrogen formation cross section, as well as studying other experimental parameters that might affect antihydrogen formation rates, would be useful also to several collaborations at CERN that plan to produce antihydrogen in this way. Rather than generating trapped neutral antihydrogen these experiments are designed to produce either a beam of antihydrogen atoms in Rydberg states [650] or, following two charge exchange interactions, an antihydrogen positive ion [651]. 
One can consider other possibilities too, for example, fine control over Ps atom trajectories could also make it possible to produce a Ps-microprobe, in which atom-optics are used to direct Rydberg Ps atoms onto a target surface to be probed with high spatial resolution. The subsequent annihilation of the positron with the target material can be used to measure the local electron density as in other positron microprobes [652]. Similar methods have been used in neutral atom lithography with metastable atoms $[653,654]$, with some improvements suggested if Rydberg atoms are used [655]. As an extension, the Rydberg atoms themselves could be used as surface probes via resonant charge transfer processes [426,427]. Neutralization of laser cooled ions in Penning or Paul traps has also been suggested as a method to produce cold atoms or molecules that are not amenable to laser cooling [435].

\subsection{Bose-Einstein condensation}

Positronium is a Bosonic system and as such can be expected to form a Bose-Einstein Condensate (BEC) under the right conditions. The Ps-Ps scattering length has been calculated $[588,589]$, to be $2.95 a_{0}$ which means that BEC formation is possible: a negative scattering length implies an attractive force between atoms which tends to make BEC's unstable, although they can be produced with lower numbers of atoms [656].

The fundamental requirement for BEC formation is to achieve sufficient phase space density, which amounts to achieving a high enough particle number density and/or low enough temperature that the mean atomic separation is approximately the same as the de Broglie wavelength (see Eq. (24)). This simplistic description of a BEC overlooks numerous thermodynamic subtleties but is adequate for the present purposes: see, e.g. [657-659] for a more complete description of BEC phenomena and properties.

For a gas of Bosonic atoms with mass $m$, and density $\rho$ the BEC transition temperature $T_{\mathrm{C}}$ is given by [658]

$$
T_{\mathrm{C}}=\frac{3.31 \hbar^{2}}{2 m_{\mathrm{e}} k_{B}} \times \rho^{2 / 3} \approx 3 K \times\left(\rho\left(\mathrm{cm}^{-3}\right) / 10^{17}\right)^{2 / 3} .
$$

One can immediately see that the low mass of Ps allows for much higher critical temperatures than any other atom (although some condensed matter systems such as excitons [660] or exciton-polaritons [661] may have critical temperatures similar to or higher than Ps at equivalent densities). A Ps density of $10^{17} \mathrm{~cm}^{-3}$ would start to BoseCondense at a temperature of $\approx 3 \mathrm{~K}$. Note, however, that $T_{\mathrm{C}}$ is the temperature at which condensation starts to happen: the condensate occupancy is given by

$$
\frac{N_{0}}{N}=1-\left(\frac{T}{T_{\mathrm{C}}}\right)^{3 / 2}
$$

where $N_{0}$ is the number of atoms in the condensed phase, and $N$ is the total number of atoms in the ensemble. For $T=T_{C}$ the fraction of condensed atoms is zero, and generally the system should be considerably colder than

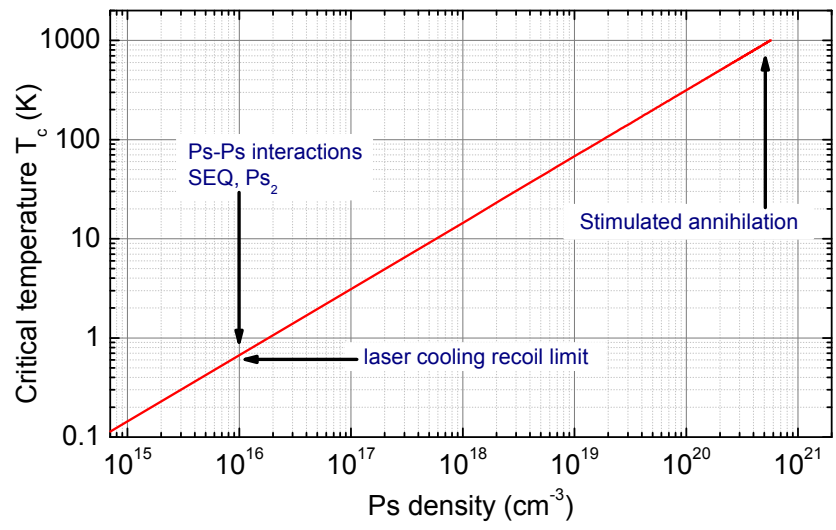

Fig. 45. Ps BEC critical temperature as a function of density as given by equation (55). Also indicated is the density at which Ps-Ps interactions have been observed, and the approximate Ps laser cooling recoil limit. The lowest density at which one might expect stimulated emission to be possible is also shown, although the actual value for this depends on the exact Ps BEC geometry and is likely to be much higher than indicated. After reference [361].

$T_{\mathrm{C}}$. Note also that the $3 / 2$ power applies to a threedimensional container, but will be different depending on how the atoms are confined. If they are in a harmonic potential well, for example, this factor will be 3 and not $3 / 2$ [171]; it is possible that it will have some other value for Ps confined in an engineered porous material if it has a strong wall interaction. The critical temperature as a function of Ps density as defined by equation (55) is shown in Figure 45.

The first BEC's to be produced in the laboratory were made from Alkali atoms [662,663], using laser cooling techniques [664-666], and the phenomena has since been observed in other systems, including hydrogen [667] and helium $[668,669]$. Platzman and Mills suggested a possible way to create a Ps BEC in 1994 [595], but this scheme has not yet been realized, although some progress has been made in this area [72,73].

It is clear that in order to create a Ps BEC one needs to increase the Ps density and reduce the Ps temperature as much as possible. In most porous structures commonly used for Ps production (see Sect. 2.2.1) Ps atoms can approach room temperature, which means that for a Ps density of the order of $10^{20} \mathrm{~cm}^{-3}$ condensation would be possible. This density is, however, four orders of magnitude higher than has been achieved so far, meaning that reducing the Ps temperature further is essential unless very significant increases in Ps density are achieved.

Ps laser cooling has been discussed for many years [73,361,384,670-672] but has not been experimentally demonstrated, even in a proof-of-principle measurement. The atomic structure of Ps does not present any fundamental limitations to Ps cooling, but annihilation of ground states is a significant limitation insofar as it limits the total amount of cooling that can be obtained using the $1^{3} \mathrm{~S}_{1}-2^{3} \mathrm{P}_{\mathrm{J}}$ transition, which is the most efficient in terms of the energy removed from the system per cooling cycle. To perform laser cooling in free space via this 
transition, the Ps atom would be in the ground state $50 \%$ of the time, and the mean annihilation lifetime would then be only twice as long as that of the ground state (i.e., $284 \mathrm{~ns}$ ). The $2^{3} \mathrm{P}_{\mathrm{J}}$ lifetime is $3.2 \mathrm{~ns}$ and since cooling necessarily requires spontaneous decay, one should wait approximately two radiative lifetimes between cooling cycles [227]. Thus, on average less than 50 cooling cycles would be possible, which would reduce the Ps temperature by $\approx 100 \mathrm{~K}[73,361]$. Cooling on other transitions that do not include the ground state have been considered [384] do not seem viable since (1) one would have to re-pump out of the ground state following spontaneous decays (with associated losses), (2) the energy removed per cycle would be much lower, and (3) the Ps ensemble would spread out, requiring very powerful lasers covering a large volume of space.

The laser sources that have been suggested for Ps cooling (e.g. [670]) are well within current technological capabilities, and laser cooling may become useful if colder ensembles of atoms can be generated, or if other cooling processes can be simultaneously employed. However, even if Ps laser cooling could be performed with optimal efficiency, the low mass of Ps limits the temperatures that can be achieved to $\approx 1 \mathrm{~K}$ because of the Ps recoil. Since Ps is very light this recoil is substantial, and in order to conserve energy and momentum there must be an associated frequency shift of the absorbed or emitted photon, which is given by $\Delta \nu_{\text {rec }}$, where

$$
\Delta \nu_{\mathrm{rec}}=\frac{h}{4 m_{\mathrm{e}} \lambda_{0}^{2}} \approx 3 \mathrm{GHz} .
$$

The recoil frequency shift is almost three orders of magnitude larger for Ps than for $\mathrm{H}$ (i.e., $\mathrm{GHz}$ vs. $\mathrm{MHz}$ ), and is even smaller for other atoms and molecules. The recoil limit for Ps is larger than the Doppler limit, which is not true for other atomic systems, where it is difficult even to observe such recoil effects [673-675].

In the context of making a Ps BEC it is necessary to draw a distinction between Ps laser cooling in free space, and in a confined volume of some sort, which is generally considered to be necessary to maintain Ps densities. As discussed in Section 2.2.1, when Ps is created in porous silica films (or other similar materials) they are initially ejected from the bulk material with $\mathrm{eV}$ energies, which is then reduced to near thermal energies on ns time scales via collisions [126]. This is equivalent to cooling from $11000 \mathrm{~K}$ to $600 \mathrm{~K}$ in less than $10 \mathrm{~ns}$, which is considerably more than can be achieved via laser cooling. This mechanism does not generally allow one to cool samples and produce cold Ps which is then emitted into vacuum because the confinement energy will be converted to kinetic energy upon emission [109]. However, while they remain in the pores, Ps atoms can cool to the ground state of the confining structure.

The situation is more complicated if one considers the structures needed for BEC production, namely a large central cavity (probably of micron dimensions) into which all Ps atoms can be collected [595]. This is a fundamentally different situation from that encountered in most existing experiments, where Ps atoms are isolated from each other and individually occupy small pores. In both cases, however, laser cooling is unlikely to be possible because Ps scattering (either from other Ps atoms or from wall interactions) will lead to Dicke line narrowing [406]. This means that atoms cannot be selected according to their velocity, and laser cooling will be ineffective. Thus, while laser cooling for a confined Ps gas can be envisioned [676], the effects of confinement would have to be explicitly addressed, either by using sufficiently large cavities or other confining structures [123], or possibly by using alternative cooling schemes employing short-pulsed lasers (e.g. $[677,678])$.

The highest Ps densities obtained to date are on the order of $10^{16} \mathrm{~cm}^{-3}$, achieved in a series of in Ps-Ps interaction experiments $[119,290,362]$. For a completely unpolarized positron source producing Ps at these densities or above leads to very significant losses since interactions between oppositely polarized Ps atoms can convert longlived atoms to short-lived states [288] with high efficiency [589] (see Sect. 3.1.3). Thus, if the primary positron beam used is partially spin polarized (as it is when derived from a radioactive source [80]), it is possible to retain a fraction of the majority Ps spin component following complete SEQ scattering [362]. However, for typical beam polarizations $(\approx 30 \%)$ this will mean losing a factor of three in the Ps density, and so employing a fully spin polarized beam might be preferential, even if the overall throughput of the system is reduced [679]. Recent developments with polarized bremsstrahlung sources may be useful in this regard as they could make it possible to generate intense pulsed and polarized positron sources with a linac [680].

Creating a dense Ps gas necessarily requires a highdensity positron pulse. In previous experiments [356] this was done by stacking positrons in an accumulator and using plasma compression methods (i.e., strong drive rotating wall techniques [353-355,593]) to increase the beam density. This beam was then further compressed with a pulsed magnetic field. However, such pulsed fields introduce numerous experimental complications, and the fields that can be generated are not easily scalable. A more fruitful approach may be to extract the positron plasma into a field-free region $[92,329,681]$ and increase the areal density by remoderation [682]. In this way one could expect to improve the beam brightness, losing approximately a factor of 10 in the number of positrons, but gaining a factor of 100 in the density [683].

It is possible to store $\approx 10^{9}$ positrons or so in a standard accumulator [684,685], and one can also consider building multiple positron traps, either in line, or using an off-axis multi-cell design $[686,687]$. In any case, high capacity traps will clearly take longer to fill unless they can be associated with an intense positron source. One possibility might be to couple the low-brightness, high-intensity NEPOMUC beam [688] to a Surko trap specifically designed to have a large diameter entry electrode. This would require much more differential pumping than is usually the case, but could result in very efficient positron capture and brightness enhancement. However, beams produced via pair production, as in reactors or linacs, are not usually spin polarized, so this approach would require additional steps 
to polarize the positrons, either via modifications to the production mechanism [680], or by manipulating unpolarized positron plasmas [689]. Multiple or high capacity traps could provide a significant Ps density enhancement, perhaps offering a factor of ten over current arrangements. Together with remoderation it may then be possible to bring Ps densities into the $10^{18}-10^{19} \mathrm{~cm}^{-3}$ range.

The technology required to generate intense positron beams with much higher densities than have been previously achieved already exists, and it is only a matter of combining some different techniques in the right way. This situation is similar to the production of high-intensity pulsed beams for laser spectroscopy in that the underlying concepts have all been around for many years [66,345], and just needed to be combined to facilitate a new methodology enabling laser spectroscopy [357]. It is not, therefore, unreasonable to believe that the formation of a Ps BEC may be on the horizon. However, I believe that this will only be true if serious work is put into the design and fabrication of micro-structures that are able to produce, cool, and collect Ps atoms in an appropriate central cavity. Not only can this potentially provide significant density enhancements and higher cooling rates, but it is also important to produce the cold Ps ensemble in a way that does not inhibit condensation. In prior work where a spin-polarized Ps gas was generated [362], Ps atoms were individually localized in a distributed network of pores. This configuration is unlikely to be conducive to BEC formation, not least because of the strong atom-wall interactions. The type and shape of the confining material (the "container") used to produce a Ps BEC may well affect its properties [690,691]. Several theoretical studies have been carried out regarding Ps condensation dynamics under various conditions (e.g. [692-697]), but many questions concerning high densities of interacting Ps atoms remain unanswered and experimental data is scarce.

In additional to subtle geometrical effects that may impact the thermodynamic properties of a condensate, more familiar problems must also be considered, such as how the BEC formation target material will react to the implantation of an intense positron beam and laser radiation. It will be necessary to avoid producing targets made from materials in which paramagnetic centers can be generated. It is well known that paramagnetic centers can be generated by radiation (e.g. [698]), including laser light [289], and even positron irradiation when intense beams are used [699]. These centers can inhibit Ps formation [700] or can convert triplets to singlets via spin exchange [701], and are therefore not compatible with Ps BEC experiments. Moreover, there are many types of paramagnetic center, and often they become more stable at lower temperatures [131,702,703], and thus could present a particularly serious problem for experiments that require cryogenic Ps.

The Ps formation target also needs to withstand the local temperature increase following deposition of an intense positron pulse: if one implants $10^{10}$ positrons with $5 \mathrm{keV}$ into a target, the total energy deposited will be $8 \mu \mathrm{J}$. This energy will be deposited is a small volume that will ultimately depend on various other experimental parameters, in particular how the Ps is to be produced and collected into a single cavity-like structure. I would estimate the likely deposition volume to be in the range $10^{-8}$ to $10^{-10} \mathrm{~cm}^{-3}$. For a tungsten target this would result in a corresponding temperature increase of 200$20000 \mathrm{~K}$ : that is to say, most targets would probably immediately melt (locally) following implantation of such a pulse.

It has been suggested $[73,704]$ that the deposited energy may be dispersed over a larger volume via ballistic phonon transport, but the required efficiency of this process seems to be unrealistic $[705,706]$. The actual energy transport would of course depend strongly on the exact nature of the structure used and the material from which it is made, imposing yet more constraints on possible structures. Alternatively, Mills has suggested using a sacrificial layer of frozen gas (e.g., Argon) on the target surface to remove the deposited energy by vaporization [73]. This may be possible but has yet to be evaluated experimentally. The problem of target heating may necessarily require a scheme wherein the positrons are implanted into a large volume structure that is engineered to collect Ps atoms in a much smaller volume. Any serious attempt to observe a Ps BEC will have to address this problem since even if targets are not vaporized, heating up the $\mathrm{Ps}_{\mathrm{s}}$ is counter productive.

If a Ps BEC were to be produced, it may not be entirely obvious that this has happened. The method originally proposed by Platzman and Mills [595] was to magnetically quench the BEC with a transverse magnetic field to induce two-photon decays, and then use an ACAR detection scheme to observe the very narrow momentum component characteristic of a BEC. This seemingly simple scheme hides numerous difficulties: ACAR detectors generally have very low detection efficiencies (they need to be far away from the radiation source to achieve good angular resolution) and also can only detect single events per detector pair, and they require two-photon decays. Thus, a large detector array is required to optimize detection. Moreover, to convert triplet atoms to singlets to facilitate two-photon decay, either a several kG spin flipping magnetic field must and applied in a time comparable to the $142 \mathrm{~ns}$ Ps lifetime, or a high-power microwave field must be applied [323,594]; neither of these are trivial endeavors [73]. Optical methods may be able to reveal the presence of a BEC and avoid these problems [73,594], although it is by no means obvious that one can unambiguously distinguish between a cold dense Ps ensemble and a Ps BEC when both are present in a material structure.

The fact that the low mass of Ps may allow near room temperature BEC formation, is not itself sufficient justification for performing such complex experiments (especially if similar properties can be studied more easily in condensed matter systems [661]). The reasons why it is worthwhile producing a Ps BEC are related to the fact that it is composed partly of antimatter, and that having access to Ps in the Bose condensed state makes some experimentation relevent to antimatter physics possible. Specifically, it may, at least in principle, enable the generation of a coherent Ps atom laser, and the observation of the phenomenon of stimulated annihilation. A Ps "atom laser" is a coherent beam of Ps atoms generated from a Ps BEC. 
If such a condensate were created in a cavity that was accessible to the vacuum, then atoms could spontaneously tunnel out, and in the process stimulate further (coherent) tunneling of other atoms into the beam (according to BE statistics) [73]. This would constitute an ideal source for precision optical spectroscopy, with negligible Doppler effects (see Sect. 5.2), and may also be amenable to interferometric experiments (see Sect. 5.5). It is not difficult to think of many other experiments that would be made possible or be greatly improved by the availability of a Ps BEC.

Stimulated annihilation is a process analogous to the more familiar stimulated emission mechanism that underlies the operation of the laser, but at a very different energy scale. For this reason the concept of stimulated annihilation is often referred to as the gamma-ray laser. Although one relies on the other, there must be some gain in order for stimulated annihilation to result in lasing. Stimulated annihilation of singlet Ps would occur if $511 \mathrm{keV}$ annihilation radiation interacted with slow atoms in such a way as to drive them to annihilate, emitting two photons, where at least one of them is coherent with the incident photon [707]. If the initial photon comes from Ps decay, then the atom must be cold enough that the annihilation radiation is not Doppler shifted out of resonance. The linewidth of this process would be on the order of $1 \mathrm{GHz}$, owing to the singlet Ps lifetime. For an atomic transition this might be considered rather broad, as it would represent on the order of one ppm of the total transition interval. For electronpositron annihilation, however, it is closer to $0.1 \mathrm{ppt}$ (the frequency scale of electron-positron annihilation is $\left.2 m_{e} c^{2} / h \approx 10^{21} \mathrm{~Hz}[708]\right)$. This means that Ps atoms would have to be extremely cold to absorb or emit radiation without being Doppler shifted out of resonance. As a result, stimulated annihilation is only feasible (to the extent that it is feasible at all) if the Ps atoms are Bosecondensed [384,707,709-712], since they will then possess a zero-momentum component [658].

The methodology for Ps BEC production proposed by Liang and Dermer [384] and also by Platzman and Mills [595] included spin flipping some fraction of the BEC into singlet states to produce two-photon decays to facilitate detection. In order to achieve stimulated annihilation it would be necessary to efficiently convert a large fraction of the condensate into singlets to generate resonant annihilation radiation. Since this annihilation radiation would be emitted isotropically the density and geometry would have to be arranged so that the probability of emission along the direction of the BEC was significant [594]. These requirements would, in practice, likely increase the required density from $\approx 10^{21} \mathrm{~cm}^{-3}$ or so suggested by the Dirac cross section by many orders of magnitude. This probably means that stimulated annihilation is not likely to be observed using existing technology. Producing a gamma ray laser (with gain $\geq 1$ ) in this way would require even higher densities. Mills has estimated various numbers in "realistic" experimental conditions [594]. I note that other schemes designed to generate tunable gamma radiation sources (both coherent and non-coherent) have been discussed (e.g. [713-718]) but none of them have yet been experimentally demonstrated.

\subsection{Antimatter gravity experiments}

The idea of "antigravity" is considered by some to be a crazy (or just wrong) idea, that is in fact already either ruled out or strictly limited by experimental data $[719,720]$, while others believe it emerges naturally from some established theories [721-723], and may be the solution to many of the biggest problems in physics [724]. For convenience I will here refer to any asymmetry between the gravitational interactions of matter and antimatter as antigravity. This could be a fully repulsive matterantimatter interaction, or just a small difference in how antimatter interacts with gravity fields generated by matter (or vice versa). Even the most optimistic antimatter gravity proponents do not appear to think that antimatter test masses in fields generated by antimatter would behave differently to matter on Earth.

If one postulates that there is a complete gravitational repulsion of matter and antimatter one can develop models [725] that lead to an oscillating Universe in which a "big crunch" generates a super-massive black hole that drives antimatter creation in a way that looks rather like a big bang. This mechanism would lead to alternating Universes, successively dominated by matter and antimatter after each expansion and contraction cycle [726], and do not require any other asymmetries or $\mathrm{CP}$ violating effects (i.e., no Sakharov criteria are required [167]) to explain the observed matter-antimatter asymmetry. Furthermore, this model can eliminate the need for an inflationary period. The existence of such a repulsive force can, if interpreted in a particular way, explain $\mathrm{CP}$ violation, galactic rotation curves, the acceleration of the expanding Universe and other observations, rendering dark matter and energy redundant [727-732]. Any consistent theory that could explain $\mathrm{CP}$ violation, dark matter, dark energy, inflation, and the matter-antimatter asymmetry all at once would of course be very appealing.

On the other hand, one also has to simultaneously explain many other things, such as: (1) Photons, which are their own antiparticles, obey the known laws of gravitation to a high degree, as demonstrated by light deflection and gravitational lensing [733,734]. (2) Since most (98\%) of the mass of nucleons comes from their binding energy (i.e., particle-antiparticle pairs), weak equivalence principle (WEP) measurements (e.g. [735]) should already exclude antigravity, at least to the extent that they confirm the WEP [736]. (3) If antimatter and matter have opposing gravitational interactions, a particle-antiparticle pair could move in a gravitational potential without doing any work, but this pair can be converted to photons, which we know are affected by gravitational fields [737]. This contradiction could in principle allow one to construct a perpetual motion machine, since it implies non-conservation of energy. (4) Simultaneous measurements of proton (actually, an $\mathrm{H}^{-}$ion) and antiproton cyclotron frequencies show no difference at the $0.1 \mathrm{ppb}$ level [738]. If CPT is assumed to be an exact symmetry 
then these measurements can be interpreted as WEP tests (at the $10^{-6}$ level), since the gravitational component of the mass-energy (and hence cyclotron frequency) of these particles would be different if antigravity exists [739]. (5) What about those neutral Kaons [740]?

As a simple experimentalist with only a limited understanding of complicated theoretical matters I am inclined to remain agnostic concerning the possibility of any matter-antimatter gravitational asymmetries. In my view there is no reason not to test such possibilities experimentally; if measurements can unambiguously confirm that antimatter is gravitationally identical to matter then this is still a significant result. Such a measurement would indicate that we shall have to look elsewhere for solutions to various unsolved mysteries, and would therefore prevent fruitless searching. However, the same logic can also be applied to testing magic healing crystals, or checking rainbows for leprechaun gold. With limited resources one has to be somewhat selective in what sorts of scientific projects are attempted, and in the absence of an imperative, directly searching for antigravity effects may seem rather profligate.

But we do have an imperative, and an urgent one at that: The Standard Model is extremely successful, and definitely incomplete. The greatest triumphs of modern physics (quantum theory and general relativity) are fundamentally incompatible; a quantum theory of gravity is desperately needed, and would presumably naturally answer all antigravity questions, but it has been impossible to create one. Whether such a theory would be generated from some sort of extension to the Standard Model [741-744] or from something else entirely (string theory?) is not known, which is why there is so much room for speculation. Thus, arguments [722,745] and counter arguments $[746,747]$ in both directions can be generated indefinitely. Indeed, one could write an entire paper just about these rationalizations (cf. [170]), but ultimately data will have the last word.

The most direct experiment one can envision to check for antigravity would be to simply drop some antimatter and see if it falls at the same rate as matter. Unfortunately this is not as easy as it sounds. The stable antimatter particles we can most easily obtain are positrons and antiprotons, and in principle we could devise an experiment to drop these (that is to say, perform a free-fall measurement [748]). The problem is that measuring gravitational forces on charged particles is extremely difficult [749], not only because gravitational effects can be overwhelmed by very small $\left(\approx 10^{-12} \mathrm{Vm}^{-1}\right)$ electric fields, but also because the electric fields present in conductors are themselves affected by gravity, but in a way that is not necessarily understood [750-752]. For this reason only experiments with neutral systems that are completely or partially composed of antimatter particles are currently being pursued, namely, antihydrogen, muonium and positronium. These systems all have their own specific advantages and challenges.

Low-energy antiprotons are only presently available at CERN, which means that antihydrogen experimentation in this area is limited to a few mid-sized (by CERN standards) collaborations. Since antihydrogen is intrinsically stable (i.e., it does not self-annihilate) it is the best system available for antigravity studies. Antihydrogen [753] was produced at CERN two decades ago in energetic beams $[754,755]$. However, producing cold antihydrogen atoms is necessary for any useful measurements, which is significantly more difficult. Nevertheless, it has been accomplished using trapped positrons and antiprotons by the ALPHA, ATRAP and ASACUSA collaborations $[87,756,757]$. Capturing these antihydrogen atoms in magnetic gradient (Ioffe) neutral atom traps is also very challenging, but this too has been done $[89,649]$.

The antihydrogen experiments conducted so far are primarily aimed at performing CPT tests via spectroscopic measurements [758], although one preliminary gravity test (setting extremely broad limits on possible antihydrogen antigravity) has been conducted [759]. Several newer collaborations have been established specifically to measure gravitational interactions involving antihydrogen $[651,760]$. These groups intend to generate antihydrogn via interactions of trapped antiprotons with excited state Ps atoms, which increases the antihydrogen formation cross section substantially $[418,419,432,647]$. This method has been demonstrated experimentally by the ATRAP collaboration using Ps atoms generated via charge exchange with Rydberg Cs atoms [416,761]. The new antihydrogen gravity experiments intend to use direct laser excitation to generate excited state Ps atoms [135,762], and thus may benefit from techniques similar to those described in this article.

Positronium has also been suggested as a possible subject of gravitational studies $[477,763]$. Being unstable it is less attractive than antihydrogen in this regard, but this is offset by the fact that it is very much easier to produce. No Ps gravity experiments have been completed yet, but various aspects of doing so have been discussed in the literature $[411,477,599,764,765]$. Many of the recent developments in Ps excitation and manipulation discussed in this review (e.g. $[136,141])$ are of direct relevance to possible Ps gravity experiments. A simple free-fall experiment, for example, can only succeed if Ps atoms are excited to long-lived Rydberg states. Moreover, one would also need to generate a Ps beam that was narrow, slow, and focused well enough to allow deflections on the order of 10's of microns or more. This requires lifetimes on the ms scale, which is challenging (see Sect. 3.2). At typical Ps speeds a $1 \mathrm{~ms}$ flight time implies a $100 \mathrm{~m}$ long vacuum chamber, which is clearly impractical. For such long lifetimes black body radiation may well have to be reduced using a cooled drift tube, which adds to the impracticality of a long flight path. Clearly, colder atoms or the application of Stark deceleration methods [338] will be required to perform Ps free-fall experiments.

Although the large dipole moments of some Rydberg states allows beams to be controlled using external inhomogeneous electric fields, this benefit becomes a disadvantage for gravity experiments, since weak stray field gradients that are typical of real experimental conditions can affect atom trajectories as much as gravitational deflections: this rather defeats the point of using electrically neutral systems for gravity measurements. It is possible to excite Ps to Rydberg-stark states that do 
not have any dipole moments, at least to first order (i.e., to $k=0$ states). In fact, the long lifetimes required can more easily be achieved using circular states (states with maximal angular momentum [175]), which do not have dipole moments. This means that any experiment that relies on electric fields to guide, focus, or decelerate Ps atoms to generate a beam may have to also include another step in which the prepared atoms are transferred to the required circular states via microwave transitions (the crossed-field method for producing circular states [766] cannot be used with Ps). New experiments are ongoing to discover efficient methods to do this.

Muonium, the hydrogenic bound state of an electron and an antimuon, was first produced by Hughes and co-workers in the 1960s [767], and has been studied via laser spectroscopy in several experiments [768,769]. The antimuon is intrinsically unstable and decays into a positron, an electron neutrino, and a muon antineutrino, with a lifetime of $2.2 \mu \mathrm{s}$ [770]. They are created using high energy proton beams and so muonium production is tied to a handful of accelerator facilities, with the Paul Scherrer Institute (PSI) in Zürich currently the most active in muonium research (e.g. [771,772]). Gravity measurements using muonium have been proposed [773], but since the muonium lifetime is essentially the same as that of the muon, this is very challenging. Being both unstable (like positronium) and hard to produce (like antihydrogen), muonium is perhaps the least attractive system for such tests.

Free-fall measurements are intrinsically limited by the fact that it takes a long time to fall a small distance: exciting Ps atoms to states that live for ms yields only micron deflections, and scaling up any such experiment to make more precise measurements of $g$ is not feasible. One could instead consider performing interferometry experiments which are much more sensitive and in general more amenable to improvements. The sensitivity might even allow for measurements to be performed using charged particle interferometers [774] of various types (e.g. a Ramsey Bordé interferometer [775] or schemes based upon spin-polarization $[776,777])$. However, these may only be viable for gravity tests if one could devise a method in which phase shifts developed from gravitational effects could somehow be differentiated from other sources, e.g. by rotating the system with respect to the gravitational field. Alternatively, atom interferometry $[778,779]$ is a well-developed field that is routinely used to perform high precision measurements of the gravitational field of the Earth (e.g. [780-786]). Interferometry of antihydrogen using a Ramsey-Bordé approach [787] has been proposed [788].

Various interferometry schemes are possible [779], each with pros and cons when applied to Ps. A distinct advantage in some cases (e.g. [789]) is that the low Ps mass leads to a correspondingly long de Broglie wavelength $\lambda_{\mathrm{dB}}$; for room temperature Ps atoms $\lambda_{\mathrm{dB}}$ is close to $4 \mathrm{nn}$ (see Eq. (24)), whereas room temperature. He has a de Broglie wavelength of just under $0.1 \mathrm{~nm}$. One problem is that, in the ground state, Ps lifetimes are too short for some configurations, either because the corresponding physical size of the interferometer is not realizable, or that there is not enough time to accrue a sufficiently large phase shift. Ground state Ps atoms with speeds $\gg 1 \mathrm{eV}[790]$ can be detected directly with an MCP, and hence could be observed with a spatial resolution on the 10 micron scale, but these are probably too fast to be useful. Fluorescence based methods are not possible due to very low numbers of atoms. These problems can be avoided by exciting Ps atoms to long-lived Rydberg levels, which can be detected on an MCP at low energies owing to their $\geq 5 \mathrm{eV}$ internal energy [138]. However, using Rydberg atoms may introduce new obstacles that must be overcome.

There is no experimental demonstration of diffraction or interference using Rydberg atoms, although they have been observed with metastable rare gas atoms [791-793]. This may simply be because nobody has seen any reason to perform such an experiment. However, since Rydberg atoms are in general very sensitive to external fields and can easily be ionized $[160,423,424]$, they may not be compatible with transmission through physical gratings. Possibly experiments have been tried that just did not work. It is known that the transmission of Rydberg atoms through metal slits with micron scale separations can be affected by atom-surface interactions [794,795]. Gratings constructed from insulating materials can be used for neutral atoms, but even small amounts of charging in the grating structure could result in large electric fields, which are not compatible with Rydberg atoms. Conversely, using light to generate gratings [796,797] may be compatible with Rydberg atoms, and would have the advantage that different grating parameters could be more easily obtained.

Ps atom interferometry has not been achieved experimentally, although some discussion exists in the literature $[764,765,798]$. The way in which Ps atoms are produced means that it is not possible to generate coherent atom sources. However, experiments have been performed in which diffraction and interference effects have been observed using non-coherent sources of large molecules [799]. The molecular beams required for these experiments are not very different in intensity or brightness from Ps beams, and some of the techniques developed for this work may be applicable to Ps experiments. Oberthaler has discussed using near-resonant standing light waves to perform Bragg diffraction measurements with positronium atoms [765]. A scheme is described which employs a coherent splitting mechanism with light waves acting as gratings in a Mach-Zehnder configuration. This would allow, in principle, a Positronium interferometer capable of measuring gravitational acceleration to be produced. Recoil effects and large Ps velocity distributions, as well as low numbers of atoms and limited detection methods are problems that may be addressed using a variety of recently developed interferometry techniques (e.g. [800,801]).

\section{Concluding remarks}

As is evident from Table 1, experiments involving laser excitation of Ps are becoming more widespread, and are starting to expand into new areas. Of course, many of 
these areas are only new for Ps, and have been studied at length using other atomic and molecular systems, but this just means that there are many well established techniques that can be applied to Ps experiments. The problems related to weak positron sources, fast and divergent Ps atoms, and short Ps lifetimes are still limiting factors in many experiments, but they can be mitigated by using Surko traps, and by exciting atoms to longlived Rydberg states. There can be no question that these and other advances will lead to significant new positronium experiments being performed in the coming years. I have mentioned a few of them in Section 5, but these are just the obvious ones. Ps deceleration and trapping are imminent, and will surely inform other areas. In particular, one promising new area that will benefit from this work are studies of low-energy Ps* interactions with other atoms and molecules; these experiments could open up new analytic techniques for molecular spectroscopy, much as positron-molecule experiments have [86].

In my opinion the single most useful advance in Ps physics today would be the development of an efficient source of cold Ps atoms. In this context the definition of "cold" really depends on specific experiments, but a source of atoms with mean energies of $\leq 10 \mathrm{meV}$, with a spread of $\leq 1 \mathrm{meV}$ would be significantly different from anything currently available. In this range Stark manipulation techniques would be much more effective, and could likely be performed with maximal efficiency. This would be beneficial to just about any experiment one wishes to conduct with Ps in excited states, whether it is due to more efficient excitation with narrower lasers, better spectral resolution, or a larger acceptance for Stark-manipulation devices such as lenses, guides, decelerators, or traps.

It seems unlikely to me (but not to everyone [73]) that laser cooling of Ps will ever be a worthwhile means of producing atoms for use in other experiments. Even if it were performed in the most optimal way possible, the overall efficiency is intrinsically limited by the ground state lifetime, and without trapping them Ps atoms will be moving away from their source by many $\mathrm{cm}$ in this time. It may be that producing a cold distribution (e.g., by oxygen assisted thermal desorption from a metal surface [235]) and then velocity-selecting the coldest part will always be competitive will laser cooling; certainly this is considerably easier to implement. Possibly transitions that do not involve the ground state can be used, but only if the atoms are not spreading out to much, which would essentially mean they were already cold. Alternatively, it may be possible to contain Ps atoms in some sort of physical structure that does not inhibit laser interactions [123] and achieve some cooling. Although I am skeptical of the utility of such a scheme, if it does turn out to be possible it could allow fine control of the Ps temperature in a BEC experiment and provide a way to scan through $T_{\mathrm{C}}$. This approach may also be useful for laser cooling Muonium [772].

The collisional cooling of Ps that occurs in mesoporous silica films is already extremely effective: Ps atoms are emitted into the voids with energies of around $1 \mathrm{eV}$ [239], which corresponds to a temperature of more than $10000 \mathrm{~K}$, and they essentially thermalize to room temperature in a few 10's of ns [802]. To accomplish such a cooling rate with a laser would be an astounding achievement, and it seems reasonable to conclude that the most significant progress in obtaining cold Ps sources will be made by advances in engineered structures in mesoporous materials. However, it is true that the cooling rate decreases as cooling proceeds, so that at some point an additional cooling mechanism may be required. This could be laser cooling, or it could be modified collisional cooling, which can be achieved by introducing low mass molecules that increase the energy loss per collision [280]. Other avenues may also be possible: for example, the availability of narrow velocity distributions hinted at by MOF measurements [124] is a promising area for future research, especially considering the huge number of possible MOF materials. The production of macroscopic single crystals may be an impediment to this work, but further studies are certainly warranted.

In any case, the increased availability of cold Ps and new designs for Ps formation materials will surely enable much new physics. It does not seem overly optimistic to expect advanced Stark-based Ps trapping and manipulation techniques to be developed in the next few years, and for this to enable new precision spectroscopy measurements. Ps scattering experiments using slow Rydberg atoms are already taking place, as are the early steps leading towards a free-fall measurement. The possibility of performing both diffraction and interferometry with Rydberg Ps is being investigated, and may well inform a new approach to Ps gravity measurements. Ps BEC formation is the most challenging of the next generation experiments, but in terms of the progress already made, the few orders of magnitude increase in phase space density required does not seem entirely intractable.

I am grateful to many people for useful discussions, reading parts of the manuscript, and/or providing figures, namely: Mike Charlton, Paolo Crivelli, Gleb Gribakin, Stephen Hogan, Adric Jones, Laszlo Liskay, Koji Michishio, Allen Mills, Yasuyuki Nagashima, Randolf Pohl, and Cliff Surko. Experimental work at UCL was done mostly by post doctoral research assistants A. Deller and T.E. Wall, and graduate students A.M. Alonso, B.S. Cooper and L. Gurung.

\section{Author contribution statement}

This review article contains descriptions of work carried out by myself and others, but I am its sole author and take full responsibility for any errors or misstatements. I have offered what is only my opinion, which is subject to continuous revision.

Open Access This is an open access article distributed under the terms of the Creative Commons Attribution License (http://creativecommons.org/licenses/by/4.0), which permits unrestricted use, distribution, and reproduction in any medium, provided the original work is properly cited. 


\section{References}

1. A. Schuster, Nature 58, 367 (1898)

2. P.A.M. Dirac, Proc. R. Soc. Lond. A: Math. Phys. Eng. Sci. 117, 610 (1928)

3. P.A.M. Dirac, Proc. R. Soc. Lond. A: Math. Phys. Eng. Sci. 126, 360 (1930)

4. P.A.M. Dirac, Proc. R. Soc. Lond. A: Math. Phys. Eng. Sci. 133, 60 (1931)

5. P.A.M. Dirac, The principles of quantum mechanics (Clarendon Press, Oxford, 1930)

6. C.D. Anderson, Science 76, 238 (1932)

7. C.D. Anderson, Phys. Rev. 43, 491 (1933)

8. P.M.S. Blackett, G.P.S. Occhialini, Proc. R. Soc. Lond. A: Math. Phys. Eng. Sci. 139, 699 (1933)

9. S. Mohorovičić, Astron. Nachr. 253, 93 (1934)

10. H. Kragh, J. Chem. Educ. 67, 196 (1990)

11. M. Randić, Croat. Chem. Acta 82, 791 (2009)

12. J. Pirenne, Ph.D. thesis, University of Paris (1944)

13. J. Pirenne, Arch. Sci. Phys. Nat. 28, 233 (1946)

14. G. Beck, Phys. Rev. 69, 532 (1946)

15. A.E. Ruark, Phys. Rev. 68, 278 (1945)

16. J.A. Wheeler, Ann. N. Y. Acad. Sci. 48, 219 (1946)

17. M. Deutsch, Phys. Rev. 82, 455 (1951)

18. T.A. Pond, Phys. Rev. 85, 489 (1952)

19. M. Deutsch, Phys. Rev. 83, 866 (1951)

20. A. Ore, J.L. Powell, Phys. Rev. 75, 1696 (1949)

21. M. Deutsch, E. Dulit, Phys. Rev. 84, 601 (1951)

22. T.A. Pond, R.H. Dicke, Phys. Rev. 85, 489 (1952)

23. M. Deutsch, S.C. Brown, Phys. Rev. 85, 1047 (1952)

24. M. Weinstein, R. Deutsch, S.C. Brown, Phys. Rev. 94, 758 (1954)

25. M. Weinstein, R. Deutsch, S.C. Brown, Phys. Rev. 98, 223 (1955)

26. H.W. Kendall, M. Deutsch, Phys. Rev. 101, 20 (1956)

27. M.H. Weber, A.W. Hunt, J.A. Golovchenko, K.G. Lynn, Phys. Rev. Lett. 83, 4658 (1999)

28. A.W. Hunt, D.B. Cassidy, P.A. Sterne, T.E. Cowan, R.H. Howell, K.G. Lynn, J.A. Golovchenko, Phys. Rev. Lett. 86, $5612(2001)$

29. J. Wheatley, D. Halliday, Phys. Rev. 88, 424 (1952)

30. V.W. Hughes, S. Marder, C.S. Wu, Phys. Rev. 98, 1840 (1955)

31. V.L. Telegdi, J.C. Sens, D.D. Yovanovitch, S.D. Warshaw, Phys. Rev. 104, 867 (1956)

32. S. Marder, V.W. Hughes, C.S. Wu, W. Bennett, Phys. Rev. 103, 1258 (1956)

33. R.L. Brock, J.F. Streib, Phys. Rev. 109, 399 (1958)

34. W. Brandt, S. Berko, W.W. Walker, Phys. Rev. 120, 1289 (1960)

35. W. Brandt, R. Paulin, Phys. Rev. Lett. 21, 193 (1968)

36. R. Paulin, G. Ambrosino, J. Phys. France 29, 263 (1968)

37. C.V. Briscoe, S.I. Choi, A.T. Stewart, Phys. Rev. Lett. 20, 493 (1968)

38. L. Dick, L. Feuvrais, L. Madansky, V. Telegdi, Phys. Lett. 3, 326 (1963)

39. A.P. Mills Jr., S. Berko, Phys. Rev. Lett. 18, 420 (1967)

40. E.D. Theriot, R.H. Beers, V.W. Hughes, Phys. Rev. Lett. 18, 767 (1967)

41. T.C. Griffith, G.R. Heyland, Nature 269, 109 (1977)

42. L. Madansky, F. Rasetti, Phys. Rev. 79, 397 (1950)

43. D.G. Costello, D.E. Groce, D.F. Herring, J.W. McGowan, Phys. Rev. B 5, 1433 (1972)
44. K.F. Canter, P.G. Coleman, T.C. Griffith, G.R. Heyland, J. Phys. B 5, L167 (1972)

45. B. Jaduszliwer, W.C. Keever, D.A.L. Paul, Can. J. Phys. 50, 1414 (1972)

46. P.G. Coleman, T.C. Griffith, G.R. Heyland, Proc. R. Soc. Lond. A: Math. Phys. Eng. Sci. 331, 561 (1973)

47. K.F. Canter, A.P. Mills Jr., S. Berko, Phys. Rev. Lett. 33, 7 (1974)

48. A.P. Mills Jr., P.M. Platzman, B.L. Brown, Phys. Rev. Lett. 41, 1076 (1978)

49. S. Pendyala, P. Zitzewitz, J. McGowan, P. Orth, Phys. Lett. A 43, 298 (1973)

50. A.P. Mills Jr., Appl. Phys. Lett. 35, 427 (1979)

51. A.P. Mills Jr., Appl. Phys. Lett. 37, 667 (1980)

52. A.P. Mills Jr., L. Pfeiffer, Phys. Rev. Lett. 43, 1961 (1979)

53. A.P. Mills Jr., E.M. Gullikson, Appl. Phys. Lett. 49, 1121 (1986)

54. R. Khatri, M. Charlton, P. Sferlazzo, K.G. Lynn, A.P. Mills Jr., L.O. Roellig, Appl. Phys. Lett. 57, 2374 (1990)

55. G.R. Massoumi, N. Hozhabri, W.N. Lennard, P.J. Schultz, S.F. Baert, H.H. Jorch, A.H. Weiss, Rev. Sci. Instrum. 62, 1460 (1991)

56. A.P. Mills Jr., S.S. Voris, T.S. Andrew, J. Appl. Phys. 76, 2556 (1994)

57. D. Vasumathi, G. Amarendra, K.F. Canter, A.P. Mills Jr., Appl. Surf. Sci. 85, 154 (1995)

58. M. Petkov, K. Lynn, L. Roellig, T. Troev, Appl. Surf. Sci. 116, 13 (1997)

59. I.N. Meshkov, V.N. Pavlov, A.O. Sidorin, S.L. Yakovenko, Instrum. Exp. Tech. 50, 639 (2007)

60. C.D. Molek, C. Michael Lindsay, M.E. Fajardo, Rev. Sci. Instrum, 84, 035106 (2013)

61. M. Maekawa, Y. Fukaya, A. Yabuuchi, I. Mochizuki, A. Kawasuso, Nucl. Instrum. Methods Phys. Res. B: Beam Interact. Mater. At. 308, 9 (2013)

62. T. Griffith, in Advances in atomic and molecular physics, edited by D. Bates, B. Bederson (Academic Press, 1986), Vol. 22, pp. $37-75$

63. A. Dupasquier, A. Zecca, La Rivista del Nuovo Cimento (1978-1999) 8, 1 (1985)

64. M. Charlton, Rep. Prog. Phys. 48, 737 (1985)

65. P.J. Schultz, K.G. Lynn, Rev. Mod. Phys. 60, 701 (1988)

66. C.M. Surko, M. Leventhal, A. Passner, Phys. Rev. Lett. 62, 901 (1989)

67. C.M. Surko, T.J. Murphy, Phys. Rev. A 46, 5696 (1992)

68. C.M. Surko, R.G. Greaves, Phys. Plasmas 11, 2333 (2004)

69. J.R. Danielson, D.H.E. Dubin, R.G. Greaves, C.M. Surko, Rev. Mod. Phys. 87, 247 (2015)

70. A. Rich, Rev. Mod. Phys. 53, 127 (1981)

71. S. Berko, H.N. Pendleton, Annu. Rev. Nucl. Part. Sci. 30, 543 (1980)

72. D.B. Cassidy, Physics with many positrons, in Proceedings of the International School of Physics 'Enrico Fermi' Course CLXXIV "Physics with Many Positrons", edited by R.S. Brusa, A. Dupasquier, A.P. Mills Jr. (IOS Press, Amsterdam, 2010), pp. 1-75

73. A.P. Mills Jr. Physics with many positrons, in Proceedings of the International School of Physics 'Enrico Fermi' Course CLXXIV "Physics with Many Positrons", edited by R.S. Brusa, A. Dupasquier, A.P. Mills Jr. (IOS Press, Amsterdam, 2010), pp. 77-187 
74. A.P. Mills Jr., J. Phys. Conf. Ser. 505, 012039 (2014)

75. A.P. Mills Jr., J. Phys. Conf. Ser. 488, 012001 (2014)

76. A.P. Mills Jr., in Advances in atomic, molecular, and optical physics, edited by C.C.L. Ennio Arimondo, S.F. Yelin (Academic Press, 2016), Vol. 65, pp. 265-290

77. Y. Nagashima, Phys. Rep. 545, 95 (2014)

78. M. Charlton, J.W. Humberston, in Positron physics, Cambridge monographs on atomic, molecular and chemical physics, 1st edn. (Cambridge University Press, Cambridge, 2001), Vol. II

79. R. Krause-Rehberg, H. Leipner, Positron annihilation in semiconductors: defect studies, Springer series in solidstate sciences (Springer, Berlin, Heidelberg, 2010)

80. P.G. Coleman, Positron beams and their applications, 1st edn. (World Scientific Publishing Co., Singapore, 2000)

81. A. Ishida, T. Namba, S. Asai, T. Kobayashi, H. Saito, M. Yoshida, K. Tanaka, A. Yamamoto, Phys. Lett. B 734, 338 (2014)

82. R.S. Vallery, P.W. Zitzewitz, D.W. Gidley, Phys. Rev. Lett. 90, 203402 (2003)

83. O. Jinnouchi, S. Asai, T. Kobayashi, Phys. Lett. B 572, $117(2003)$

84. C.M. Surko, G.F. Gribakin, S.J. Buckman, J. Phys. B: At. Mol. Opt. Phys. 38, R57 (2005)

85. G. Laricchia, S. Armitage, Á. Kövér, D. Murtagh, in Advances in atomic, molecular, and optical physics (Academic Press, 2008), Vol. 56, pp. 1-47

86. G.F. Gribakin, J.A. Young, C.M. Surko, Rev. Mod. Phys. 82, 2557 (2010)

87. M. Amoretti et al., Nature 419, 456 (2002)

88. G. Gabrielse et al. (ATRAP Collaboration), Phys. Rev. Lett. 89, 233401 (2002)

89. T.A. Collaboration, Nat. Phys. 7, 558 (2011)

90. M. Ahmadi et al., Nature 541, 506 (2016)

91. D.B. Cassidy, K.F. Canter, R.E. Shefer, R.E. Klinkowstein, B.J. Hughey, Nucl. Instrum. Methods Phys. Res. Sect. B: Beam Interact. Mater. At. 195, 442 (2002)

92. T.R. Weber, J.R. Danielson, C.M. Surko, Phys. Plasmas 17, $123507(2010)$

93. C. Hugenschmidt, C. Piochacz, M. Reiner, K. Schreckenbach, New J. Phys. 14, 055027 (2012)

94. F. Tuomisto, I. Makkonen, Rev. Mod. Phys. 85, 1583 (2013)

95. C. Hugenschmidt, Surf. Sci. Rep. 71, 547 (2016)

96. I.J. Rosenberg, A.H. Weiss, K.F. Canter, Phys. Rev. Lett. 44, 1139 (1980)

97. T.N. Horsky et al., Phys. Rev. Lett. 62, 1876 (1989)

98. A. Weiss, R. Mayer, M. Jibaly, C. Lei, D. Mehl, K.G. Lynn, Phys. Rev. Lett. 61, 2245 (1988)

99. J. Mayer, C. Hugenschmidt, K. Schreckenbach, Phys. Rev. Lett. 105, 207401 (2010)

100. H.W. Kendall, Ph.D. thesis, Massachusetts Institute of Technology (1954)

101. S. Varghese, E. Ensberg, V. Hughes, I. Lindgren, Phys. Lett. A 49, 415 (1974)

102. S. Chu, A.P. Mills Jr., Phys. Rev. Lett. 48, 1333 (1982)

103. S. Chu, A.P. Mills Jr., J.L. Hall, Phys. Rev. Lett. 52, 1689 (1984)

104. K.P. Ziock, C.D. Dermer, R.H. Howell, F. Magnotta, K.M. Jones, J. Phys. B 23, 329 (1990)

105. K.P. Ziock, R.H. Howell, F. Magnotta, R.A. Failor, K.M. Jones, Phys. Rev. Lett. 64, 2366 (1990)
106. M.S. Fee, A.P. Mills Jr., E.D. Shaw, R.J. Chichester, D.M. Zuckerman, S. Chu, K. Danzmann, Phys. Rev. A 44, R5 (1991)

107. M.S. Fee, A.P. Mills Jr., S. Chu, E.D. Shaw, K. Danzmann, R.J. Chichester, D.M. Zuckerman, Phys. Rev. Lett. 70, 1397 (1993)

108. M.S. Fee, S. Chu, A.P. Mills Jr., R.J. Chichester, D.M. Zuckerman, E.D. Shaw, K. Danzmann, Phys. Rev. A 48, 192 (1993)

109. D.B. Cassidy, P. Crivelli, T.H. Hisakado, L. Liszkay, V.E. Meligne, P. Perez, H.W.K. Tom, A.P. Mills Jr., Phys. Rev. A 81, 012715 (2010)

110. D.B. Cassidy, T.H. Hisakado, V.E. Meligne, H.W.K. Tom, A.P. Mills Jr., Phys. Rev. A 82, 052511 (2010)

111. D.B. Cassidy, T.H. Hisakado, H.W.K. Tom, A.P. Mills Jr., Phys. Rev. Lett. 106, 133401 (2011)

112. D.B. Cassidy, T.H. Hisakado, H.W.K. Tom, A.P. Mills Jr., Phys. Rev. Lett. 107, 033401 (2011)

113. D. Cassidy, T. Hisakado, H. Tom, A.P. Mills Jr., Phys. Rev. B 84, 195312 (2011)

114. D.B. Cassidy, T.H. Hisakado, H.W.K. Tom, A.P. Mills, Phys. Rev. B 86, 155303 (2012)

115. D.B. Cassidy, M.W.J. Bromley, L.C. Cota, T.H. Hisakado, H.W.K. Tom, A.P. Mills Jr., Phys. Rev. Lett. 106, 023401 (2011)

116. K. Michishio, T. Tachibana, H. Terabe, A. Igarashi, K. Wada, T. Kuga, A. Yagishita, T. Hyodo, Y. Nagashima, Phys. Rev. Lett. 106, 153401 (2011)

117. D.B. Cassidy, T.H. Hisakado, H.W.K. Tom, A.P. Mills Jr., Phys. Rev. Lett. 106, 173401 (2011)

118. D.B. Cassidy, T.H. Hisakado, H.W.K. Tom, A.P. Mills Jr., Phys. Rev. Lett. 108, 043401 (2012)

119. D.B. Cassidy, T.H. Hisakado, H.W.K. Tom, A.P. Mills Jr., Phys. Rev. Lett. 108, 133402 (2012)

120. K. Michishio, T. Tachibana, R.H. Suzuki, K. Wada, A. Yagishita, T. Hyodo, Y. Nagashima, Appl. Phys. Lett. 100, 254102 (2012)

121. D.B. Cassidy, T.H. Hisakado, H.W.K. Tom, A.P. Mills Jr., Phys. Rev. Lett. 109, 073401 (2012)

122. A.C.L. Jones, T.H. Hisakado, H.J. Goldman, H.W.K. Tom, A.P. Mills Jr., D.B. Cassidy, Phys. Rev. A 90, 012503 (2014)

123. D.A. Cooke et al., Hyperfine Interact. 233, 67 (2015)

124. A.C.L. Jones, H.J. Goldman, Q. Zhai, P. Feng, H.W.K. Tom, A.P. Mills Jr., Phys. Rev. Lett. 114, 153201 (2015)

125. T.E. Wall, A.M. Alonso, B.S. Cooper, A. Deller, S.D. Hogan, D.B. Cassidy, Phys. Rev. Lett. 114, 173001 (2015)

126. A. Deller, B.S. Cooper, T.E. Wall, D.B. Cassidy, New J. Phys. 17, 043059 (2015)

127. A. Deller, D. Edwards, T. Mortensen, C.A. Isaac, D.P. van der Werf, H.H. Telle, M. Charlton, J. Phys. B: At. Mol. Opt. Phys. 48, 175001 (2015)

128. S.L. Andersen, D.B. Cassidy, J. Chevallier, B.S. Cooper, A. Deller, T.E. Wall, U.I. Uggerhøj, J. Phys. B: At. Mol. Opt. Phys. 48, 204003 (2015)

129. A.M. Alonso, B.S. Cooper, A. Deller, S.D. Hogan, D.B. Cassidy, Phys. Rev. Lett. 115, 183401 (2015)

130. A.M. Alonso, B.S. Cooper, A. Deller, S.D. Hogan, D.B. Cassidy, Phys. Rev. A 93, 012506 (2016)

131. B.S. Cooper, A.M. Alonso, A. Deller, L. Liszkay, D.B. Cassidy, Phys. Rev. B 93, 125305 (2016) 
132. A.C.L. Jones, T.H. Hisakado, H.J. Goldman, H.W.K. Tom, A.P. Mills Jr., J. Phys. B: At. Mol. Opt. Phys. 49, 064006 (2016)

133. K. Michishio, T. Kanai, S. Kuma, T. Azuma, K. Wada, I. Mochizuki, T. Hyodo, A. Yagishita, Y. Nagashima, Nat. Commun. 7, 11060 (2016)

134. A. Deller, A.M. Alonso, B.S. Cooper, S.D. Hogan, D.B. Cassidy, Phys. Rev. A 93, 062513 (2016)

135. S. Aghion et al. (AEgIS Collaboration), Phys. Rev. A 94, 012507 (2016)

136. A. Deller, A.M. Alonso, B.S. Cooper, S.D. Hogan, D.B. Cassidy, Phys. Rev. Lett. 117, 073202 (2016)

137. A.C.L. Jones, H.J. Rutbeck-Goldman, T.H. Hisakado, A.M. Piñeiro, H.W.K. Tom, A.P. Mills Jr., B. Barbiellini, J. Kuriplach, Phys. Rev. Lett. 117, 216402 (2016)

138. A.C.L. Jones, A.M. Piñeiro, E.E. Roeder, H.J. RutbeckGoldman, H.W.K. Tom, A.P. Mills Jr., Rev. Sci. Instrum. 87, 113307 (2016)

139. A.M. Alonso, S.D. Hogan, D.B. Cassidy, Phys. Rev. A 95, $033408(2017)$

140. A.M. Alonso, B.S. Cooper, A. Deller, L. Gurung, S.D. Hogan, D.B. Cassidy, Phys. Rev. A 95, 053409 (2017)

141. A.C.L. Jones et al., Phys. Rev. Lett. 119, 053201 (2017)

142. W. Bernreuther, O. Nachtmann, Z. Phys. C: Part. Fields 11, 235 (1981)

143. A. Pokraka, A. Czarnecki, Phys. Rev. D 96, 093002 (2017)

144. S.G. Karshenboim, Int. J. Mod. Phys. A 19, 3879 (2004)

145. S.G. Karshenboim, Phys. Rep. 422, 1 (2005)

146. G.T. Bodwin, D.R. Yennie, M.A. Gregorio, Rev. Mod. Phys. 57, 723 (1985)

147. G.S. Adkins, R.N. Fell, Phys. Rev. A 60, 4461 (1999)

148. H.A. Bethe, E.E. Salpeter, Quantum mechanics of oneand two-electron atoms (Springer, Berlin, 1957)

149. W. Bernreuther, U. Löw, J.P. Ma, O. Nachtmann, Z. Phys. C: Part. Fields 41, 143 (1988)

150. K. Pachucki, S.G. Karshenboim, Phys. Rev. Lett. 80, 2101 (1998)

151. A. Czarnecki, K. Melnikov, A. Yelkhovsky, Phys. Rev. Lett. 82, 311 (1999)

152. J. Zatorski, Phys. Rev. A 78, 032103 (2008)

153. M. Baker, P. Marquard, A.A. Penin, J. Piclum, M. Steinhauser, Phys. Rev. Lett. 112, 120407 (2014)

154. G.S. Adkins, C. Parsons, M.D. Salinger, R. Wang, R.N. Fell, Phys. Rev. A 90, 042502 (2014)

155. G.S. Adkins, L.M. Tran, R. Wang, Phys. Rev. A 93, 052511 (2016)

156. R. Ley, in 9th International Workshop on Slow Positron Beam Techniques for Solids and Surfaces, Appl. Surf. Sci. 194, 301 (2002)

157. R. Pohl et al., Nature 466, 213 (2010)

158. A. Antognini et al., Science 339, 417 (2013)

159. R. Pohl, R. Gilman, G.A. Miller, K. Pachucki, Annu. Rev. Nucl. Part. Sci. 63, 175 (2013)

160. A. Osterwalder, F. Merkt, Phys. Rev. Lett. 82, 1831 (1999)

161. F. Nez et al., Phys. Rev. Lett. 69, 2326 (1992)

162. F. Biraben, J.C. Garreau, L. Julien, M. Allegrini, Phys. Rev. Lett. 62, 621 (1989)

163. D.W. Gidley, P.W. Zitzewitz, K.A. Marko, A. Rich, Phys. Rev. Lett. 37, 729 (1976)

164. A.G. Cohen, A.D. Rjula, S.L. Glashow, Astrophys. J. 495, 539 (1998)

165. W.A. Perkins, Mod. Phys. Lett. A 30, 1550157 (2015)
166. M. Fukugita, T. Yanagida, Phys. Lett. B 174, 45 (1986)

167. A.D. Sakharov, JETP Lett. 5, 24 (1967)

168. G.C. Branco, R. González Felipe, F.R. Joaquim, Rev. Mod. Phys. 84, 515 (2012)

169. G. Chardin, Nucl. Phys. A 558, 477 (1993)

170. M.M. Nieto, T. Goldman, Phys. Rep. 205, 221 (1991)

171. C. Foot, Atomic physics, Oxford master series in physics (Oxford University Press, New York, 2005)

172. R. Ley, D. Hagena, D. Weil, G. Werth, W. Arnold, H. Schneider, Hyperfine Interact. 89, 327 (1994)

173. V.B. Berestetski, J. Exp. Theor. Phys. (U.S.S.R.) 19, 1130 (1949)

174. R.A. Ferrell, Phys. Rev. 84, 858 (1951)

175. T.F. Gallagher, Rydberg atoms (Cambridge University Press, Cambridge, UK, 1994)

176. F. Merkt, R.N. Zare, J. Chem. Phys. 101, 3495 (1994)

177. L. Michel, Il Nuovo Cimento (1943-1954) 10, 319 (1953)

178. C.N. Yang, Phys. Rev. 77, 242 (1950)

179. L. Wolfenstein, D.G. Ravenhall, Phys. Rev. 88, 279 (1952)

180. S. Shimizu, T. Mukoyama, Y. Nakayama, Phys. Rev. 173 , 405 (1968)

181. H. Mazaki, M. Nishi, S. Shimizu, Phys. Rev. 171, 408 (1968)

182. J.C. Palathingal, P. Asoka-Kumar, K.G. Lynn, Y. Posada, X.Y. Wu, Phys. Rev. Lett. 67, 3491 (1991)

183. A.W. Hunt, D.B. Cassidy, F.A. Selim, R. Haakenaasen, T.E. Cowan, R.H. Howell, K.G. Lynn, J.A. Golovchenko, Nature 402, 157 (1999)

184. A.I. Alekseev, Sov. Phys. JETP 7, 826 (1958)

185. A.I. Alekseev, Sov. Phys. JETP 9, 1312 (1959)

186. P.A.M. Dirac, Math. Proc. Camb. Philos. Soc. 26, 361 (1930)

187. P. Wallyn, W.A. Mahoney, P. Durouchoux, C. Chapuis, APJ 465, 473 (1996)

188. J. Shapiro, G. Breit, Phys. Rev. 113, 179 (1959)

189. A.H. Al-Ramadhan, D.W. Gidley, Phys. Rev. Lett. 72, 1632 (1994)

190. G.S. Adkins, R.N. Fell, J. Sapirstein, Ann. Phys. 295, $136(2002)$

191. P.G. Coleman, T.C. Griffith, J. Phys. B: At. Mol. Phys. 6, $2155(1973)$

192. D.W. Gidley, A. Rich, P.W. Zitzewitz, D.A.L. Paul, Phys. Rev. Lett. 40, 737 (1978)

193. T.C. Griffith, G.R. Heyland, K.S. Lines, T.R. Twomey, J. Phys. B: At. Mol. Phys. 11, L743 (1978)

194. D. Gidley, P. Zitzewitz, Phys. Lett. A 69, 97 (1978)

195. S. Asai, S. Orito, N. Shinohara, Phys. Lett. B 357, 475 (1995)

196. Y. Kataoka, S. Asai, T. Kobayashi, Phys. Lett. B 671, $219(2009)$

197. T. Namba, Progr. Theor. Exp. Phys. 2012, 04 D003 (2012)

198. S. DeBenedetti, R.T. Siegel, Phys. Rev. 94, 955 (1954)

199. S.C. Pevovar, M.H. Weber, K.G. Lynn, Phys. Status Solidi (c) 4, 3447 (2007)

200. M. Chin, D. Seweryniak, M. Alkhorayef, N. Spyrou, in Proceedings of the 8th International Conference on Position Sensitive Detectors, Nucl. Instrum. Methods Phys. Res. Sect. A: Accel. Spectrom. Detect. Assoc. Equip. 604, 331 (2009)

201. R.M. Drisko, Phys. Rev. 102, 1542 (1956)

202. R.S. Conti, S. Hatamian, A. Rich, Phys. Rev. A 33, 3495 (1986) 
203. D.C. Liu, W.K. Roberts, Phys. Rev. Lett. 16, 67 (1966) 204. K. Marko, A. Rich, Phys. Rev. Lett. 33, 980 (1974)

205. S. Adachi, M. Chiba, T. Hirose, S. Nagayama, Y. Nakamitsu, T. Sato, T. Yamada, Phys. Rev. Lett. 65, 2634 (1990)

206. A.P. Mills Jr., D.M. Zuckerman, Phys. Rev. Lett. 64, 2637 (1990)

207. J. Yang, M. Chiba, R. Hamatsu, T. Hirose, T. Matsumoto, J. Yu, Phys. Rev. A 54, 1952 (1996)

208. T. Matsumoto, M. Chiba, R. Hamatsu, T. Hirose, J. Yang, J. Yu, Phys. Rev. A 54, 1947 (1996)

209. T. Mitsui, K. Maki, S. Asai, Y. Ishisaki, R. Fujimoto, N. Muramoto, T. Sato, Y. Ueda, Y. Yamazaki, S. Orito, Europhys. Lett. 33, 111 (1996)

210. A. Badertscher, P. Crivelli, M. Felcini, S. Gninenko, N. Goloubev, P. Ndlec, J. Peigneux, V. Postoev, A. Rubbia, D. Sillou, Phys. Lett. B 542, 29 (2002)

211. P.A. Vetter, S.J. Freedman, Phys. Rev. A 66, 052505 (2002)

212. M. Skalsey, J. Van House, Phys. Rev. Lett. 67, 1993 (1991)

213. T. Yamazaki, T. Namba, S. Asai, T. Kobayashi, Phys. Rev. Lett. 104, 083401 (2010)

214. P.A. Vetter, S.J. Freedman, Phys. Rev. Lett. 91, 263401 (2003)

215. B.K. Arbic, S. Hatamian, M. Skalsey, J. Van House, W. Zheng, Phys. Rev. A 37, 3189 (1988)

216. T. Mitsui, R. Fujimoto, Y. Ishisaki, Y. Ueda, Y. Yamazaki, S. Asai, S. Orito, Phys. Rev. Lett. 70, 2265 (1993)

217. A. Badertscher, P. Crivelli, W. Fetscher, U. Gendotti, S.N. Gninenko, V. Postoev, A. Rubbia, V. Samoylenko, D. Sillou, Phys. Rev. D 75, 032004 (2007)

218. P. Crivelli, A. Belov, U. Gendotti, S. Gninenko, A. Rubbia, J. Instrum. 5, P08001 (2010)

219. A. Pokraka, A. Czarnecki, Phys. Rev. D 94, 113012 (2016)

220. S.N. Gninenko, N.V. Krasnikov, A. Rubbia, Phys. Rev. D 67, 075012 (2003)

221. A. Friedland, M. Giannotti, Phys. Rev. Lett. 100, 031602 (2008)

222. Y. Liao, Phys. Rev. D 76, 056006 (2007)

223. S. Asai, S. Orito, K. Yoshimura, T. Haga, Phys. Rev. Lett. 66, 2440 (1991)

224. S. Asai, K. Shigekuni, T. Sanuki, S. Orito, Phys. Lett. B 323, 90 (1994)

225. L.B. Okun, Phys. Usp. 50, 380 (2007)

226. S.V. Demidov, D.S. Gorbunov, A.A. Tokareva, Phys. Rev. D 85, 015022 (2012)

227. W. Demtröder, in Laser spectroscopy, 3rd edn. (Springer, New York, 2003)

228. G. Bearman, A. Mills, Phys. Lett. A 56, 350 (1976)

229. G. Laricchia, M. Charlton, G. Clark, T. Griffith, Phys. Lett. A 109, 97 (1985)

230. A.J. Garner, G. Laricchia, A. Zen, J. Phys. B: At. Mol. Opt. Phys. 29, 5961 (1996)

231. G. Laricchia, S.A. Davies, M. Charlton, T.C. Griffith, J. Phys. E: Sci. Instrum. 21, 886 (1988)

232. S.J. Brawley, S.E. Fayer, M. Shipman, G. Laricchia, Phys. Rev. Lett. 115, 223201 (2015)

233. K.G. Lynn, J. Phys. C: Solid State Phys. 12, L435 (1979)

234. A.P. Mills Jr., L. Pfeiffer, P.M. Platzman, Phys. Rev. Lett. 51, 1085 (1983)
235. A.P. Mills Jr., E.D. Shaw, M. Leventhal, R.J. Chichester, D.M. Zuckerman, Phys. Rev. B 44, 5791 (1991)

236. M. Eldrup, A. Vehanen, P.J. Schultz, K.G. Lynn, Phys. Rev. B 32, 7048 (1985)

237. S. Curry, A. Schawlow, Phys. Lett. A 37, 5 (1971)

238. P. Sferlazzo, S. Berko, K.F. Canter, Phys. Rev. B 35, 5315 (1987)

239. Y. Nagashima, Y. Morinaka, T. Kurihara, Y. Nagai, T. Hyodo, T. Shidara, K. Nakahara, Phys. Rev. B 58, 12676 (1998)

240. S. Mariazzi, P. Bettotti, S. Larcheri, L. Toniutti, R.S. Brusa, Phys. Rev. B 81, 235418 (2010)

241. S. Mariazzi, P. Bettotti, R.S. Brusa, Phys. Rev. Lett. 104, $243401(2010)$

242. A.P. Mills Jr. W.S. Crane, Phys. Rev. A 31, 593 (1985)

243. M.R. Poulsen, M. Charlton, J. Chevallier, B.I. Deutch, L.V. Jorgensen, G. Laricchia, J. Phys.: Condens. Matter 3, 2849 (1991)

244. S.J. Tao, Appl. Phys. 10, 67 (1976)

245. G.W. Ford, L.M. Sander, T.A. Witten, Phys. Rev. Lett. 36, 1269 (1976)

246. L. Liszkay et al., Appl. Phys. Lett. 92, 063114 (2008)

247. P. Crivelli, U. Gendotti, A. Rubbia, L. Liszkay, P. Perez, C. Corbel, Phys. Rev. A 81, 052703 (2010)

248. D.W. Gidley, W.E. Frieze, T.L. Dull, A.F. Yee, E.T. Ryan, H.M. Ho, Phys. Rev. B 60, R5157 (1999)

249. D.W. Gidley, H.G. Peng, R.S. Vallery, Annu. Rev. Mater. Res. 36, 49 (2006)

250. J. Callaway, Phys. Rev. 116, 1140 (1959)

251. A. Held, S. Kahana, Can. J. Phys. 42, 19081964

252. A.P. Mills Jr., C.A. Murray, Appl. Phys. 21, 323 (1980)

253. C.H. Hodges, M.J. Stott, Phys. Rev. B 7, 73 (1973)

254. D.W. Gidley, W.E. Frieze, Phys. Rev. Lett. 60, 1193 (1988)

255. A.P. Mills Jr. E.D. Shaw, R.J. Chichester, D.M. Zuckerman, Phys. Rev. B 40, 8616 (1989)

256. M.J. Puska, R.M. Nieminen, Rev. Mod. Phys. 66, 841 (1994)

257. R.H. Howell, I.J. Rosenberg, M.J. Fluss, R.E. Goldberg, R.B. Laughlin, Phys. Rev. B 35, 5303 (1987)

258. A. Damascelli, Z. Hussain, Z.X. Shen, Rev. Mod. Phys. 75, 473 (2003)

259. R. Ley, K.D. Niebling, R. Schwarz, G. Werth, J. Phys. B: At. Mol. Opt. Phys. 23, 19151990

260. D.C. Schoepf, S. Berko, K.F. Canter, P. Sferlazzo, Phys. Rev. A 45, 1407 (1992)

261. T.D. Steiger, R.S. Conti, Phys. Rev. A 45, 2744 (1992)

262. D.J. Day, M. Charlton, G. Laricchia, J. Phys. B: At. Mol. Opt. Phys. 34, 3617 (2001)

263. K.F. Canter, A.P. Mills Jr., S. Berko, Phys. Rev. Lett. 34, 177 (1975)

264. A.P. Mills Jr., S. Berko, K.F. Canter, Phys. Rev. Lett. 34, 1541 (1975)

265. S. Hatamian, R.S. Conti, A. Rich, Phys. Rev. Lett. 58, 1833 (1987)

266. D. Hagena, R. Ley, D. Weil, G. Werth, W. Arnold, H. Schneider, Phys. Rev. Lett. 71, 2887 (1993)

267. R. Paulin, R. Ripon, W. Brandt, Phys. Rev. Lett. 31, 1214 (1973)

268. C. Hodges, M. Stott, Solid State Commun. 12, 1153 (1973)

269. R. Nieminen, M. Manninen, Solid State Commun. 15, 403 (1974) 
270. K.G. Lynn, W.E. Frieze, P.J. Schultz, Phys. Rev. Lett. 52, 1137 (1984)

271. A.P. Mills Jr., Phys. Rev. Lett. 41, 1828 (1978)

272. A.P. Mills Jr., Solid State Commun. 31, 623 (1979)

273. K.G. Lynn, Phys. Rev. Lett. 43, 391 (1979)

274. K.G. Lynn, D.O. Welch, Phys. Rev. B 22, 99 (1980)

275. S. Chu, A.P. Mills Jr., C.A. Murray, Phys. Rev. B 23, 2060 (1981)

276. M. Weinelt, M. Kutschera, T. Fauster, M. Rohlfing, Phys. Rev. Lett. 92, 126801 (2004)

277. M.P. Petkov, C.L. Wang, M.H. Weber, K.G. Lynn, K.P. Rodbell, J. Phys. Chem. B 107, 2725 (2003)

278. H.G. Peng, R.S. Vallery, M. Liu, M. Skalsey, D.W. Gidley, Colloids Surf. A: Physicochem. Eng. Asp. 300, 154 (2007)

279. C.G. Fischer, M.H. Weber, C.L. Wang, S.P. McNeil, K.G. Lynn, Phys. Rev. B 71, 180102 (2005)

280. C. He, T. Ohdaira, N. Oshima, M. Muramatsu, A. Kinomura, R. Suzuki, T. Oka, Y. Kobayashi, Phys. Rev. B 75, 195404 (2007)

281. C. He, T. Oka, Y. Kobayashi, N. Oshima, T. Ohdaira, A. Kinomura, R. Suzuki, Appl. Phys. Lett. 91, 024102 (2007)

282. L. Liszkay et al., Appl. Surf. Sci. 255, 187 (2008)

283. L. Liszkay et al., Appl. Phys. Lett. 95, 124103 (2009)

284. L. Liszkay et al., New J. Phys. 14, 065009 (2012)

285. N.K. Plugotarenko, V.V. Petrov, V.A. Ivanetz, V.A. Smirnov, Glass Phys. Chem. 37, 590 (2011)

286. S. Mariazzi, A. Salemi, R.S. Brusa, Phys. Rev. B 78, 085428 (2008)

287. D.B. Cassidy, S.H.M. Deng, R.G. Greaves, T. Maruo, N. Nishiyama, J.B. Snyder, H.K.M. Tanaka, A.P. Mills Jr., Phys. Rev. Lett. 95, 195006 (2005)

288. D.B. Cassidy, A.P. Mills Jr., Phys. Rev. Lett. 100, 013401 (2008)

289. D.B. Cassidy, K.T. Yokoyama, S.H.M. Deng, D.L. Griscom, H. Miyadera, H.W.K. Tom, C.M. Varma, A.P. Mills Jr., Phys. Rev. B 75, 085415 (2007)

290. D.B. Cassidy, A.P. Mills Jr., Phys. Rev. Lett. 107, 213401 (2011)

291. Z. Yu, J.D. McGervey, A.M. Jamieson, R. Simha, Macromolecules 28, 6268 (1995)

292. A.H. Baugher, W.J. Kossler, K.G. Petzinger, Macromolecules 29, 7280 (1996)

293. S.M. Kim, W.J.L. Buyers, J. Phys. C: Solid State Phys. 11, 101 (1978)

294. Y. Nagashima et al., Phys. Rev. A 52, 258 (1995)

295. Y. Zhou, J. Li, C. Yin, W. Mao, J. Wang, C. He, Phys. Rev. A 94, 022513 (2016)

296. N. Suzuki, T. Oonishi, T. Hyodo, T. Chang, Appl. Phys. A 74, 791 (2002)

297. C.J. Edwardson, M.D.W. Grogan, T.A. Birks, P.G. Coleman, J. Phys.: Conf. Ser. 262, 012018 (2011)

298. Y. Zhou, W. Mao, Q. Li, J. Wang, C. He, Chem. Phys. 459, 81 (2015)

299. W. Brandt, G. Coussot, R. Paulin, Phys. Rev. Lett. 23, $522(1969)$

300. A. Greenberger, A. Mills, A. Thompson, S. Berko, Phys. Lett. A 32, 72 (1970)

301. D. Dutta, J.I. Feldblyum, D.W. Gidley, J. Imirzian, M. Liu, A.J. Matzger, R.S. Vallery, A.G. Wong-Foy, Phys. Rev. Lett. 110, 197403 (2013)
302. P. Crivelli, D. Cooke, B. Barbiellini, B.L. Brown, J.I. Feldblyum, P. Guo, D.W. Gidley, L. Gerchow, A.J. Matzger, Phys. Rev. B 89, 241103 (2014)

303. Z. Wang, S.M. Cohen, Chem. Soc. Rev. 38, 1315 (2009)

304. M. O'Keeffe, Chem. Soc. Rev. 38, 1215 (2009)

305. J.L. Rowsell, O.M. Yaghi, Microporous Mesoporous Mater. 73, 3 (2004)

306. A.P. Mills Jr. R.J. Wilson, Phys. Rev. A 26, 490 (1982)

307. J. Algers, P. Sperr, W. Egger, G. Kögel, F.H.J. Maurer, Phys. Rev. B 67, 125404 (2003)

308. S.L. Andersen, R.R. Johansen, J.B. Overgaard, J.K. Mortensen, K.K. Andersen, H.D. Thomsen, M.D. Lund, J. Chevallier, H. Knudsen, U.I. Uggerhøj, Eur. Phys. J. D 68, $124(2014)$

309. O. Halpern, Phys. Rev. 94, 904 (1954)

310. S. Debenedetti, H.C. Corben, Annu. Rev. Nucl. Sci. 4 191 (1954)

311. M.A. Stroscio, Phys. Rep. 22, 215 (1975)

312. A. Rich, Phys. Rev. A 23, 2747 (1981)

313. A.P. Mills Jr., Phys. Rev. A 27, 262 (1983)

314. A.P. Mills Jr., Phys. Rev. A 41, 502 (1990)

315. G. Feinberg, A. Rich, J. Sucher, Phys. Rev. A 41, 3478 (1990)

316. P.B. Schwinberg, R.S. Van Dyck, H.G. Dehmelt, Phys. Rev. Lett. 47, 1679 (1981)

317. R.S. Van Dyck, P.B. Schwinberg, H.G. Dehmelt, Phys. Rev. Lett. 59, 26 (1987)

318. D. Hanneke, S. Fogwell, G. Gabrielse, Phys. Rev. Lett. 100, 120801 (2008)

319. D. Hanneke, S. Fogwell Hoogerheide, G. Gabrielse, Phys. Rev. A 83, 052122 (2011)

320. T. Aoyama, M. Hayakawa, T. Kinoshita, M. Nio, Phys. Rev. Lett. 109, 111807 (2012)

321. G. Breit, I.I. Rabi, Phys. Rev. 38, 2082 (1931)

322. V.W. Hughes, S. Marder, C.S. Wu, Phys. Rev. 106, 934 (1957)

323. T. Yamazaki, A. Miyazaki, T. Suehara, T. Namba, S. Asai, T. Kobayashi, H. Saito, I. Ogawa, T. Idehara, S. Sabchevski, Phys. Rev. Lett. 108, 253401 (2012)

324. Y. Tatematsu, Y. Yamaguchi, T. Idehara, T. Kawase, R. Ichioka, I. Ogawa, T. Saito, T. Fujiwara, J. Infrared Millim. Terahertz Waves 35, 169 (2014)

325. A. Miyazaki, T. Yamazaki, T. Suehara, T. Namba, S. Asai, T. Kobayashi, H. Saito, T. Idehara, I. Ogawa, Y. Tatematsu, J. Infrared Millim. Terahertz Waves 35, 91 (2014)

326. M. Glyavin, V. Manuilov, T. Idehara, Phys. Plasmas 20, 123303 (2013)

327. K.S. Shah, J. Glodo, M. Klugerman, W.W. Moses, S.E. Derenzo, M.J. Weber, IEEE Trans. Nucl. Sci. 50, 2410 (2003)

328. A. Alonso, B. Cooper, A. Deller, D. Cassidy, Nucl. Instrum. Methods Phys. Res Sect. A: Accel. Spectrom. Detect. Assoc. Equip. 828, 163 (2016)

329. N.C. Hurst, J.R. Danielson, C.M. Surko, Phys. Plasmas 22, 073503 (2015)

330. S.M. Curry, Phys. Rev. A 7, 447 (1973)

331. M.L. Lewis, V.W. Hughes, Phys. Rev. A 8, 625 (1973)

332. H. Grotch, R. Kashuba, Phys. Rev. A 7, 78 (1973)

333. C.D. Dermer, J.C. Weisheit, Phys. Rev. A 40, 5526 (1989)

334. A. Raspini, Int. J. Theor. Phys. 28, 1359 (1989) 
335. J.M. Anthony, K.J. Sebastian, Phys. Rev. A 49, 192 (1994)

336. S.D. Hogan, Phys. Rev. A 87, 063423 (2013)

337. R.J. Damburg, V.V. Kolosov, in Rydberg states of atoms and molecules, edited by R.F. Stebbings, F.B. Dunning (Cambridge University Press, Cambridge, 1983), pp. $31-72$

338. S.D. Hogan, EPJ Tech. Instrum. 3, 1 (2016)

339. J.E. McClintock, APJ 282, 291 (1984)

340. S.C. Ellis, J. Bland-Hawthorn, Astrophys. J. 707, 457 (2009)

341. N. Prantzos et al., Rev. Mod. Phys. 83, 1001 (2011)

342. E.D. Bloom et al., Phys. Rev. Lett. 23, 930 (1969)

343. T.W. Hänsch, M.D. Levenson, A.L. Schawlow, Phys. Rev. Lett. 26, 946 (1971)

344. M.S. Fee, K. Danzmann, S. Chu, Phys. Rev. A 45, 4911 (1992)

345. A.P. Mills Jr., Appl. Phys. 22, 273 (1980)

346. A.P. Mills Jr., E.D. Shaw, R.J. Chichester, D.M. Zuckerman, Rev. Sci. Instrum. 60, 825 (1989)

347. R.H. Howell, R.A. Alvarez, M. Stanek, Appl. Phys. Lett. 40, $751(1982)$

348. F. Ebel, W. Faust, C. Hahn, S. Langer, M. Rckert, H. Schneider, A. Singe, I. Tobehn, Nucl. Instrum. Methods Phys. Res Sect. A: Accel. Spectrom. Detect. Assoc. Equip. 272, 626 (1988)

349. K. Wada et al., J. Phys.: Conf. Ser. 443, 012082 (2013)

350. M. Jungmann, J. Haeberle, R. Krause-Rehberg, W. Anwand, M. Butterling, A. Wagner, J.M. Johnson, T.E. Cowan, J. Phys.: Conf. Ser. 443, 012088 (2013)

351. J.H. Malmberg, J.S. deGrassie, Phys. Rev. Lett. 35, 577 (1975)

352. X.P. Huang, F. Anderegg, E.M. Hollmann, C.F. Driscoll, T.M. O'Neil, Phys. Rev. Lett. 78, 875 (1997)

353. R.G. Greaves, C.M. Surko, Phys. Rev. Lett. 85, 1883 (2000)

354. J.R. Danielson, C.M. Surko, Phys. Rev. Lett. 94, 035001 (2005)

355. J.R. Danielson, C.M. Surko, T.M. O’Neil, Phys. Rev. Lett. 99, 135005 (2007)

356. D.B. Cassidy, S.H.M. Deng, R.G. Greaves, A.P. Mills Jr., Rev. Sci. Instrum. 77, 073106 ) (2006)

357. B.S. Cooper, A.M. Alonso, A. Deller, T.E. Wall, D.B. Cassidy, Rev. Sci. Instrum. 86, 103101 (2015)

358. S. Townrow, P.G. Coleman, Rev. Sci. Instrum. 84, 103908 (2013)

359. D.B. Cassidy, A.P. Mills Jr., Nucl. Instrum. Methods A 580, 1338 (2007)

360. D.B. Cassidy, A.P. Mills Jr., Nature 449, 195 (2007)

361. D.B. Cassidy, H.W.K. Tom, A.P. Mills Jr., AIP Conf. Proc. 1037, 66 (2008)

362. D.B. Cassidy, V.E. Meligne, A.P. Mills Jr., Phys. Rev. Lett. 104, 173401 (2010)

363. M.E. Phelps, Annu. Rev. Nucl. Part. Sci. 52, 303 (2002)

364. M. Tuomisaari, R.H. Howell, T. McMullen, Phys. Rev. B 40, 2060 (1989)

365. D.B. Cassidy, S.H.M. Deng, H.K.M. Tanaka, A.P. Mills Jr., Appl. Phys. Lett. 88, 194105 ( 3) (2006)

366. R.G. Greaves, J. Moxom, AIP Conf. Proc. 692, 140 (2003)

367. R.G. Greaves, J.M. Moxom, Phys. Plasmas 15, 072304 (2008)

368. C.A. Isaac, C.J. Baker, T. Mortensen, D.P. van der Werf, M. Charlton, Phys. Rev. Lett. 107, 033201 (2011)
369. T. Hyodo, T. Nakayama, H. Saito, F. Saito, K. Wada, Phys. Status Solidi (c) 6, 2497 (2009)

370. H. Saito, T. Hyodo, Phys. Rev. Lett. 97, 253402 (2006)

371. N. Shinohara, N. Suzuki, T. Chang, T. Hyodo, Phys. Rev. A 64, 042702 (2001)

372. S.Y. Chuang, S.J. Tao, Phys. Rev. A 9, 989 (1974)

373. T.W. Hänsch, I.S. Shahin, A.L. Schawlow, Phys. Rev. Lett. 27, 707 (1971)

374. R.A. McFarlane, W.R. Bennett Jr., W.E. Lamb Jr., Appl. Phys. Lett. 2, 189 (1963)

375. W.E. Lamb, Phys. Rev. 134, A1429 (1964)

376. A.P. Mills Jr. G.H. Bearman, Phys. Rev. Lett. 34, 246 (1975)

377. M.W. Ritter, P.O. Egan, V.W. Hughes, K.A. Woodle, Phys. Rev. A 30, 1331 (1984)

378. V.G. Baryshevsky, O.N. Metelitsa, V.V. Tikhomirov, J. Phys. B: At. Mol. Opt. Phys. 22, 2835 (1989)

379. V. Baryshevsky, O. Metelitsa, V. Tikhomirov, S. Andrukhovich, A. Berestov, B. Martsinkevich, E. Rudak, Phys. Lett. A 136, 428 (1989)

380. I. Bondarev, S. Kuten, Phys. Lett. A 154, 154 (1991)

381. S. Fan, C. Beling, S. Fung, Phys. Lett. A 216, 129 (1996)

382. E. Ivanov, I. Vata, S. Teodorian, I. Rusen, N. Stefan, in Proceedings of the Fourth International Conference on Elementary Processes in Atomic Systems, Nucl. Instrum. Methods Phys. Res. Sect. B: Beam Interact. Mater. At. 267, 347 (2009)

383. Y. Sasaki, A. Miyazaki, A. Ishida, T. Namba, S. Asai, T. Kobayashi, H. Saito, K. Tanaka, A. Yamamoto, Phys. Lett. B 697, 121 (2011)

384. E.P. Liang, C.D. Dermer, Opt. Commun. 65, 419 (1988)

385. A.P. Mills Jr., Positron Solid state physics, in Proceedings of the International School of Physics 'Enrico Fermi' Course LXXXIII "Positron Solid-state Physics", edited by W. Brandt, A. Dupasquier (IOS Press, Amsterdam, 1983), pp. $77-187$

386. H.O. Anger, Rev. Sci. Instrum. 29, 27 (1958)

387. R.N. West, J. Mayers, P.A. Walters, J. Phys. E 14, 478 (1981)

388. Y. Nagashima, T. Hyodo, K. Fujiwara, A. Ichimura, J. Phys. B 31, 329 (1998)

389. T. Chang, M. Xu, X. Zeng, Phys. Lett. A 126, 189 (1987)

390. M. Skalsey, J.J. Engbrecht, R.K. Bithell, R.S. Vallery, D.W. Gidley, Phys. Rev. Lett. 80, 3727 (1998)

391. M. Skalsey, J.J. Engbrecht, R.K. Bithell, R.S. Vallery, D.W. Gidley, Phys. Rev. Lett. 80, 3727 (1998)

392. H. Stoll, M. Koch, K. Maier, J. Major, Nucl. Instrum. Methods Phys. Res. Sect. B: Beam Interact. Mater. At. 56, 582 (1991)

393. H. Stoll, M. Koch, U. Lauff, K. Maier, J. Major, H. Schneider, A. Seeger, A. Siegle, Appl. Surf. Sci. 85, 17 (1995)

394. J. Mitroy, S.A. Novikov, Phys. Rev. Lett. 90, 183202 (2003)

395. K. Shibuya, Y. Kawamura, H. Saito, Phys. Rev. A 88, 042517 (2013)

396. K. Shibuya, T. Nakayama, H. Saito, T. Hyodo, Phys. Rev. A 88, $012511(2013)$

397. A. Sommerfeld, H. Welker, Ann. Phys. 424, 56 (1938)

398. P.W. Fowler, Mol. Phys. 53, 865 (1984)

399. J.P. Connerade, Phys. Scr. 68, C25 (2003)

400. V. Dolmatov, A. Baltenkov, J.P. Connerade, S. Manson, Radiat. Phys. Chem. 70, 417 (2004)

401. K.G. Dvoyan, Physica B 407, 131 (2012) 
402. J.A. Ludlow, T.G. Lee, Phys. Rev. A 91, 032507 (2015)

403. R. Brown, Q. Prigent, A.R. Swann, G.F. Gribakin, Phys. Rev. A 95, 032705 (2017)

404. S. Villalba, H. Failache, A. Laliotis, L. Lenci, S. Barreiro, A. Lezama, Opt. Lett. 38, 193 (2013)

405. T. Svensson, E. Adolfsson, M. Lewander, C.T. Xu, S. Svanberg, Phys. Rev. Lett. 107, 143901 (2011)

406. R.H. Dicke, Phys. Rev. 89, 472 (1953)

407. D.G. Green, G.F. Gribakin, Phys. Rev. Lett. 106, 209301 (2011)

408. F. Guillemot, A. Brunet-Bruneau, E. Bourgeat-Lami, T. Gacoin, E. Barthel, J.P. Boilot, Chem. Mater. 22, 2822 (2010)

409. K. Wada, T. Hyodo, J. Phys.: Conf. Ser. 443, 012003 (2013)

410. K. Ito, H. Nakanishi, Y. Ujihira, J. Phys. Chem. B 103, 4555 (1999)

411. G. Dufour, D. Cassidy, P. Crivelli, P. Debu, A. Lambrecht, V. Nesvizhevsky, S. Reynaud, A. Voronin, T. Wall, Adv. High Energy Phys. 2015, 379642 (2015)

412. P.F. Barker, M. Charlton, New J. Phys. 14, 045005 (2012)

413. J. Estrada, T. Roach, J.N. Tan, P. Yesley, G. Gabrielse, Phys. Rev. Lett. 84, 859 (2000)

414. C.J. Baker, D.P. van der Werf, D.C.S. Beddows, P.R. Watkeys, C.A. Isaac, S.J. Kerrigan, M. Charlton, H.H. Telle, J. Phys. B: At. Mol. Opt. Phys. 41, 245003 (2008)

415. D.P. van der Werf, C.J. Baker, D.C.S. Beddows, P.R. Watkeys, C.A. Isaac, S.J. Kerrigan, M. Charlton, H.H. Telle, J. Phys.: Conf. Ser. 199, 012005 (2010)

416. C.H. Storry et al. (ATRAP Collaboration), Phys. Rev. Lett. 93, 263401 (2004)

417. A. Speck, C. Storry, E. Hessels, G. Gabrielse, Phys. Lett. B 597, 257 (2004)

418. M. Charlton, Phys. Lett. A 143, 143 (1990)

419. A.S. Kadyrov, I. Bray, M. Charlton, I.I. Fabrikant, Nat. Commun. 8, 1544 (2017)

420. T.E. Wall, D.B. Cassidy, S.D. Hogan, Phys. Rev. A 90, $053430(2014)$

421. J. Sjakste, A.G. Borisov, J.P. Gauyacq, Phys. Rev. A 73, 042903 (2006)

422. S. Wethekam, H.R. Dunham, J.C. Lancaster, F.B. Dunning, Phys. Rev. A 73, 032903 (2006)

423. Y. Pu, D.D. Neufeld, F.B. Dunning, Phys. Rev. A 81, 042904 (2010)

424. Y. Pu, F.B. Dunning, Phys. Rev. A 88, 012901 (2013)

425. S.B. Hill, C.B. Haich, Z. Zhou, P. Nordlander, F.B. Dunning, Phys. Rev. Lett. 85, 5444 (2000)

426. E. So, M. Dethlefsen, M. Ford, T.P. Softley, Phys. Rev. Lett. 107, 093201 (2011)

427. J.A. Gibbard, M. Dethlefsen, M. Kohlhoff, C.J. Rennick, E. So, M. Ford, T.P. Softley, Phys. Rev. Lett. 115, 093201 (2015)

428. T. Thiele, J. Deiglmayr, M. Stammeier, J.A. Agner, H. Schmutz, F. Merkt, A. Wallraff, Phys. Rev. A 92, 063425 (2015)

429. K.S. Chan, M. Siercke, C. Hufnagel, R. Dumke, Phys. Rev. Lett. 112, 026101 (2014)

430. J.W. Humberston, M. Charlton, F.M. Jacobson, B.I. Deutch, J. Phys. B: At. Mol. Phys. 20, L25 (1987)

431. D.B. Cassidy, J.P. Merrison, M. Charlton, J. Mitroy, G. Ryzhikh, J. Phys. B: At. Mol. Opt. Phys. 32, 1923 (1999)

432. B.I. Deutch, M. Charlton, M.H. Holzscheiter, P. Hvelplund, L.V. Jørgensen, H. Knudsen, G. Laricchia,
J.P. Merrison, M.R. Poulsen, Hyperfine Interact. 76, 151 (1993)

433. J.P. Merrison, H. Bluhme, J. Chevallier, B.I. Deutch, P. Hvelplund, L.V. Jørgensen, H. Knudsen, M.R. Poulsen, M. Charlton, Phys. Rev. Lett. 78, 2728 (1997)

434. T. Halfmann, J. Koensgen, K. Bergmann, Meas. Sci. Technol. 11, 1510 (2000)

435. W.A. Bertsche, M. Charlton, S. Eriksson, New J. Phys. 19, $053020(2017)$

436. S.D. Hogan, F. Merkt, Phys. Rev. Lett. 100, 043001 (2008)

437. T.F. Gallagher, W.E. Cooke, Phys. Rev. Lett. 42, 835 (1979)

438. W.E. Cooke, T.F. Gallagher, Phys. Rev. A 21, 588 (1980)

439. W.P. Spencer, A.G. Vaidyanathan, D. Kleppner, T.W. Ducas, Phys. Rev. A 26, 1490 (1982)

440. E.J. Galvez, J.R. Lewis, B. Chaudhuri, J.J. Rasweiler, H. Latvakoski, F. De Zela, E. Massoni, H. Castillo, Phys. Rev. A 51, 4010 (1995)

441. E.J. Galvez, C.W. MacGregor, B. Chaudhuri, S. Gupta, E. Massoni, F. De Zela, Phys. Rev. A 55, 3002 (1997)

442. K.S. Lai, E.A. Hinds, Phys. Rev. Lett. 81, 2671 (1998)

443. X. Lu, Y. Sun, H. Metcalf, Phys. Rev. A 84, 033402 (2011)

444. V.D. Ovsiannikov, A. Derevianko, K. Gibble, Phys. Rev. Lett. 107, 093003 (2011)

445. S.D. Hogan, J.A. Agner, F. Merkt, T. Thiele, S. Filipp, A. Wallraff, Phys. Rev. Lett. 108, 063004 (2012)

446. J.P. Gordon, H.J. Zeiger, C.H. Townes, Phys. Rev. 99, $1264(1955)$

447. H.L. Bethlem, G. Berden, G. Meijer, Phys. Rev. Lett. 83, 1558 (1999)

448. H.L. Bethlem, F.M.H. Crompvoets, R.T. Jongma, S.Y.T. van de Meerakker, G. Meijer, Phys. Rev. A 65, 053416 (2002)

449. S.A. Rangwala, T. Junglen, T. Rieger, P.W.H. Pinkse, G. Rempe, Phys. Rev. A 67, 043406 (2003)

450. L.D. van Buuren, C. Sommer, M. Motsch, S. Pohle, M. Schenk, J. Bayerl, P.W.H. Pinkse, G. Rempe, Phys. Rev. Lett. 102, 033001 (2009)

451. M.T. Bell, T.P. Softley, Mol. Phys. 107, 99 (2009)

452. W.H. Wing, Phys. Rev. Lett. 45, 631 (1980)

453. T. Breeden, H. Metcalf, Phys. Rev. Lett. 47, 1726 (1981)

454. D. Townsend, A.L. Goodgame, S.R. Procter, S.R. Mackenzie, T.P. Softley, J. Phys. B: At. Mol. Opt. Phys. 34, 439 (2001)

455. Y. Yamakita, S.R. Procter, A.L. Goodgame, T.P. Softley, F. Merkt, J. Chem. Phys. 121, 1419 (2004)

456. E. Vliegen, F. Merkt, J. Phys. B.: At. Mol. Opt. Phys. 38, 1623 (2005)

457. E. Vliegen, P.A. Limacher, F. Merkt, Eur. Phys. J. D 40, 73 (2006)

458. E. Vliegen, H.J. Wörner, T.P. Softley, F. Merkt, Phys. Rev. Lett. 92, 033005 (2004)

459. E. Vliegen, F. Merkt, Phys. Rev. Lett. 97, 033002 (2006)

460. P. Lancuba, S.D. Hogan, Phys. Rev. A 88, 043427 (2013)

461. P. Allmendinger, J. Deiglmayr, J.A. Agner, H. Schmutz, F. Merkt, Phys. Rev. A 90, 043403 (2014)

462. E. Vliegen, S.D. Hogan, H. Schmutz, F. Merkt, Phys. Rev. A 76, 023405 (2007)

463. Ch. Seiler, S.D. Hogan, H. Schmutz, J.A. Agner, F. Merkt, Phys. Rev. Lett. 106, 073003 (2011)

464. S.D. Hogan, P. Allmendinger, H. Saßmannshausen, H. Schmutz, F. Merkt, Phys. Rev. Lett. 108, 063008 (2012) 
465. P. Lancuba, S.D. Hogan, Phys. Rev. A 90, 053420 (2014)

466. P. Lancuba, S.D. Hogan, J. Phys. B: At. Mol. Opt. Phys. 49, 074006 (2016)

467. S.D. Hogan, Ch. Seiler, F. Merkt, J. Phys. B.: At. Mol. Opt. Phys. 46, 045303 (2013)

468. C. Seiler, J.A. Agner, P. Pillet, F. Merkt, J. Phys. B: At. Mol. Opt. Phys. 49, 094006 (2016)

469. S.D. Hogan, C. Seiler, F. Merkt, Phys. Rev. Lett. 103, $123001(2009)$

470. Ch. Seiler, S.D. Hogan, F. Merkt, Phys. Chem. Chem. Phys. 13, 19000 (2011)

471. P. Allmendinger, J. Deiglmayr, O. Schullian, K. Höveler, J.A. Agner, H. Schmutz, F. Merkt, ChemPhysChem 17, 3596 (2016)

472. P. Allmendinger, J. Deiglmayr, K. Höveler, O. Schullian, F. Merkt, J. Chem. Phys. 145, 244316 (2016)

473. T. Rieger, T. Junglen, S.A. Rangwala, P.W.H. Pinkse, G. Rempe, Phys. Rev. Lett. 95, 173002 (2005)

474. C. Sommer, M. Motsch, S. Chervenkov, L.D. van Buuren, M. Zeppenfeld, P.W.H. Pinkse, G. Rempe, Phys. Rev. A 82, $013410(2010)$

475. A.L. Goodgame, T.P. Softley, J. Phys. B: At. Mol. Opt. Phys. 32, 4839 (1999)

476. A.R. Swann, D.B. Cassidy, A. Deller, G.F. Gribakin, Phys. Rev. A 93, 052712 (2016)

477. A.P. Mills Jr., M. Leventhal, Nucl. Instrum. Methods Phys. Res. Sect. B: Beam Interact. Mater. At. 192, 102 (2002)

478. L. Di Noto, S. Mariazzi, M. Bettonte, G. Nebbia, R. Brusa, Eur. Phys. J. D 66, 118 (2012)

479. H.O. Anger, D.H. Davis, Rev. Sci. Instrum. 35, 693 (1964)

480. G. Ferey, Chem. Soc. Rev. 37, 191 (2008)

481. H. Li, M. Eddaoudi, M. O'Keeffe, O.M. Yaghi, Nature 402, 276 (1999)

482. F. Bloch, Z. Phys. 52, 555 (1929)

483. W. Kohn, Phys. Rev. 115, 809 (1959)

484. P.D. Johnson, Angle-resolved photoemission (Springer International Publishing, Cham, 2014), pp. 1-32

485. D.M. Chen, S. Berko, K.F. Canter, K.G. Lynn, A.P. Mills, L.O. Roellig, P. Sferlazzo, M. Weinert, R.N. West, Phys. Rev. Lett. 58, 921 (1987)

486. J. Kuriplach, B. Barbiellini, Phys. Rev. B 89, 155111 (2014)

487. G.W. Erickson, J. Phys. Chem. Ref. Data 6, 831 (1977)

488. U. Jentschura, Ann. Phys. 326, 500 (2011)

489. B. Cagnac, M.D. Plimmer, L. Julien, F. Biraben, Rep. Prog. Phys. 57, 853 (1994)

490. T. Udem, R. Holzwarth, T.W. Hansch, Nature 416, 233 (2002)

491. C.G. Parthey et al., Phys. Rev. Lett. 107, 203001 (2011)

492. A. Beyer, J. Alnis, K. Khabarova, A. Matveev, C.G. Parthey, D.C. Yost, R. Pohl, T. Udem, T.W. Hänsch, N. Kolachevsky, Ann. Phys. 525, 671 (2013)

493. L.S. Vasilenko, V.P. Chebotaev, A.V. Shishaev, Sov. Phys. JETP Lett. 12, 113 (1970)

494. F. Biraben, B. Cagnac, G. Grynberg, Phys. Rev. Lett. 32, 643 (1974)

495. T.W. Hänsch, S.A. Lee, R. Wallenstein, C. Wieman, Phys. Rev. Lett. 34, 307 (1975)

496. M. Haas et al., Phys. Rev. A 73, 052501 (2006)

497. D.H. McIntyre, T.W. Hänsch, Phys. Rev. A 34, 4504 (1986)
498. K. Danzmann, M.S. Fee, S. Chu, Phys. Rev. A 39, 6072 (1989)

499. H. Higaki, K. Ito, K. Kira, H. Okamoto, Appl. Phys. Express 1, 066002 (2008)

500. E.D. Shaw, R.J. Chichester, A. La Porta, Proc. SPIE 1552, 14 (1991)

501. A.P. Mills Jr., Hyperfine Interact. 76, 233 (1993)

502. C. Wieman, T.W. Hänsch, Phys. Rev. A 22, 192 (1980)

503. T. Udem, J. Reichert, R. Holzwarth, T.W. Hänsch, Phys. Rev. Lett. 82, 3568 (1999)

504. P. Crivelli, C.L. Cesar, U. Gendotti, Can. J. Phys. 89, 29 (2011)

505. R.N. Fell, Phys. Rev. Lett. 68, 25 (1992)

506. A. Ore, Phys. Rev. 70, 90 (1946)

507. E.A. Hylleraas, A. Ore, Phys. Rev. 71, 493 (1947)

508. S. Bubin, M. Stanke, D. Kȩdziera, L. Adamowicz, Phys. Rev. A 75, 062504 (2007)

509. A.P. Mills Jr., Phys. Rev. Lett. 46, 717 (1981)

510. K. Varga, J. Usukura, Y. Suzuki, Phys. Rev. Lett. 80, $1876(1998)$

511. S. Bubin, O.V. Prezhdo, K. Varga, Phys. Rev. A 87, $054501(2013)$

512. K. Varga, Phys. Lett. A 378, 529 (2014)

513. A.M. Frolov, D.M. Wardlaw, Phys. Lett. A 372, 6721 (2008)

514. A.K. Bhatia, R.J. Drachman, Phys. Rev. A 28, 2523 (1983)

515. M. Born, R. Oppenheimer, Ann. Phys. 389, 457 (1927)

516. V.I. Korobov, Phys. Rev. A 61, 064503 (2000)

517. G.W.F. Drake, M. Grigorescu, J. Phys. B: At. Mol. Opt. Phys. 38, 3377 (2005)

518. R.A. Ferrell, Rev. Mod. Phys. 28, 308 (1956)

519. Y. Ho, Phys. Lett. A 144, 237 (1990)

520. M. Puchalski, A. Czarnecki, S.G. Karshenboim, Phys. Rev. Lett. 99, 203401 (2007)

521. E.A. Hylleraas, Phys. Rev. 71, 491 (1947)

522. Y.K. Ho, Phys. Rev. A 48, 4780 (1993)

523. A. Bhatia, R.J. Drachman, Nucl. Instrum. Methods Phys. Res. Sect. B: Beam Interact. Mater. At. 143, 95 (1998)

524. G.W.F. Drake, M.M. Cassar, R.A. Nistor, Phys. Rev. A 65, $054501(2002)$

525. A.M. Frolov, J. Phys. A: Math. Theor. 40, 6175 (2007)

526. N. Blinov, A. Czarnecki, Phys. Rev. A 85, 012522 (2012)

527. A.M. Frolov, Phys. Rev. A 60, 2834 (1999)

528. A. Igarashi, Few-Body Syst. 58, 2 (2016)

529. J. Botero, C.H. Greene, Phys. Rev. Lett. 56, 1366 (1986)

530. J.M. Rost, D. Wintgen, Phys. Rev. Lett. 69, 2499 (1992)

531. Y.K. Ho, A.K. Bhatia, Phys. Rev. A 47, 1497 (1993)

532. A.K. Bhatia, Y.K. Ho, Phys. Rev. A 48, 264 (1993)

533. Y. Zhou, C.D. Lin, Phys. Rev. Lett. 75, 2296 (1995)

534. I.A. Ivanov, Y.K. Ho, Phys. Rev. A 61, 032501 (2000)

535. J. Usukura, Y. Suzuki, Phys. Rev. A 66, 010502 (2002)

536. S. Kar, Y.K. Ho, Phys. Rev. A 86, 014501 (2012)

537. A.P. Mills Jr., Can. J. Phys. 91, 751 (2013)

538. A.P. Mills Jr., Phys. Rev. Lett. 50, 671 (1983)

539. A.P. Mills Jr., P.G. Friedman, D.M. Zuckerman, Decay rate and other properties of the positronium negative ion, in Annihilation in Gases and Galaxies, NASA Conference Publication (NASA, 1989), Vol. 3058, p. 213

540. D. Schwalm, F. Fleischer, M. Lestinsky, K. Degreif, G. Gwinner, V. Liechtenstein, F. Plenge, H. Scheit, in Proceedings of the XII International Workshop on Positron and Positronium Physics, Nucl. Instrum. Methods Phys. Res. Sect. B: Beam Interact. Mater. At. 221, 185 (2004) 
541. F. Fleischer, K. Degreif, G. Gwinner, M. Lestinsky, V. Liechtenstein, F. Plenge, D. Schwalm, Phys. Rev. Lett. 96, 063401 (2006)

542. H. Ceeh, C. Hugenschmidt, K. Schreckenbach, S.A. Gärtner, P.G. Thirolf, F. Fleischer, D. Schwalm, Phys. Rev. A 84, 062508 (2011)

543. Y. Nagashima, T. Sakai, New J. Phys. 8, 319 (2006)

544. Y. Nagashima, T. Hakodate, A. Miyamoto, K. Michishio, New J. Phys. 10, 123029 (2008)

545. H. Terabe, K. Michishio, T. Tachibana, Y. Nagashima, New J. Phys. 14, 015003 (2012)

546. D.W. Gidley, A.R. Köymen, T.W. Capehart, Phys. Rev. B 37, 2465 (1988)

547. A.R. Koymen, K.H. Lee, D. Mehl, A. Weiss, K.O. Jensen, Phys. Rev. Lett. 68, 2378 (1992)

548. N. Fazleev, J. Fry, K. Kuttler, A. Koymen, A. Weiss, in Proceedings of the Sixth International Workshop on SlowPositron Beam Techniques for Solids and Surfaces, Appl. Surf. Sci. 85, 26 (1995)

549. S.J. Buckman, C.W. Clark, Rev. Mod. Phys. 66, 539 (1994)

550. A. Igarashi, I. Shimamura, N. Toshima, New J. Phys. 2, $17(2000)$

551. S.J. Ward, J.W. Humberston, M.R.C. McDowell, J. Phys. B: At. Mol. Phys. 20, 127 (1987)

552. A.K. Bhatia, R.J. Drachman, Phys. Rev. A 32, 3745 (1985)

553. K. Wada et al., Eur. Phys. J. D 66, 37 (2012)

554. A.P. Mills, Phys. Rev. A 24, 3242 (1981)

555. U. Fano, Phys. Rev. 124, 1866 (1961)

556. M. Halka et al., Phys. Rev. A 46, 6942 (1992)

557. A.K. Bhatia, Y.K. Ho, Phys. Rev. A 42, 1119 (1990)

558. A. Igarashi, J. Phys. B: At. Mol. Opt. Phys. 45, 245201 (2012)

559. K. Michishio, R. Suzuki, K. Wada, I. Mochizuki, T. Hyodo, A. Yagishita, Y. Nagashima, Nucl. Instrum. Methods Phys. Res. Sect. A: Accel. Spectrom. Detect. Assoc. Equip. 785, 5 (2015)

560. B.L. Brown, Positron annihilation (World Scientific, Singapore, 1985), p. 328

561. M.H. Weber, S. Tang, S. Berko, B.L. Brown, K.F. Canter, K.G. Lynn, A.P. Mills, L.O. Roellig, A.J. Viescas, Phys. Rev. Lett. 61, 2542 (1988)

562. D.W. Gidley, R. Mayer, W.E. Frieze, K.G. Lynn, Phys. Rev. Lett. 58, 595 (1987)

563. A. Schüller, S. Wethekam, H. Winter, Phys. Rev. Lett. 98, 016103 (2007)

564. U.I. Uggerhøj, Phys. Rev. A 73, 052705 (2006)

565. E. Mátyus, M. Reiher, J. Chem. Phys. 137, 024104 (2012)

566. M.A. Lee, P. Vashishta, R.K. Kalia, Phys. Rev. Lett. 51, $2422(1983)$

567. D.B. Kinghorn, R.D. Poshusta, Phys. Rev. A 47, 3671 (1993)

568. D. Bressanini, M. Mella, G. Morosi, Phys. Rev. A 55, 200 (1997)

569. D.M. Schrader, Phys. Rev. Lett. 92, 043401 (2004)

570. M. Emami-Razavi, Phys. Rev. A 77, 042104 (2008)

571. I. Kylänpää, T.T. Rantala, Phys. Rev. A 80, 024504 (2009)

572. J. Mitroy, S. Bubin, W. Horiuchi, Y. Suzuki, L. Adamowicz, W. Cencek, K. Szalewicz, J. Komasa, D. Blume, K. Varga, Rev. Mod. Phys. 85, 693 (2013)
573. K.M. Daily, J. von Stecher, C.H. Greene, Phys. Rev. A 91, $012512(2015)$

574. Y.K. Ho, Phys. Rev. A 33, 3584 (1986)

575. P.M. Kozlowski, L. Adamowicz, Phys. Rev. A 48, 1903 1993

576. A.M. Frolov, S.I. Kryuchkov, V.H. Smith, Phys. Rev. A 51, 4514 (1995)

577. S. Bubin, L. Adamowicz, Phys. Rev. A 74, 052502 (2006)

578. D.H. Bailey, A.M. Frolov, Phys. Rev. A 72, 014501 (2005)

579. P.M. Kozlowski, L. Adamowicz, J. Phys. Chem. 100, 6266 (1996)

580. M. Puchalski, A. Czarnecki, Phys. Rev. Lett. 101, 183001 (2008)

581. A.J. Varandas, J. da Providência, M. Brajczewska, J.P. da Providência, Eur. Phys. J. D 69, 114 (2015)

582. J.R. Haynes, Phys. Rev. Lett. 17, 860 (1966)

583. W.F. Brinkman, T.M. Rice, B. Bell, Phys. Rev. B 8, 1570 (1973)

584. R.C. Miller, D.A. Kleinman, A.C. Gossard, O. Munteanu, Phys. Rev. B 25, 6545 (1982)

585. Y. Suzuki, J. Usukura, Nucl. Instrum. Methods Phys. Res. Sect. B: Beam Interact. Mater. At. 171, 67 (2000)

586. J. Usukura, K. Varga, Y. Suzuki, Phys. Rev. A 58, 1918 (1998)

587. D.B. Cassidy, S.H.M. Deng, A.P. Mills, Phys. Rev. A 76, $062511(2007)$

588. I.A. Ivanov, J. Mitroy, K. Varga, Phys. Rev. Lett. 87, $063201(2001)$

589. I.A. Ivanov, J. Mitroy, K. Varga, Phys. Rev. A 65, 022704 (2002)

590. T.D. Lee, C.N. Yang, Phys. Rev. 104, 254 (1956)

591. J.D. Jackson, S.B. Treiman, H.W. Wyld, Phys. Rev 106, $517(1957)$

592. P.W. Zitzewitz, J.C. Van House, A. Rich, D.W. Gidley, Phys. Rev. Lett. 43, 1281 (1979)

593. D.B. Cassidy, R.G. Greaves, V.E. Meligne, A.P. Mills Jr., Appl. Phys. Lett. 96, 101502 (2010)

594. A.P. Mills Jr. Nucl. Instrum. Methods Phys. Res. Sect. B: Beam Interact. Mater. At. 192, 107 (2002)

595. P.M. Platzman, A.P. Mills Jr., Phys. Rev. B 49, 454 (1994)

596. S. Chervenkov, X. Wu, J. Bayerl, A. Rohlfes, T. Gantner, M. Zeppenfeld, G. Rempe, Phys. Rev. Lett. 112, 013001 (2014)

597. G. Scholes, Atomic and molecular beam methods (Oxford University Press, New York, 1988), Vol. 1

598. S.E. Maxwell, N. Brahms, R. deCarvalho, D.R. Glenn, J.S. Helton, S.V. Nguyen, D. Patterson, J. Petricka, D. DeMille, J.M. Doyle, Phys. Rev. Lett. 95, 173201 (2005)

599. D.B. Cassidy, S.D. Hogan, Int. J. Mod. Phys.: Conf. Ser. 30, 1460259 (2014)

600. A. Gumberidze et al., Phys. Rev. Lett. 94, 223001 (2005)

601. J.N. Tan, S.M. Brewer, N.D. Guise, Phys. Scr. 2011, 014009 (2011)

602. D.Z. Kandula, C. Gohle, T.J. Pinkert, W. Ubachs, K.S.E. Eikema, Phys. Rev. A 84, 062512 (2011)

603. K. Pachucki, V.C.V. Patkóš, V.A. Yerokhin, Phys. Rev. A 95, 062510 (2017)

604. A.H. Hoang, P. Labelle, S.M. Zebarjad, Phys. Rev. Lett. 79, 3387 (1997)

605. W.E. Lamb, R.C. Retherford, Phys. Rev. 72, 241 (1947)

606. H. Lamm, Phys. Rev. A 96, 022515 (2017) 
607. M. Haghighat, S.M. Zebarjad, F. Loran, Phys. Rev. D 66, 016005 (2002)

608. R. Ley, K.D. Niebling, G. Werth, C. Hahn, H. Schneider, I. Tobehn, J. Phys. B: At. Mol. Opt. Phys. 23, 3437 (1990)

609. J.C. De Vries, Ph.D. thesis, Massachusetts Institute of Technology (2001)

610. F. Biraben, Eur. Phys. J. Special Topics 172, 109 (2009)

611. T.W. Hänsch, M.H. Nayfeh, S.A. Lee, S.M. Curry, I.S. Shahin, Phys. Rev. Lett. 32, 1336 (1974)

612. R. Pohl et al., Phys. Rev. Lett. 97, 193402 (2006)

613. K. Pachucki, Phys. Rev. A 53, 2092 (1996)

614. A. Beyer et al., Science 358, 79 (2017)

615. P.J. Mohr, D.B. Newell, B.N. Taylor, Rev. Mod. Phys. 88, 035009 (2016)

616. R. Pohl et al., Science 353, 669 (2016)

617. A. Antognini, F. Kottmann, F. Biraben, P. Indelicato, F. Nez, R. Pohl, Ann. Phys. 331, 127 (2013)

618. C.E. Carlson, Prog. Part. Nucl. Phys. 82, 59 (2015)

619. S.R. Lundeen, F.M. Pipkin, Phys. Rev. Lett. 46, 232 (1981)

620. E.W. Hagley, F.M. Pipkin, Phys. Rev. Lett. 72, 1172 (1994)

621. N.F. Ramsey, Rev. Mod. Phys. 62, 541 (1990)

622. F. Nez, M.D. Plimmer, S. Bourzeix, L. Julien, F. Biraben, R. Felder, Y. Millerioux, P. de Natale, Europhys. Lett. 24, 635 (1993)

623. B. de Beauvoir, F. Nez, L. Julien, B. Cagnac, F. Biraben, D. Touahri, L. Hilico, O. Acef, A. Clairon, J.J. Zondy, Phys. Rev. Lett. 78, 440 (1997)

624. C. Schwob, L. Jozefowski, B. de Beauvoir, L. Hilico, F. Nez, L. Julien, F. Biraben, O. Acef, J.J. Zondy, A. Clairon, Phys. Rev. Lett. 82, 4960 (1999)

625. V.A. Dzuba, V.V. Flambaum, G.F. Gribakin, W.A. King, Phys. Rev. A 52, 4541 (1995)

626. G.G. Ryzhikh, J. Mitroy, Phys. Rev. Lett. 79, 4124 (1997)

627. K. Strasburger, H. Chojnacki, J. Chem. Phys. 108, 3218 (1998)

628. J. Mitroy, M.W.J. Bromley, G.G. Ryzhikh, J. Phys. B: At. Mol. Opt. Phys. 35, R81 (2002)

629. V.A. Dzuba, V.V. Flambaum, G.F. Gribakin, C. Harabati, Phys. Rev. A 86, 032503 (2012)

630. X. Cheng, D. Babikov, D.M. Schrader, Phys. Rev. A 83, 032504 (2011)

631. C. Harabati, V.A. Dzuba, V.V. Flambaum, Phys. Rev. A 89, $022517(2014)$

632. V.A. Dzuba, V.V. Flambaum, G.F. Gribakin, Phys. Rev. Lett. 105, 203401 (2010)

633. C.M. Surko, J.R. Danielson, G.F. Gribakin, R.E. Continetti, New J. Phys. 14, 065004 (2012)

634. J.C. Greenbank, B.B. Argent, Trans. Faraday Soc. 61, 655 (1965)

635. B. Ghaffari, J.M. Gerton, W.I. McAlexander, K.E. Strecker, D.M. Homan, R.G. Hulet, Phys. Rev. A 60, 3878 (1999)

636. A. Osterwalder, EPJ Techniques and Instrumentation 2, $10(2015)$

637. S.D.S. Gordon, A. Osterwalder, Phys. Rev. Appl. 7, 044022 (2017)

638. H. Hotop, A. Niehaus, Z. Phys. 215, 395 (1968)

639. W.P. West, G.W. Foltz, F.B. Dunning, C.J. Latimer, R.F. Stebbings, Phys. Rev. Lett. 36, 854 (1976)
640. S.J. Brawley, S. Armitage, J. Beale, D.E. Leslie, A.I. Williams, G. Laricchia, Science 330, 789 (2010)

641. S.J. Brawley, A.I. Williams, M. Shipman, G. Laricchia, Phys. Rev. Lett. 105, 263401 (2010)

642. G.F. Chew, G.C. Wick, Phys. Rev. 85, 636 (1952)

643. I.I. Fabrikant, G.F. Gribakin, Phys. Rev. Lett. 112, $243201(2014)$

644. M. Shipman, S.J. Brawley, L. Sarkadi, G. Laricchia, Phys. Rev. A 95, 032704 (2017)

645. S. Willitsch, M.T. Bell, A.D. Gingell, S.R. Procter, T.P. Softley, Phys. Rev. Lett. 100, 043203 (2008)

646. B.C. Sawyer, B.K. Stuhl, M. Yeo, T.V. Tscherbul, M.T. Hummon, Y. Xia, J. Klos, D. Patterson, J.M. Doyle, J. Ye, Phys. Chem. Chem. Phys. 13, 19059 (2011)

647. M. Charlton, A.S. Kadyrov, I. Bray, Phys. Rev. A 94, 032701 (2016)

648. G.B. Andresen et al., Nature 468, 673 (2010)

649. G. Gabrielse et al. (ATRAP Collaboration), Phys. Rev. Lett. 108, 113002 (2012)

650. M. Doser et al., Class. Quantum Grav. 29, 184009 (2012)

651. P. Pérez et al., Hyperfine Interact. 233, 21 (2015)

652. W. Stoeffl, P. Asoka-Kumar, R. Howell, Appl. Surf. Sci. 149, 1 (1999)

653. G. Timp, R.E. Behringer, D.M. Tennant, J.E. Cunningham, M. Prentiss, K.K. Berggren, Phys. Rev. Lett. 69, 1636 (1992)

654. C.S. Allred, J. Reeves, C. Corder, H. Metcalf, J. Appl. Phys. 107, 033116 (2010)

655. Z. Liao, M. Al-Amri, T. Becker, W.P. Schleich, M.O. Scully, M.S. Zubairy, Phys. Rev. A 87, 023405 (2013)

656. E.A. Donley, N.R. Claussen, S.L. Cornish, J.L. Roberts, E.A. Cornell, C.E. Wieman, Nature 412, 295 (2001)

657. A.J. Leggett, Rev. Mod. Phys. 73, 307 (2001)

658. C. Pethick, H. Smith, Bose-Einstein condensation in dilute gases (Cambridge University Press, Cambridge, 2002)

659. A. Leggett, Quantum liquids: Bose condensation and Cooper pairing in condensed-matter systems, Oxford graduate texts in mathematics (OUP, Oxford, 2006)

660. H. Stolz, R. Schwartz, F. Kieseling, S. Som, M. Kaupsch, S. Sobkowiak, D. Semkat, N. Naka, T. Koch, H. Fehske, New J. Phys. 14, 105007 (2012)

661. H. Deng, H. Haug, Y. Yamamoto, Rev. Mod. Phys. 82, 1489 (2010)

662. M.H. Anderson, J.R. Ensher, M.R. Matthews, C.E. Wieman, E.A. Cornell, Science 269, 198 (1995)

663. K.B. Davis, M.O. Mewes, M.R. Andrews, N.J. van Druten, D.S. Durfee, D.M. Kurn, W. Ketterle, Phys. Rev. Lett. 75, 3969 (1995)

664. S. Chu, L. Hollberg, J.E. Bjorkholm, A. Cable, A. Ashkin, Phys. Rev. Lett. 55, 48 (1985)

665. A. Aspect, E. Arimondo, R. Kaiser, N. Vansteenkiste, C. Cohen-Tannoudji, Phys. Rev. Lett. 61, 826 (1988)

666. P.D. Lett, R.N. Watts, C.I. Westbrook, W.D. Phillips, P.L. Gould, H.J. Metcalf, Phys. Rev. Lett. 61, 169 (1988)

667. D.G. Fried, T.C. Killian, L. Willmann, D. Landhuis, S.C. Moss, D. Kleppner, T.J. Greytak, Phys. Rev. Lett. 81, 3811 (1998)

668. F. Pereira Dos Santos, J. Léonard, J. Wang, C.J. Barrelet, F. Perales, E. Rasel, C.S. Unnikrishnan, M. Leduc, C. Cohen-Tannoudji, Phys. Rev. Lett. 86, 3459 (2001)

669. S.C. Doret, C.B. Connolly, W. Ketterle, J.M. Doyle, Phys. Rev. Lett. 103, 103005 (2009) 
670. H. Iijima, T. Asonuma, T. Hirose, M. Irako, T. Kumita, M. Kajita, K. Matsuzawa, K. Wada, in Proceedings of the Int. Symp. on New Visions in Laser-Beam, Nucl. Instrum. Methods Phys. Res Sect. A: Accel. Spectrom. Detect. Assoc. Equip. 455, 104 (2000)

671. T. Kumita, T. Hirose, M. Irako, K. Kadoya, B. Matsumoto, K. Wada, N. Mondal, H. Yabu, K. Kobayashi, M. Kajita, Nucl. Instrum. Methods Phys. Res. Sect. B: Beam Interact. Mater. At. 192, 171 (2002)

672. P. Crivelli, D.A. Cooke, S. Friedreich, Int. J. Mod. Phys.: Conf. Ser. 30, 1460257 (2014)

673. J.L. Hall, C.J. Bordé, K. Uehara, Phys. Rev. Lett. 37, 1339 (1976)

674. D.S. Weiss, B.C. Young, S. Chu, Phys. Rev. Lett. 70, 2706 (1993)

675. C.W. Oates, G. Wilpers, L. Hollberg, Phys. Rev. A 71, $023404(2005)$

676. K. Shu, X. Fan, T. Yamazaki, T. Namba, S. Asai, K. Yoshioka, M. Kuwata-Gonokami, J. Phys. B: At. Mol. Opt. Phys. 49, 104001 (2016)

677. D. Kielpinski, Phys. Rev. A 73, 063407 (2006)

678. S. Wu, R.C. Brown, W.D. Phillips, J.V. Porto, Phys. Rev. Lett. 106, 213001 (2011)

679. A. Rich, J. Van House, D. Gidley, R. Conti, P. Zitzewitz, Appl. Phys. A 43, 275 (1987)

680. D. Abbott et al. (PEPPo Collaboration), Phys. Rev. Lett. 116, 214801 (2016)

681. D. Gerola, W.B. Waeber, M. Shi, S.J. Wang, Rev. Sci. Instrum. 66, 3819 (1995)

682. A.P. Mills, Appl. Phys. 23, 189 (1980)

683. W.E. Frieze, D.W. Gidley, K.G. Lynn, Phys. Rev. B 31 , $5628(1985)$

684. L.V. Jørgensen et al. (ATHENA Collaboration), Phys. Rev. Lett. 95, 025002 (2005)

685. D.W. Fitzakerley et al., J. Phys. B: At. Mol. Opt. Phys. 49, 064001 (2016)

686. C. Surko, R. Greaves, in Proceedings of the 7th International Conference on Positron and Positronium Chemistry, Radiat. Phys. Chem. 68, 419 (2003)

687. J.R. Danielson, N.C. Hurst, C.M. Surko, AIP Conf. Proc. 1521, 101 (2013)

688. C. Hugenschmidt, H. Ceeh, T. Gigl, F. Lippert, C. Piochacz, P. Pikart, M. Reiner, J. Weber, S. Zimnik, J. Phys.: Conf. Ser. 443, 012079 (2013)

689. T. Cowan, B. Beck, J. Hartley, R. Howell, R. Rohatgi, J. Fajans, R. Gopalan, Hyperfine Interact. 76, 135 (1993)

690. S. Grossmann, M. Holthaus, Z. Phys. B: Condens. Matter 97, 319 (1995)

691. K. Kirsten, D.J. Toms, Phys. Rev. E 59, 158 (1999)

692. H. Yabu, in Proceedings of the XII International Workshop on Positron and Positronium Physics, Nucl. Instrum. Methods Phys. Res. Sect. B: Beam Interact. Mater. At. 221, 144 (2004)

693. P. Froelich, S. Jonsell, V. Kharchenko, H.R. Sadeghpour, A. Dalgarno, J. Phys. B: At. Mol. Opt. Phys. 39, 3889 (2006)

694. Y.H. Wang, B.M. Anderson, C.W. Clark, Phys. Rev. A 89, 043624 (2014)

695. O. Morandi, P.A. Hervieux, G. Manfredi, Phys. Rev. A 89, 033609 (2014)

696. O. Morandi, P.A. Hervieux, G. Manfredi, J. Phys. B: At. Mol. Opt. Phys. 47, 155202 (2014)

697. O. Morandi, P.A. Hervieux, G. Manfredi, J. Phys. B: At. Mol. Opt. Phys. 49, 084002 (2016)
698. D.L. Griscom, Phys. Rev. B 64, 174201 (2001)

699. D. Cassidy, A. Mills, Nucl. Instrum. Methods Phys. Res. Sect. B: Beam Interact. Mater. At. 262, 59 (2007)

700. M. Eldrup, V. Shantarovich, O. Mogensen, Chem. Phys. 11, 129 (1975)

701. M. Kakimoto, T. Hyodo, T. Chiba, T. Akahane, T.B. Chang, J. Phys. B: At. Mol. Phys. 20, L107 (1987)

702. H. Saito, Y. Nagashima, T. Hyodo, T. Chang, Phys. Rev. B 52, R689 (1995)

703. H. Saito, T. Hyodo, Phys. Rev. B 60, 11070 (1999)

704. D.B. Cassidy, J.A. Golovchenko, The Bose-Einstein condensation of positronium in submicron cavities (Springer, Dordrecht, Netherlands, 2001), pp. 83-99

705. A.T. Lee, B. Cabrera, B.L. Dougherty, M.J. Penn, J.G. Pronko, S. Tamura, Phys. Rev. B 54, 3244 (1996)

706. J.M. Larkin, A.J.H. McGaughey, Phys. Rev. B 89, 144303 (2014)

707. M. Bertolotti, C. Sibilia, Appl. Phys. 19, 127 (1979)

708. A.O. Barut, N. Zanghi, Phys. Rev. Lett. 52, 20091984

709. C.M. Varma, Nature 267, 686 (1977)

710. A. Loeb, S. Eliezer, Laser Part. Beams 4, 577 (1986)

711. H.K. Avetissian, A.K. Avetissian, G.F. Mkrtchian, Phys. Rev. Lett. 113, 023904 (2014)

712. H.K. Avetissian, A.K. Avetissian, G.F. Mkrtchian, Phys. Rev. A 92, 023820 (2015)

713. V. Letokhov, Phys. Lett. A 49, 275 (1974)

714. G.C. Baldwin, J.C. Solem, V.I. Gol'danskii, Rev. Mod. Phys. 53, 687 (1981)

715. G.C. Baldwin, J.C. Solem, Rev. Mod. Phys. 69, 1085 (1997)

716. F. Vagizov, V. Antonov, Y.V. Radeonychev, R.N. Shakhmuratov, O. Kocharovskaya, Nature 508, 80 (2014)

717. A.S. Gevorkyan, K.B. Oganesyan, Y.V. Rostovtsev, G. Kurizki, Laser Phys. Lett. 12, 076002 (2015)

718. Z. Qi-Ren, Chin. Phys. B 24, 054208 (2015)

719. T.E.O. Ericson, A. Richter, Europhys. Lett. 11, 295 (1990)

720. S.G. Karshenboim, J. Phys. B: At. Mol. Opt. Phys. 49, $144001(2016)$

721. J. Scherk, Phys. Lett. B 88, 265 (1979)

722. M. Villata, Europhys. Lett. 94, 20001 (2011)

723. M. Villata, Ann. Phys. 527, 507 (2015)

724. D.S. Hajdukovic, Phys. Dark Universe 3, 34 (2014)

725. D.S. Hajdukovic, Int. J. Theor. Phys. 49, 1023 (2010)

726. D.S. Hajdukovic, Astrophys. Space Sci. 334, 219 (2011)

727. G. Chardin, J.M. Rax, Phys. Lett. B 282, 256 (1992)

728. D.S. Hajdukovic, Astrophys. Space Sci. 330, 1 (2010)

729. D.S. Hajdukovic, Astrophys. Space Sci. 334, 215 (2011)

730. M. Villata, Astrophys. Space Sci. 345, 1 (2013)

731. V.I. Sbitnev, Mod. Phys. Lett. A 30, 1550184 (2015)

732. A.R. Penner, Astrophys. Space Sci. 361, 124 (2016)

733. A. Einstein, Science 84, 506 (1936)

734. C.M. Will, Class. Quantum Grav. 32, 124001 (2015)

735. S. Schlamminger, K.Y. Choi, T.A. Wagner, J.H. Gundlach, E.G. Adelberger, Phys. Rev. Lett. 100, 041101 (2008)

736. L.I. Schiff, Phys. Rev. Lett. 1, 254 (1958)

737. R.V. Pound, G.A. Rebka, Phys. Rev. Lett. 4, 337 (1960)

738. G. Gabrielse, A. Khabbaz, D.S. Hall, C. Heimann, H. Kalinowsky, W. Jhe, Phys. Rev. Lett. 82, 3198 (1999)

739. M.H. Holzscheiter, Int. J. Mod. Phys.: Conf. Ser. 30, 1460260 (2014)

740. M.L. Good, Phys. Rev. 121, 311 (1961) 
741. D. Colladay, V.A. Kostelecký, Phys. Rev. D 58, 116002 (1998)

742. V.A. Kostelecký, J.D. Tasson, Phys. Rev. Lett. 102, 010402 (2009)

743. J.D. Tasson, Int. J. Mod. Phys.: Conf. Ser. 30, 1460273 (2014)

744. V.A. Kostelecký, A.J. Vargas, Phys. Rev. D 92, 056002 (2015)

745. E.G. Adelberger, B.R. Heckel, C.W. Stubbs, Y. Su, Phys. Rev. Lett. 66, 850 (1991)

746. T. Goldman, M.M. Nieto, M.H. Holzscheiter, T.W. Darling, M. Schauer, J. Schecker, Phys. Rev. Lett. 67, 1048 (1991)

747. M.J.T.F. Cabbolet, Astrophys. Space Sci. 337, 5 (2012)

748. T. Goldman, M.M. Nieto, Phys. Lett. B 112, 437 (1982)

749. F.C. Witteborn, W.M. Fairbank, Phys. Rev. Lett. 19, 1049 (1967)

750. A.J. Dessler, F.C. Michel, H.E. Rorschach, G.T. Trammell, Phys. Rev. 168, 737 (1968)

751. J.M. Lockhart, F.C. Witteborn, W.M. Fairbank, Phys. Rev. Lett. 38, 1220 (1977)

752. T.W. Darling, F. Rossi, G.I. Opat, G.F. Moorhead, Rev. Mod. Phys. 64, 237 (1992)

753. M.H. Holzscheiter, M. Charlton, M.M. Nieto, Phys. Rep. 402, 1 (2004)

754. G. Baur et al., Phys. Lett. B 368, 251 (1996)

755. G. Blanford, D.C. Christian, K. Gollwitzer, M. Mandelkern, C.T. Munger, J. Schultz, G. Zioulas, Phys. Rev. Lett. 80, 3037 (1998)

756. G. Gabrielse et al. (ATRAP Collaboration), Phys. Rev. Lett. 89, 213401 (2002)

757. Y. Enomoto et al., Phys. Rev. Lett. 105, 243401 (2010)

758. Y. Yamazaki, S. Ulmer, Ann. Phys. 525, 493 (2013)

759. The ALPHA Collaboration, A.E. Charman, Nat. Commun. 4, 1785 (2013)

760. G. Testera et al., Hyperfine Interact. 233, 13 (2015)

761. E. Hessels, D. Homan, M. Cavagnero, Phys. Rev. A 57, 1668 (1998)

762. P. Perez, Y. Sacquin, Class. Quantum Grav. 29, 184008 (2012)

763. A.P. Mills Jr., Hyperfine Interact. 44, 105 (1989)

764. T.J. Phillips, Hyperfine Interact. 109, 357 (1997)

765. M. Oberthaler, Nucl. Instrum. Methods Phys. Res. Sect. B: Beam Interact. Mater. At. 192, 129 (2002)

766. D. Delande, J.C. Gay, Europhys. Lett. 5, 303 (1988)

767. V.W. Hughes, D.W. McColm, K. Ziock, R. Prepost, Phys. Rev. Lett. 5, 63 (1960)

768. S. Chu, A.P. Mills Jr., A.G. Yodh, K. Nagamine, Y. Miyake, T. Kuga, Phys. Rev. Lett. 60, 101 (1988)

769. V. Meyer et al., Phys. Rev. Lett. 84, 1136 (2000)

770. A. Czarnecki, G.P. Lepage, W.J. Marciano, Phys. Rev. D 61, 073001 (2000)

771. A. Antognini et al., Phys. Rev. Lett. 108, 143401 (2012)

772. K.S. Khaw, A. Antognini, T. Prokscha, K. Kirch, L. Liszkay, Z. Salman, P. Crivelli, Phys. Rev. A 94, 022716 (2016)

773. K. Kirch, K.S. Khaw, Int. J. Mod. Phys.: Conf. Ser. 30, 1460258 (2014)

774. F. Hasselbach, Rep. Prog. Phys. 73, 016101 (2010)
775. K.P. Marzlin, Phys. Rev. A 88, 043621 (2013)

776. S. McGregor, R. Bach, H. Batelaan, New J. Phys. 13, 065018 (2011)

777. M.M. Dellweg, C. Müller, Phys. Rev. Lett. 118, 070403 (2017)

778. D.W. Keith, C.R. Ekstrom, Q.A. Turchette, D.E. Pritchard, Phys. Rev. Lett. 66, 2693 (1991)

779. A.D. Cronin, J. Schmiedmayer, D.E. Pritchard, Rev. Mod. Phys. 81, 1051 (2009)

780. M. Kasevich, S. Chu, Phys. Rev. Lett. 67, 181 (1991)

781. M.K. Oberthaler, S. Bernet, E.M. Rasel, J. Schmiedmayer, A. Zeilinger, Phys. Rev. A 54, 3165 (1996)

782. J.M. McGuirk, G.T. Foster, J.B. Fixler, M.J. Snadden, M.A. Kasevich, Phys. Rev. A 65, 033608 (2002)

783. R. Charrière, M. Cadoret, N. Zahzam, Y. Bidel, A. Bresson, Phys. Rev. A 85, 013639 (2012)

784. S. Dimopoulos, P.W. Graham, J.M. Hogan, M.A. Kasevich, S. Rajendran, Phys. Rev. D 78, 122002 (2008)

785. G.W. Biedermann, X. Wu, L. Deslauriers, S. Roy, C. Mahadeswaraswamy, M.A. Kasevich, Phys. Rev. A 91, 033629 (2015)

786. P. Hamilton, M. Jaffe, J.M. Brown, L. Maisenbacher, B. Estey, H. Müller, Phys. Rev. Lett. 114, 100405 (2015)

787. C. Bordé, Phys. Lett. A 140, 10 (1989)

788. P. Hamilton, A. Zhmoginov, F. Robicheaux, J. Fajans, J.S. Wurtele, H. Müller, Phys. Rev. Lett. 112, 121102 (2014)

789. J.F. Clauser, S. Li, Phys. Rev. A 49, R2213 (1994)

790. T. Brandt, T. Falke, W. Raith, Nucl. Instrum. Methods Phys. Res. Sect. B: Beam Interact. Mater. At. 149, 201 (1999)

791. O. Carnal, A. Faulstich, J. Mlynek, Appl. Phys. B 53, 88 (1991)

792. O. Carnal, J. Mlynek, Phys. Rev. Lett. 66, 2689 (1991)

793. M. Boustimi, J. Baudon, M. Ducloy, J. Reinhardt, F. Perales, C. Mainos, V. Bocvarski, J. Robert, Eur. Phys. J. D 17, $141(2001)$

794. C. Fabre, M. Gross, J.M. Raimond, S. Haroche, J. Phys. B: At. Mol. Phys. 16, L671 (1983)

795. A. Anderson, S. Haroche, E.A. Hinds, W. Jhe, D. Meschede, Phys. Rev. A 37, 3594 (1988)

796. P.J. Martin, B.G. Oldaker, A.H. Miklich, D.E. Pritchard, Phys. Rev. Lett. 60, 515 (1988)

797. E.M. Rasel, M.K. Oberthaler, H. Batelaan, J. Schmiedmayer, A. Zeilinger, Phys. Rev. Lett. 75, 2633 (1995)

798. S. Sala, F. Castelli, M. Giammarchi, S. Siccardi, S. Olivares, J. Phys. B: At. Mol. Opt. Phys. 48, 195002 (2015)

799. M. Arndt, A. Ekers, W. von Klitzing, H. Ulbricht, New J. Phys. 14, 125006 (2012)

800. K. Cassella, E. Copenhaver, B. Estey, Y. Feng, C. Lai, H. Müller, Phys. Rev. Lett. 118, 233201 (2017)

801. G.W. Biedermann, H.J. McGuinness, A.V. Rakholia, Y.Y. Jau, D.R. Wheeler, J.D. Sterk, G.R. Burns, Phys. Rev. Lett. 118, 163601 (2017)

802. H. Saito, T. Hyodo, in New directions in antimatter chemistry and Physics, edited by C.M. Surko, F.A. Gianturco (Springer, Dordrecht, Netherlands, 2001), pp. 101-114 